\title{
Stress Modulation on the San Andreas Fault by Interseismic Fault System Interactions
}

John P. Loveless

Harvard University, jloveles@smith.edu

Brendan J. Meade

Harvard University

Follow this and additional works at: https://scholarworks.smith.edu/geo_facpubs

Part of the Geology Commons

\section{Recommended Citation}

Loveless, John P. and Meade, Brendan J., "Stress Modulation on the San Andreas Fault by Interseismic Fault System Interactions" (2011). Geosciences: Faculty Publications, Smith College, Northampton, MA.

https://scholarworks.smith.edu/geo_facpubs/14 


\section{Stress modulation on the San Andreas fault by interseismic fault}

\section{2 system interactions}

3 John P. Loveless and Brendan J. Meade

4 Department of Earth and Planetary Sciences, Harvard University, Cambridge, Massachusetts

$5 \quad 02138, U S A$

6 ABSTRACT

7 During the interseismic phase of the earthquake cycle, between large earthquakes, stress

8 on faults evolves in response to elastic strain accumulation driven by tectonic plate motions.

9 Because earthquake cycle processes induce non-local stress changes, the interseismic stress

10 accumulation rate on one fault is influenced by the behavior of all nearby faults. Using a

11 geodetically constrained block model, we show that the total interseismic elastic strain field

12 generated by fault interactions within the southern California may increase stressing rates on the

13 Mojave and San Bernardino sections of the San Andreas fault within the Big Bend region by up

14 to $38 \%$ relative to estimates from isolated San Andreas models. Assuming steady fault system

15 behavior since the 1857 Fort Tejon earthquake, stress accumulated on these sections due only to

16 interaction with faults other than the San Andreas reaches $1 \mathrm{MPa}, \sim 3$ times larger than the

17 coseismic and postseismic stress changes induced by recent southern California earthquakes.

18 Stress increases along Big Bend sections coincide with the greatest earthquake frequency

19 inferred from a 1,500 year long paleoseismic record and may affect earthquake recurrence

20 intervals within geometrically complex fault systems including the sections of the San Andreas

21 fault closest to metropolitan Los Angeles.

\section{INTRODUCTION}


The San Andreas fault (SAF) in central-southern California accommodates as much as 35 $\mathrm{mm} / \mathrm{yr}$ (Sieh and Jahns, 1984) of the $53 \mathrm{~mm} / \mathrm{yr}$ of local relative motion between the Pacific and

25 North American plates (DeMets and Dixon, 1999). Historically, this motion has been expressed 26 seismically by the large $1812 \mathrm{M}_{\mathrm{W}}=7.5$ Wrightwood and $1857 \mathrm{M}_{\mathrm{W}}=7.9$ Fort Tejon earthquakes

27 (Sieh et al., 1989). Further evidence for large magnitude earthquakes has been documented

28 through paleoseismic studies revealing up to 14 earthquakes averaging of $3.2 \mathrm{~m}$ of slip per event 29 along the central-southern SAF since 550 CE (Weldon et al., 2004). The spatial distribution of 30 earthquake occurrence is not homogeneous over this time interval: seismic events have more 31 frequently ruptured the Big Bend of the SAF comprising the Mojave (MJ) and San Bernardino 32 sections (SB) within $50 \mathrm{~km}$ of metropolitan Los Angeles (Weldon et al., 2004; Biasi and 33 Weldon, 2009). During the past $\sim 150$ years of relative seismic quiescence, interseismic 34 earthquake cycle processes have continually modulated shear and Coulomb stresses on the SAF 35 while the seismically exposed population of greater Los Angeles has grown from less than 3610,000 to more than 10,000,000 (Stein and Hanks, 1998).

37 The total stress on a seismogenic fault surface results from the cumulative effects of 38 coseismic, postseismic, and interseismic earthquake cycle processes. Elastic models of a two39 hundred-year long historical earthquake catalog suggest abrupt coseismic Coulomb failure stress 40 perturbations up to $700 \mathrm{kPa}$ along the SAF (King et al., 1994; Freed et al., 2007). In the decade 41 following the $1992 \mathrm{M}_{\mathrm{W}}=7.3$ Landers and $1999 \mathrm{M}_{\mathrm{W}}=7.1$ Hector Mine earthquakes in the 42 eastern California shear zone (ECSZ), postseismic deformation, controlled by viscoelastic 43 relaxation processes in the lower crust and upper mantle (Smith and Sandwell, 2006), resulted in 44 a net $230-350 \mathrm{kPa}$ increase in CFS on $\mathrm{SB}$ and northern Indio section (IN) of the SAF and a 45 decrease on MJ of $100 \mathrm{kPa}$, reducing the likelihood of failure (Freed and Lin, 2002). Except 
46 following the largest earthquakes, such as the Fort Tejon event, the contribution of postseismic

47 deformation to the state of stress decays to apparently negligible levels within about a decade

48 (Freed et al., 2007).

49 In contrast to the short time scales associated with co- and postseismic stress changes,

50 interseismic stress accumulation is gradual yet characterizes the majority of each seismic cycle,

51 building cumulatively through the $10-100$ s of years between earthquakes and serving as the

52 primary mechanism driving future seismicity. On-fault stress accumulates through the

53 interseismic phase of the earthquake cycle when temporary frictional stability inhibits slip on the

54 seismogenic fault interface, above a locking depth defined by the brittle-ductile transition zone at

$55 \quad 15-25 \mathrm{~km}$ depth, causing a build-up of elastic strain within the upper crust (Fig. 1a; Savage and

56 Burford, 1973). In the seismogenic layer this effect is modeled using the slip deficit ("backslip")

57 method (Savage, 1983), where the slip deficit rate ranges from zero for a creeping fault to the

58 long-term fault slip rate for a locked fault. The interseismic stress accumulation rate on an

59 isolated fault is linearly proportional to its slip deficit rate (Fig. 1b; Okada, 1992). However,

60 stresses generated by slip deficit on one fault extend throughout the upper crust (e.g., Hetland

61 and Hager, 2006), decaying with distance as $\sim 1 / r$ for the near-field two-dimensional case.

62 Because of this non-local effect, the total stress accumulation on any fault segment is the sum of

63 the contributions from all active structures within the interacting fault network (Fig. 1b).

64 SAN ANDREAS FAULT STRESSING RATES

65 To calculate current stressing rates on the southern-central SAF, we use GPS

66 measurements of interseismic deformation (Fig. 2a) (McClusky et al., 2001; Shen et al., 2003;

67 Hammond and Thatcher, 2005; Williams et al., 2006; McCaffrey et al., 2007; Plate Boundary

68 Observatory network velocity field) and a three-dimensional spherical block model ${ }^{1}$ (Meade and 
69 Loveless, 2009) to constrain kinematically consistent slip rates (Weldon and Humphreys, 1986;

70 Minster and Jordan, 1987) on $\sim 60,000 \mathrm{~km}^{2}$ of fault area throughout the southern California fault

71 system (SCFS). Block models describe the interseismic GPS velocity field as the combined

72 effects of two processes, long-term microplate rotations and local elastic strain accumulation

73 effects (Savage and Burford, 1973; Savage, 1983; Matsu'ura et al., 1986). Using a fault system

74 geometry derived from the Southern California Earthquake Center Community Fault Model

75 (Plesch et al., 2007) (Table DR1) and 1822 GPS velocities (Table DR2), we simultaneously

76 estimate micro-plate rotations (yielding strike-slip rates and dip-slip (on dipping segments) or

77 opening/closing-slip (on vertical segments) rates on block-bounding faults), elastic strain

78 accumulation due to interseismic locking of faults, and homogeneous strain rates within crustal

79 micro-plates (McCaffrey, 2005). The block model fits the data with a mean residual velocity

80 magnitude of $1.67 \mathrm{~mm} / \mathrm{yr}$ and simultaneously satisfies far-field Pacific-North America plate

81 motion constraints (DeMets and Dixon, 1999).

82

Model results show that slip on the Carrizo section (CZ) of the SAF currently accounts

83 for up to $60 \%$ of the $53 \mathrm{~mm} / \mathrm{yr}$ of relative plate motion (DeMets and Dixon, 1999). However, the

84 anastomosing geometry of the SCFS partitions slip across multiple faults through the Big Bend

85 region (Fig. 2b) and, as a consequence, SAF strike-slip rates vary significantly along strike: 31.2

$86 \pm 0.2 \mathrm{~mm} / \mathrm{yr}$ on $\mathrm{CZ}, 16.3 \pm 0.8 \mathrm{~mm} / \mathrm{yr}$ on $\mathrm{MJ}, 10.2 \pm 0.3 \mathrm{~mm} / \mathrm{yr}$ on $\mathrm{SB}, 25.4 \pm 0.2 \mathrm{~mm} / \mathrm{yr}$ on $\mathrm{IN}$

87 and $39.2 \pm 0.4 \mathrm{~mm} / \mathrm{yr}$ on the Imperial section (IM) (Fig. 2b, Table DR1). The remaining 16\%-

$8874 \%(\sim 10-40 \mathrm{~mm} / \mathrm{yr})$ of relative plate motion is distributed among other active faults, most

89 significantly the San Jacinto, Elsinore, and Hosgri faults and the ECSZ (McClusky et al., 2001)

90 (Fig. 2b). The along-strike variation in SAF strike-slip rate, reaching a minimum along SB, is

91 consistent with late Pleistocene estimates (McGill et al., 2010). 
These fault slip rates inferred from the geodetically constrained block model, interpreted

93 as slip deficit rates, provide the basis for determining present-day interseismic stress

94 accumulation rates on the SAF (Fig. 1, 3, DR1-DR3). We analytically (Okada, 1992) calculate

95 shear stress rates every $\sim 10 \mathrm{~km}$ along strike at the centroid of each SAF segment, with the

96 centroid depth defined as half the depth to which the segment is inferred to be interseismically

97 locked. The self-stress rate, $\tau_{\mathrm{SAF}}$, results from slip deficit only on SAF segments and total stress

98 rate on the fault, $\tau_{\mathrm{TOT}}$, is due to slip deficit on all fault segments of the SCFS. Differential stress

99 rates, $\Delta \tau=\tau_{\mathrm{TOT}}-\tau_{\mathrm{SAF}}$, and the normalized percent difference, $\Delta \tau^{\prime}=\Delta \tau / \tau_{\mathrm{SAF}} \times 100$, represent the

100 modulation of SAF stress rate due to all faults other than the SAF.

101 Self shear stress rates are greatest $(60-80 \mathrm{kPa} / \mathrm{yr})$ along the southern IN and northern IM

102 and least (11-14 kPa/yr) along MJ and SB (Fig. 3a) where fault slip deficit rates are lowest. Slip

103 deficit rates are highest along CZ and IM (31.5 and $38 \mathrm{~mm} / \mathrm{yr}$, respectively) (Fig. 2b), but $\tau_{\mathrm{SAF}}$

104 are moderate $(\sim 18-32 \mathrm{kPa} / \mathrm{yr})$ due to greater locking depths along CZ (20-25 km) and IM (15

$105 \mathrm{~km})$ than along IN (5.0-7.5 km) (Table DR1). Total stress rates, $\tau_{\mathrm{TOT}}$, show a similar spatial

106 distribution to $\tau_{\mathrm{SAF}}$, but are significantly higher ( 14-17 kPa/yr) along MJ and SB (Fig. 2b).

107 Stress rate modulation, $\Delta \tau$, , shows peaks at fault junctions, which result from

108 singularities at fault triple junctions that are incompletely cancelled by adjacent segments in the

109 fault geometry subset considered in calculating $\tau_{\mathrm{SAF}}$. These artifacts persist for only $\sim 10 \mathrm{~km}$, and

110 we focus on values away from junctions, which reach $+38 \%$ along MJ and SB (Fig. 3b). Stress

111 increases (positive $\Delta \tau^{\prime}$ ) results from the cumulative effect of the interseismic stress fields

112 generated by the nearby White Wolf, Garlock, San Gabriel, North Frontal, Eureka Peak, and San

113 Jacinto faults, as well as the ECSZ. Assuming a $45^{\circ}$ north-dipping San Gorgonio Pass fault

114 segment, $\Delta \tau^{\prime}$ along SB is decreased by an average of $8.7 \%$ relative to the vertically dipping 
115 reference model, primarily due to three negative $\Delta \tau^{\prime}$ dipping segments; the overall SB stress

116 increases are similar. Except at fault junctions, $\Delta \tau^{\prime}$ is negligible along $\mathrm{CZ}$, IN, and IM where the

117 SAF is relatively isolated from neighboring faults.

\section{DISCUSSION}

119 A consequence of the increased shear stress rates is that interseismic fault system

120 interactions may reduce the time required to accumulate the stresses necessary for seismic failure

121 on MJ and SB relative to the time predicted by $\tau_{\mathrm{SAF}}$ alone. Annual $\Delta \tau$ along SB (mean 2.8

$122 \mathrm{kPa} / \mathrm{yr}$, maximum $6.8 \mathrm{kPa} / \mathrm{yr}$ ) is $\sim 2$ orders of magnitude smaller than the co- and post-seismic

123 stress changes induced by the recent Landers and Hector Mine ECSZ earthquakes (Freed and

124 Lin, 2002). However, the cumulative effect of interseismic shear stress modulation reaches a

125 maximum of $1 \mathrm{MPa}$ when integrated over the $\sim 150$ years since the 1857 Fort Tejon earthquake.

126 This value is $\sim 3$ times the maximum ( $\sim 300 \mathrm{kPa})$ shear stress induced by the Landers earthquake

127 on SB (King et al., 1994). Thus, in terms of stress perturbations that may trigger earthquakes and

128 control long-term seismicity patterns, interseismic stress modulation is of similar magnitude to

129 co- and post-seismic sources along SB and, more importantly, continues to influence this section

130 long after the earthquake-related stress changes have decayed to negligible levels, though

131 ongoing postseismic deformation occurring at a rate below the current detection limits of GPS

132 (Freed et al., 2007) may be considered a part of the nominally interseismic period. Coulomb

133 failure stress (CFS $=\tau-\mu \sigma_{\mathrm{n}}$, where $\mu$ is the effective coefficient of friction and $\sigma_{\mathrm{n}}$ is normal

134 stress) on MJ was reduced up to $50 \mathrm{kPa}$ coseismically due to the Landers earthquake (King et al.,

135 1994; Freed and Lin, 2002) and up to $100 \mathrm{kPa}$ by postseismic deformation following the Landers

136 and Hector Mine events (Freed and Lin, 2002). In contrast, interseismic fault system interactions

137 have induced a positive CFS change of up to $540 \mathrm{kPa}$ during the post-Fort Tejon epoch 
$138\left(\mathrm{CFS}_{\mathrm{TOT}}-\mathrm{CFS}_{\mathrm{SAF}} \approx 3.6 \mathrm{kPa} / \mathrm{yr}\right.$ maximum; $1.8 \mathrm{kPa} / \mathrm{yr}$ mean $)$. This outweighs the negative changes

139 caused by recent ECSZ earthquakes by 440-490 kPa and suggests that interseismic fault system

140 interactions over the past 150 years have been the largest magnitude off-SAF source of stress

141 with the potential to reduce earthquake recurrence intervals.

142 Assuming uniform fault strength along strike and homogeneous coseismic stress drops,

143 estimated recurrence intervals of large earthquakes on the entire SAF based on $\tau_{\mathrm{SAF}}$ or $\tau_{\mathrm{TOT}}$

144 would suggest that earthquakes occur more frequently on the high stress rate CZ, IN, and IM

145 than along MJ and SB. However, this simple idealization is inconsistent with paleoseismic data,

146 which suggest that Big Bend sections have ruptured in at least as many earthquakes as CZ (Grant

147 Ludwig et al., 2010) and more than IN and IM (Weldon et al., 2004; Biasi and Weldon, 2009).

148 Based on data compiled at paleoseismic sites along the SAF between CZ and IN, admissible

149 rupture models (Weldon et al., 2004; Biasi and Weldon, 2009) support the occurrence of 12-26

150 earthquakes in the past 1500 yr. For these models, we count the number of inferred events that

151 involved each $\sim 10 \mathrm{~km}$-long segment of the SAF used in our stress rate calculations (Fig. $3 \mathrm{~b}$ ).

152 Though the models vary in number of events and rupture extents due to uncertainties in

153 stratigraphic offset and age measurements and correlation of data from site to site (Biasi and

154 Weldon, 2009), those that have been published (Weldon et al., 2004; Biasi and Weldon, 2009)

155 show a greater number of inferred events rupturing MJ and SB than elsewhere along strike,

156 where $\Delta \tau^{\prime}$ is generally positive (Fig. 3b). Interseismic stress modulation may, however, be

157 modified by subsequent co- and postseismic effects, such as the decrease in failure stress induced

158 on MJ by ECSZ earthquakes (King et al., 1994). Therefore, any relationship between $\Delta \tau^{\prime}$ and

159 earthquake recurrence interval may only be relevant during a single interseismic phase. 
160 Regardless, SAF earthquake recurrence intervals estimated from $\tau_{\mathrm{SAF}}$, assuming uniform fault

161 strength and coseismic stress drop, may be overestimated.

\section{ACKNOWLEDGMENTS}

163

164

165

166

167

168

169

170

171

172

173

174

175

176

177

178

179

180

Figures $2 \& 3$ created with the Generic Mapping Tools (Wessel and Smith, 1998). We thank James Dolan and an anonymous referee for helpful reviews and Michele Cooke for reviewing a previous version. Figures 2 and 3 were prepared using the Generic Mapping Tools (Wessel and Smith, 1998). This work is supported by funding from Harvard University and the Southern California Earthquake Center.

\section{REFERENCES CITED}

Biasi, G.P., and Weldon, R.J., 2009, San Andreas fault rupture scenarios from multiple paleoseismic records: Stringing pearls: Bulletin of the Seismological Society of America, v. 99 , p. 471-498, doi:10.1785/0120080287.

DeMets, C., and Dixon, T.H., 1999, New kinematic models for Pacific-North America motion from 3 Ma to present, I: Evidence for steady motion and biases in the NUVEL-1A model: Geophysical Research Letters, v. 26, p. 1921-1924, doi:10.1029/1999GL900405.

Freed, A.M., and Lin, J., 2002, Accelerated stress buildup on the southern San Andreas fault and surrounding regions caused by Mojave Desert earthquakes: Geology, v. 30, p. 571-574, doi:10.1130/0091-7613(2002)030<0571:ASBOTS>2.0.CO;2.

Freed, A.M., Ali, S.T., and Bürgmann, R., 2007, Evolution of stress in Southern California for the past 200 years from coseismic, postseismic and interseismic stress changes: Geophysical Journal International, v. 169, p. 1164-1179, doi:10.1111/j.1365-246X.2007.03391.x. 
181 Grant Ludwig, L., Akçiz, S.O., Noriega, G.R., Zielke, O., and Arrowsmith, J.R., 2010, Climate-

182 modulated channel incision and rupture history of the San Andreas fault in the Carrizo Plain:

183 Science, v. 327, p. 1117-1119, doi:10.1126/science.1182837.

184 Hammond, W.C., and Thatcher, W., 2005, Northwest Basin and Range tectonic deformation 185 observed with the Global Positioning System, 1999-2003: Journal of Geophysical Research, 186 v. 110, p. B10405, doi:10.1029/2005JB003678.

187 Hetland, E.A., and Hager, B.H., 2006, Interseismic strain accumulation: Spin-up, cycle 188 invariance, and irregular rupture sequences: Geochemistry Geophysics Geosystems, v. 7, 189 p. Q05004, doi:10.1029/2005GC001087.

190 King, G.C.P., Stein, R.S., and Lin, J., 1994, Static stress changes and the triggering of 191 earthquakes: Bulletin of the Seismological Society of America, v. 84, p. 935-953.

192 Matsu'ura, M., Jackson, D. D., and Cheng A., 1986, Dislocation model for aseismic crustal 193 deformation at Hollister, California: Journal of Geophysical Research, v. 91, no. B12, p. $194 \quad 2661-2674$.

195 McCaffrey, R., 2005, Block kinematics of the Pacific-North America plate boundary in the 196 southwestern United States from inversion of GPS, seismological, and geologic data: 197 Journal of Geophysical Research, v. 110, p. B07401, doi:10.1029/2004JB003307.

198 McCaffrey, R., Qamar, A.I., King, R.W., Wells, R., Khazaradze, G., Williams, C.A., Stevens, 199 C.W., Vollick, J.J., and Zwick, P.C., 2007, Fault locking, block rotation and crustal 200 deformation in the Pacific Northwest: Geophysical Journal International, v. 169, p. 1315$201 \quad$ 1340, doi:10.1111/j.1365-246X.2007.03371.x.

202 McClusky, S.C., Bjornstad, S.C., Hager, B.H., King, R.W., Meade, B.J., Miller, M.M., 203 Monastero, F.C., and Souter, B.J., 2001, Present day kinematics of the Eastern California 
Shear Zone from a geodetically constrained block model: Geophysical Research Letters, v. 28, p. 3369-3372, doi:10.1029/2001GL013091.

McGill, S., Weldon, R., and Owen, L., 2010, Latest Pleistocene slip rates along the San Bernardino Strand of the San Andreas fault: Geological Society of America Abstracts with Programs, v. 42, no. 4, p. 69.

Meade, B.J., and Loveless, J.P., 2009, Block modeling with connected fault network geometries and a linear elastic coupling estimator in spherical coordinates: Bulletin of the Seismological Society of America, v. 99, p. 3124-3139, doi:10.1785/0120090088.

Minster, J.B., and Jordan, T.H., 1987, Vector constraints on western United-States deformation from space geodesy, neotectonics, and plate motions: Journal of Geophysical Research,

Okada, Y., 1992, Internal deformation due to shear and tensile faults in a half-space: Bulletin of the Seismological Society of America, v. 82, p. 1018-1040.

217 Plate Boundary Observatory network velocity field: http://pboweb.unavco.org.

218 Plesch, A., Shaw, J.H., Benson, C., Bryant, W.A., Carena, S., Cooke, M., Dolan, J., Fuis, G., 219 Gath, E., Grant, L., et al., 2007, Community Fault Model (CFM) for Southern California: 220 Bulletin of the Seismological Society of America, v. 97, p. 1793-1802, 221 doi:10.1785/0120050211.

222 Savage, J.C., 1983, A dislocation model of strain accumulation and release at a subduction zone: 223 Journal of Geophysical Research, v. 88, p. 4984-4996, doi:10.1029/JB088iB06p04984. 224 Savage, J.C., and Burford, R.O., 1973, Geodetic determination of relative plate motion in central 225 California: Journal of Geophysical Research, v. 78, p. 832-845, 226 doi:10.1029/JB078i005p00832. 
Shen, Z.K., Agnew, D.C., King, R.W., Dong, D., Herring, T.A., Wang, M., Johnson, H., Anderson, G., Nikolaidis, R., van Domselaar, M., Hudnut, K.W., and Jackson, D.D., 2003, SCEC Crustal Motion Map, Version 3.0: http://epicenter.usc.edu/cmm3.

Sieh, K., Stuiver, M., and Brillinger, D., 1989, A more precise chronology of earthquakes produced by the San Andreas fault in southern California: Journal of Geophysical Research, v. 94, p. 603-623, doi:10.1029/JB094iB01p00603.

Sieh, K.E., and Jahns, R.H., 1984, Holocene Activity of the San-Andreas Fault at WallaceCreek, California: Geological Society of America Bulletin, v. 95, p. 883-896, doi:10.1130/0016-7606(1984)95<883:HAOTSA >2.0.CO;2.

Smith, B.R., and Sandwell, D.T., 2006, A model of the earthquake cycle along the San Andreas Fault System for the past 1000 years: Journal of Geophysical Research, v. 111, p. B01405, doi:10.1029/2005JB003703.

Stein, R.S., and Hanks, T.C., 1998, M >= 6 earthquakes in southern California during the twentieth century: No evidence for a seismicity or moment deficit: Bulletin of the Seismological Society of America, v. 88, p. 635-652.

Weldon, R., and Humphreys, E., 1986, A kinematic model of Southern California: Tectonics, v. 5, p. 33-48, doi:10.1029/TC005i001p00033.

Weldon, R., Scharer, K., Fumal, T., and Biasi, G., 2004, Wrightwood and the earthquake cycle: What a long recurrence record tells us about how faults work: GSA Today, v. 14, no. 9, p. 4-10, doi:10.1130/1052-5173(2004)014<4:WATECW>2.0.CO;2.

Wesnousky, S.G., 2006, Predicting the endpoints of earthquake ruptures: Nature, v. 444, p. 358360, doi:10.1038/nature05275. 
249 Wessel, P., and Smith, W.H.F., 1998, New, improved version of Generic Mapping Tools: Eos,

250 Transactions, American Geophysical Union, v. 79, p. 579, doi:10.1029/98EO00426.

251 Williams, T.B., Kelsey, H.M., and Freymueller, J.T., 2006, GPS-derived strain in northwestern

252 California: Termination of the San Andreas fault system and convergence of the Sierra

253 Nevada-Great Valley block contribute to southern Cascadia forearc contraction:

254 Tectonophysics, v. 413, p. 171-184, doi:10.1016/j.tecto.2005.10.047.

\section{FIGURE CAPTIONS}

256 Figure 1. A) Interseismic fault-parallel surface velocities, $v$, due to slip deficit on two faults

257 locked to depth $d$ and spaced $W=2 d$. The slip deficit rate is $v_{0}$ on fault 1 (blue) and $v_{0} / 2$ on fault

2582 (red). Total velocity due to slip deficit on both faults is shown in black. B) Interseismic surface

259 shear stress accumulation rates, $\tau$. The difference between total stress accumulation rate (black

260 line) and the self-stressing rate on fault 2 (red line), $\Delta \tau$, represents modulation of stress on one

261 fault due to slip deficit on the other. Inset shows decay of $\Delta \tau$ with fault separation, $W / d$.

262 Figure 2. Block model constraints and results. A) Interseismic velocity field (McClusky et al.,

263 2001; Shen et al., 2003; Hammond and Thatcher, 2005; Williams et al., 2006; McCaffrey et al.,

264 2007; Plate Boundary Observatory network velocity field) relative to stable North America.

265 Arrow length is uniform; speed is denoted by color. B) Estimated strike-slip rates on block

266 bounding faults from our reference block model given by colored lines (right-lateral is positive).

267 Gray lines show block geometry, which is constructed by connecting faults with parameters

268 specified by the SCEC CFM-R (Plesch et al., 2007) (black lines). Selected faults are labeled with

269 average slip rate, rounded to nearest mm/yr; G: Garlock, WW: White Wolf. See Table DR1 for

$270 \quad$ full listing of slip rates. 
271 Figure 3. Interseismic shear stressing rates calculated analytically (Okada, 1992) using the slip

272 deficit rates from our kinematically consistent block model. Two quantities are plotted in each

273 panel, one to the left (southwest) and one to the right (northeast) of the SAF trace, as labeled at the

274 bottom of the panel. A) Self-shear stress rate due to slip deficit on San Andreas fault (SAF)

275 segments alone, $\tau_{\mathrm{SAF}}$, and total shear stress rate due to slip deficit on all faults, $\tau_{\mathrm{TOT}}$. Fault

276 junctions are labeled in white; WW: White Wolf, GL: Garlock, SG: San Gabriel, SJ: San Jacinto,

277 NF: North Frontal, EP: Eureka Peak, EL: Elsinore. Junctions bound labeled sections of the SAF

278 (black italics); SB: San Bernardino. B) Percent difference between total and self-stress,

279 normalized by self-stress, $\Delta \tau^{\prime}$ (“\% diff.”), and mean number of paleoseismic earthquakes on each

280 model segment (“\# EQ”), from the rupture models of Biasi and Weldon (2009).

$281{ }^{1}$ GSA Data Repository item 2011xxx, text, Tables DR1-DR2, and Figures DR1-DR3, is

282 available online at www.geosociety.org/pubs/ft2011.htm, or on request from

283 editing@geosociety.org or Documents Secretary, GSA, P.O. Box 9140, Boulder, CO 80301, 284 USA. 


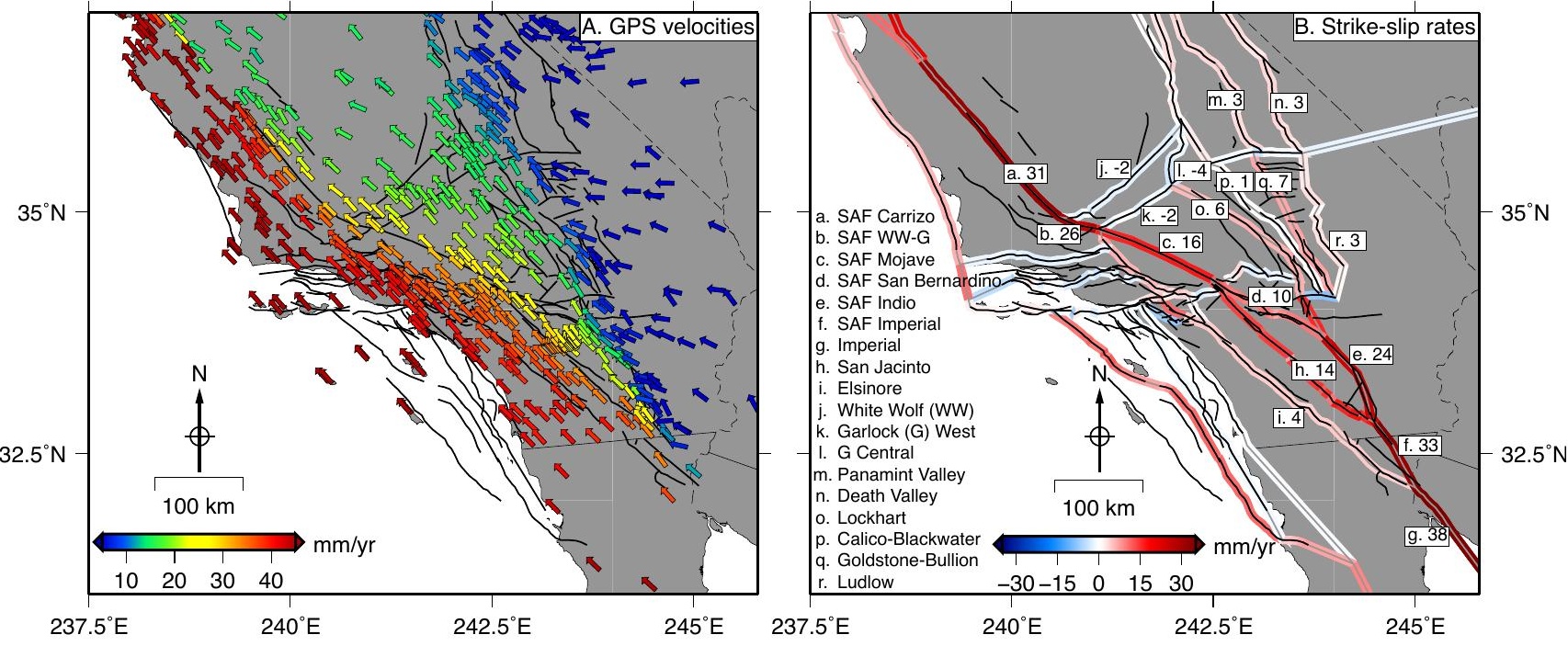




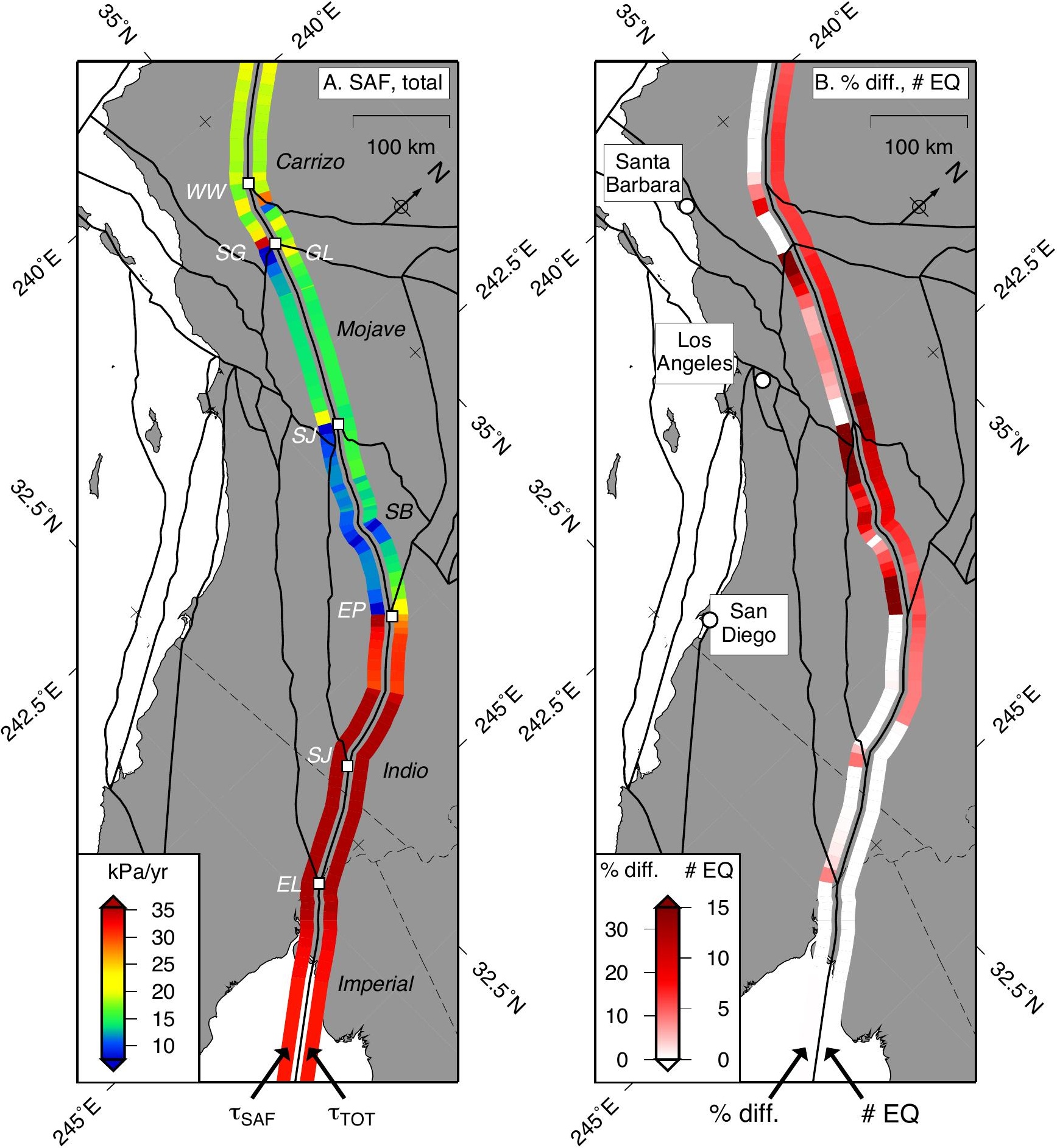




\section{Stress amplification on the San Andreas fault due to interseismic fault system interactions: Supplementary Information}

John P. Loveless and Brendan J. Meade

Department of Earth and Planetary Sciences

Harvard University

20 Oxford Street, Cambridge, MA 02138 USA

loveless@eps.harvard.edu

\section{WESTERN UNITED STATES BLOCK MODEL AND FAULT SLIP RATES}

We construct a three-dimensional, spherical block model geometry of interconnected faults throughout the entire western United States, integrating the southern California fault system (SCFS) with San Francisco Bay Area faults, Basin and Range structures, and the Cascadia subduction zone in the Pacific Northwest. In southern California, we use the Southern California Earthquake Center's Community Fault Model (CFM) (Plesch et al., 2007) and United States Geological Survey Quaternary fault map as the bases for the block geometry, using the CFM-specified dips for most fault segments. The model is constrained by a nominally interseismic velocity field from 6 published networks (McClusky et al., 2001; Shen et al., 2003; Hammond and Thatcher, 2005; McCaffrey, 2005; Williams et al., 2006; Plate Boundary Observatory network velocity field, 2008), combined into a common reference frame by minimizing velocity misfit between collocated stations in the fields, using a 6-parameter (rotation plus translation) transformation.

We use these data and the theory presented by Meade and Loveless (2009) to estimate a suite of kinematically consistent slip rates on fault segments bounding crustal blocks. The basis for block theory is the earthquake cycle, in which net "long-term" displacement between adjacent blocks at the end of one complete cycle is given as the sum of interseismic and coseismic deformation. The GPS data used to constrain the model measures the nominally interseismic velocity field, $\mathbf{v}_{\mathrm{I}}$, which is given as the difference between the step-like, long-term block deformation, $\mathbf{v}_{\mathrm{B}}$, and the velocities related to coseismic elastic deformation, $\mathbf{v}_{\mathrm{E}}: \mathbf{v}_{\mathrm{I}}=\mathbf{v}_{\mathrm{B}}-\mathbf{v}_{\mathrm{E}}$. We estimate an Euler pole of rotation for each crustal block in the model and project the relative rotational motion of two adjacent blocks onto the fault segments that bound them to give a set of block-motion slip rates that inherently satisfy path integral constraints. Assuming that the fault segments are fully locked from the surface to an assumed locking depth, the elastic strain contribution to the velocity field is proportional to the kinematically consistent fault slip rate. We can therefore interpret the blockmotion slip rates as slip deficit rates, giving the annual accrual of slip that will be released in the next earthquake.

The constraining GPS data, $\mathbf{v}_{\mathrm{I}}$, is related to the block rotation vectors, $\Omega$, slip rates on partially coupled dislocation sources, $\mathbf{p}$, and internal strain rate tensors, $\dot{\epsilon}$, through the equation $\mathbf{v}_{\mathrm{I}}=\mathbf{G}[\Omega \mathbf{p} \dot{\epsilon}]^{\top}$, where $\mathbf{G}$ is a combined Jacobian, and we estimate the model parameters using a linear weighted least-squares inversion. The estimated relative block rotations are projected onto the three dimensional fault system geometry, providing a kinematically consistent set of faultparallel and fault-normal slip rates that inherently satisfy path integral constraints. We estimate two components of fault slip rates on each segment: strike-slip rates are estimated for all faults, and the 
fault-normal slip rate is given as dip-slip on dipping faults and tensile (opening or closing sense) slip on vertical faults. The contribution to the velocity field from elastic strain accumulation about locked faults scales linearly with these slip rates and is incorporated into $\mathbf{G}$ using elastic dislocation Green's functions (Okada, 1992). Partially coupled fault surfaces — in this case, the Cascadia subduction zone and Parkfield segment of the San Andreas fault (SAF) - are parametrized by triangular dislocation elements (Meade, 2007). The total contribution to the interseismic velocity field from elastic strain accumulation is subtracted from the velocity field due to block rotation alone, reflecting the We present geometric parameters and estimated fault slip rates for southern California faults in Table DR1 and the combined interseismic GPS velocity field in Table DR2.

\section{STRESS ACCUMULATION RATES}

To calculate stress accumulation rates on SAF, we use our catalog of derived slip rates (Fig. 1B, Table DR1), analytical expressions (Okada, 1992) for strain due to dislocations embedded in a homogenous elastic half-space, and constitutive relations for converting strain rates to stress rates.

We formulate the expressions as follows. For each SAF segment centroid, we calculate the partial derivatives, $\mathbf{G}_{\mathrm{TOT}}$, giving the strain rate tensor components resulting from unit dislocations on all segments in the SCFS. To convert strain rate, $\epsilon$, to stress rate, $\sigma$, we assume a linear Hookean rheology, $\sigma_{i j}=\lambda \epsilon_{k k} \delta_{i j}+2 \mu \epsilon_{i j}$, where $\lambda$ and $\mu$ are the Lamé parameters (assumed to be $\lambda=\mu=$ $3 \times 10^{10} \mathrm{~Pa}$ ) and $\delta_{i j}$ is the Kronecker delta, summed over $i, j=1 \ldots 3$. This can be expressed as a linear operator,

$$
\mathbf{T}=\left[\begin{array}{cccccc}
\lambda+2 \mu & \lambda & \lambda & 0 & 0 & 0 \\
\lambda & \lambda+2 \mu & \lambda & 0 & 0 & 0 \\
\lambda & \lambda & \lambda+2 \mu & 0 & 0 & 0 \\
0 & 0 & 0 & 2 \mu & 0 & 0 \\
0 & 0 & 0 & 0 & 2 \mu & 0 \\
0 & 0 & 0 & 0 & 0 & 2 \mu
\end{array}\right]
$$

multiplying the strain rate tensor components, $\boldsymbol{\epsilon}=\left[\epsilon_{11}, \epsilon_{22}, \epsilon_{33}, \epsilon_{12}, \epsilon_{13}, \epsilon_{23}\right]^{\top}$, so that stress rate can be calculated as $\boldsymbol{\sigma}_{\mathrm{TOT}}=\underline{\mathbf{T}} \mathbf{G}_{\mathrm{TOT}} u_{\mathrm{TOT}}=\mathbf{H}_{\mathrm{TOT}} u_{\mathrm{TOT}}$, where $\underline{\mathbf{T}}$ is a $6 n_{\mathrm{SAF}} \times 6 n_{\mathrm{SAF}}$ block diagonal matrix with entries $\mathbf{T}$, and $\mathbf{H}=\underline{\mathbf{T}} \mathbf{G}$ is a combined linear operator, and $u_{\mathrm{TOT}}$ is the vector of slip rates. $\mathrm{G}_{\mathrm{TOT}}$ is a $6 n_{\mathrm{SAF}} \times 3 n_{\mathrm{seg}}$ matrix, $u_{\mathrm{TOT}}$ is a $3 n_{\mathrm{seg}} \times 1$ vector, and $\boldsymbol{\sigma}_{\mathrm{TOT}}$ is a $6 n_{\mathrm{SAF}} \times 1$ vector, where $n_{\mathrm{SAF}}$ is the number of SAF segments and $n_{\mathrm{seg}}$ is the number of segments in the entire SCFS. For the case of self stress rate, we calculate

$$
\boldsymbol{\sigma}_{\mathrm{SAF}}=\mathbf{H}_{\mathrm{SAF}} u_{\mathrm{SAF}}
$$

where $\mathbf{H}_{\mathrm{SAF}}$, a $6 n_{\mathrm{SAF}} \times 3 n_{\mathrm{SAF}}$ matrix, and $u_{\mathrm{SAF}}$, a $3 n_{\mathrm{SAF}} \times 1$ vector, are subsets of the full combined constitutive/partial derivative matrix and slip rate vector, respectively, with entries corresponding to SAF segments.

The results in Figs. 1 and 2 of the main text are expressed as shear stressing rates, $\tau$, derived by rotating the full stress rate tensor onto each SAF segment's geometry, using the tensor transfor- 
mation matrices

$$
\begin{aligned}
& \mathbf{A}=\left[\begin{array}{ccc}
\cos (\theta) & \sin (\theta) & 0 \\
-\sin (\theta) & \cos (\theta) & 0 \\
0 & 0 & 1
\end{array}\right], \\
& \mathbf{B}=\left[\begin{array}{ccc}
\cos (\delta) & 0 & \sin (\delta) \\
0 & 1 & 0 \\
-\sin (\delta) & 0 & \cos (\delta)
\end{array}\right], \\
& \mathbf{C}=\left[\begin{array}{ccc}
\cos (\beta) & -\sin (\beta) & 0 \\
\sin (\beta) & \cos (\beta) & 0 \\
0 & 0 & 1
\end{array}\right],
\end{aligned}
$$

where $\theta$ is the segment strike, $\delta$ is the dip, and $\beta$ is the rake of slip (equal to $180^{\circ}$ for pure rightlateral slip). We determine $\tau$ and the fault normal stress, $\sigma_{\mathrm{n}}$, by extracting the $(2,3)$ and $(3,3)$ components, respectively, of the transformed stress rate tensor, $\bar{\sigma}$, which is given by

$$
\bar{\sigma}=\mathbf{C}^{\top} \mathbf{B}^{\top} \mathbf{A}^{\top} \sigma \mathbf{A B C}
$$

We define the shear stress rate difference (Fig. 2c) as

$$
\Delta \tau^{\prime}=\frac{\tau_{\mathrm{TOT}}-\tau_{\mathrm{SAF}}}{\tau_{\mathrm{TOT}}} \times 100
$$

the numerator of which reflects the rate of shear stress induced on the SAF by all other segments.

\section{COULOMB FAILURE STRESS RATE}

In addition to the shear stress accumulation rates presented in Fig. 2, we calculate Coulomb failure stress (CFS) accumulation rate, which reflects the balance between shear stress rate, $\tau$, and normal stress rate, $\sigma_{\mathrm{n}}$, modulated by the effective coefficient of friction, $\mu$ (King et al., 1994):

$$
\mathrm{CFS}=\tau-\mu \sigma_{\mathrm{n}}
$$

Because we calculate $\tau$ and $\sigma_{\mathrm{n}}$ for each SAF segment, CFS reflects the likelihood of right-lateral failure on that segment. We calculate CFS using an effective friction coefficient of $\mu=0.4$ (King et al., 1994). The patterns of self, total, and differential CFS (Fig. DR1) generally follow those of shear stress rate (Fig. 2).

\section{CHANGES IN STRESS RATE WITH DEPTH}

In Fig. 2, we show stress rates resolved on the SAF at the segment centroids, the depths of which are equal to half the locking depth. We also resolved shear stress on SAF planes at 25\% and $75 \%$ of the locking depth and find broadly similar results (Figs. DR2, DR3). At 25\% locking depth, stress rate magnitudes (Fig. DR2) are generally 15-20\% lower than those of the 50\% locking depth case (Fig. 2), and $\sim 60-70 \%$ higher at 75\% locking depth (Fig. DR3). Stress rate differences are slightly larger for the $25 \%$ case (up to $\Delta \tau^{\prime}=47 \%$ along the San Bernardino section away from 
section junctions) (Fig. DR2c) and smaller for the 75\% case (Fig. DR3c). These relationships indicate that fault system interaction is more effective in modulating stress close to the surface than at depth.

\section{REFERENCES CITED}

Biasi, G., and R. Weldon (2009), San Andreas Fault Rupture Scenarios from Multiple Paleoseismic Records: Stringing Pearls, Bulletin of the Seismological Society of America, 99(2A), 471-498, doi: $10.1785 / 0120080287$.

Hammond, W. C., and W. Thatcher (2005), Northwest Basin and Range tectonic deformation observed with the Global Positioning System, 1999-2003, Journal of Geophysical Research, 110, B10405, doi:10.1029/2005JB003678.

King, G. C., R. S. Stein, and J. Lin (1994), Static stress changes and the triggering of earthquakes, Bulletin of the Seismological Society of America, 84(3), 935-953.

McCaffrey, R. (2005), Block kinematics of the Pacific-North America plate boundary in the southwestern United States from inversion of GPS, seismological, and geologic data, Journal of Geophysical Research, 110(B7), B07401, doi:10.1029/2004JB003307.

McClusky, S. C., S. C. Bjornstad, B. H. Hager, R. W. King, B. J. Meade, M. M. Miller, F. C. Monastero, and B. J. Souter (2001), Present day kinematics of the Eastern California Shear Zone from a geodetically constrained block model, Geophysical Research Letters, 28(17), 3369-3372.

Meade, B. J. (2007), Algorithms for the calculation of exact displacements, strains, and stresses for triangular dislocation elements in a uniform elastic half space, Computers and Geosciences, 33, 1064-1075, doi:10.1016/j.cageo.2006.12.003.

Meade, B. J., and J. P. Loveless (2009), Block modeling with connected fault network geometries and a linear elastic coupling estimator in spherical coordinates, Bulletin of the Seismological Society of America, 99(6), 3124-3139, doi:10.1785/0120090088.

Okada, Y. (1992), Internal deformation due to shear and tensile faults in a half-space, Bulletin of the Seismological Society of America, 82(2), 1018-1040.

Plate Boundary Observatory network velocity field (2008), http://pboweb.unavco.org/.

Plesch, A., J. Shaw, C. Benson, W. Bryant, S. Carena, M. Cooke, J. Dolan, G. Fuis, E. Gath, L. Grant, et al. (2007), Community Fault Model (CFM) for Southern California, Bulletin of the Seismological Society of America, 97(6), 1793-1802, doi:10.1785/0120050211.

Shen, Z., D. Agnew, R. King, D. Dong, T. Herring, M. Wang, H. Johnson, G. Anderson, R. Nikolaidis, M. van Domselaar, K. Hudnut, and D. Jackson (2003), SCEC Crustal Motion Map, Version 3.0, http://epicenter.usc.edu/cmm3/. 
Williams, T. B., H. M. Kelsey, and J. T. Freymueller (2006), GPS-derived strain in northwestern California: Termination of the San Andreas fault system and convergence of the Sierra NevadaGreat Valley block contribute to southern Cascadia forearc contraction, Tectonophysics, 413(34), 171-184, doi:10.1016/j.tecto.2005.10.047. 

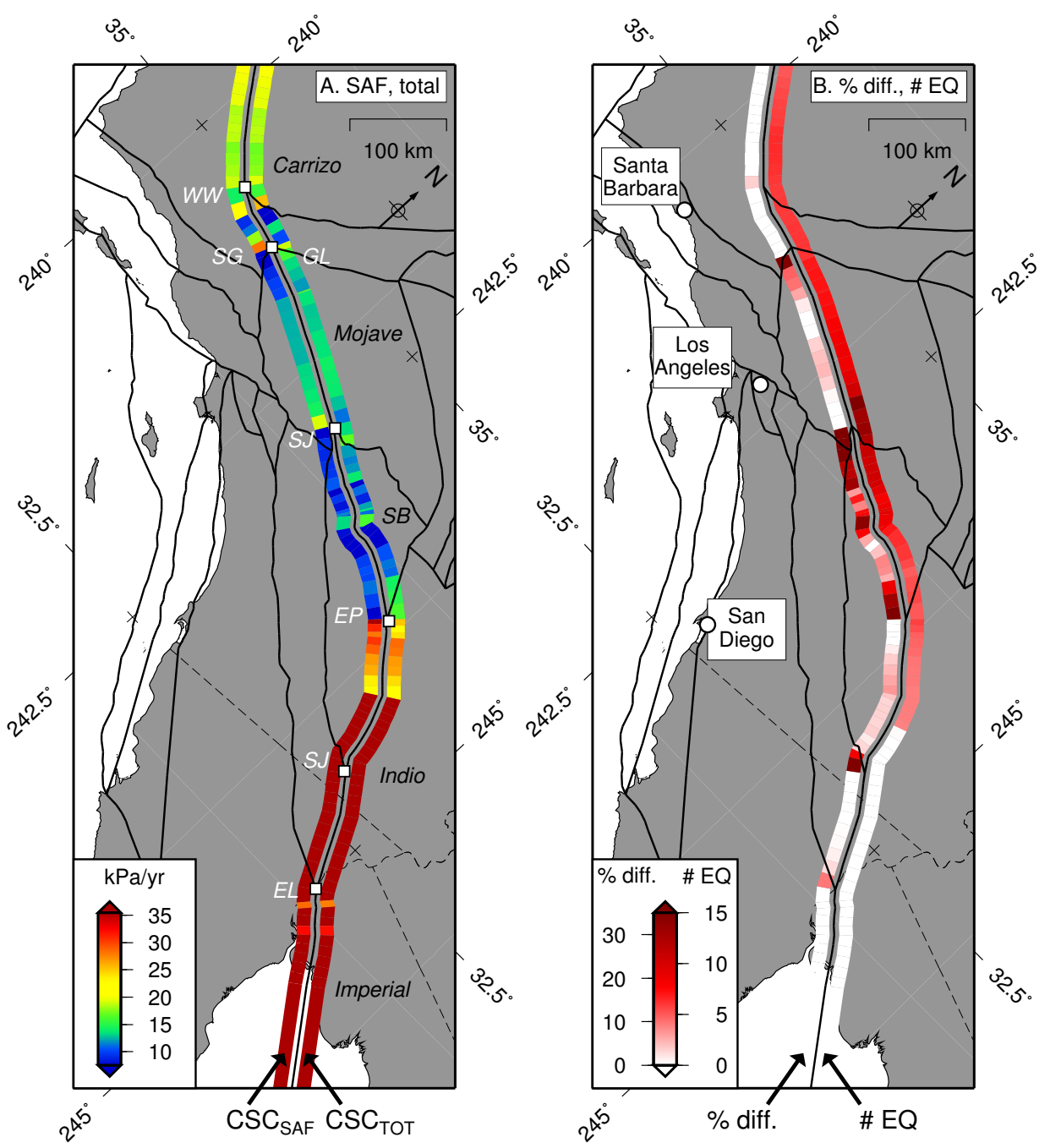

Figure DR1: Interseismic Coulomb failure stress (CFS) accumation rate resolved on the San Andreas fault (SAF). CFS rate calculated analytically (Okada, 1992) from the slip rates from our kinematically consistent block model, using an effective coefficient of friction of $\mu^{\prime}=0.4$. Two quantities are plotted in each panel, one to the left (southwest) and one to the right (northeast) of the SAF trace, as labeled at the bottom of the panel. A) Self CFS rate due to slip on SAF segments alone, $\mathrm{CSC}_{\mathrm{SAF}}$, and total $\mathrm{CFS}$ rate due to slip on all faults, $\mathrm{CSC}_{\mathrm{TOT}}$. Fault junctions are labeled in white; WW: White Wolf, GL: Garlock, SG: San Gabriel, SJ: San Jacinto, NF: North Frontal, EP: Eureka Peak, EL: Elsinore. Junctions bound labeled sections of the SAF (black italics); SB: San Bernardino. B) Difference between total and self-stress, normalized by self-stress $\left(\Delta \mathrm{CSC}^{\prime}=\left(\mathrm{CSC}_{\mathrm{TOT}}-\mathrm{CSC}_{\mathrm{SAF}}\right) / \mathrm{CSC}_{\mathrm{SAF}} \times 100\right.$, "\% diff." $)$, and mean number of paleoseismic earthquakes (“\# EQ") on each segment, from the rupture models of Biasi and Weldon (2009). 


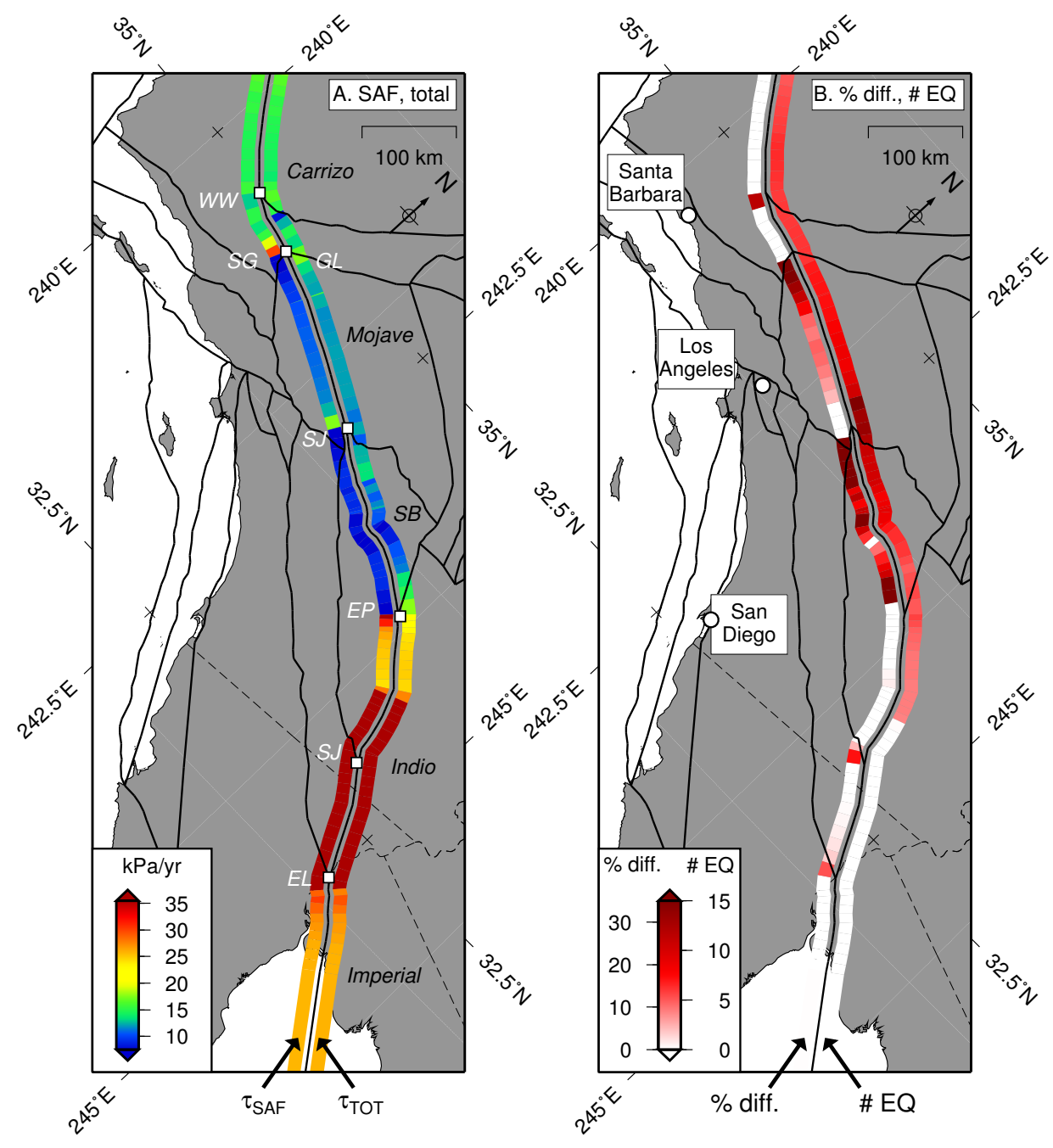

Figure DR2: Interseismic shear stressing rates calculated analytically (Okada, 1992) from the slip rates from our kinematically consistent block model. In contrast to Fig. 3 of the main text, in which shear stress was calculated at half the locking depth of each segment, values shown here reflect shear stress at $25 \%$ locking depth. A) Self shear stress rate due to slip on San Andreas fault (SAF) segments alone, $\tau_{\mathrm{SAF}}$, and total shear stress rate due to slip on all faults, $\tau_{\mathrm{TOT}}$. B) Difference between total and self-stress, normalized by self-stress $\left(\Delta \tau^{\prime}=\left(\tau_{\mathrm{TOT}}-\tau_{\mathrm{SAF}}\right) / \tau_{\mathrm{SAF}} \times 100\right)$, and mean number of paleoseismic earthquakes on each segment, from the rupture models of Biasi and Weldon (2009). Labels and abbreviations are defined in Fig. DR1 caption. 


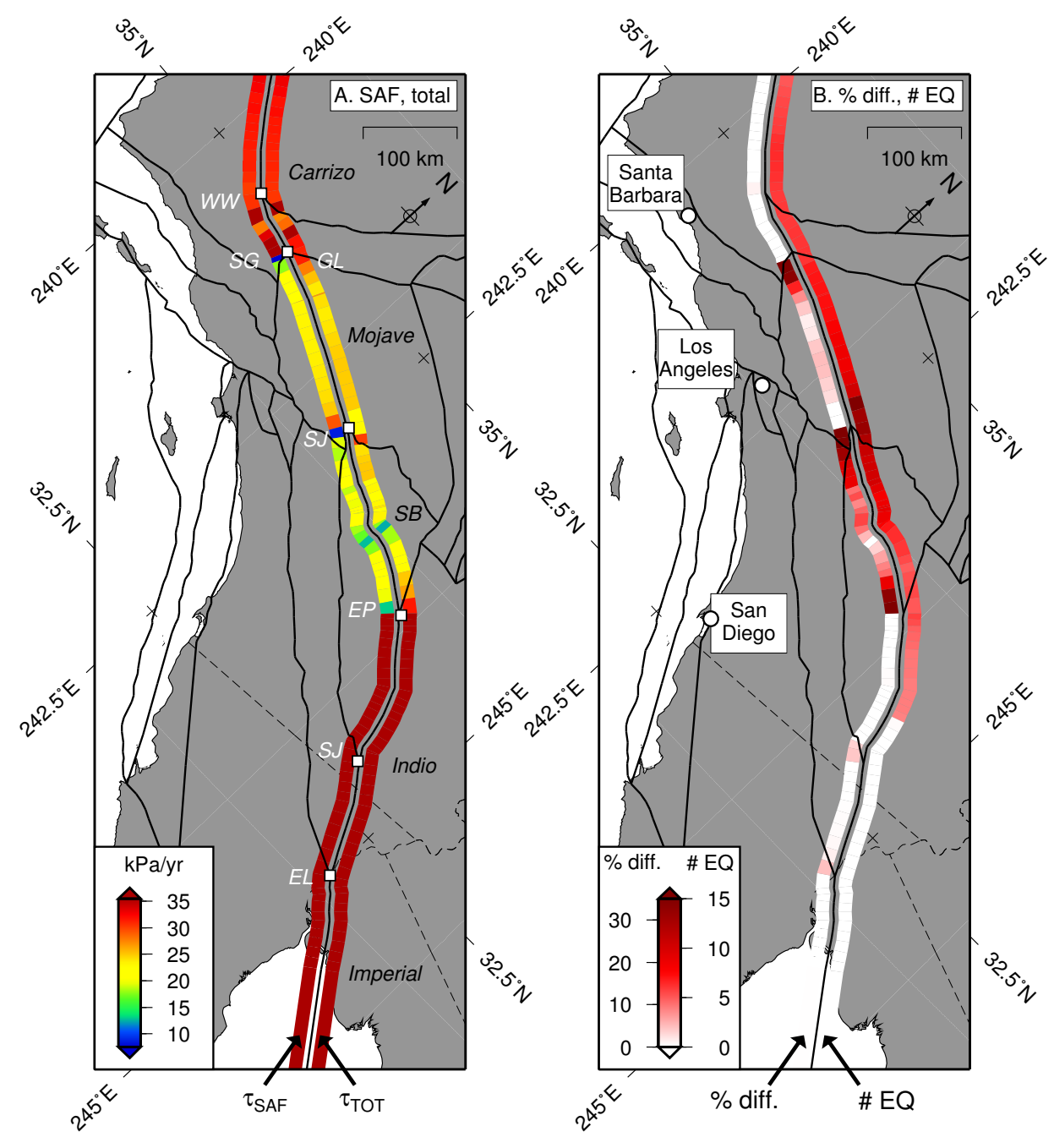

Figure DR3: Same as Fig. DR2, but shear stress rate is calculated at 75\% locking depth on each segment. 
Table DR1. Geometric parameters and estimated slip rates for southern California fault segments in our block model. Each named fault is divided into a number of segments (\#), with endpoints given as (Lon-1, Lat-1) and (Lon-2, Lat-2), length as L (km), locking depth as $Z$ $(\mathrm{km})$, strike, and dip (following the right-hand rule). We estimate strike slip, U_s (with uncertainty S_S), and dip slip, U_d (with uncertainty S_d), on dipping faults, and U_S and tensile slip, U_t (with uncertainty $S_{-} t$ ), on vertical faults. Segment names preceded by a letter label correspond to slip rates shown in Fig. $1 \mathrm{~b}$.

\# Lon-1 Lat-1 Lon-2 Lat-2 L(km) Z(km) strike dip U_s S_s U_d S_d U_t S_t

\section{a. SAF Carrizo}

$\begin{array}{llllllllllllllll}1 & 239.815 & 35.639 & 239.869 & 35.583 & 7.908 & 22.0 & 141.8 & 90 & 31.5 & 0.2 & 0.0 & 0.0 & -0.0 & 0.3\end{array}$

$\begin{array}{llllllllllllllll}2 & 239.869 & 35.583 & 239.923 & 35.528 & 7.824 & 22.0 & 141.2 & 90 & 31.5 & 0.2 & 0.0 & 0.0 & -0.1 & 0.3\end{array}$

$\begin{array}{lllllllllllllll}3 & 239.923 & 35.528 & 239.977 & 35.473 & 7.826 & 22.0 & 141.2 & 90 & 31.5 & 0.2 & 0.0 & 0.0 & 0.0 & 0.3\end{array}$

$\begin{array}{llllllllllllllll}4 & 239.977 & 35.473 & 240.031 & 35.418 & 7.828 & 22.0 & 141.2 & 90 & 31.5 & 0.2 & 0.0 & 0.0 & 0.2 & 0.3\end{array}$

$\begin{array}{lllllllllllllllll}5 & 240.031 & 35.418 & 240.085 & 35.362 & 7.917 & 22.0 & 141.7 & 90 & 31.4 & 0.2 & 0.0 & 0.0 & 0.7 & 0.3\end{array}$

$\begin{array}{lllllllllllllll}6 & 240.085 & 35.362 & 240.140 & 35.307 & 7.889 & 22.0 & 140.7 & 90 & 31.5 & 0.2 & 0.0 & 0.0 & 0.3 & 0.4\end{array}$

$\begin{array}{lllllllllllllll}7 & 240.140 & 35.307 & 240.188 & 35.264 & 6.467 & 22.0 & 137.5 & 90 & 31.4 & 0.2 & 0.0 & 0.0 & -1.2 & 0.4\end{array}$

$8 \begin{array}{lllllllllllllll}8 & 240.188 & 35.264 & 240.236 & 35.221 & 6.469 & 22.0 & 137.5 & 90 & 31.4 & 0.2 & 0.0 & 0.0 & -1.1 & 0.4\end{array}$

$\begin{array}{lllllllllllllllll}9 & 240.236 & 35.221 & 240.284 & 35.177 & 6.553 & 22.0 & 138.1 & 90 & 31.4 & 0.2 & 0.0 & 0.0 & -0.6 & 0.4\end{array}$

$\begin{array}{lllllllllllllllll}10 & 240.284 & 35.177 & 240.333 & 35.134 & 6.534 & 22.0 & 136.9 & 90 & 31.4 & 0.2 & 0.0 & 0.0 & -1.1 & 0.4\end{array}$

$\begin{array}{llllllllllllllll}11 & 240.333 & 35.134 & 240.399 & 35.085 & 8.109 & 22.0 & 132.1 & 90 & 31.2 & 0.2 & 0.0 & 0.0 & -3.5 & 0.4\end{array}$

$\begin{array}{lllllllllllllll}12 & 240.399 & 35.085 & 240.464 & 35.037 & 7.970 & 22.0 & 131.9 & 90 & 31.2 & 0.2 & 0.0 & 0.0 & -3.4 & 0.4\end{array}$

$\begin{array}{lllllllllllllll}13 & 240.464 & 35.037 & 240.529 & 34.988 & 8.047 & 22.0 & 132.5 & 90 & 31.2 & 0.2 & 0.0 & 0.0 & -2.9 & 0.4\end{array}$

$\begin{array}{lllllllllllllll}14 & 240.529 & 34.988 & 240.594 & 34.942 & 7.828 & 22.0 & 130.7 & 90 & 31.1 & 0.2 & 0.0 & 0.0 & -3.7 & 0.5\end{array}$

b. SAF White Wolf-Garlock

$\begin{array}{lllllllllllllll}1 & 240.594 & 34.942 & 240.692 & 34.903 & 9.945 & 25.0 & 115.8 & 90 & 28.5 & 0.4 & 0.0 & 0.0 & -8.4 & 0.7\end{array}$

$\begin{array}{llllllllllllllll}2 & 240.692 & 34.903 & 240.790 & 34.864 & 9.949 & 25.0 & 115.7 & 90 & 28.5 & 0.4 & 0.0 & 0.0 & -8.3 & 0.7\end{array}$

$\begin{array}{llllllllllllllll}3 & 240.790 & 34.864 & 240.863 & 34.855 & 6.750 & 15.0 & 98.5 & 90 & 24.8 & 0.4 & 0.0 & 0.0 & -16.2 & 0.7\end{array}$

$\begin{array}{llllllllllllllll}4 & 240.863 & 34.855 & 240.936 & 34.846 & 6.750 & 15.0 & 98.5 & 90 & 24.8 & 0.4 & 0.0 & 0.0 & -16.1 & 0.7\end{array}$

$\begin{array}{llllllllllllllll}5 & 240.936 & 34.846 & 241.019 & 34.828 & 7.850 & 15.0 & 104.7 & 90 & 26.4 & 0.4 & 0.0 & 0.0 & -13.2 & 0.7\end{array}$

$\begin{array}{llllllllllllllll}6 & 241.019 & 34.828 & 241.103 & 34.811 & 7.913 & 15.0 & 103.8 & 90 & 26.1 & 0.4 & 0.0 & 0.0 & -13.5 & 0.7\end{array}$

\section{SAF Mojave}

$\begin{array}{lllllllllllllllll}1 & 241.103 & 34.811 & 241.144 & 34.792 & 4.303 & 15.0 & 119.3 & 90 & 16.4 & 0.8 & 0.0 & 0.0 & -1.7 & 1.1\end{array}$

$\begin{array}{lllllllllllllll}2 & 241.144 & 34.792 & 241.231 & 34.769 & 8.362 & 15.0 & 107.7 & 90 & 15.7 & 0.7 & 0.0 & 0.0 & -4.9 & 1.1\end{array}$

$\begin{array}{lllllllllllllll}3 & 241.231 & 34.769 & 241.318 & 34.746 & 8.364 & 15.0 & 107.7 & 90 & 15.7 & 0.7 & 0.0 & 0.0 & -4.9 & 1.0\end{array}$

$\begin{array}{llllllllllllllll}4 & 241.318 & 34.746 & 241.406 & 34.724 & 8.421 & 15.0 & 106.8 & 90 & 15.7 & 0.7 & 0.0 & 0.0 & -5.1 & 1.0\end{array}$

$\begin{array}{lllllllllllllll}5 & 241.406 & 34.724 & 241.493 & 34.701 & 8.368 & 15.0 & 107.7 & 90 & 15.7 & 0.7 & 0.0 & 0.0 & -4.8 & 0.9\end{array}$

$\begin{array}{lllllllllllllll}6 & 241.493 & 34.701 & 241.498 & 34.699 & 0.509 & 15.0 & 115.8 & 90 & 16.3 & 0.8 & 0.0 & 0.0 & -2.5 & 0.8\end{array}$

$\begin{array}{llllllllllllllll}7 & 241.498 & 34.699 & 241.597 & 34.662 & 9.958 & 15.0 & 114.3 & 90 & 16.2 & 0.8 & 0.0 & 0.0 & -3.0 & 0.8\end{array}$

$\begin{array}{llllllllllllllll}8 & 241.597 & 34.662 & 241.696 & 34.624 & 10.008 & 15.0 & 114.9 & 90 & 16.2 & 0.8 & 0.0 & 0.0 & -2.8 & 0.7\end{array}$

$\begin{array}{llllllllllllllll}9 & 241.696 & 34.624 & 241.795 & 34.587 & 9.965 & 15.0 & 114.3 & 90 & 16.2 & 0.8 & 0.0 & 0.0 & -2.9 & 0.6\end{array}$

$\begin{array}{lllllllllllllll}10 & 241.795 & 34.587 & 241.894 & 34.549 & 10.015 & 15.0 & 114.9 & 90 & 16.2 & 0.8 & 0.0 & 0.0 & -2.7 & 0.6\end{array}$

$\begin{array}{lllllllllllllll}11 & 241.894 & 34.549 & 241.993 & 34.512 & 9.973 & 15.0 & 114.3 & 90 & 16.2 & 0.8 & 0.0 & 0.0 & -2.9 & 0.6\end{array}$

$\begin{array}{lllllllllllllll}12 & 241.993 & 34.512 & 242.088 & 34.472 & 9.789 & 15.0 & 116.9 & 90 & 16.3 & 0.8 & 0.0 & 0.0 & -2.1 & 0.6\end{array}$

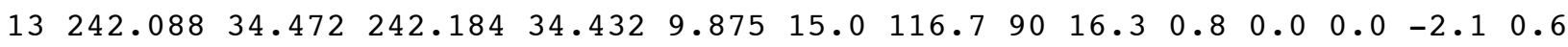

$\begin{array}{llllllllllllllll}14 & 242.184 & 34.432 & 242.279 & 34.391 & 9.847 & 15.0 & 117.5 & 90 & 16.3 & 0.8 & 0.0 & 0.0 & -1.8 & 0.7\end{array}$

$\begin{array}{llllllllllllllll}15 & 242.279 & 34.391 & 242.375 & 34.351 & 9.882 & 15.0 & 116.7 & 90 & 16.3 & 0.8 & 0.0 & 0.0 & -2.1 & 0.8\end{array}$

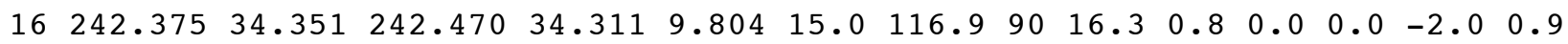

\section{d. SAF San Bernadino}

$\begin{array}{llllllllllllllllll}1 & 242.470 & 34.311 & 242.549 & 34.273 & 8.407 & 15.0 & 120.1 & 90 & 9.9 & 0.3 & 0.0 & 0.0 & -3.3 & 0.6\end{array}$

$\begin{array}{lllllllllllllllll}2 & 242.549 & 34.273 & 242.613 & 34.232 & 7.446 & 15.0 & 127.6 & 90 & 10.2 & 0.3 & 0.0 & 0.0 & -1.9 & 0.5\end{array}$

$\begin{array}{llllllllllllllll}3 & 242.613 & 34.232 & 242.695 & 34.188 & 8.996 & 10.0 & 122.8 & 90 & 10.0 & 0.3 & 0.0 & 0.0 & -2.6 & 0.5\end{array}$

$\begin{array}{llllllllllllllll}4 & 242.695 & 34.188 & 242.754 & 34.161 & 6.210 & 10.0 & 118.8 & 90 & 9.8 & 0.3 & 0.0 & 0.0 & -3.3 & 0.5\end{array}$

$\begin{array}{llllllllllllllllll}5 & 242.754 & 34.161 & 242.813 & 34.137 & 6.058 & 10.0 & 116.1 & 90 & 9.7 & 0.3 & 0.0 & 0.0 & -3.6 & 0.4\end{array}$

$\begin{array}{llllllllllllllll}6 & 242.813 & 34.137 & 242.883 & 34.102 & 7.535 & 10.0 & 121.0 & 90 & 9.9 & 0.3 & 0.0 & 0.0 & -2.7 & 0.4\end{array}$

$\begin{array}{lllllllllllllll}7 & 242.883 & 34.102 & 242.930 & 34.091 & 4.506 & 10.0 & 105.7 & 90 & 8.9 & 0.3 & 0.0 & 0.0 & -5.2 & 0.4\end{array}$ 
\# Lon-1 Lat-1 Lon-2 Lat-2 L(km) $\mathrm{z}(\mathrm{km})$ strike dip U_S S_S U_d S_d U_t S_t

$\begin{array}{llllllllllllllll}8 & 242.930 & 34.091 & 242.985 & 34.070 & 5.585 & 10.0 & 114.6 & 90 & 9.6 & 0.3 & 0.0 & 0.0 & -3.7 & 0.4\end{array}$ $\begin{array}{llllllllllllllll}9 & 242.985 & 34.070 & 243.053 & 34.049 & 6.696 & 10.0 & 110.3 & 90 & 9.3 & 0.3 & 0.0 & 0.0 & -4.3 & 0.4\end{array}$ $\begin{array}{llllllllllllllll}10 & 243.053 & 34.049 & 243.083 & 34.034 & 3.231 & 10.0 & 121.0 & 90 & 9.9 & 0.3 & 0.0 & 0.0 & -2.4 & 0.3\end{array}$ $\begin{array}{lllllllllllllllll}11 & 243.083 & 34.034 & 243.098 & 34.026 & 1.645 & 10.0 & 122.6 & 90 & 10.0 & 0.3 & 0.0 & 0.0 & -2.1 & 0.3\end{array}$ $\begin{array}{llllllllllllllll}12 & 243.098 & 34.026 & 243.113 & 34.019 & 1.588 & 10.0 & 119.3 & 90 & 9.8 & 0.3 & 0.0 & 0.0 & -2.7 & 0.3\end{array}$ $\begin{array}{llllllllllllllll}13 & 243.113 & 34.019 & 243.177 & 33.959 & 8.903 & 10.0 & 138.4 & 90 & 10.2 & 0.3 & 0.0 & 0.0 & 0.7 & 0.2\end{array}$ $\begin{array}{llllllllllllllll}14 & 243.177 & 33.959 & 243.202 & 33.951 & 2.475 & 10.0 & 111.0 & 90 & 9.4 & 0.3 & 0.0 & 0.0 & -4.0 & 0.3\end{array}$ $\begin{array}{lllllllllllllll}15 & 243.202 & 33.951 & 243.246 & 33.945 & 4.121 & 10.0 & 99.3 & 90 & 8.4 & 0.2 & 0.0 & 0.0 & -5.7 & 0.3\end{array}$ $\begin{array}{llllllllllllllll}16 & 243.246 & 33.945 & 243.307 & 33.950 & 5.666 & 10.0 & 84.4 & 90 & 6.6 & 0.2 & 0.0 & 0.0 & -7.7 & 0.3\end{array}$ $\begin{array}{lllllllllllllllll}17 & 243.307 & 33.950 & 243.406 & 33.935 & 9.302 & 10.0 & 100.3 & 90 & 8.4 & 0.2 & 0.0 & 0.0 & -5.4 & 0.3\end{array}$ $\begin{array}{lllllllllllllll}18 & 243.406 & 33.935 & 243.502 & 33.900 & 9.689 & 10.0 & 113.6 & 90 & 9.5 & 0.3 & 0.0 & 0.0 & -3.2 & 0.2\end{array}$ $\begin{array}{llllllllllllllll}19 & 243.502 & 33.900 & 243.556 & 33.876 & 5.660 & 10.0 & 118.0 & 90 & 9.7 & 0.3 & 0.0 & 0.0 & -2.4 & 0.2\end{array}$

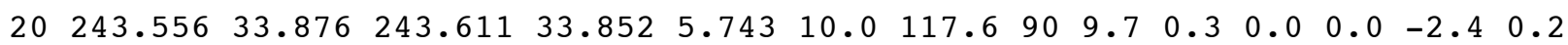
$\begin{array}{lllllllllllllll}21 & 243.611 & 33.852 & 243.699 & 33.801 & 9.918 & 10.0 & 124.8 & 90 & 9.9 & 0.3 & 0.0 & 0.0 & -1.1 & 0.2\end{array}$ $\begin{array}{lllllllllllllll}22 & 243.699 & 33.801 & 243.788 & 33.750 & 9.998 & 10.0 & 124.4 & 90 & 9.9 & 0.3 & 0.0 & 0.0 & -1.0 & 0.2\end{array}$ $\begin{array}{lllllllllllllll}23 & 243.788 & 33.750 & 243.876 & 33.699 & 9.926 & 10.0 & 124.7 & 90 & 9.9 & 0.3 & 0.0 & 0.0 & -0.8 & 0.2\end{array}$

\section{e. SAF Indio}

$\begin{array}{lllllllllllllll}1 & 243.876 & 33.699 & 243.903 & 33.671 & 3.989 & 10.0 & 141.1 & 90 & 24.1 & 0.2 & 0.0 & 0.0 & -4.4 & 0.2\end{array}$

$\begin{array}{llllllllllllllll}2 & 243.903 & 33.671 & 243.945 & 33.638 & 5.346 & 10.0 & 133.2 & 90 & 23.3 & 0.2 & 0.0 & 0.0 & -7.9 & 0.2\end{array}$

$\begin{array}{llllllllllllllll}3 & 243.945 & 33.638 & 243.976 & 33.612 & 4.073 & 10.0 & 135.1 & 90 & 23.5 & 0.2 & 0.0 & 0.0 & -7.4 & 0.2\end{array}$

$\begin{array}{llllllllllllllll}4 & 243.976 & 33.612 & 244.019 & 33.567 & 6.391 & 10.0 & 141.3 & 90 & 24.2 & 0.2 & 0.0 & 0.0 & -5.1 & 0.2\end{array}$

$\begin{array}{lllllllllllllllll}5 & 244.019 & 33.567 & 244.063 & 33.522 & 6.451 & 10.0 & 140.7 & 90 & 24.1 & 0.2 & 0.0 & 0.0 & -5.7 & 0.2\end{array}$

$\begin{array}{lllllllllllllll}6 & 244.063 & 33.522 & 244.117 & 33.472 & 7.479 & 10.0 & 137.8 & 90 & 23.8 & 0.2 & 0.0 & 0.0 & -7.2 & 0.2\end{array}$

$\begin{array}{llllllllllllllll}7 & 244.117 & 33.472 & 244.171 & 33.423 & 7.399 & 10.0 & 137.3 & 90 & 23.7 & 0.2 & 0.0 & 0.0 & -7.9 & 0.3\end{array}$

$\begin{array}{lllllllllllllll}8 & 244.171 & 33.423 & 244.228 & 33.376 & 7.436 & 10.0 & 134.5 & 90 & 23.3 & 0.2 & 0.0 & 0.0 & -9.4 & 0.3\end{array}$

$\begin{array}{lllllllllllllllll}9 & 244.228 & 33.376 & 244.286 & 33.329 & 7.505 & 10.0 & 134.0 & 90 & 23.2 & 0.2 & 0.0 & 0.0 & -10.0 & 0.3\end{array}$

$\begin{array}{llllllllllllllll}10 & 244.286 & 33.329 & 244.308 & 33.286 & 5.191 & 10.0 & 156.7 & 90 & 25.3 & 0.2 & 0.0 & 0.0 & -0.6 & 0.3\end{array}$

$\begin{array}{lllllllllllllllll}11 & 244.308 & 33.286 & 244.330 & 33.243 & 5.191 & 10.0 & 156.7 & 90 & 25.3 & 0.2 & 0.0 & 0.0 & -0.9 & 0.3\end{array}$

$\begin{array}{lllllllllllllll}12 & 244.330 & 33.243 & 244.352 & 33.197 & 5.499 & 5.0 & 158.1 & 90 & 25.4 & 0.2 & 0.0 & 0.0 & -0.5 & 0.4\end{array}$

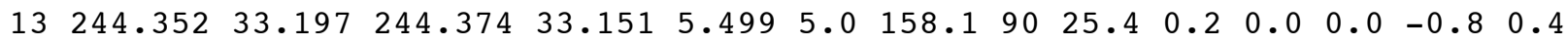

$\begin{array}{llllllllllllllll}14 & 244.374 & 33.151 & 244.399 & 33.084 & 7.788 & 5.0 & 162.6 & 90 & 25.4 & 0.2 & 0.0 & 0.0 & 0.8 & 0.4\end{array}$

$\begin{array}{lllllllllllllll}15 & 244.399 & 33.084 & 244.423 & 33.017 & 7.761 & 5.0 & 163.2 & 90 & 25.4 & 0.2 & 0.0 & 0.0 & 0.7 & 0.4\end{array}$

$\begin{array}{lllllllllllllll}16 & 244.423 & 33.017 & 244.435 & 32.958 & 6.639 & 2.5 & 170.3 & 90 & 25.1 & 0.2 & 0.0 & 0.0 & 3.4 & 0.4\end{array}$

$\begin{array}{llllllllllllllll}17 & 244.435 & 32.958 & 244.446 & 32.899 & 6.624 & 2.5 & 171.1 & 90 & 25.1 & 0.2 & 0.0 & 0.0 & 3.4 & 0.4\end{array}$

$\begin{array}{llllllllllllllll}18 & 244.446 & 32.899 & 244.465 & 32.872 & 3.482 & 2.5 & 149.3 & 90 & 24.5 & 0.2 & 0.0 & 0.0 & -6.3 & 0.5\end{array}$

$\begin{array}{llllllllllllllll}19 & 244.465 & 32.872 & 244.522 & 32.807 & 8.969 & 2.5 & 143.5 & 90 & 23.7 & 0.2 & 0.0 & 0.0 & -9.1 & 0.5\end{array}$

f. SAF S. Indio

$\begin{array}{llllllllllllllll}1 & 244.522 & 32.807 & 244.571 & 32.753 & 7.546 & 5.0 & 142.5 & 90 & 33.7 & 0.2 & 0.0 & 0.0 & 6.9 & 0.3\end{array}$

$\begin{array}{llllllllllllllll}2 & 244.571 & 32.753 & 244.621 & 32.700 & 7.518 & 5.0 & 141.4 & 90 & 33.8 & 0.2 & 0.0 & 0.0 & 6.4 & 0.3\end{array}$

$\begin{array}{llllllllllllllll}3 & 244.621 & 32.700 & 244.670 & 32.646 & 7.549 & 5.0 & 142.5 & 90 & 33.7 & 0.2 & 0.0 & 0.0 & 7.2 & 0.3\end{array}$

$\begin{array}{llllllllllllllll}4 & 244.670 & 32.646 & 244.720 & 32.592 & 7.608 & 5.0 & 141.9 & 90 & 33.8 & 0.2 & 0.0 & 0.0 & 7.0 & 0.3\end{array}$

$\begin{array}{llllllllllllllll}5 & 244.720 & 32.592 & 244.768 & 32.516 & 9.558 & 5.0 & 151.8 & 90 & 32.1 & 0.2 & 0.0 & 0.0 & 12.9 & 0.3\end{array}$

$\begin{array}{llllllllllllllll}6 & 244.768 & 32.516 & 244.816 & 32.440 & 9.560 & 5.0 & 151.8 & 90 & 32.1 & 0.2 & 0.0 & 0.0 & 13.1 & 0.4\end{array}$

$\begin{array}{lllllllllllllll}7 & 244.816 & 32.440 & 244.863 & 32.364 & 9.517 & 5.0 & 152.3 & 90 & 32.0 & 0.2 & 0.0 & 0.0 & 13.5 & 0.4\end{array}$

$\begin{array}{llllllllllllllll}8 & 244.863 & 32.364 & 244.911 & 32.288 & 9.563 & 5.0 & 151.8 & 90 & 32.1 & 0.2 & 0.0 & 0.0 & 13.4 & 0.4\end{array}$

$\begin{array}{llllllllllllllll}9 & 244.911 & 32.288 & 244.959 & 32.212 & 9.565 & 5.0 & 151.8 & 90 & 32.1 & 0.2 & 0.0 & 0.0 & 13.6 & 0.4\end{array}$

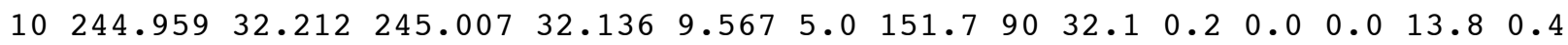

g. SAF Imperial

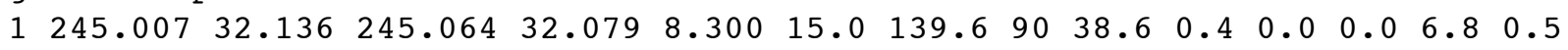

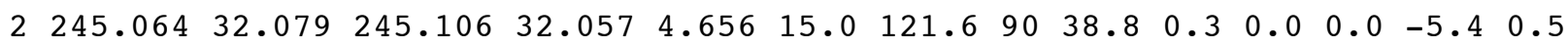

$\begin{array}{llllllllllllllllll}3 & 245.106 & 32.057 & 245.147 & 32.013 & 6.229 & 15.0 & 141.5 & 90 & 38.3 & 0.4 & 0.0 & 0.0 & 8.3 & 0.5\end{array}$

$\begin{array}{llllllllllllllllll}4 & 245.147 & 32.013 & 245.199 & 31.965 & 7.244 & 15.0 & 137.3 & 90 & 38.8 & 0.4 & 0.0 & 0.0 & 5.5 & 0.5\end{array}$

$\begin{array}{llllllllllllllllll}5 & 245.199 & 31.965 & 245.251 & 31.926 & 6.548 & 15.0 & 131.3 & 90 & 39.2 & 0.4 & 0.0 & 0.0 & 1.6 & 0.6\end{array}$

$\begin{array}{lllllllllllllllll}6 & 245.251 & 31.926 & 245.298 & 31.876 & 7.107 & 15.0 & 141.3 & 90 & 38.3 & 0.4 & 0.0 & 0.0 & 8.5 & 0.5\end{array}$

$\begin{array}{lllllllllllllllll}7 & 245.298 & 31.876 & 245.344 & 31.826 & 7.049 & 15.0 & 141.8 & 90 & 38.2 & 0.4 & 0.0 & 0.0 & 9.0 & 0.6\end{array}$ 
\# Lon-1 Lat-1 Lon-2 Lat-2 L(km) $\mathrm{z}(\mathrm{km})$ strike dip U_S S_S U_d S_d U_t S_t

$\begin{array}{lllllllllllllllll}8 & 245.344 & 31.826 & 245.389 & 31.769 & 7.623 & 15.0 & 146.0 & 90 & 37.5 & 0.4 & 0.0 & 0.0 & 11.8 & 0.6\end{array}$ $\begin{array}{llllllllllllllll}9 & 245.389 & 31.769 & 245.434 & 31.712 & 7.624 & 15.0 & 146.0 & 90 & 37.5 & 0.4 & 0.0 & 0.0 & 12.0 & 0.6\end{array}$ $\begin{array}{lllllllllllllll}10 & 245.434 & 31.712 & 245.479 & 31.655 & 7.626 & 15.0 & 146.0 & 90 & 37.5 & 0.4 & 0.0 & 0.0 & 12.1 & 0.6\end{array}$

h. San Jacinto

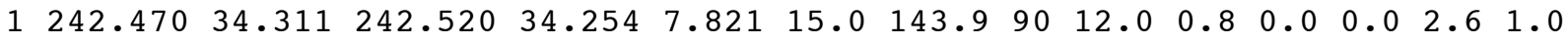

$2 \begin{array}{lllllllllllllll}2 & 242.520 & 34.254 & 242.566 & 34.232 & 4.890 & 15.0 & 119.9 & 90 & 12.1 & 0.7 & 0.0 & 0.0 & -2.1 & 1.1\end{array}$

$\begin{array}{llllllllllllllll}3 & 242.566 & 34.232 & 242.614 & 34.195 & 6.034 & 15.0 & 132.8 & 90 & 12.3 & 0.8 & 0.0 & 0.0 & 1.0 & 1.1\end{array}$

$\begin{array}{llllllllllllllll}4 & 242.614 & 34.195 & 242.636 & 34.161 & 4.282 & 15.0 & 151.7 & 90 & 13.3 & 0.4 & 0.0 & 0.0 & 2.2 & 0.1\end{array}$

$\begin{array}{lllllllllllllll}5 & 242.636 & 34.161 & 242.656 & 34.120 & 4.908 & 15.0 & 157.9 & 90 & 13.0 & 0.4 & 0.0 & 0.0 & 3.9 & 0.1\end{array}$

$\begin{array}{llllllllllllllll}6 & 242.656 & 34.120 & 242.778 & 34.008 & 16.769 & 15.0 & 137.8 & 90 & 13.6 & 0.4 & 0.0 & 0.0 & 0.0 & 0.0\end{array}$

$\begin{array}{lllllllllllllllll}7 & 242.778 & 34.008 & 242.928 & 33.894 & 18.766 & 15.0 & 132.3 & 90 & 13.6 & 0.4 & 0.0 & 0.0 & -0.0 & 0.0\end{array}$

$\begin{array}{lllllllllllllll}8 & 242.928 & 33.894 & 242.988 & 33.847 & 7.616 & 15.0 & 133.2 & 90 & 13.6 & 0.4 & 0.0 & 0.0 & 1.2 & 0.0\end{array}$

$\begin{array}{lllllllllllllllll}9 & 242.988 & 33.847 & 243.073 & 33.791 & 10.025 & 15.0 & 128.3 & 90 & 13.7 & 0.4 & 0.0 & 0.0 & 0.6 & 0.0\end{array}$

$\begin{array}{lllllllllllllll}10 & 243.073 & 33.791 & 243.104 & 33.743 & 6.049 & 15.0 & 151.6 & 90 & 12.1 & 0.3 & 0.0 & 0.0 & 6.5 & 0.2\end{array}$

$\begin{array}{lllllllllllllllll}11 & 243.104 & 33.743 & 243.159 & 33.702 & 6.831 & 15.0 & 131.7 & 90 & 13.7 & 0.4 & 0.0 & 0.0 & 2.5 & 0.1\end{array}$

$\begin{array}{llllllllllllllll}12 & 243.159 & 33.702 & 243.451 & 33.513 & 34.261 & 15.0 & 127.6 & 90 & 13.9 & 0.4 & 0.0 & 0.0 & 3.0 & 0.1\end{array}$

$\begin{array}{llllllllllllllll}13 & 243.451 & 33.513 & 243.457 & 33.471 & 4.692 & 15.0 & 173.2 & 90 & 6.8 & 0.2 & 0.0 & 0.0 & 13.0 & 0.4\end{array}$

$\begin{array}{lllllllllllllllll}14 & 243.457 & 33.471 & 243.530 & 33.422 & 8.695 & 15.0 & 128.7 & 90 & 14.1 & 0.4 & 0.0 & 0.0 & 5.0 & 0.1\end{array}$

$\begin{array}{lllllllllllllllll}15 & 243.530 & 33.422 & 243.717 & 33.265 & 24.622 & 15.0 & 135.0 & 90 & 13.4 & 0.4 & 0.0 & 0.0 & 7.7 & 0.2\end{array}$

$\begin{array}{llllllllllllllll}16 & 243.717 & 33.265 & 243.795 & 33.208 & 9.634 & 15.0 & 131.0 & 90 & 14.0 & 0.4 & 0.0 & 0.0 & 8.0 & 0.2\end{array}$

$\begin{array}{lllllllllllllllll}17 & 243.795 & 33.208 & 243.836 & 33.184 & 4.658 & 15.0 & 124.8 & 90 & 14.8 & 0.4 & 0.0 & 0.0 & 7.0 & 0.2\end{array}$

$\begin{array}{llllllllllllllll}18 & 243.836 & 33.184 & 243.871 & 33.145 & 5.419 & 15.0 & 142.9 & 90 & 11.8 & 0.3 & 0.0 & 0.0 & 11.6 & 0.3\end{array}$

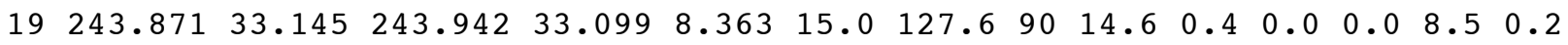

$\begin{array}{lllllllllllllll}20 & 243.942 & 33.099 & 243.989 & 33.031 & 8.726 & 15.0 & 149.8 & 90 & 10.1 & 0.3 & 0.0 & 0.0 & 14.0 & 0.4\end{array}$

$\begin{array}{llllllllllllllll}21 & 243.989 & 33.031 & 244.057 & 33.003 & 7.072 & 15.0 & 116.0 & 90 & 16.4 & 0.5 & 0.0 & 0.0 & 6.5 & 0.2\end{array}$

$\begin{array}{lllllllllllllll}22 & 244.057 & 33.003 & 244.191 & 32.928 & 15.037 & 15.0 & 123.5 & 90 & 15.4 & 0.4 & 0.0 & 0.0 & 9.4 & 0.3\end{array}$

$\begin{array}{lllllllllllllll}23 & 244.191 & 32.928 & 244.302 & 32.886 & 11.381 & 15.0 & 114.1 & 90 & 16.8 & 0.5 & 0.0 & 0.0 & 7.7 & 0.2\end{array}$

$\begin{array}{llllllllllllllll}24 & 244.302 & 32.886 & 244.336 & 32.899 & 3.493 & 15.0 & 65.6 & 90 & 17.2 & 0.5 & 0.0 & 0.0 & -7.2 & 0.2\end{array}$

$\begin{array}{llllllllllllllll}25 & 244.336 & 32.899 & 244.423 & 32.851 & 9.728 & 10.0 & 123.2 & 90 & 15.1 & 0.4 & 0.0 & 0.0 & 11.1 & 0.3\end{array}$

$\begin{array}{llllllllllllllll}26 & 244.423 & 32.851 & 244.522 & 32.807 & 10.476 & 2.5 & 117.7 & 90 & 16.2 & 0.5 & 0.0 & 0.0 & 10.4 & 0.3\end{array}$

i. Elsinore

$\begin{array}{llllllllllllllll}1 & 242.191 & 34.131 & 242.223 & 34.082 & 6.185 & 15.0 & 151.5 & 90 & 1.0 & 1.7 & 0.0 & 0.0 & 4.1 & 0.9\end{array}$

$\begin{array}{lllllllllllllllll}2 & 242.223 & 34.082 & 242.255 & 34.033 & 6.186 & 15.0 & 151.5 & 90 & 1.0 & 1.7 & 0.0 & 0.0 & 3.0 & 0.8\end{array}$

$\begin{array}{llllllllllllllll}3 & 242.255 & 34.033 & 242.274 & 34.000 & 4.059 & 15.0 & 154.4 & 67 & 0.8 & 1.7 & -5.6 & 2.0 & 0.0 & 0.0\end{array}$

$\begin{array}{llllllllllllllll}4 & 242.274 & 34.000 & 242.292 & 33.967 & 4.021 & 15.0 & 155.6 & 67 & 0.8 & 1.7 & -3.8 & 2.2 & 0.0 & 0.0\end{array}$

$\begin{array}{lllllllllllllllll}5 & 242.292 & 33.967 & 242.352 & 33.909 & 8.495 & 15.0 & 139.2 & 61 & 1.1 & 1.7 & -0.2 & 2.3 & 0.0 & 0.0\end{array}$

$\begin{array}{lllllllllllllll}6 & 242.352 & 33.909 & 242.403 & 33.854 & 7.712 & 15.0 & 142.3 & 53 & 1.1 & 1.7 & 2.1 & 2.3 & 0.0 & 0.0\end{array}$

$\begin{array}{llllllllllllllll}7 & 242.403 & 33.854 & 242.452 & 33.815 & 6.268 & 15.0 & 133.6 & 90 & 0.8 & 1.6 & 0.0 & 0.0 & -2.6 & 1.8\end{array}$

$\begin{array}{lllllllllllllllll}8 & 242.452 & 33.815 & 242.499 & 33.782 & 5.687 & 15.0 & 130.1 & 90 & 4.1 & 0.5 & 0.0 & 0.0 & 0.0 & 0.0\end{array}$

$\begin{array}{lllllllllllllllll}9 & 242.499 & 33.782 & 242.561 & 33.734 & 7.832 & 15.0 & 132.8 & 90 & 4.1 & 0.5 & 0.0 & 0.0 & 0.2 & 0.0\end{array}$

$\begin{array}{llllllllllllllll}10 & 242.561 & 33.734 & 242.611 & 33.669 & 8.571 & 15.0 & 147.2 & 90 & 4.0 & 0.5 & 0.0 & 0.0 & 1.2 & 0.1\end{array}$

$\begin{array}{lllllllllllllllll}11 & 242.611 & 33.669 & 242.646 & 33.646 & 4.129 & 15.0 & 128.2 & 90 & 4.1 & 0.5 & 0.0 & 0.0 & -0.2 & 0.0\end{array}$

$\begin{array}{lllllllllllllllll}12 & 242.646 & 33.646 & 242.762 & 33.571 & 13.605 & 15.0 & 127.7 & 90 & 4.1 & 0.5 & 0.0 & 0.0 & -0.3 & 0.0\end{array}$

$\begin{array}{llllllllllllllll}13 & 242.762 & 33.571 & 242.804 & 33.519 & 6.963 & 15.0 & 145.9 & 90 & 4.0 & 0.5 & 0.0 & 0.0 & 1.0 & 0.1\end{array}$

$\begin{array}{lllllllllllllllll}14 & 242.804 & 33.519 & 242.844 & 33.487 & 5.139 & 15.0 & 133.7 & 90 & 4.1 & 0.5 & 0.0 & 0.0 & 0.1 & 0.0\end{array}$

$\begin{array}{llllllllllllllll}15 & 242.844 & 33.487 & 242.934 & 33.420 & 11.191 & 15.0 & 131.6 & 90 & 4.1 & 0.5 & 0.0 & 0.0 & -0.0 & 0.0\end{array}$

$\begin{array}{lllllllllllllll}16 & 242.934 & 33.420 & 242.969 & 33.386 & 4.982 & 15.0 & 139.2 & 90 & 4.1 & 0.5 & 0.0 & 0.0 & 0.5 & 0.1\end{array}$

$\begin{array}{llllllllllllllll}17 & 242.969 & 33.386 & 242.998 & 33.357 & 4.199 & 15.0 & 140.0 & 90 & 4.1 & 0.5 & 0.0 & 0.0 & 0.5 & 0.1\end{array}$

$\begin{array}{lllllllllllllllll}18 & 242.998 & 33.357 & 243.048 & 33.334 & 5.308 & 15.0 & 118.7 & 90 & 4.0 & 0.5 & 0.0 & 0.0 & -1.0 & 0.1\end{array}$

$\begin{array}{llllllllllllllll}19 & 243.048 & 33.334 & 243.156 & 33.281 & 11.650 & 15.0 & 120.3 & 90 & 4.0 & 0.5 & 0.0 & 0.0 & -0.9 & 0.1\end{array}$

$\begin{array}{llllllllllllllll}20 & 243.156 & 33.281 & 243.209 & 33.248 & 6.147 & 15.0 & 126.5 & 90 & 4.1 & 0.5 & 0.0 & 0.0 & -0.5 & 0.1\end{array}$

$\begin{array}{llllllllllllllll}21 & 243.209 & 33.248 & 243.268 & 33.204 & 7.353 & 15.0 & 131.6 & 90 & 4.1 & 0.5 & 0.0 & 0.0 & -0.1 & 0.0\end{array}$

$\begin{array}{llllllllllllllll}22 & 243.268 & 33.204 & 243.310 & 33.169 & 5.514 & 15.0 & 134.7 & 90 & 4.1 & 0.5 & 0.0 & 0.0 & 0.1 & 0.0\end{array}$

$\begin{array}{lllllllllllllllll}23 & 243.310 & 33.169 & 243.372 & 33.124 & 7.640 & 15.0 & 130.8 & 90 & 4.1 & 0.5 & 0.0 & 0.0 & -0.2 & 0.0\end{array}$

$\begin{array}{llllllllllllllll}24 & 243.372 & 33.124 & 243.447 & 33.071 & 9.142 & 15.0 & 130.0 & 90 & 4.1 & 0.5 & 0.0 & 0.0 & -0.3 & 0.0\end{array}$

$\begin{array}{lllllllllllllll}25 & 243.447 & 33.071 & 243.496 & 33.026 & 6.772 & 15.0 & 137.5 & 90 & 4.1 & 0.5 & 0.0 & 0.0 & 0.2 & 0.0\end{array}$ 
\# Lon-1 Lat-1 Lon-2 Lat-2 L(km) $\mathrm{z}(\mathrm{km})$ strike dip U_S S_S U_d S_d U_t S_t

$\begin{array}{llllllllllllllll}26 & 243.496 & 33.026 & 243.544 & 32.992 & 5.860 & 15.0 & 130.0 & 90 & 4.1 & 0.5 & 0.0 & 0.0 & -0.4 & 0.0\end{array}$ $\begin{array}{llllllllllllllll}27 & 243.544 & 32.992 & 243.556 & 32.988 & 1.206 & 15.0 & 111.6 & 90 & 3.8 & 0.4 & 0.0 & 0.0 & -1.7 & 0.2\end{array}$ $\begin{array}{lllllllllllllll}28 & 243.556 & 32.988 & 243.687 & 32.969 & 12.425 & 15.0 & 99.7 & 90 & 3.4 & 0.4 & 0.0 & 0.0 & -2.4 & 0.3\end{array}$ $\begin{array}{lllllllllllllllll}29 & 243.687 & 32.969 & 243.755 & 32.891 & 10.737 & 15.0 & 143.7 & 90 & 4.1 & 0.5 & 0.0 & 0.0 & 0.6 & 0.1\end{array}$ $\begin{array}{lllllllllllllll}30 & 243.755 & 32.891 & 243.822 & 32.839 & 8.519 & 15.0 & 132.6 & 90 & 4.1 & 0.5 & 0.0 & 0.0 & -0.3 & 0.0\end{array}$ $\begin{array}{llllllllllllllll}31 & 243.822 & 32.839 & 243.901 & 32.816 & 7.825 & 15.0 & 109.0 & 90 & 3.7 & 0.4 & 0.0 & 0.0 & -1.9 & 0.2\end{array}$ $\begin{array}{llllllllllllllll}32 & 243.901 & 32.816 & 243.959 & 32.787 & 6.313 & 15.0 & 120.6 & 90 & 4.0 & 0.5 & 0.0 & 0.0 & -1.2 & 0.1\end{array}$ $\begin{array}{llllllllllllllll}33 & 243.959 & 32.787 & 244.106 & 32.653 & 20.267 & 15.0 & 137.1 & 90 & 4.2 & 0.5 & 0.0 & 0.0 & -0.0 & 0.0\end{array}$

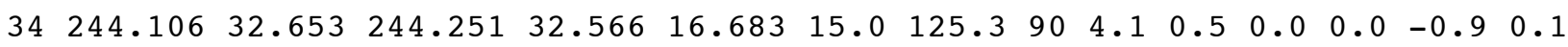
$\begin{array}{lllllllllllllll}35 & 244.251 & 32.566 & 244.435 & 32.391 & 25.995 & 15.0 & 138.2 & 90 & 4.2 & 0.5 & 0.0 & 0.0 & -0.0 & 0.0\end{array}$ $\begin{array}{lllllllllllllll}36 & 244.435 & 32.391 & 244.546 & 32.315 & 13.424 & 15.0 & 128.9 & 90 & 4.1 & 0.5 & 0.0 & 0.0 & -0.8 & 0.1\end{array}$ $\begin{array}{lllllllllllllll}37 & 244.546 & 32.315 & 245.007 & 32.136 & 47.773 & 15.0 & 114.4 & 90 & 3.8 & 0.4 & 0.0 & 0.0 & -1.8 & 0.2\end{array}$

\section{Whittier}

$\begin{array}{lllllllllllllll}1 & 241.862 & 34.118 & 241.883 & 34.086 & 4.044 & 15.0 & 151.4 & 90 & 11.0 & 1.8 & 0.0 & 0.0 & 0.0 & 0.0\end{array}$

$\begin{array}{llllllllllllllll}2 & 241.883 & 34.086 & 241.931 & 34.028 & 7.812 & 15.0 & 145.4 & 90 & 11.0 & 1.8 & 0.0 & 0.0 & 0.5 & 0.3\end{array}$

$\begin{array}{llllllllllllllll}3 & 241.931 & 34.028 & 241.952 & 33.997 & 3.948 & 15.0 & 150.6 & 90 & 10.8 & 1.8 & 0.0 & 0.0 & 3.1 & 0.8\end{array}$

$\begin{array}{lllllllllllllll}4 & 241.952 & 33.997 & 242.008 & 33.973 & 5.819 & 15.0 & 117.2 & 111 & 11.1 & 1.8 & 5.9 & 2.7 & 0.0 & 0.0\end{array}$

$\begin{array}{llllllllllllllll}5 & 242.008 & 33.973 & 242.148 & 33.931 & 13.754 & 15.0 & 109.8 & 90 & 10.8 & 1.8 & 0.0 & 0.0 & -0.8 & 1.4\end{array}$

$\begin{array}{lllllllllllllllll}6 & 242.148 & 33.931 & 242.208 & 33.909 & 6.061 & 15.0 & 113.7 & 90 & 4.2 & 1.6 & 0.0 & 0.0 & -2.7 & 1.1\end{array}$

$\begin{array}{llllllllllllllll}7 & 242.208 & 33.909 & 242.283 & 33.875 & 7.896 & 15.0 & 118.5 & 90 & 4.4 & 1.6 & 0.0 & 0.0 & -1.1 & 1.2\end{array}$

$\begin{array}{llllllllllllllll}8 & 242.283 & 33.875 & 242.364 & 33.853 & 7.882 & 15.0 & 108.0 & 90 & 4.2 & 1.5 & 0.0 & 0.0 & -0.6 & 1.5\end{array}$

$\begin{array}{llllllllllllllll}9 & 242.364 & 33.853 & 242.410 & 33.831 & 4.907 & 15.0 & 119.8 & 90 & 4.1 & 1.6 & 0.0 & 0.0 & 1.4 & 1.7\end{array}$

$\begin{array}{lllllllllllllll}10 & 242.410 & 33.831 & 242.452 & 33.815 & 4.274 & 15.0 & 114.5 & 90 & 4.3 & 1.6 & 0.0 & 0.0 & 1.8 & 1.9\end{array}$

\section{Puente Hills}

$\begin{array}{llllllllllllllll}1 & 241.611 & 34.074 & 241.664 & 34.034 & 6.605 & 15.0 & 132.2 & 90 & -6.8 & 1.6 & 0.0 & 0.0 & -2.2 & 1.5\end{array}$

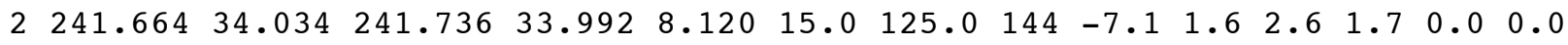

$\begin{array}{llllllllllllllll}3 & 241.736 & 33.992 & 241.798 & 33.960 & 6.740 & 15.0 & 121.8 & 145 & -7.2 & 1.6 & 3.1 & 1.6 & 0.0 & 0.0\end{array}$

$\begin{array}{lllllllllllllll}4 & 241.798 & 33.960 & 241.956 & 33.895 & 16.291 & 15.0 & 116.2 & 90 & -7.4 & 1.6 & 0.0 & 0.0 & -3.0 & 1.3\end{array}$

$\begin{array}{llllllllllllllll}5 & 241.956 & 33.895 & 242.033 & 33.876 & 7.428 & 15.0 & 106.5 & 147 & -8.0 & 1.7 & 3.5 & 1.8 & 0.0 & 0.0\end{array}$

$\begin{array}{llllllllllllllll}6 & 242.033 & 33.876 & 242.084 & 33.892 & 5.041 & 15.0 & 69.4 & 148 & -8.4 & 2.0 & -2.3 & 1.5 & 0.0 & 0.0\end{array}$

$\begin{array}{llllllllllllllll}7 & 242.084 & 33.892 & 242.148 & 33.931 & 7.331 & 15.0 & 53.8 & 90 & -7.6 & 2.0 & 0.0 & 0.0 & 3.5 & 1.3\end{array}$

\section{Raymond Hills}

$\begin{array}{llllllllllllllll}1 & 241.611 & 34.074 & 241.716 & 34.111 & 10.523 & 15.0 & 67.0 & 134 & 2.6 & 1.4 & 7.5 & 2.1 & 0.0 & 0.0\end{array}$

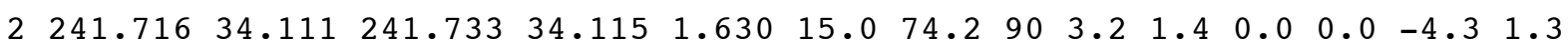

$\begin{array}{lllllllllllllll}3 & 241.733 & 34.115 & 241.778 & 34.123 & 4.245 & 15.0 & 77.9 & 90 & 3.5 & 1.5 & 0.0 & 0.0 & -3.9 & 1.3\end{array}$

$\begin{array}{llllllllllllllll}4 & 241.778 & 34.123 & 241.806 & 34.132 & 2.769 & 15.0 & 68.9 & 90 & 2.9 & 1.4 & 0.0 & 0.0 & -4.1 & 1.3\end{array}$

$\begin{array}{llllllllllllllll}5 & 241.806 & 34.132 & 241.838 & 34.128 & 2.985 & 15.0 & 98.5 & 90 & 4.5 & 1.5 & 0.0 & 0.0 & -1.9 & 1.2\end{array}$

$\begin{array}{llllllllllllllll}6 & 241.838 & 34.128 & 241.861 & 34.119 & 2.345 & 15.0 & 115.2 & 90 & 4.8 & 1.5 & 0.0 & 0.0 & -0.4 & 1.2\end{array}$

$\begin{array}{lllllllllllllll}7 & 241.861 & 34.119 & 241.877 & 34.120 & 1.480 & 15.0 & 85.7 & 90 & 0.1 & 1.4 & 0.0 & 0.0 & 7.6 & 2.0\end{array}$

$\begin{array}{lllllllllllllll}8 & 241.877 & 34.120 & 241.947 & 34.140 & 6.828 & 15.0 & 71.0 & 90 & 2.0 & 1.4 & 0.0 & 0.0 & 6.5 & 1.9\end{array}$

$\begin{array}{lllllllllllllll}9 & 241.947 & 34.140 & 242.025 & 34.164 & 7.670 & 15.0 & 69.7 & 90 & 2.1 & 1.4 & 0.0 & 0.0 & 5.0 & 1.8\end{array}$

\section{Santa Monica}

$\begin{array}{lllllllllllllll}1 & 240.485 & 33.989 & 240.653 & 33.980 & 15.556 & 15.0 & 93.6 & 90 & -1.7 & 1.0 & 0.0 & 0.0 & 7.9 & 2.3\end{array}$

$\begin{array}{llllllllllllllll}2 & 240.653 & 33.980 & 240.740 & 33.985 & 8.058 & 15.0 & 86.0 & 90 & -0.7 & 1.0 & 0.0 & 0.0 & 7.4 & 2.1\end{array}$

$\begin{array}{llllllllllllllll}3 & 240.740 & 33.985 & 240.772 & 33.941 & 5.707 & 15.0 & 148.8 & 90 & -6.7 & 1.8 & 0.0 & 0.0 & 2.5 & 1.3\end{array}$

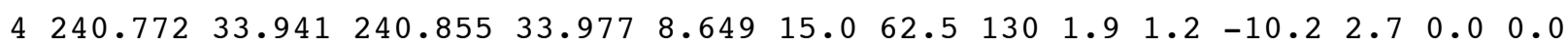

$\begin{array}{llllllllllllllll}5 & 240.855 & 33.977 & 240.980 & 33.972 & 11.565 & 15.0 & 92.7 & 135 & -1.5 & 1.0 & -8.7 & 2.4 & 0.0 & 0.0\end{array}$

$\begin{array}{lllllllllllllll}6 & 240.980 & 33.972 & 241.065 & 33.949 & 8.260 & 15.0 & 108.0 & 132 & -3.0 & 1.1 & -7.4 & 2.2 & 0.0 & 0.0\end{array}$

$\begin{array}{llllllllllllllll}7 & 241.065 & 33.949 & 241.181 & 33.960 & 10.792 & 15.0 & 83.5 & 134 & -0.8 & 1.0 & -7.5 & 2.1 & 0.0 & 0.0\end{array}$

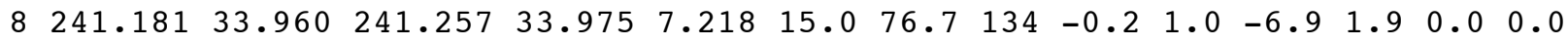

$\begin{array}{lllllllllllllll}9 & 241.257 & 33.975 & 241.305 & 33.985 & 4.572 & 15.0 & 75.9 & 134 & -0.1 & 1.0 & -6.5 & 1.9 & 0.0 & 0.0\end{array}$

$\begin{array}{lllllllllllllll}10 & 241.305 & 33.985 & 241.448 & 34.021 & 13.801 & 15.0 & 73.1 & 139 & 0.1 & 1.0 & -5.3 & 1.7 & 0.0 & 0.0\end{array}$

$\begin{array}{lllllllllllllll}11 & 241.448 & 34.021 & 241.459 & 34.032 & 1.588 & 15.0 & 39.8 & 90 & 1.6 & 0.9 & 0.0 & 0.0 & 1.4 & 1.1\end{array}$

$\begin{array}{llllllllllllllll}12 & 241.459 & 34.032 & 241.525 & 34.036 & 6.111 & 15.0 & 85.8 & 116 & 0.2 & 1.0 & -4.4 & 2.2 & 0.0 & 0.0\end{array}$ 
\# Lon-1 Lat-1 Lon-2 Lat-2 L(km) $\mathrm{z}(\mathrm{km})$ strike dip U_S S_S U_d S_d U_t S_t

$\begin{array}{lllllllllllllllll}13 & 241.525 & 34.036 & 241.566 & 34.052 & 4.181 & 15.0 & 64.9 & 90 & 0.8 & 0.9 & 0.0 & 0.0 & 1.5 & 1.0\end{array}$ $\begin{array}{llllllllllllllll}14 & 241.566 & 34.052 & 241.611 & 34.074 & 4.818 & 15.0 & 59.6 & 136 & 0.9 & 0.9 & -1.7 & 1.4 & 0.0 & 0.0\end{array}$

\section{Malibu}

$\begin{array}{llllllllllllllll}1 & 239.489 & 34.022 & 239.569 & 33.991 & 8.151 & 15.0 & 114.9 & 90 & 1.7 & 0.7 & 0.0 & 0.0 & -1.0 & 0.9\end{array}$

$\begin{array}{lllllllllllllll}2 & 239.569 & 33.991 & 239.642 & 33.989 & 6.749 & 15.0 & 91.9 & 90 & 1.1 & 0.5 & 0.0 & 0.0 & -1.8 & 1.0\end{array}$

$\begin{array}{llllllllllllllll}3 & 239.642 & 33.989 & 239.700 & 33.986 & 5.369 & 15.0 & 93.5 & 90 & 1.2 & 0.5 & 0.0 & 0.0 & -2.0 & 1.0\end{array}$

$\begin{array}{llllllllllllllll}4 & 239.700 & 33.986 & 239.770 & 33.985 & 6.469 & 15.0 & 91.0 & 90 & 1.1 & 0.5 & 0.0 & 0.0 & -2.2 & 1.0\end{array}$

$\begin{array}{lllllllllllllll}5 & 239.770 & 33.985 & 239.820 & 33.974 & 4.779 & 15.0 & 104.8 & 90 & 1.6 & 0.6 & 0.0 & 0.0 & -2.1 & 0.9\end{array}$

$\begin{array}{llllllllllllllll}6 & 239.820 & 33.974 & 239.889 & 33.973 & 6.377 & 15.0 & 91.0 & 90 & 1.0 & 0.5 & 0.0 & 0.0 & -2.6 & 0.9\end{array}$

$\begin{array}{llllllllllllllll}7 & 239.889 & 33.973 & 240.034 & 33.987 & 13.489 & 15.0 & 83.3 & 90 & 0.6 & 0.5 & 0.0 & 0.0 & -3.1 & 0.9\end{array}$

$\begin{array}{lllllllllllllll}8 & 240.034 & 33.987 & 240.094 & 34.013 & 6.248 & 15.0 & 62.5 & 90 & -0.6 & 0.5 & 0.0 & 0.0 & -3.5 & 0.8\end{array}$

$\begin{array}{lllllllllllllll}9 & 240.094 & 34.013 & 240.126 & 34.033 & 3.695 & 15.0 & 53.1 & 90 & -1.2 & 0.5 & 0.0 & 0.0 & -3.5 & 0.7\end{array}$

$\begin{array}{llllllllllllllll}10 & 240.126 & 34.033 & 240.222 & 34.017 & 9.042 & 15.0 & 101.3 & 90 & 1.9 & 0.5 & 0.0 & 0.0 & -3.4 & 0.7\end{array}$

$\begin{array}{llllllllllllllll}11 & 240.222 & 34.017 & 240.275 & 34.007 & 5.020 & 15.0 & 102.8 & 90 & 2.0 & 0.5 & 0.0 & 0.0 & -3.6 & 0.6\end{array}$

$\begin{array}{llllllllllllllll}12 & 240.275 & 34.007 & 240.388 & 33.987 & 10.673 & 15.0 & 102.0 & 90 & 1.9 & 0.5 & 0.0 & 0.0 & -3.9 & 0.6\end{array}$

$\begin{array}{lllllllllllllllll}13 & 240.388 & 33.987 & 240.485 & 33.989 & 8.965 & 15.0 & 88.6 & 90 & 0.9 & 0.5 & 0.0 & 0.0 & -4.6 & 0.6\end{array}$

\section{Oak Ridge}

$\begin{array}{lllllllllllllll}1 & 239.467 & 34.095 & 239.880 & 34.251 & 41.825 & 15.0 & 65.4 & 90 & -4.8 & 0.6 & 0.0 & 0.0 & -7.1 & 1.1\end{array}$

$\begin{array}{lllllllllllllll}2 & 239.880 & 34.251 & 239.939 & 34.277 & 6.152 & 15.0 & 62.0 & 161 & -5.2 & 0.6 & 6.3 & 1.0 & 0.0 & 0.0\end{array}$

$\begin{array}{llllllllllllllll}3 & 239.939 & 34.277 & 240.021 & 34.306 & 8.206 & 15.0 & 66.9 & 159 & -4.7 & 0.6 & 6.5 & 0.9 & 0.0 & 0.0\end{array}$

$\begin{array}{lllllllllllllll}4 & 240.021 & 34.306 & 240.035 & 34.318 & 1.853 & 15.0 & 44.1 & 90 & -6.6 & 0.7 & 0.0 & 0.0 & -3.6 & 0.8\end{array}$

$\begin{array}{lllllllllllllll}5 & 240.035 & 34.318 & 240.134 & 34.314 & 9.123 & 15.0 & 92.8 & 90 & -1.7 & 0.6 & 0.0 & 0.0 & -7.1 & 0.8\end{array}$

$\begin{array}{llllllllllllllll}6 & 240.134 & 34.314 & 240.207 & 34.296 & 7.010 & 15.0 & 106.5 & 90 & 0.0 & 0.6 & 0.0 & 0.0 & -7.0 & 0.8\end{array}$

$\begin{array}{lllllllllllllll}7 & 240.207 & 34.296 & 240.259 & 34.264 & 5.960 & 15.0 & 126.5 & 90 & 2.4 & 0.7 & 0.0 & 0.0 & -6.4 & 0.7\end{array}$

$\begin{array}{llllllllllllllll}8 & 240.259 & 34.264 & 240.322 & 34.254 & 5.908 & 15.0 & 100.8 & 33 & -0.6 & 0.6 & 7.8 & 0.8 & 0.0 & 0.0\end{array}$

$\begin{array}{lllllllllllllllll}9 & 240.322 & 34.254 & 240.394 & 34.263 & 6.706 & 15.0 & 81.4 & 33 & -2.7 & 0.6 & 6.9 & 0.8 & 0.0 & 0.0\end{array}$

$\begin{array}{llllllllllllllll}10 & 240.394 & 34.263 & 240.485 & 34.252 & 8.470 & 15.0 & 98.3 & 33 & -0.9 & 0.6 & 7.2 & 0.8 & 0.0 & 0.0\end{array}$

$\begin{array}{llllllllllllllll}11 & 240.485 & 34.252 & 240.568 & 34.236 & 7.849 & 15.0 & 103.0 & 90 & -0.5 & 0.6 & 0.0 & 0.0 & -5.8 & 0.6\end{array}$

$\begin{array}{lllllllllllllll}12 & 240.568 & 34.236 & 240.649 & 34.233 & 7.470 & 15.0 & 92.5 & 32 & -1.5 & 0.6 & 6.3 & 0.7 & 0.0 & 0.0\end{array}$

$\begin{array}{lllllllllllllll}13 & 240.649 & 34.233 & 240.727 & 34.253 & 7.520 & 15.0 & 72.8 & 34 & -3.2 & 0.6 & 5.2 & 0.7 & 0.0 & 0.0\end{array}$

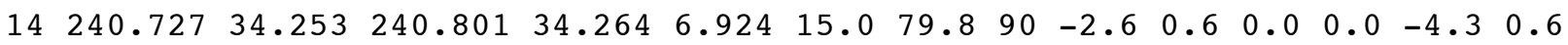

$\begin{array}{llllllllllllllll}15 & 240.801 & 34.264 & 240.907 & 34.317 & 11.393 & 15.0 & 58.9 & 90 & -4.0 & 0.6 & 0.0 & 0.0 & -2.8 & 0.6\end{array}$

$\begin{array}{lllllllllllllll}16 & 240.907 & 34.317 & 240.957 & 34.351 & 5.949 & 15.0 & 50.6 & 90 & -4.3 & 0.6 & 0.0 & 0.0 & -1.9 & 0.7\end{array}$

$\begin{array}{lllllllllllllll}17 & 240.957 & 34.351 & 241.015 & 34.347 & 5.355 & 15.0 & 94.7 & 68 & -1.9 & 0.6 & 11.1 & 1.8 & 0.0 & 0.0\end{array}$

$\begin{array}{lllllllllllllll}18 & 241.015 & 34.347 & 241.095 & 34.383 & 8.373 & 15.0 & 61.5 & 90 & -3.8 & 0.6 & 0.0 & 0.0 & -2.2 & 0.7\end{array}$

$\begin{array}{lllllllllllllll}19 & 241.095 & 34.383 & 241.229 & 34.372 & 12.385 & 15.0 & 95.6 & 90 & -2.0 & 0.6 & 0.0 & 0.0 & -3.6 & 0.7\end{array}$

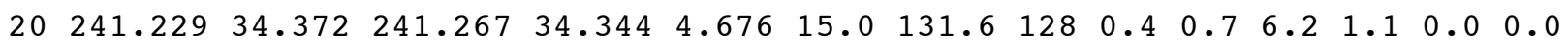

$\begin{array}{lllllllllllllll}21 & 241.267 & 34.344 & 241.319 & 34.355 & 4.937 & 15.0 & 75.7 & 133 & -2.9 & 0.6 & 3.3 & 1.2 & 0.0 & 0.0\end{array}$

$\begin{array}{llllllllllllllll}22 & 241.319 & 34.355 & 241.388 & 34.310 & 8.077 & 15.0 & 128.2 & 121 & 0.0 & 0.7 & 6.7 & 1.4 & 0.0 & 0.0\end{array}$

$\begin{array}{llllllllllllllll}23 & 241.388 & 34.310 & 241.453 & 34.302 & 6.049 & 15.0 & 98.4 & 126 & -1.7 & 0.6 & 4.7 & 1.5 & 0.0 & 0.0\end{array}$

$\begin{array}{llllllllllllllll}24 & 241.453 & 34.302 & 241.511 & 34.328 & 6.068 & 15.0 & 61.6 & 124 & -2.9 & 0.7 & 1.8 & 1.6 & 0.0 & 0.0\end{array}$

$\begin{array}{lllllllllllllll}25 & 241.511 & 34.328 & 241.562 & 34.335 & 4.757 & 15.0 & 80.6 & 90 & -2.5 & 0.6 & 0.0 & 0.0 & -1.7 & 1.0\end{array}$

\section{Santa Ynez}

$\begin{array}{llllllllllllllll}1 & 239.357 & 34.460 & 239.693 & 34.514 & 31.439 & 15.0 & 78.9 & 90 & -1.8 & 0.6 & 0.0 & 0.0 & 3.8 & 0.8\end{array}$

$\begin{array}{lllllllllllllll}2 & 239.693 & 34.514 & 239.737 & 34.516 & 4.046 & 15.0 & 86.8 & 90 & -2.2 & 0.5 & 0.0 & 0.0 & 3.1 & 0.7\end{array}$

$\begin{array}{lllllllllllllll}3 & 239.737 & 34.516 & 239.792 & 34.526 & 5.170 & 15.0 & 77.6 & 90 & -1.7 & 0.6 & 0.0 & 0.0 & 3.4 & 0.6\end{array}$

$\begin{array}{lllllllllllllll}4 & 239.792 & 34.526 & 239.880 & 34.546 & 8.378 & 15.0 & 74.6 & 90 & -1.5 & 0.6 & 0.0 & 0.0 & 3.3 & 0.6\end{array}$

$\begin{array}{llllllllllllllll}5 & 239.880 & 34.546 & 239.969 & 34.564 & 8.409 & 15.0 & 76.2 & 90 & -1.6 & 0.6 & 0.0 & 0.0 & 3.1 & 0.5\end{array}$

$\begin{array}{llllllllllllllll}6 & 239.969 & 34.564 & 240.023 & 34.562 & 4.961 & 15.0 & 92.5 & 90 & -2.4 & 0.5 & 0.0 & 0.0 & 2.4 & 0.5\end{array}$

$\begin{array}{llllllllllllllll}7 & 240.023 & 34.562 & 240.065 & 34.549 & 4.116 & 15.0 & 110.5 & 90 & -3.0 & 0.5 & 0.0 & 0.0 & 1.4 & 0.6\end{array}$

$\begin{array}{lllllllllllllll}8 & 240.065 & 34.549 & 240.133 & 34.555 & 6.277 & 15.0 & 83.9 & 90 & -2.1 & 0.5 & 0.0 & 0.0 & 2.5 & 0.5\end{array}$

$\begin{array}{llllllllllllllll}9 & 240.133 & 34.555 & 240.194 & 34.550 & 5.626 & 15.0 & 95.6 & 70 & -2.5 & 0.5 & -5.6 & 1.4 & 0.0 & 0.0\end{array}$ $\begin{array}{llllllllllllllll}10 & 240.194 & 34.550 & 240.268 & 34.522 & 7.470 & 15.0 & 114.6 & 90 & -3.0 & 0.5 & 0.0 & 0.0 & 0.9 & 0.5\end{array}$ $\begin{array}{lllllllllllllll}11 & 240.268 & 34.522 & 240.364 & 34.501 & 9.118 & 15.0 & 104.8 & 70 & -2.8 & 0.5 & -3.5 & 1.3 & 0.0 & 0.0\end{array}$ $\begin{array}{lllllllllllllll}12 & 240.364 & 34.501 & 240.447 & 34.490 & 7.720 & 15.0 & 99.1 & 69 & -2.7 & 0.5 & -3.7 & 1.2 & 0.0 & 0.0\end{array}$ 
\# Lon-1 Lat-1 Lon-2 Lat-2 L(km) $\mathrm{z}(\mathrm{km})$ strike dip U_S S_S U_d S_d U_t S_t

$\begin{array}{llllllllllllllll}13 & 240.447 & 34.490 & 240.499 & 34.491 & 4.778 & 15.0 & 88.7 & 69 & -2.4 & 0.5 & -4.7 & 1.2 & 0.0 & 0.0\end{array}$ $\begin{array}{lllllllllllllll}14 & 240.499 & 34.491 & 240.565 & 34.500 & 6.143 & 15.0 & 80.6 & 90 & -2.2 & 0.5 & 0.0 & 0.0 & 1.9 & 0.4\end{array}$ $\begin{array}{lllllllllllllll}15 & 240.565 & 34.500 & 240.680 & 34.508 & 10.598 & 15.0 & 85.2 & 90 & -2.3 & 0.5 & 0.0 & 0.0 & 1.5 & 0.4\end{array}$ $\begin{array}{lllllllllllllll}16 & 240.680 & 34.508 & 240.770 & 34.531 & 8.649 & 15.0 & 72.8 & 90 & -1.9 & 0.5 & 0.0 & 0.0 & 1.8 & 0.4\end{array}$ $\begin{array}{llllllllllllllll}17 & 240.770 & 34.531 & 240.836 & 34.533 & 6.063 & 15.0 & 87.9 & 90 & -2.3 & 0.5 & 0.0 & 0.0 & 1.1 & 0.5\end{array}$ $\begin{array}{llllllllllllllll}18 & 240.836 & 34.533 & 241.028 & 34.582 & 18.440 & 15.0 & 72.8 & 90 & -2.0 & 0.5 & 0.0 & 0.0 & 1.4 & 0.5\end{array}$ $\begin{array}{lllllllllllllll}19 & 241.028 & 34.582 & 241.109 & 34.618 & 8.435 & 15.0 & 61.7 & 137 & -1.7 & 0.6 & -2.0 & 0.8 & 0.0 & 0.0\end{array}$ $\begin{array}{llllllllllllllll}20 & 241.109 & 34.618 & 241.157 & 34.623 & 4.437 & 15.0 & 82.8 & 131 & -2.1 & 0.5 & -1.0 & 1.0 & 0.0 & 0.0\end{array}$ $\begin{array}{lllllllllllllll}21 & 241.157 & 34.623 & 241.210 & 34.621 & 4.865 & 15.0 & 92.6 & 129 & -2.1 & 0.5 & -0.3 & 1.1 & 0.0 & 0.0\end{array}$

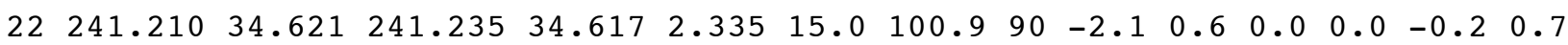

\section{Hosgri}

$\begin{array}{lllllllllllllllll}1 & 238.998 & 35.356 & 239.040 & 35.273 & 9.969 & 15.0 & 157.5 & 90 & 5.7 & 0.3 & 0.0 & 0.0 & -2.7 & 0.3\end{array}$ $\begin{array}{lllllllllllllll}2 & 239.040 & 35.273 & 239.084 & 35.194 & 9.636 & 15.0 & 155.4 & 90 & 5.6 & 0.3 & 0.0 & 0.0 & -3.1 & 0.3\end{array}$ $\begin{array}{llllllllllllllll}3 & 239.084 & 35.194 & 239.121 & 35.145 & 6.396 & 15.0 & 148.2 & 90 & 5.1 & 0.3 & 0.0 & 0.0 & -3.9 & 0.3\end{array}$ $\begin{array}{lllllllllllllllll}4 & 239.121 & 35.145 & 239.134 & 35.110 & 4.060 & 15.0 & 163.0 & 90 & 6.0 & 0.3 & 0.0 & 0.0 & -2.6 & 0.3\end{array}$ $\begin{array}{lllllllllllllllll}5 & 239.134 & 35.110 & 239.166 & 35.049 & 7.370 & 15.0 & 156.7 & 90 & 5.7 & 0.3 & 0.0 & 0.0 & -3.3 & 0.3\end{array}$ $\begin{array}{llllllllllllllll}6 & 239.166 & 35.049 & 239.195 & 34.977 & 8.415 & 15.0 & 161.7 & 90 & 5.9 & 0.3 & 0.0 & 0.0 & -3.0 & 0.3\end{array}$ $\begin{array}{lllllllllllllllll}7 & 239.195 & 34.977 & 239.205 & 34.936 & 4.639 & 15.0 & 168.6 & 90 & 6.3 & 0.3 & 0.0 & 0.0 & -2.4 & 0.3\end{array}$ $\begin{array}{llllllllllllllll}8 & 239.205 & 34.936 & 239.234 & 34.895 & 5.264 & 15.0 & 149.8 & 90 & 5.1 & 0.3 & 0.0 & 0.0 & -4.3 & 0.3\end{array}$ $\begin{array}{llllllllllllllll}9 & 239.234 & 34.895 & 239.254 & 34.857 & 4.595 & 15.0 & 156.5 & 90 & 5.6 & 0.3 & 0.0 & 0.0 & -3.8 & 0.3\end{array}$ $\begin{array}{lllllllllllllllll}10 & 239.254 & 34.857 & 239.267 & 34.797 & 6.762 & 15.0 & 169.9 & 90 & 6.4 & 0.3 & 0.0 & 0.0 & -2.5 & 0.3\end{array}$ $\begin{array}{lllllllllllllll}11 & 239.267 & 34.797 & 239.308 & 34.709 & 10.459 & 15.0 & 159.0 & 90 & 5.8 & 0.3 & 0.0 & 0.0 & -3.8 & 0.4\end{array}$ $\begin{array}{lllllllllllllll}12 & 239.308 & 34.709 & 239.312 & 34.665 & 4.895 & 15.0 & 175.7 & 90 & 6.6 & 0.3 & 0.0 & 0.0 & -2.2 & 0.4\end{array}$ $\begin{array}{lllllllllllllll}13 & 239.312 & 34.665 & 239.313 & 34.606 & 6.546 & 15.0 & 179.2 & 90 & 6.8 & 0.3 & 0.0 & 0.0 & -1.9 & 0.4\end{array}$ $\begin{array}{lllllllllllllll}14 & 239.313 & 34.606 & 239.335 & 34.533 & 8.346 & 15.0 & 166.0 & 90 & 6.1 & 0.3 & 0.0 & 0.0 & -3.5 & 0.4\end{array}$ $\begin{array}{lllllllllllllllll}15 & 239.335 & 34.533 & 239.357 & 34.460 & 8.346 & 15.0 & 166.0 & 90 & 6.1 & 0.3 & 0.0 & 0.0 & -3.7 & 0.4\end{array}$ $\begin{array}{llllllllllllllll}16 & 239.357 & 34.460 & 239.379 & 34.387 & 8.347 & 15.0 & 166.0 & 90 & 10.3 & 0.9 & 0.0 & 0.0 & -2.2 & 0.4\end{array}$ $\begin{array}{llllllllllllllll}17 & 239.379 & 34.387 & 239.401 & 34.314 & 8.347 & 15.0 & 166.0 & 90 & 10.3 & 0.9 & 0.0 & 0.0 & -2.2 & 0.4\end{array}$ $\begin{array}{lllllllllllllll}18 & 239.401 & 34.314 & 239.423 & 34.241 & 8.347 & 15.0 & 165.9 & 90 & 10.3 & 0.9 & 0.0 & 0.0 & -2.2 & 0.3\end{array}$ $\begin{array}{llllllllllllllll}19 & 239.423 & 34.241 & 239.445 & 34.168 & 8.348 & 15.0 & 165.9 & 90 & 10.3 & 0.9 & 0.0 & 0.0 & -2.2 & 0.3\end{array}$ $\begin{array}{llllllllllllllll}20 & 239.445 & 34.168 & 239.467 & 34.095 & 8.348 & 15.0 & 165.9 & 90 & 10.3 & 0.9 & 0.0 & 0.0 & -2.2 & 0.3\end{array}$ $\begin{array}{lllllllllllllll}21 & 239.467 & 34.095 & 239.489 & 34.022 & 8.348 & 15.0 & 165.9 & 90 & 1.7 & 1.1 & 0.0 & 0.0 & 0.9 & 0.5\end{array}$

\section{Santa Cruz-Santa Catalina}

$\begin{array}{llllllllllllllll}1 & 240.485 & 33.989 & 240.519 & 33.956 & 4.824 & 15.0 & 139.3 & 90 & 11.4 & 1.9 & 0.0 & 0.0 & -6.8 & 1.7\end{array}$ $\begin{array}{llllllllllllllll}2 & 240.519 & 33.956 & 240.637 & 33.893 & 12.957 & 15.0 & 122.6 & 90 & 9.0 & 1.4 & 0.0 & 0.0 & -9.7 & 1.9\end{array}$ $\begin{array}{lllllllllllllll}3 & 240.637 & 33.893 & 240.721 & 33.844 & 9.484 & 15.0 & 124.9 & 90 & 9.4 & 1.5 & 0.0 & 0.0 & -9.1 & 1.7\end{array}$ $\begin{array}{lllllllllllllll}4 & 240.721 & 33.844 & 240.797 & 33.791 & 9.169 & 15.0 & 129.9 & 90 & 10.1 & 1.6 & 0.0 & 0.0 & -8.1 & 1.4\end{array}$ $\begin{array}{lllllllllllllllll}5 & 240.797 & 33.791 & 240.866 & 33.747 & 8.042 & 15.0 & 127.3 & 90 & 9.7 & 1.5 & 0.0 & 0.0 & -8.3 & 1.3\end{array}$ $\begin{array}{llllllllllllllll}6 & 240.866 & 33.747 & 240.908 & 33.716 & 5.194 & 15.0 & 131.4 & 90 & 10.3 & 1.6 & 0.0 & 0.0 & -7.5 & 1.1\end{array}$ $\begin{array}{llllllllllllllll}7 & 240.908 & 33.716 & 240.951 & 33.686 & 5.193 & 15.0 & 129.8 & 90 & 10.1 & 1.6 & 0.0 & 0.0 & -7.7 & 1.1\end{array}$ $\begin{array}{lllllllllllllll}8 & 240.951 & 33.686 & 240.990 & 33.652 & 5.225 & 15.0 & 136.2 & 90 & 10.8 & 1.6 & 0.0 & 0.0 & -6.4 & 0.9\end{array}$ $\begin{array}{lllllllllllllll}9 & 240.990 & 33.652 & 241.142 & 33.564 & 17.154 & 15.0 & 124.6 & 90 & 9.3 & 1.5 & 0.0 & 0.0 & -8.2 & 1.0\end{array}$ $\begin{array}{lllllllllllllll}10 & 241.142 & 33.564 & 241.152 & 33.528 & 4.099 & 15.0 & 166.9 & 90 & 12.3 & 1.5 & 0.0 & 0.0 & 0.3 & 0.9\end{array}$ $\begin{array}{lllllllllllllll}11 & 241.152 & 33.528 & 241.202 & 33.500 & 5.588 & 15.0 & 123.8 & 90 & 9.3 & 1.6 & 0.0 & 0.0 & -8.1 & 0.9\end{array}$ $\begin{array}{lllllllllllllllll}12 & 241.202 & 33.500 & 241.265 & 33.416 & 11.005 & 15.0 & 147.8 & 90 & 11.7 & 1.6 & 0.0 & 0.0 & -3.5 & 0.9\end{array}$ $\begin{array}{llllllllllllllll}13 & 241.265 & 33.416 & 241.354 & 33.360 & 10.351 & 15.0 & 126.8 & 90 & 9.8 & 1.6 & 0.0 & 0.0 & -7.2 & 1.0\end{array}$ $\begin{array}{lllllllllllllll}14 & 241.354 & 33.360 & 241.484 & 33.301 & 13.759 & 15.0 & 118.4 & 90 & 8.6 & 1.6 & 0.0 & 0.0 & -8.4 & 1.1\end{array}$ $\begin{array}{lllllllllllllll}15 & 241.484 & 33.301 & 241.659 & 33.249 & 17.293 & 15.0 & 109.4 & 90 & 7.2 & 1.7 & 0.0 & 0.0 & -9.3 & 1.2\end{array}$ $\begin{array}{lllllllllllllll}16 & 241.659 & 33.249 & 241.835 & 33.203 & 17.181 & 15.0 & 107.2 & 90 & 6.8 & 1.7 & 0.0 & 0.0 & -9.2 & 1.4\end{array}$ $\begin{array}{lllllllllllllll}17 & 241.835 & 33.203 & 241.907 & 33.191 & 6.844 & 15.0 & 101.2 & 90 & 5.8 & 1.8 & 0.0 & 0.0 & -9.7 & 1.5\end{array}$ $\begin{array}{llllllllllllllll}18 & 241.907 & 33.191 & 241.977 & 33.166 & 7.093 & 15.0 & 113.0 & 90 & 7.7 & 1.6 & 0.0 & 0.0 & -8.1 & 1.8\end{array}$ $\begin{array}{llllllllllllllll}19 & 241.977 & 33.166 & 242.042 & 33.114 & 8.369 & 15.0 & 133.5 & 90 & 10.0 & 1.3 & 0.0 & 0.0 & -4.8 & 2.2\end{array}$ $\begin{array}{lllllllllllllll}20 & 242.042 & 33.114 & 242.133 & 33.020 & 13.450 & 15.0 & 140.8 & 90 & 10.5 & 1.2 & 0.0 & 0.0 & -3.3 & 2.4\end{array}$ $\begin{array}{lllllllllllllll}21 & 242.133 & 33.020 & 242.144 & 33.003 & 2.147 & 15.0 & 151.4 & 90 & 10.9 & 1.2 & 0.0 & 0.0 & -1.1 & 2.6\end{array}$ $\begin{array}{llllllllllllllll}22 & 242.144 & 33.003 & 242.172 & 32.975 & 4.061 & 15.0 & 139.9 & 90 & 10.5 & 1.2 & 0.0 & 0.0 & -3.2 & 2.6\end{array}$ $\begin{array}{lllllllllllllllll}23 & 242.172 & 32.975 & 242.230 & 32.935 & 7.006 & 15.0 & 129.3 & 90 & 9.7 & 1.4 & 0.0 & 0.0 & -5.0 & 2.7\end{array}$ 
\# Lon-1 Lat-1 Lon-2 Lat-2 L(km) $\mathrm{z}(\mathrm{km})$ strike dip U_S S_S U_d S_d U_t S_t

$\begin{array}{llllllllllllllll}24 & 242.230 & 32.935 & 242.301 & 32.830 & 13.407 & 15.0 & 150.3 & 90 & 10.8 & 1.2 & 0.0 & 0.0 & -1.0 & 3.0\end{array}$ $\begin{array}{llllllllllllllll}25 & 242.301 & 32.830 & 242.369 & 32.736 & 12.217 & 15.0 & 148.6 & 90 & 10.8 & 1.2 & 0.0 & 0.0 & -1.0 & 3.3\end{array}$ $\begin{array}{lllllllllllllll}26 & 242.369 & 32.736 & 242.396 & 32.685 & 6.197 & 15.0 & 155.9 & 90 & 10.8 & 1.2 & 0.0 & 0.0 & 0.5 & 3.5\end{array}$ $\begin{array}{llllllllllllllll}27 & 242.396 & 32.685 & 242.455 & 32.604 & 10.552 & 15.0 & 148.3 & 90 & 10.8 & 1.2 & 0.0 & 0.0 & -0.7 & 3.7\end{array}$ $\begin{array}{llllllllllllllll}28 & 242.455 & 32.604 & 242.533 & 32.489 & 14.708 & 15.0 & 150.1 & 90 & 10.8 & 1.2 & 0.0 & 0.0 & -0.1 & 3.9\end{array}$ $\begin{array}{lllllllllllllll}29 & 242.533 & 32.489 & 242.556 & 32.455 & 4.346 & 15.0 & 150.2 & 90 & 10.8 & 1.2 & 0.0 & 0.0 & 0.1 & 4.1\end{array}$ $\begin{array}{llllllllllllllll}30 & 242.556 & 32.455 & 242.570 & 32.449 & 1.475 & 15.0 & 116.8 & 90 & 9.1 & 2.4 & 0.0 & 0.0 & -5.8 & 3.6\end{array}$ $\begin{array}{llllllllllllllll}31 & 242.570 & 32.449 & 242.595 & 32.415 & 4.443 & 15.0 & 148.0 & 90 & 10.8 & 1.2 & 0.0 & 0.0 & -0.2 & 4.3\end{array}$ $\begin{array}{lllllllllllllll}32 & 242.595 & 32.415 & 242.647 & 32.320 & 11.616 & 15.0 & 155.1 & 90 & 10.7 & 1.2 & 0.0 & 0.0 & 1.2 & 4.4\end{array}$ $\begin{array}{lllllllllllllll}33 & 242.647 & 32.320 & 242.670 & 32.265 & 6.472 & 15.0 & 160.4 & 90 & 10.6 & 1.4 & 0.0 & 0.0 & 2.4 & 4.6\end{array}$ $\begin{array}{llllllllllllllll}34 & 242.670 & 32.265 & 242.756 & 32.169 & 13.381 & 15.0 & 142.7 & 90 & 10.8 & 1.3 & 0.0 & 0.0 & -0.7 & 4.8\end{array}$ $\begin{array}{lllllllllllllll}35 & 242.756 & 32.169 & 242.785 & 32.142 & 4.056 & 15.0 & 137.6 & 90 & 10.7 & 1.5 & 0.0 & 0.0 & -1.5 & 5.0\end{array}$ $\begin{array}{lllllllllllllll}36 & 242.785 & 32.142 & 242.832 & 32.070 & 9.134 & 15.0 & 150.9 & 90 & 10.8 & 1.2 & 0.0 & 0.0 & 1.1 & 5.2\end{array}$ $\begin{array}{lllllllllllllll}37 & 242.832 & 32.070 & 242.889 & 32.023 & 7.493 & 15.0 & 134.1 & 90 & 10.7 & 1.8 & 0.0 & 0.0 & -1.9 & 5.2\end{array}$ $\begin{array}{lllllllllllllll}38 & 242.889 & 32.023 & 242.916 & 31.983 & 5.117 & 15.0 & 150.1 & 90 & 10.7 & 1.2 & 0.0 & 0.0 & 1.3 & 5.5\end{array}$ $\begin{array}{lllllllllllllll}39 & 242.916 & 31.983 & 242.965 & 31.873 & 13.048 & 15.0 & 159.2 & 90 & 10.4 & 1.5 & 0.0 & 0.0 & 3.1 & 5.6\end{array}$ $\begin{array}{lllllllllllllll}40 & 242.965 & 31.873 & 242.998 & 31.827 & 5.981 & 15.0 & 148.5 & 90 & 10.8 & 1.2 & 0.0 & 0.0 & 1.3 & 5.9\end{array}$ $\begin{array}{llllllllllllllll}41 & 242.998 & 31.827 & 243.034 & 31.800 & 4.537 & 15.0 & 131.3 & 90 & 10.7 & 2.1 & 0.0 & 0.0 & -1.8 & 5.8\end{array}$ $\begin{array}{lllllllllllllll}42 & 243.034 & 31.800 & 243.103 & 31.755 & 8.223 & 15.0 & 127.3 & 90 & 10.6 & 2.5 & 0.0 & 0.0 & -2.4 & 5.8\end{array}$ $\begin{array}{lllllllllllllll}43 & 243.103 & 31.755 & 243.156 & 31.711 & 7.002 & 15.0 & 134.2 & 90 & 10.8 & 1.9 & 0.0 & 0.0 & -1.0 & 6.1\end{array}$ $\begin{array}{lllllllllllllll}44 & 243.156 & 31.711 & 243.199 & 31.686 & 4.930 & 15.0 & 124.2 & 90 & 10.5 & 2.8 & 0.0 & 0.0 & -2.8 & 5.9\end{array}$ $\begin{array}{lllllllllllllll}45 & 243.199 & 31.686 & 243.226 & 31.655 & 4.286 & 15.0 & 143.3 & 90 & 10.8 & 1.2 & 0.0 & 0.0 & 0.9 & 6.5\end{array}$ $\begin{array}{llllllllllllllll}46 & 243.226 & 31.655 & 243.294 & 31.603 & 8.652 & 15.0 & 131.8 & 90 & 10.8 & 2.1 & 0.0 & 0.0 & -1.1 & 6.4\end{array}$ $\begin{array}{llllllllllllllll}47 & 243.294 & 31.603 & 243.361 & 31.581 & 6.811 & 15.0 & 111.0 & 90 & 9.7 & 4.1 & 0.0 & 0.0 & -4.7 & 5.6\end{array}$

\section{Palos Verdes}

$\begin{array}{llllllllllllllll}1 & 241.443 & 33.972 & 241.448 & 34.021 & 5.455 & 15.0 & 4.9 & 90 & -1.2 & 1.2 & 0.0 & 0.0 & -1.0 & 1.1\end{array}$ $\begin{array}{llllllllllllllll}2 & 241.443 & 33.972 & 241.504 & 33.904 & 9.418 & 15.0 & 143.2 & 90 & -1.6 & 1.6 & 0.0 & 0.0 & 0.0 & 0.0\end{array}$ $\begin{array}{llllllllllllllll}3 & 241.504 & 33.904 & 241.561 & 33.865 & 6.820 & 15.0 & 129.3 & 90 & -1.6 & 1.6 & 0.0 & 0.0 & 0.3 & 0.5\end{array}$ $\begin{array}{llllllllllllllll}4 & 241.561 & 33.865 & 241.600 & 33.819 & 6.250 & 15.0 & 144.7 & 90 & -1.6 & 1.6 & 0.0 & 0.0 & -0.2 & 0.4\end{array}$ $\begin{array}{llllllllllllllll}5 & 241.600 & 33.819 & 241.666 & 33.791 & 6.855 & 15.0 & 116.9 & 90 & -1.5 & 1.5 & 0.0 & 0.0 & 0.5 & 0.9\end{array}$ $\begin{array}{llllllllllllllll}6 & 241.666 & 33.791 & 241.746 & 33.749 & 8.753 & 15.0 & 122.1 & 90 & -1.5 & 1.5 & 0.0 & 0.0 & 0.3 & 1.0\end{array}$ $\begin{array}{llllllllllllllll}7 & 241.746 & 33.749 & 241.767 & 33.694 & 6.403 & 15.0 & 162.3 & 90 & -1.4 & 1.6 & 0.0 & 0.0 & -0.8 & 1.1\end{array}$ $\begin{array}{llllllllllllllll}8 & 241.767 & 33.694 & 241.854 & 33.585 & 14.536 & 15.0 & 146.2 & 90 & -1.5 & 1.7 & 0.0 & 0.0 & -0.5 & 1.3\end{array}$ $\begin{array}{llllllllllllllll}9 & 241.854 & 33.585 & 241.881 & 33.547 & 4.904 & 15.0 & 149.2 & 90 & -1.5 & 1.7 & 0.0 & 0.0 & -0.6 & 1.6\end{array}$ $\begin{array}{llllllllllllllll}10 & 241.881 & 33.547 & 241.920 & 33.483 & 7.970 & 15.0 & 152.9 & 90 & -1.5 & 1.7 & 0.0 & 0.0 & -0.8 & 1.8\end{array}$ $\begin{array}{llllllllllllllll}11 & 241.920 & 33.483 & 241.940 & 33.445 & 4.607 & 15.0 & 156.2 & 90 & -1.4 & 1.7 & 0.0 & 0.0 & -0.9 & 2.0\end{array}$ $\begin{array}{lllllllllllllllll}12 & 241.940 & 33.445 & 242.014 & 33.356 & 12.035 & 15.0 & 145.1 & 90 & -1.6 & 1.6 & 0.0 & 0.0 & -0.7 & 2.3\end{array}$ $\begin{array}{lllllllllllllll}13 & 242.014 & 33.356 & 242.057 & 33.320 & 5.654 & 15.0 & 134.9 & 90 & -1.7 & 1.7 & 0.0 & 0.0 & -0.5 & 2.5\end{array}$ $\begin{array}{llllllllllllllll}14 & 242.057 & 33.320 & 242.083 & 33.282 & 4.861 & 15.0 & 150.1 & 90 & -1.5 & 1.7 & 0.0 & 0.0 & -0.9 & 2.7\end{array}$ $\begin{array}{llllllllllllllll}15 & 242.083 & 33.282 & 242.162 & 33.208 & 11.026 & 15.0 & 138.1 & 90 & -1.7 & 1.7 & 0.0 & 0.0 & -0.7 & 2.9\end{array}$ $\begin{array}{llllllllllllllll}16 & 242.162 & 33.208 & 242.258 & 33.075 & 17.257 & 15.0 & 148.7 & 90 & -1.5 & 1.7 & 0.0 & 0.0 & -1.1 & 3.3\end{array}$ $\begin{array}{llllllllllllllll}17 & 242.258 & 33.075 & 242.313 & 33.029 & 7.240 & 15.0 & 134.8 & 90 & -1.7 & 1.7 & 0.0 & 0.0 & -0.8 & 3.7\end{array}$ $\begin{array}{llllllllllllllll}18 & 242.313 & 33.029 & 242.384 & 32.954 & 10.640 & 15.0 & 141.4 & 90 & -1.6 & 1.7 & 0.0 & 0.0 & -1.1 & 4.0\end{array}$ $\begin{array}{lllllllllllllll}19 & 242.384 & 32.954 & 242.426 & 32.927 & 4.939 & 15.0 & 127.3 & 90 & -1.8 & 2.0 & 0.0 & 0.0 & -0.7 & 4.1\end{array}$ $\begin{array}{lllllllllllllll}20 & 242.426 & 32.927 & 242.480 & 32.867 & 8.355 & 15.0 & 142.8 & 90 & -1.6 & 1.6 & 0.0 & 0.0 & -1.2 & 4.4\end{array}$ $\begin{array}{lllllllllllllll}21 & 242.480 & 32.867 & 242.493 & 32.810 & 6.437 & 15.0 & 169.1 & 90 & -0.9 & 2.5 & 0.0 & 0.0 & -1.9 & 4.2\end{array}$ $\begin{array}{lllllllllllllll}22 & 242.493 & 32.810 & 242.533 & 32.754 & 7.253 & 15.0 & 148.9 & 90 & -1.5 & 1.7 & 0.0 & 0.0 & -1.5 & 4.8\end{array}$ $\begin{array}{lllllllllllllll}23 & 242.533 & 32.754 & 242.567 & 32.725 & 4.528 & 15.0 & 135.3 & 90 & -1.8 & 1.8 & 0.0 & 0.0 & -1.2 & 4.9\end{array}$ $\begin{array}{llllllllllllllll}24 & 242.567 & 32.725 & 242.587 & 32.680 & 5.331 & 15.0 & 159.4 & 90 & -1.1 & 2.1 & 0.0 & 0.0 & -1.8 & 5.0\end{array}$ $\begin{array}{lllllllllllllll}25 & 242.587 & 32.680 & 242.656 & 32.626 & 8.819 & 15.0 & 132.8 & 90 & -1.9 & 1.9 & 0.0 & 0.0 & -1.2 & 5.2\end{array}$ $\begin{array}{lllllllllllllll}26 & 242.656 & 32.626 & 242.685 & 32.596 & 4.299 & 15.0 & 140.7 & 90 & -1.7 & 1.7 & 0.0 & 0.0 & -1.5 & 5.5\end{array}$ $\begin{array}{lllllllllllllll}27 & 242.685 & 32.596 & 242.702 & 32.554 & 4.924 & 15.0 & 161.1 & 90 & -1.0 & 2.3 & 0.0 & 0.0 & -2.0 & 5.4\end{array}$ $\begin{array}{lllllllllllllll}28 & 242.702 & 32.554 & 242.734 & 32.497 & 7.000 & 15.0 & 154.6 & 90 & -1.3 & 1.9 & 0.0 & 0.0 & -1.9 & 5.8\end{array}$ $\begin{array}{llllllllllllllll}29 & 242.734 & 32.497 & 242.950 & 32.194 & 39.274 & 15.0 & 148.8 & 90 & -1.5 & 1.7 & 0.0 & 0.0 & -2.0 & 6.5\end{array}$ $\begin{array}{lllllllllllllll}30 & 242.950 & 32.194 & 243.165 & 31.890 & 39.354 & 15.0 & 148.9 & 90 & -1.5 & 1.7 & 0.0 & 0.0 & -2.3 & 7.7\end{array}$ $\begin{array}{llllllllllllllll}31 & 243.165 & 31.890 & 243.361 & 31.581 & 38.973 & 15.0 & 151.5 & 90 & -1.4 & 1.8 & 0.0 & 0.0 & -2.7 & 8.8\end{array}$ 
\# Lon-1 Lat-1 Lon-2 Lat-2 L(km) $\mathrm{z}(\mathrm{km})$ strike dip U_S S_S U_d S_d U_t S_t

\section{Oceanside}

$\begin{array}{lllllllllllllll}1 & 241.611 & 34.074 & 241.616 & 34.040 & 3.800 & 15.0 & 173.0 & 90 & -2.2 & 1.1 & 0.0 & 0.0 & 2.1 & 1.0\end{array}$

$\begin{array}{lllllllllllllll}2 & 241.616 & 34.040 & 241.640 & 33.989 & 6.076 & 15.0 & 158.6 & 90 & -1.6 & 1.2 & 0.0 & 0.0 & 2.5 & 0.9\end{array}$

$\begin{array}{llllllllllllllll}3 & 241.640 & 33.989 & 241.671 & 33.948 & 5.375 & 15.0 & 147.8 & 90 & -1.1 & 1.2 & 0.0 & 0.0 & 2.6 & 0.8\end{array}$

$\begin{array}{lllllllllllllll}4 & 241.671 & 33.948 & 241.749 & 33.877 & 10.680 & 15.0 & 137.5 & 90 & -0.6 & 1.2 & 0.0 & 0.0 & 2.6 & 0.8\end{array}$

$\begin{array}{lllllllllllllll}5 & 241.749 & 33.877 & 241.794 & 33.827 & 6.935 & 15.0 & 143.1 & 90 & -0.9 & 1.2 & 0.0 & 0.0 & 2.3 & 0.8\end{array}$

$\begin{array}{lllllllllllllll}6 & 241.794 & 33.827 & 241.850 & 33.789 & 6.682 & 15.0 & 129.1 & 90 & -0.3 & 1.2 & 0.0 & 0.0 & 2.3 & 0.8\end{array}$

$\begin{array}{lllllllllllllll}7 & 241.850 & 33.789 & 241.937 & 33.718 & 11.269 & 15.0 & 134.3 & 90 & -0.5 & 1.2 & 0.0 & 0.0 & 2.0 & 0.8\end{array}$

$\begin{array}{lllllllllllllll}8 & 241.937 & 33.718 & 242.007 & 33.674 & 8.120 & 15.0 & 126.9 & 90 & -0.2 & 1.2 & 0.0 & 0.0 & 1.8 & 0.8\end{array}$

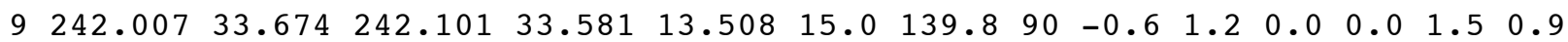

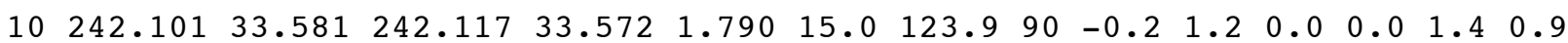

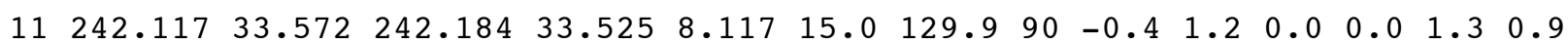

$\begin{array}{lllllllllllllll}12 & 242.184 & 33.525 & 242.209 & 33.503 & 3.369 & 15.0 & 136.4 & 90 & -0.5 & 1.2 & 0.0 & 0.0 & 1.1 & 1.0\end{array}$

$\begin{array}{llllllllllllllll}13 & 242.209 & 33.503 & 242.218 & 33.495 & 1.219 & 15.0 & 136.7 & 90 & -0.5 & 1.2 & 0.0 & 0.0 & 1.0 & 1.0\end{array}$

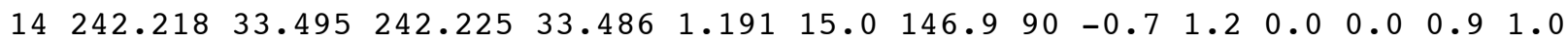

$\begin{array}{llllllllllllllll}15 & 242.225 & 33.486 & 242.236 & 33.473 & 1.768 & 15.0 & 144.7 & 90 & -0.7 & 1.2 & 0.0 & 0.0 & 0.9 & 1.0\end{array}$

$\begin{array}{lllllllllllllllll}16 & 242.236 & 33.473 & 242.246 & 33.460 & 1.716 & 15.0 & 147.2 & 90 & -0.7 & 1.2 & 0.0 & 0.0 & 0.8 & 1.0\end{array}$

$\begin{array}{llllllllllllllll}17 & 242.246 & 33.460 & 242.293 & 33.414 & 6.718 & 15.0 & 139.4 & 90 & -0.6 & 1.2 & 0.0 & 0.0 & 0.8 & 1.0\end{array}$

$\begin{array}{lllllllllllllll}18 & 242.293 & 33.414 & 242.390 & 33.310 & 14.648 & 15.0 & 141.9 & 90 & -0.6 & 1.2 & 0.0 & 0.0 & 0.5 & 1.1\end{array}$

$\begin{array}{llllllllllllllll}19 & 242.390 & 33.310 & 242.443 & 33.264 & 7.099 & 15.0 & 135.9 & 90 & -0.6 & 1.2 & 0.0 & 0.0 & 0.3 & 1.2\end{array}$

$\begin{array}{lllllllllllllll}20 & 242.443 & 33.264 & 242.513 & 33.222 & 8.016 & 15.0 & 125.5 & 90 & -0.5 & 1.2 & 0.0 & 0.0 & 0.2 & 1.2\end{array}$

$\begin{array}{lllllllllllllll}21 & 242.513 & 33.222 & 242.575 & 33.130 & 11.728 & 15.0 & 150.4 & 90 & -0.5 & 1.2 & 0.0 & 0.0 & -0.2 & 1.3\end{array}$

$\begin{array}{lllllllllllllll}22 & 242.575 & 33.130 & 242.624 & 33.085 & 6.770 & 15.0 & 137.5 & 90 & -0.6 & 1.2 & 0.0 & 0.0 & -0.3 & 1.4\end{array}$

$\begin{array}{llllllllllllllll}23 & 242.624 & 33.085 & 242.682 & 33.008 & 10.113 & 15.0 & 147.6 & 90 & -0.5 & 1.2 & 0.0 & 0.0 & -0.6 & 1.5\end{array}$

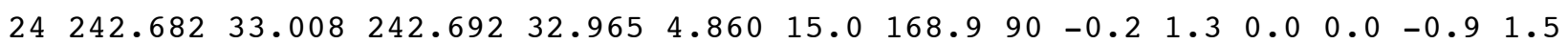

$\begin{array}{lllllllllllllll}25 & 242.692 & 32.965 & 242.739 & 32.858 & 12.655 & 15.0 & 159.7 & 90 & -0.4 & 1.2 & 0.0 & 0.0 & -1.1 & 1.6\end{array}$

$\begin{array}{lllllllllllllll}26 & 242.739 & 32.858 & 242.777 & 32.828 & 4.871 & 15.0 & 133.1 & 90 & -0.9 & 1.2 & 0.0 & 0.0 & -1.0 & 1.7\end{array}$

$\begin{array}{lllllllllllllll}27 & 242.777 & 32.828 & 242.812 & 32.764 & 7.818 & 15.0 & 155.2 & 90 & -0.4 & 1.2 & 0.0 & 0.0 & -1.4 & 1.8\end{array}$

$\begin{array}{lllllllllllllll}28 & 242.812 & 32.764 & 242.868 & 32.707 & 8.216 & 15.0 & 140.3 & 90 & -0.8 & 1.2 & 0.0 & 0.0 & -1.4 & 1.8\end{array}$

$\begin{array}{lllllllllllllll}29 & 242.868 & 32.707 & 242.918 & 32.580 & 14.845 & 15.0 & 161.6 & 90 & -0.2 & 1.3 & 0.0 & 0.0 & -1.9 & 1.9\end{array}$

$\begin{array}{lllllllllllllll}30 & 242.918 & 32.580 & 243.579 & 31.953 & 93.342 & 15.0 & 138.0 & 90 & -1.0 & 1.2 & 0.0 & 0.0 & -2.9 & 2.5\end{array}$

$\begin{array}{llllllllllllllll}31 & 243.579 & 31.953 & 244.239 & 31.325 & 93.640 & 15.0 & 137.9 & 90 & -1.1 & 1.2 & 0.0 & 0.0 & -5.1 & 3.4\end{array}$

Palos Verdes-Oceanside connector

$\begin{array}{lllllllllllllll}1 & 243.361 & 31.581 & 243.471 & 31.580 & 10.442 & 15.0 & 90.6 & 90 & 4.3 & 2.7 & 0.0 & 0.0 & -7.9 & 1.9\end{array}$

$\begin{array}{lllllllllllllll}2 & 243.471 & 31.580 & 243.546 & 31.571 & 7.189 & 15.0 & 98.0 & 90 & 5.2 & 2.5 & 0.0 & 0.0 & -7.2 & 2.2\end{array}$

$\begin{array}{llllllllllllllll}3 & 243.546 & 31.571 & 243.611 & 31.553 & 6.486 & 15.0 & 107.9 & 90 & 6.4 & 2.1 & 0.0 & 0.0 & -6.1 & 2.6\end{array}$

$\begin{array}{llllllllllllllll}4 & 243.611 & 31.553 & 243.677 & 31.527 & 6.899 & 15.0 & 114.7 & 90 & 7.1 & 1.9 & 0.0 & 0.0 & -5.2 & 2.8\end{array}$

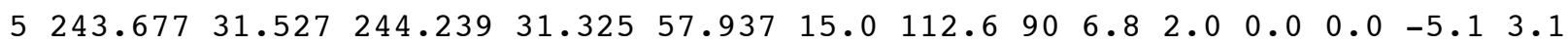

j. White Wolf

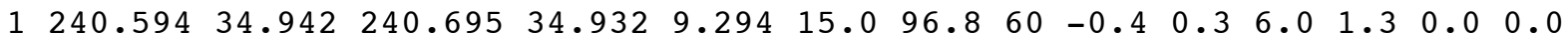

$\begin{array}{lllllllllllllllll}2 & 240.695 & 34.932 & 240.805 & 34.942 & 10.110 & 15.0 & 83.7 & 62 & -1.1 & 0.4 & 5.9 & 1.2 & 0.0 & 0.0\end{array}$

$\begin{array}{lllllllllllllll}3 & 240.805 & 34.942 & 240.884 & 34.985 & 8.649 & 15.0 & 56.5 & 48 & -2.2 & 0.5 & 2.9 & 0.6 & 0.0 & 0.0\end{array}$

$\begin{array}{lllllllllllllll}4 & 240.884 & 34.985 & 240.969 & 34.986 & 7.762 & 15.0 & 89.2 & 43 & -0.8 & 0.3 & 3.8 & 0.7 & 0.0 & 0.0\end{array}$

$\begin{array}{lllllllllllllll}5 & 240.969 & 34.986 & 240.993 & 35.029 & 5.249 & 15.0 & 24.7 & 75 & -2.8 & 0.6 & 1.6 & 1.0 & 0.0 & 0.0\end{array}$

$\begin{array}{lllllllllllllll}6 & 240.993 & 35.029 & 241.189 & 35.148 & 22.220 & 15.0 & 53.5 & 75 & -2.3 & 0.5 & 6.3 & 1.1 & 0.0 & 0.0\end{array}$

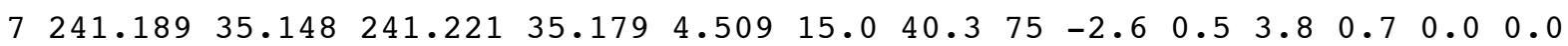

$\begin{array}{lllllllllllllll}8 & 241.221 & 35.179 & 241.265 & 35.211 & 5.354 & 15.0 & 48.4 & 75 & -2.4 & 0.5 & 5.0 & 0.8 & 0.0 & 0.0\end{array}$

$\begin{array}{llllllllllllllll}9 & 241.265 & 35.211 & 241.328 & 35.234 & 6.278 & 15.0 & 66.0 & 75 & -1.9 & 0.5 & 7.5 & 1.1 & 0.0 & 0.0\end{array}$

$\begin{array}{lllllllllllllll}10 & 241.328 & 35.234 & 241.398 & 35.269 & 7.461 & 15.0 & 58.6 & 75 & -2.2 & 0.5 & 6.3 & 0.9 & 0.0 & 0.0\end{array}$

$\begin{array}{lllllllllllllll}11 & 241.398 & 35.269 & 241.462 & 35.322 & 8.274 & 15.0 & 44.7 & 75 & -2.5 & 0.5 & 3.9 & 0.7 & 0.0 & 0.0\end{array}$

$\begin{array}{lllllllllllllll}12 & 241.462 & 35.322 & 241.513 & 35.373 & 7.315 & 15.0 & 39.3 & 75 & -2.6 & 0.5 & 2.8 & 0.8 & 0.0 & 0.0\end{array}$

$\begin{array}{lllllllllllllll}13 & 241.513 & 35.373 & 241.893 & 35.674 & 47.992 & 15.0 & 45.8 & 75 & -2.5 & 0.5 & 3.2 & 1.1 & 0.0 & 0.0\end{array}$

$\begin{array}{lllllllllllllll}14 & 241.893 & 35.674 & 241.995 & 35.770 & 14.094 & 15.0 & 40.9 & 75 & -2.5 & 0.5 & 1.5 & 1.8 & 0.0 & 0.0\end{array}$

$\begin{array}{lllllllllllllll}15 & 241.995 & 35.770 & 242.105 & 35.880 & 15.741 & 15.0 & 39.1 & 75 & -2.5 & 0.5 & 0.9 & 2.1 & 0.0 & 0.0\end{array}$ 
\# Lon-1 Lat-1 Lon-2 Lat-2 L(km) $\mathrm{Z}(\mathrm{km})$ strike dip U_S S_S U_d S_d U_t S_t

k. Garlock (west)

$\begin{array}{llllllllllllllll}1 & 241.103 & 34.811 & 241.169 & 34.845 & 7.119 & 15.0 & 58.0 & 90 & -1.7 & 0.5 & 0.0 & 0.0 & -6.2 & 0.5\end{array}$

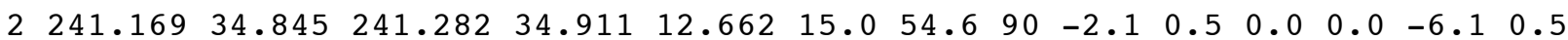

$\begin{array}{lllllllllllllll}3 & 241.282 & 34.911 & 241.525 & 34.996 & 24.116 & 15.0 & 66.9 & 90 & -0.7 & 0.5 & 0.0 & 0.0 & -6.3 & 0.5\end{array}$

$\begin{array}{lllllllllllllll}4 & 241.525 & 34.996 & 241.652 & 35.081 & 14.940 & 15.0 & 50.8 & 90 & -2.5 & 0.5 & 0.0 & 0.0 & -5.8 & 0.3\end{array}$

$\begin{array}{llllllllllllllll}5 & 241.652 & 35.081 & 241.910 & 35.209 & 27.467 & 15.0 & 58.8 & 90 & -1.6 & 0.5 & 0.0 & 0.0 & -6.0 & 0.3\end{array}$

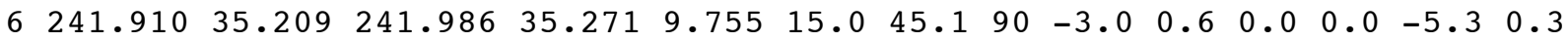

1. Garlock (central)

$\begin{array}{lllllllllllllll}1 & 241.977 & 35.284 & 242.187 & 35.398 & 22.900 & 15.0 & 56.4 & 90 & -3.8 & 2.1 & 0.0 & 0.0 & 3.5 & 2.5\end{array}$

$\begin{array}{llllllllllllllll}2 & 242.187 & 35.398 & 242.309 & 35.441 & 12.063 & 15.0 & 66.7 & 90 & -4.1 & 2.2 & 0.0 & 0.0 & 0.7 & 1.6\end{array}$

$\begin{array}{lllllllllllllll}3 & 242.309 & 35.441 & 242.448 & 35.486 & 13.569 & 15.0 & 68.4 & 90 & -4.1 & 2.2 & 0.0 & 0.0 & -0.9 & 2.0\end{array}$

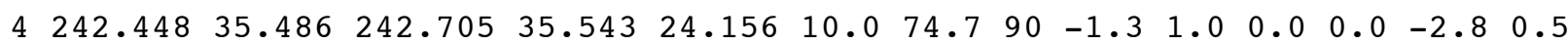

$\begin{array}{lllllllllllllll}5 & 242.705 & 35.543 & 242.861 & 35.585 & 14.890 & 10.0 & 71.7 & 90 & -1.4 & 1.0 & 0.0 & 0.0 & -2.7 & 0.4\end{array}$

$\begin{array}{lllllllllllllll}6 & 242.861 & 35.585 & 242.992 & 35.609 & 12.166 & 10.0 & 77.3 & 90 & -1.2 & 0.9 & 0.0 & 0.0 & -2.7 & 0.5\end{array}$

Garlock (east)

$\begin{array}{lllllllllllllllll}1 & 242.992 & 35.609 & 243.111 & 35.603 & 10.803 & 10.0 & 93.5 & 90 & -4.0 & 0.8 & 0.0 & 0.0 & 2.5 & 0.8\end{array}$

$\begin{array}{lllllllllllllll}2 & 243.111 & 35.603 & 243.220 & 35.609 & 9.899 & 10.0 & 86.1 & 90 & -1.1 & 0.5 & 0.0 & 0.0 & 0.6 & 0.5\end{array}$

$\begin{array}{llllllllllllllll}3 & 243.220 & 35.609 & 243.301 & 35.600 & 7.407 & 10.0 & 97.7 & 90 & -1.2 & 0.5 & 0.0 & 0.0 & 0.4 & 0.5\end{array}$

$\begin{array}{lllllllllllllll}4 & 243.301 & 35.600 & 243.608 & 35.596 & 27.824 & 10.0 & 90.8 & 90 & -1.1 & 0.5 & 0.0 & 0.0 & 0.4 & 0.6\end{array}$

Garlock (east extension)

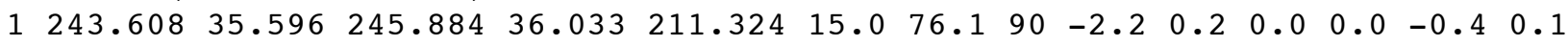

North Frontal

$\begin{array}{lllllllllllllllll}1 & 242.470 & 34.311 & 242.537 & 34.312 & 6.168 & 15.0 & 89.0 & 90 & -2.0 & 0.1 & 0.0 & 0.0 & 6.9 & 0.4\end{array}$

$\begin{array}{llllllllllllllll}2 & 242.537 & 34.312 & 242.612 & 34.286 & 7.483 & 15.0 & 112.6 & 90 & -4.6 & 0.2 & 0.0 & 0.0 & 5.0 & 0.3\end{array}$

$\begin{array}{llllllllllllllll}3 & 242.612 & 34.286 & 242.649 & 34.288 & 3.414 & 15.0 & 86.3 & 90 & -2.0 & 0.1 & 0.0 & 0.0 & 6.2 & 0.3\end{array}$

$\begin{array}{lllllllllllllll}4 & 242.649 & 34.288 & 242.665 & 34.306 & 2.481 & 15.0 & 36.4 & 90 & 3.4 & 0.2 & 0.0 & 0.0 & 5.3 & 0.3\end{array}$

$\begin{array}{llllllllllllllllll}5 & 242.665 & 34.306 & 242.696 & 34.316 & 3.061 & 15.0 & 68.7 & 90 & 0.1 & 0.0 & 0.0 & 0.0 & 6.1 & 0.3\end{array}$

$\begin{array}{llllllllllllllllll}6 & 242.696 & 34.316 & 242.731 & 34.318 & 3.229 & 15.0 & 86.1 & 90 & -1.7 & 0.1 & 0.0 & 0.0 & 5.6 & 0.3\end{array}$

$\begin{array}{lllllllllllllllll}7 & 242.731 & 34.318 & 242.799 & 34.371 & 8.586 & 15.0 & 46.8 & 56 & 2.1 & 0.1 & -8.9 & 0.5 & 0.0 & 0.0\end{array}$

$\begin{array}{lllllllllllllllll}8 & 242.799 & 34.371 & 242.855 & 34.444 & 9.596 & 15.0 & 32.4 & 51 & 3.2 & 0.2 & -5.8 & 0.3 & 0.0 & 0.0\end{array}$

$\begin{array}{llllllllllllllll}9 & 242.855 & 34.444 & 242.940 & 34.422 & 8.185 & 15.0 & 107.3 & 48 & -2.3 & 0.1 & -5.5 & 0.3 & 0.0 & 0.0\end{array}$

$\begin{array}{lllllllllllllll}10 & 242.940 & 34.422 & 243.011 & 34.382 & 7.893 & 15.0 & 124.2 & 54 & -3.2 & 0.2 & -3.8 & 0.2 & 0.0 & 0.0\end{array}$

$\begin{array}{llllllllllllllll}11 & 243.011 & 34.382 & 243.062 & 34.365 & 5.056 & 15.0 & 111.9 & 50 & -2.7 & 0.1 & -3.7 & 0.2 & 0.0 & 0.0\end{array}$

$\begin{array}{llllllllllllllll}12 & 243.062 & 34.365 & 243.126 & 34.367 & 5.891 & 15.0 & 87.8 & 44 & -1.6 & 0.1 & -4.0 & 0.2 & 0.0 & 0.0\end{array}$

$\begin{array}{lllllllllllllll}13 & 243.126 & 34.367 & 243.143 & 34.377 & 1.917 & 15.0 & 54.6 & 90 & 0.2 & 0.0 & 0.0 & 0.0 & 3.0 & 0.2\end{array}$

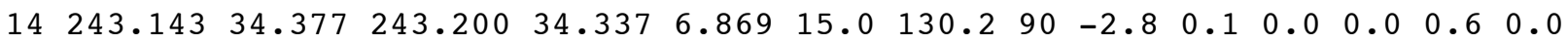

$\begin{array}{lllllllllllllllll}15 & 243.200 & 34.337 & 243.290 & 34.346 & 8.341 & 15.0 & 83.1 & 42 & -1.7 & 0.1 & -2.7 & 0.1 & 0.0 & 0.0\end{array}$

$\begin{array}{lllllllllllllllll}16 & 243.290 & 34.346 & 243.357 & 34.340 & 6.201 & 15.0 & 96.1 & 39 & -2.0 & 0.1 & -1.3 & 0.1 & 0.0 & 0.0\end{array}$

$\begin{array}{lllllllllllllllll}17 & 243.357 & 34.340 & 243.411 & 34.321 & 5.398 & 15.0 & 113.0 & 41 & -2.2 & 0.1 & -0.0 & 0.0 & 0.0 & 0.0\end{array}$

$\begin{array}{llllllllllllllll}18 & 243.411 & 34.321 & 243.475 & 34.310 & 6.016 & 15.0 & 101.7 & 42 & -2.2 & 0.1 & 0.0 & 0.0 & 0.0 & 0.0\end{array}$

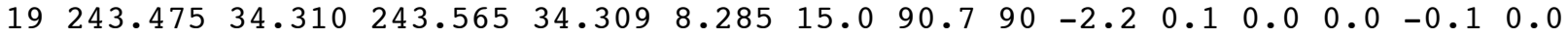

\section{Eureka Peak}

$\begin{array}{lllllllllllllll}1 & 243.580 & 34.131 & 243.638 & 34.066 & 8.979 & 15.0 & 143.4 & 90 & 15.1 & 0.2 & 0.0 & 0.0 & -2.5 & 0.1\end{array}$

$\begin{array}{lllllllllllllllll}2 & 243.638 & 34.066 & 243.671 & 34.008 & 7.119 & 15.0 & 154.6 & 90 & 15.4 & 0.2 & 0.0 & 0.0 & 0.0 & 0.0\end{array}$

$\begin{array}{llllllllllllllll}3 & 243.671 & 34.008 & 243.817 & 33.794 & 27.309 & 15.0 & 150.3 & 90 & 15.3 & 0.2 & 0.0 & 0.0 & -2.3 & 0.0\end{array}$

$\begin{array}{lllllllllllllll}4 & 243.817 & 33.794 & 243.876 & 33.699 & 11.871 & 15.0 & 152.6 & 90 & 15.4 & 0.2 & 0.0 & 0.0 & -3.0 & 0.1\end{array}$

Pinto Mountain

$\begin{array}{lllllllllllllll}1 & 243.580 & 34.131 & 243.712 & 34.143 & 12.248 & 15.0 & 83.7 & 90 & -7.2 & 0.3 & 0.0 & 0.0 & 8.1 & 0.3\end{array}$

$\begin{array}{lllllllllllllll}2 & 243.712 & 34.143 & 243.765 & 34.145 & 4.893 & 15.0 & 87.4 & 90 & -7.7 & 0.3 & 0.0 & 0.0 & 7.5 & 0.3\end{array}$

$\begin{array}{llllllllllllllll}3 & 243.765 & 34.145 & 243.849 & 34.142 & 7.754 & 15.0 & 92.4 & 90 & -8.3 & 0.3 & 0.0 & 0.0 & 6.7 & 0.4\end{array}$

$\begin{array}{lllllllllllllll}4 & 243.849 & 34.142 & 244.021 & 34.116 & 16.126 & 15.0 & 100.3 & 90 & -8.7 & 0.4 & 0.0 & 0.0 & 5.0 & 0.3\end{array}$ 
\# Lon-1 Lat-1 Lon-2 Lat-2 L(km) $\mathrm{Z}(\mathrm{km})$ strike dip U_S S_S U_d S_d U_t S_t

o. Johnson Valley-Lockhart

$\begin{array}{llllllllllllllllll}1 & 241.986 & 35.271 & 242.403 & 35.110 & 41.969 & 10.0 & 115.1 & 90 & 5.3 & 0.4 & 0.0 & 0.0 & -2.9 & 0.6\end{array}$

$\begin{array}{lllllllllllllll}2 & 242.403 & 35.110 & 242.488 & 35.076 & 8.620 & 10.0 & 115.9 & 90 & 5.3 & 0.4 & 0.0 & 0.0 & -2.9 & 0.5\end{array}$

$\begin{array}{lllllllllllllllll}3 & 242.488 & 35.076 & 242.628 & 35.008 & 14.835 & 10.0 & 120.5 & 90 & 5.5 & 0.4 & 0.0 & 0.0 & -2.6 & 0.5\end{array}$

$\begin{array}{llllllllllllllll}4 & 242.628 & 35.008 & 242.793 & 34.929 & 17.432 & 10.0 & 120.1 & 90 & 5.5 & 0.4 & 0.0 & 0.0 & -2.7 & 0.4\end{array}$

$\begin{array}{llllllllllllllll}5 & 242.793 & 34.929 & 242.919 & 34.868 & 13.358 & 10.0 & 120.4 & 90 & 5.5 & 0.4 & 0.0 & 0.0 & -2.8 & 0.4\end{array}$

$\begin{array}{lllllllllllllll}6 & 242.919 & 34.868 & 243.072 & 34.765 & 18.070 & 10.0 & 129.2 & 90 & 5.9 & 0.4 & 0.0 & 0.0 & -2.0 & 0.3\end{array}$

$\begin{array}{lllllllllllllll}7 & 243.072 & 34.765 & 243.317 & 34.635 & 26.680 & 10.0 & 122.6 & 90 & 5.6 & 0.4 & 0.0 & 0.0 & -2.8 & 0.2\end{array}$

$\begin{array}{llllllllllllllll}8 & 243.317 & 34.635 & 243.423 & 34.565 & 12.444 & 10.0 & 128.6 & 90 & 5.8 & 0.4 & 0.0 & 0.0 & -2.3 & 0.2\end{array}$

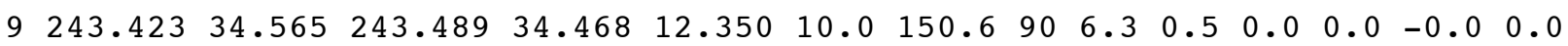

$\begin{array}{lllllllllllllll}10 & 243.489 & 34.468 & 243.565 & 34.309 & 18.972 & 10.0 & 158.4 & 90 & 6.2 & 0.5 & 0.0 & 0.0 & 0.8 & 0.1\end{array}$

$\begin{array}{lllllllllllllll}11 & 243.565 & 34.309 & 243.571 & 34.275 & 3.812 & 15.0 & 171.7 & 90 & 6.0 & 0.5 & 0.0 & 0.0 & -0.2 & 0.3\end{array}$

$\begin{array}{lllllllllllllllll}12 & 243.571 & 34.195 & 243.571 & 34.275 & 8.874 & 15.0 & 0.0 & 90 & 6.0 & 0.4 & 0.0 & 0.0 & 0.1 & 0.3\end{array}$

$\begin{array}{lllllllllllllll}13 & 243.571 & 34.195 & 243.579 & 34.170 & 2.870 & 15.0 & 165.1 & 90 & 5.7 & 0.5 & 0.0 & 0.0 & -1.9 & 0.3\end{array}$

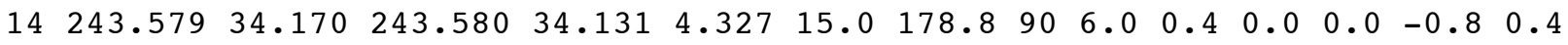

\section{p. Calico-Blackwater}

$\begin{array}{llllllllllllllll}1 & 242.448 & 35.486 & 242.587 & 35.451 & 13.200 & 5.0 & 107.1 & 90 & 0.4 & 0.4 & 0.0 & 0.0 & -0.4 & 0.4\end{array}$

$\begin{array}{lllllllllllllllll}2 & 242.587 & 35.451 & 242.687 & 35.368 & 12.935 & 5.0 & 135.4 & 90 & 0.5 & 0.5 & 0.0 & 0.0 & -0.2 & 0.1\end{array}$

$\begin{array}{llllllllllllllllll}3 & 242.687 & 35.368 & 242.786 & 35.229 & 17.858 & 5.0 & 149.7 & 90 & 0.6 & 0.5 & 0.0 & 0.0 & 0.0 & 0.0\end{array}$

$\begin{array}{lllllllllllllllll}4 & 242.786 & 35.229 & 242.872 & 35.108 & 15.543 & 5.0 & 149.7 & 90 & 0.6 & 0.5 & 0.0 & 0.0 & 0.0 & 0.0\end{array}$

$\begin{array}{llllllllllllllll}5 & 242.872 & 35.108 & 242.967 & 35.049 & 10.859 & 5.0 & 127.0 & 90 & 0.5 & 0.5 & 0.0 & 0.0 & -0.2 & 0.2\end{array}$

$\begin{array}{lllllllllllllllll}6 & 242.967 & 35.049 & 243.063 & 34.990 & 10.937 & 5.0 & 126.7 & 90 & 0.5 & 0.5 & 0.0 & 0.0 & -0.2 & 0.2\end{array}$

$\begin{array}{llllllllllllllll}7 & 243.063 & 34.990 & 243.115 & 34.954 & 6.205 & 5.0 & 130.1 & 90 & 0.5 & 0.5 & 0.0 & 0.0 & -0.2 & 0.1\end{array}$

$\begin{array}{lllllllllllllllll}8 & 243.115 & 34.954 & 243.233 & 34.922 & 11.350 & 5.0 & 108.2 & 90 & 0.4 & 0.4 & 0.0 & 0.0 & -0.3 & 0.3\end{array}$

$\begin{array}{llllllllllllllll}9 & 243.233 & 34.922 & 243.333 & 34.836 & 13.214 & 5.0 & 136.2 & 90 & 0.5 & 0.5 & 0.0 & 0.0 & -0.1 & 0.1\end{array}$

$\begin{array}{llllllllllllllll}10 & 243.333 & 34.836 & 243.381 & 34.768 & 8.729 & 5.0 & 149.8 & 90 & 0.5 & 0.5 & 0.0 & 0.0 & 0.1 & 0.1\end{array}$

$\begin{array}{llllllllllllllll}11 & 243.381 & 34.768 & 243.472 & 34.668 & 13.876 & 5.0 & 143.1 & 90 & 0.5 & 0.5 & 0.0 & 0.0 & 0.0 & 0.0\end{array}$

$\begin{array}{lllllllllllllllll}12 & 243.472 & 34.668 & 243.551 & 34.586 & 11.629 & 5.0 & 141.4 & 90 & 0.5 & 0.5 & 0.0 & 0.0 & -0.0 & 0.0\end{array}$

$\begin{array}{llllllllllllllll}13 & 243.551 & 34.586 & 243.664 & 34.478 & 15.848 & 5.0 & 139.1 & 90 & 0.5 & 0.5 & 0.0 & 0.0 & -0.0 & 0.0\end{array}$

$\begin{array}{llllllllllllllll}14 & 243.664 & 34.478 & 243.672 & 34.435 & 4.826 & 5.0 & 171.2 & 90 & 0.5 & 0.4 & 0.0 & 0.0 & 0.3 & 0.3\end{array}$

$\begin{array}{lllllllllllllllll}15 & 243.672 & 34.435 & 243.729 & 34.389 & 7.314 & 5.0 & 134.2 & 90 & 0.5 & 0.5 & 0.0 & 0.0 & -0.0 & 0.0\end{array}$

$\begin{array}{llllllllllllllll}16 & 243.729 & 34.389 & 243.751 & 34.335 & 6.323 & 5.0 & 161.3 & 90 & 0.5 & 0.5 & 0.0 & 0.0 & 0.2 & 0.2\end{array}$

$\begin{array}{llllllllllllllll}17 & 243.751 & 34.335 & 243.777 & 34.296 & 4.944 & 5.0 & 151.0 & 90 & 0.5 & 0.5 & 0.0 & 0.0 & 0.1 & 0.1\end{array}$

$\begin{array}{lllllllllllllllll}18 & 243.777 & 34.296 & 243.803 & 34.231 & 7.597 & 5.0 & 161.6 & 90 & 0.5 & 0.5 & 0.0 & 0.0 & 0.2 & 0.2\end{array}$

$\begin{array}{llllllllllllllll}19 & 243.803 & 34.231 & 243.849 & 34.142 & 10.745 & 5.0 & 156.7 & 90 & 0.5 & 0.5 & 0.0 & 0.0 & 0.2 & 0.2\end{array}$

q. Goldstone-Billion

$\begin{array}{llllllllllllllll}1 & 242.992 & 35.609 & 243.032 & 35.489 & 13.799 & 10.0 & 164.7 & 90 & 6.3 & 0.5 & 0.0 & 0.0 & 1.8 & 0.2\end{array}$

$\begin{array}{llllllllllllllll}2 & 243.032 & 35.489 & 243.075 & 35.455 & 5.428 & 10.0 & 134.0 & 90 & 6.4 & 0.5 & 0.0 & 0.0 & -1.6 & 0.1\end{array}$

$\begin{array}{lllllllllllllllll}3 & 243.075 & 35.455 & 243.118 & 35.397 & 7.527 & 10.0 & 148.7 & 90 & 6.6 & 0.6 & 0.0 & 0.0 & 0.2 & 0.0\end{array}$

$\begin{array}{llllllllllllllll}4 & 243.118 & 35.397 & 243.262 & 35.226 & 23.053 & 10.0 & 145.3 & 90 & 6.6 & 0.6 & 0.0 & 0.0 & -0.0 & 0.0\end{array}$

$\begin{array}{lllllllllllllllll}5 & 243.262 & 35.226 & 243.309 & 35.179 & 6.746 & 10.0 & 140.6 & 90 & 6.6 & 0.6 & 0.0 & 0.0 & -0.4 & 0.0\end{array}$

$\begin{array}{llllllllllllllll}6 & 243.309 & 35.179 & 243.517 & 34.814 & 44.724 & 10.0 & 154.8 & 90 & 6.5 & 0.5 & 0.0 & 0.0 & 1.6 & 0.1\end{array}$

$\begin{array}{lllllllllllllllll}7 & 243.517 & 34.814 & 243.553 & 34.767 & 6.168 & 10.0 & 147.7 & 90 & 6.7 & 0.6 & 0.0 & 0.0 & 1.1 & 0.1\end{array}$

$\begin{array}{lllllllllllllllll}8 & 243.553 & 34.767 & 243.602 & 34.685 & 10.144 & 10.0 & 153.7 & 90 & 6.5 & 0.5 & 0.0 & 0.0 & 1.9 & 0.2\end{array}$

$\begin{array}{llllllllllllllll}9 & 243.602 & 34.685 & 243.616 & 34.636 & 5.585 & 10.0 & 166.7 & 90 & 5.9 & 0.5 & 0.0 & 0.0 & 3.4 & 0.3\end{array}$

$\begin{array}{lllllllllllllll}10 & 243.616 & 34.636 & 243.626 & 34.587 & 5.513 & 10.0 & 170.4 & 90 & 5.7 & 0.5 & 0.0 & 0.0 & 3.8 & 0.3\end{array}$

$\begin{array}{llllllllllllllll}11 & 243.626 & 34.587 & 243.742 & 34.489 & 15.218 & 10.0 & 135.6 & 90 & 6.9 & 0.6 & 0.0 & 0.0 & -0.0 & 0.0\end{array}$

$\begin{array}{llllllllllllllll}12 & 243.742 & 34.489 & 243.873 & 34.359 & 18.788 & 10.0 & 140.1 & 90 & 6.8 & 0.6 & 0.0 & 0.0 & 0.7 & 0.1\end{array}$

$\begin{array}{lllllllllllllll}13 & 243.873 & 34.359 & 243.910 & 34.247 & 12.882 & 10.0 & 164.7 & 90 & 5.9 & 0.5 & 0.0 & 0.0 & 3.7 & 0.3\end{array}$

$\begin{array}{llllllllllllllll}14 & 243.910 & 34.247 & 243.987 & 34.143 & 13.545 & 10.0 & 148.4 & 90 & 6.7 & 0.6 & 0.0 & 0.0 & 2.1 & 0.2\end{array}$

$\begin{array}{lllllllllllllll}15 & 243.987 & 34.143 & 244.021 & 34.116 & 4.337 & 15.0 & 133.7 & 90 & 7.0 & 0.6 & 0.0 & 0.0 & 0.4 & 0.0\end{array}$

r. Ludlow

$\begin{array}{llllllllllllllllll}1 & 243.608 & 35.596 & 243.643 & 35.246 & 38.962 & 10.0 & 175.3 & 90 & 2.1 & 0.3 & 0.0 & 0.0 & 1.7 & 0.3\end{array}$

$\begin{array}{llllllllllllllll}2 & 243.643 & 35.246 & 243.699 & 35.173 & 9.570 & 10.0 & 147.8 & 90 & 2.7 & 0.3 & 0.0 & 0.0 & 0.6 & 0.2\end{array}$

$\begin{array}{lllllllllllllll}3 & 243.699 & 35.173 & 243.818 & 35.067 & 15.999 & 10.0 & 137.3 & 90 & 2.7 & 0.3 & 0.0 & 0.0 & 0.2 & 0.1\end{array}$ 
\# Lon-1 Lat-1 Lon-2 Lat-2 L(km) $\mathrm{Z}(\mathrm{km})$ strike dip U_S S_S U_d S_d U_t S_t

$\begin{array}{llllllllllllllll}4 & 243.749 & 34.961 & 243.818 & 35.067 & 13.340 & 10.0 & 28.2 & 90 & 0.7 & 0.1 & 0.0 & 0.0 & 2.7 & 0.3\end{array}$ $\begin{array}{lllllllllllllllll}5 & 243.749 & 34.961 & 243.797 & 34.909 & 7.246 & 10.0 & 142.7 & 90 & 2.7 & 0.3 & 0.0 & 0.0 & 0.5 & 0.1\end{array}$ $\begin{array}{llllllllllllllll}6 & 243.783 & 34.826 & 243.797 & 34.909 & 9.296 & 10.0 & 7.9 & 90 & 1.6 & 0.2 & 0.0 & 0.0 & 2.3 & 0.3\end{array}$ $\begin{array}{llllllllllllllll}7 & 243.783 & 34.826 & 243.811 & 34.785 & 5.220 & 10.0 & 150.6 & 90 & 2.7 & 0.3 & 0.0 & 0.0 & 0.9 & 0.2\end{array}$ $\begin{array}{llllllllllllllll}8 & 243.811 & 34.785 & 243.839 & 34.707 & 9.025 & 10.0 & 163.5 & 90 & 2.4 & 0.3 & 0.0 & 0.0 & 1.5 & 0.2\end{array}$ $\begin{array}{lllllllllllllll}9 & 243.839 & 34.707 & 244.123 & 34.443 & 39.202 & 10.0 & 138.3 & 90 & 2.8 & 0.3 & 0.0 & 0.0 & 0.4 & 0.2\end{array}$

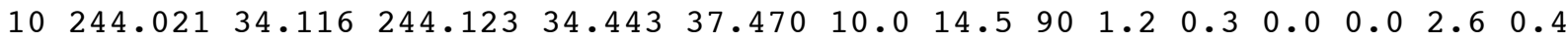

\section{Sierra Nevada}

$\begin{array}{llllllllllllllll}1 & 242.046 & 35.973 & 242.105 & 35.880 & 11.612 & 10.0 & 152.7 & 90 & -0.5 & 1.7 & 0.0 & 0.0 & 7.9 & 4.1\end{array}$

$\begin{array}{llllllllllllllll}2 & 242.096 & 35.761 & 242.105 & 35.880 & 13.229 & 10.0 & 3.5 & 90 & -1.9 & 1.6 & 0.0 & 0.0 & 3.7 & 3.4\end{array}$

$\begin{array}{lllllllllllllll}3 & 242.096 & 35.761 & 242.131 & 35.686 & 8.904 & 10.0 & 159.2 & 90 & -0.6 & 1.6 & 0.0 & 0.0 & 2.8 & 2.5\end{array}$

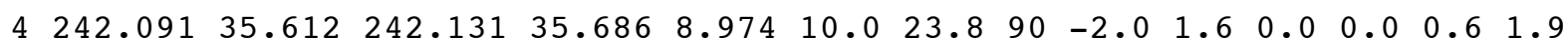

$\begin{array}{llllllllllllllll}5 & 241.996 & 35.570 & 242.091 & 35.612 & 9.790 & 10.0 & 61.5 & 90 & -1.6 & 1.8 & 0.0 & 0.0 & -1.8 & 1.8\end{array}$

$\begin{array}{lllllllllllllll}6 & 241.966 & 35.540 & 241.996 & 35.570 & 4.299 & 10.0 & 39.2 & 90 & -2.4 & 1.9 & 0.0 & 0.0 & -1.9 & 1.9\end{array}$

$\begin{array}{lllllllllllllll}7 & 241.949 & 35.428 & 241.966 & 35.540 & 12.522 & 10.0 & 7.1 & 90 & -3.2 & 2.2 & 0.0 & 0.0 & -1.4 & 1.9\end{array}$

$\begin{array}{lllllllllllllll}8 & 241.949 & 35.428 & 241.960 & 35.349 & 8.822 & 10.0 & 173.5 & 90 & -3.6 & 2.5 & 0.0 & 0.0 & -2.0 & 2.2\end{array}$ $\begin{array}{llllllllllllllll}9 & 241.960 & 35.349 & 241.971 & 35.298 & 5.746 & 10.0 & 170.0 & 90 & -3.8 & 2.6 & 0.0 & 0.0 & -2.7 & 2.6\end{array}$ $\begin{array}{lllllllllllllll}10 & 241.971 & 35.298 & 241.977 & 35.284 & 1.646 & 10.0 & 160.6 & 90 & -4.2 & 2.9 & 0.0 & 0.0 & -2.5 & 2.5\end{array}$ $\begin{array}{lllllllllllllll}11 & 241.977 & 35.284 & 241.986 & 35.271 & 1.659 & 10.0 & 150.4 & 90 & -0.1 & 0.5 & 0.0 & 0.0 & 2.3 & 0.6\end{array}$

\section{Owens Valley}

$\begin{array}{lllllllllllllllll}1 & 241.573 & 37.793 & 241.620 & 37.951 & 18.018 & 10.0 & 13.3 & 90 & 2.4 & 1.4 & 0.0 & 0.0 & 1.2 & 1.8\end{array}$

$\begin{array}{llllllllllllllll}2 & 241.573 & 37.793 & 241.596 & 37.434 & 39.897 & 10.0 & 177.1 & 90 & 2.6 & 1.0 & 0.0 & 0.0 & 0.0 & 2.0\end{array}$

$\begin{array}{lllllllllllllllll}3 & 241.596 & 37.434 & 241.671 & 37.236 & 22.958 & 10.0 & 163.1 & 90 & 2.5 & 0.7 & 0.0 & 0.0 & -1.1 & 2.2\end{array}$

$\begin{array}{llllllllllllllll}4 & 241.671 & 37.236 & 241.803 & 36.933 & 35.616 & 10.0 & 160.7 & 90 & 2.4 & 0.7 & 0.0 & 0.0 & -1.7 & 2.3\end{array}$

$\begin{array}{lllllllllllllll}5 & 241.803 & 36.933 & 241.840 & 36.852 & 9.575 & 10.0 & 159.8 & 90 & 2.4 & 0.7 & 0.0 & 0.0 & -2.2 & 2.5\end{array}$

$\begin{array}{llllllllllllllll}6 & 241.840 & 36.852 & 241.881 & 36.776 & 9.193 & 10.0 & 156.5 & 90 & 2.2 & 0.6 & 0.0 & 0.0 & -2.5 & 2.6\end{array}$

$\begin{array}{lllllllllllllll}7 & 241.881 & 36.776 & 241.917 & 36.696 & 9.442 & 10.0 & 160.1 & 90 & 2.4 & 0.7 & 0.0 & 0.0 & -2.5 & 2.7\end{array}$

$\begin{array}{lllllllllllllllll}8 & 241.917 & 36.696 & 241.930 & 36.637 & 6.650 & 10.0 & 169.9 & 90 & 2.8 & 0.9 & 0.0 & 0.0 & -2.2 & 2.7\end{array}$

$\begin{array}{lllllllllllllllll}9 & 241.930 & 36.637 & 241.957 & 36.554 & 9.522 & 10.0 & 165.3 & 90 & 2.6 & 0.8 & 0.0 & 0.0 & -2.5 & 2.8\end{array}$

$\begin{array}{llllllllllllllll}10 & 241.957 & 36.554 & 241.980 & 36.507 & 5.608 & 10.0 & 158.4 & 90 & 2.3 & 0.6 & 0.0 & 0.0 & -3.0 & 2.9\end{array}$

$\begin{array}{lllllllllllllll}11 & 241.967 & 36.410 & 241.980 & 36.507 & 10.827 & 10.0 & 6.2 & 90 & 3.4 & 1.5 & 0.0 & 0.0 & -1.7 & 2.6\end{array}$

$\begin{array}{llllllllllllllll}12 & 241.967 & 36.410 & 241.977 & 36.350 & 6.718 & 10.0 & 172.3 & 90 & 1.0 & 0.4 & 0.0 & 0.0 & 4.2 & 0.4\end{array}$

$\begin{array}{lllllllllllllll}13 & 241.960 & 36.295 & 241.977 & 36.350 & 6.291 & 10.0 & 14.0 & 90 & -0.6 & 0.4 & 0.0 & 0.0 & 4.2 & 0.3\end{array}$

$\begin{array}{lllllllllllllll}14 & 241.960 & 36.295 & 242.010 & 36.221 & 9.360 & 10.0 & 151.3 & 90 & 2.4 & 0.4 & 0.0 & 0.0 & 3.4 & 0.4\end{array}$

$\begin{array}{lllllllllllllll}15 & 241.991 & 36.126 & 242.010 & 36.221 & 10.679 & 10.0 & 9.2 & 90 & -0.2 & 0.4 & 0.0 & 0.0 & 4.1 & 0.4\end{array}$

$\begin{array}{llllllllllllllll}16 & 241.991 & 36.126 & 242.015 & 36.082 & 5.339 & 10.0 & 156.1 & 90 & 2.0 & 0.4 & 0.0 & 0.0 & 3.4 & 0.4\end{array}$

$\begin{array}{lllllllllllllll}17 & 242.015 & 36.082 & 242.046 & 35.973 & 12.413 & 10.0 & 167.0 & 90 & 1.3 & 0.4 & 0.0 & 0.0 & 3.6 & 0.4\end{array}$

$\begin{array}{lllllllllllllll}18 & 242.046 & 35.973 & 242.119 & 35.897 & 10.701 & 10.0 & 142.0 & 90 & 1.6 & 2.4 & 0.0 & 0.0 & -5.3 & 3.9\end{array}$

$\begin{array}{lllllllllllllll}19 & 242.119 & 35.897 & 242.171 & 35.834 & 8.421 & 10.0 & 146.1 & 90 & 1.9 & 2.2 & 0.0 & 0.0 & -4.0 & 3.2\end{array}$

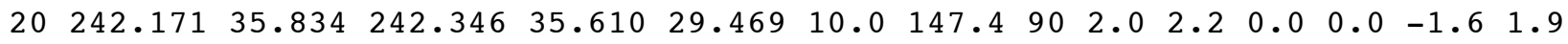

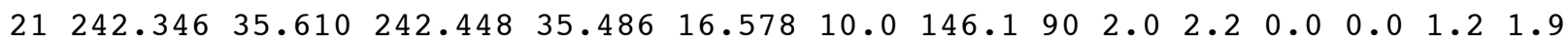

\section{m. Panamint Valley}

$\begin{array}{llllllllllllllll}1 & 241.946 & 37.150 & 242.060 & 37.483 & 38.314 & 10.0 & 15.3 & 90 & 1.7 & 1.2 & 0.0 & 0.0 & 2.8 & 1.4\end{array}$

$\begin{array}{llllllllllllllll}2 & 241.946 & 37.150 & 242.048 & 36.925 & 26.568 & 10.0 & 160.0 & 90 & 3.0 & 0.6 & 0.0 & 0.0 & 1.4 & 1.5\end{array}$

$\begin{array}{llllllllllllllll}3 & 242.048 & 36.925 & 242.106 & 36.773 & 17.644 & 10.0 & 162.9 & 90 & 2.9 & 0.7 & 0.0 & 0.0 & 1.6 & 1.2\end{array}$

$\begin{array}{llllllllllllllll}4 & 242.106 & 36.773 & 242.139 & 36.739 & 4.787 & 10.0 & 142.0 & 90 & 3.3 & 0.5 & 0.0 & 0.0 & 0.5 & 1.2\end{array}$

$\begin{array}{llllllllllllllllll}5 & 242.139 & 36.739 & 242.180 & 36.674 & 8.090 & 10.0 & 153.1 & 90 & 3.1 & 0.6 & 0.0 & 0.0 & 1.1 & 1.1\end{array}$

$\begin{array}{llllllllllllllllll}6 & 242.180 & 36.674 & 242.294 & 36.636 & 11.031 & 10.0 & 112.4 & 90 & 3.1 & 0.7 & 0.0 & 0.0 & -1.2 & 1.0\end{array}$

$\begin{array}{llllllllllllllll}7 & 242.294 & 36.636 & 242.342 & 36.615 & 4.885 & 10.0 & 118.5 & 90 & 3.2 & 0.7 & 0.0 & 0.0 & -0.8 & 1.0\end{array}$

$\begin{array}{lllllllllllllllll}8 & 242.342 & 36.615 & 242.388 & 36.574 & 6.135 & 10.0 & 137.9 & 90 & 3.3 & 0.6 & 0.0 & 0.0 & 0.3 & 1.0\end{array}$

$\begin{array}{lllllllllllllllll}9 & 242.388 & 36.574 & 242.437 & 36.540 & 5.786 & 10.0 & 130.7 & 90 & 3.3 & 0.6 & 0.0 & 0.0 & -0.1 & 0.9\end{array}$

$\begin{array}{llllllllllllllll}10 & 242.437 & 36.540 & 242.525 & 36.498 & 9.157 & 10.0 & 120.6 & 90 & 3.3 & 0.6 & 0.0 & 0.0 & -0.6 & 0.9\end{array}$

$\begin{array}{llllllllllllllll}11 & 242.525 & 36.498 & 242.592 & 36.436 & 9.132 & 10.0 & 138.9 & 90 & 3.3 & 0.6 & 0.0 & 0.0 & 0.5 & 0.9\end{array}$

$\begin{array}{llllllllllllllll}12 & 242.592 & 36.436 & 242.640 & 36.366 & 8.881 & 10.0 & 151.0 & 90 & 3.1 & 0.6 & 0.0 & 0.0 & 1.2 & 0.8\end{array}$

$\begin{array}{llllllllllllllll}13 & 242.640 & 36.366 & 242.677 & 36.315 & 6.562 & 10.0 & 149.6 & 90 & 3.2 & 0.6 & 0.0 & 0.0 & 1.1 & 0.8\end{array}$ 
\# Lon-1 Lat-1 Lon-2 Lat-2 L(km) $\mathrm{z}(\mathrm{km})$ strike dip U_S S_S U_d S_d U_t S_t

$\begin{array}{lllllllllllllllll}14 & 242.677 & 36.315 & 242.706 & 36.283 & 4.404 & 10.0 & 143.7 & 90 & 3.3 & 0.6 & 0.0 & 0.0 & 0.8 & 0.8\end{array}$ $\begin{array}{llllllllllllllll}15 & 242.706 & 36.283 & 242.772 & 36.237 & 7.825 & 10.0 & 130.7 & 90 & 3.4 & 0.5 & 0.0 & 0.0 & 0.0 & 0.8\end{array}$

$\begin{array}{llllllllllllllll}16 & 242.772 & 36.237 & 242.797 & 36.195 & 5.174 & 10.0 & 154.2 & 90 & 3.1 & 0.6 & 0.0 & 0.0 & 1.4 & 0.7\end{array}$ $\begin{array}{llllllllllllllll}17 & 242.790 & 36.126 & 242.797 & 36.195 & 7.682 & 10.0 & 4.7 & 90 & 1.9 & 0.7 & 0.0 & 0.0 & 2.8 & 0.5\end{array}$ $\begin{array}{llllllllllllllll}18 & 242.790 & 36.126 & 242.807 & 36.079 & 5.435 & 10.0 & 163.6 & 90 & 2.8 & 0.7 & 0.0 & 0.0 & 1.9 & 0.6\end{array}$ $\begin{array}{llllllllllllllll}19 & 242.793 & 36.041 & 242.807 & 36.079 & 4.401 & 10.0 & 16.7 & 90 & 1.3 & 0.8 & 0.0 & 0.0 & 3.1 & 0.5\end{array}$ $\begin{array}{llllllllllllllll}20 & 242.780 & 35.997 & 242.793 & 36.041 & 5.021 & 10.0 & 13.5 & 90 & 1.5 & 0.8 & 0.0 & 0.0 & 3.1 & 0.5\end{array}$ $\begin{array}{llllllllllllllll}21 & 242.780 & 35.997 & 242.815 & 35.894 & 11.857 & 10.0 & 164.5 & 90 & 2.8 & 0.6 & 0.0 & 0.0 & 2.0 & 0.6\end{array}$ $\begin{array}{llllllllllllllll}22 & 242.815 & 35.894 & 242.862 & 35.795 & 11.777 & 10.0 & 158.9 & 90 & 3.0 & 0.6 & 0.0 & 0.0 & 1.8 & 0.7\end{array}$ $\begin{array}{lllllllllllllll}23 & 242.862 & 35.795 & 242.886 & 35.748 & 5.648 & 10.0 & 157.4 & 90 & 3.0 & 0.6 & 0.0 & 0.0 & 1.7 & 0.7\end{array}$ $\begin{array}{lllllllllllllll}24 & 242.886 & 35.748 & 242.952 & 35.686 & 9.110 & 10.0 & 139.0 & 90 & 3.4 & 0.5 & 0.0 & 0.0 & 0.7 & 0.9\end{array}$ $\begin{array}{lllllllllllllll}25 & 242.952 & 35.686 & 243.070 & 35.639 & 11.889 & 10.0 & 116.0 & 90 & 3.4 & 0.5 & 0.0 & 0.0 & -0.7 & 0.9\end{array}$

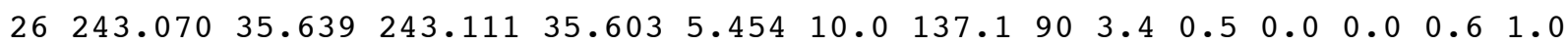

\section{n. Death Valley}

$\begin{array}{lllllllllllllll}1 & 241.620 & 37.951 & 242.060 & 37.483 & 64.832 & 10.0 & 143.1 & 90 & 6.1 & 0.6 & 0.0 & 0.0 & -3.0 & 1.4\end{array}$ $\begin{array}{llllllllllllllll}2 & 242.060 & 37.483 & 242.347 & 37.296 & 32.813 & 10.0 & 129.1 & 90 & 2.1 & 0.8 & 0.0 & 0.0 & -3.6 & 1.3\end{array}$ $\begin{array}{lllllllllllllll}3 & 242.347 & 37.296 & 242.452 & 37.188 & 15.181 & 10.0 & 142.1 & 90 & 2.8 & 0.6 & 0.0 & 0.0 & -2.8 & 1.2\end{array}$ $\begin{array}{llllllllllllllll}4 & 242.452 & 37.188 & 242.557 & 37.080 & 15.189 & 10.0 & 142.1 & 90 & 2.8 & 0.6 & 0.0 & 0.0 & -2.7 & 1.1\end{array}$ $\begin{array}{lllllllllllllllll}5 & 242.557 & 37.080 & 242.593 & 37.054 & 4.310 & 10.0 & 132.0 & 90 & 2.3 & 0.7 & 0.0 & 0.0 & -3.1 & 1.0\end{array}$ $\begin{array}{lllllllllllllllll}6 & 242.593 & 37.054 & 242.687 & 36.960 & 13.372 & 10.0 & 141.2 & 90 & 2.8 & 0.6 & 0.0 & 0.0 & -2.6 & 1.0\end{array}$ $\begin{array}{llllllllllllllll}7 & 242.687 & 36.960 & 242.717 & 36.914 & 5.762 & 10.0 & 152.4 & 90 & 3.2 & 0.5 & 0.0 & 0.0 & -1.9 & 0.9\end{array}$ $\begin{array}{llllllllllllllll}8 & 242.717 & 36.914 & 242.755 & 36.878 & 5.238 & 10.0 & 139.7 & 90 & 2.7 & 0.6 & 0.0 & 0.0 & -2.6 & 0.8\end{array}$ $\begin{array}{lllllllllllllllll}9 & 242.755 & 36.878 & 242.820 & 36.799 & 10.511 & 10.0 & 146.5 & 90 & 3.0 & 0.5 & 0.0 & 0.0 & -2.2 & 0.8\end{array}$

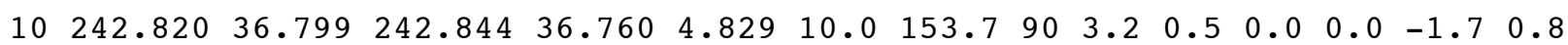
$\begin{array}{lllllllllllllll}11 & 242.844 & 36.760 & 242.900 & 36.709 & 7.553 & 10.0 & 138.5 & 90 & 2.7 & 0.6 & 0.0 & 0.0 & -2.5 & 0.7\end{array}$ $\begin{array}{lllllllllllllll}12 & 242.900 & 36.709 & 243.012 & 36.625 & 13.680 & 10.0 & 132.9 & 90 & 2.4 & 0.6 & 0.0 & 0.0 & -2.6 & 0.6\end{array}$ $\begin{array}{llllllllllllllll}13 & 243.012 & 36.625 & 243.056 & 36.595 & 5.155 & 10.0 & 130.2 & 90 & 2.3 & 0.6 & 0.0 & 0.0 & -2.7 & 0.5\end{array}$ $\begin{array}{llllllllllllllll}14 & 243.056 & 36.595 & 243.108 & 36.563 & 5.854 & 10.0 & 127.3 & 90 & 2.2 & 0.6 & 0.0 & 0.0 & -2.7 & 0.5\end{array}$ $\begin{array}{llllllllllllllll}15 & 243.108 & 36.563 & 243.121 & 36.535 & 3.318 & 10.0 & 159.5 & 90 & 3.3 & 0.5 & 0.0 & 0.0 & -1.1 & 0.6\end{array}$ $\begin{array}{llllllllllllllll}16 & 243.121 & 36.535 & 243.156 & 36.447 & 10.256 & 10.0 & 162.2 & 90 & 3.3 & 0.5 & 0.0 & 0.0 & -0.9 & 0.5\end{array}$ $\begin{array}{llllllllllllllll}17 & 243.156 & 36.447 & 243.173 & 36.398 & 5.647 & 10.0 & 164.3 & 90 & 3.4 & 0.5 & 0.0 & 0.0 & -0.7 & 0.5\end{array}$

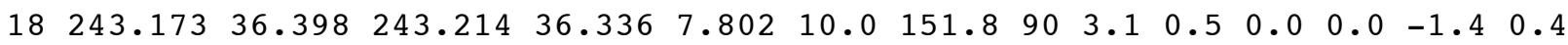
$\begin{array}{llllllllllllllll}19 & 243.214 & 36.336 & 243.229 & 36.301 & 4.111 & 10.0 & 160.9 & 90 & 3.3 & 0.5 & 0.0 & 0.0 & -0.8 & 0.4\end{array}$ $\begin{array}{llllllllllllllll}20 & 243.229 & 36.301 & 243.230 & 36.263 & 4.218 & 10.0 & 178.8 & 90 & 3.4 & 0.4 & 0.0 & 0.0 & 0.2 & 0.4\end{array}$ $\begin{array}{llllllllllllllll}21 & 243.230 & 36.263 & 243.242 & 36.153 & 12.254 & 10.0 & 174.9 & 90 & 3.4 & 0.5 & 0.0 & 0.0 & 0.1 & 0.4\end{array}$ $\begin{array}{lllllllllllllllll}22 & 243.242 & 36.153 & 243.243 & 36.054 & 10.986 & 10.0 & 179.5 & 90 & 3.4 & 0.4 & 0.0 & 0.0 & 0.5 & 0.3\end{array}$ $\begin{array}{llllllllllllllll}23 & 243.243 & 36.054 & 243.276 & 36.003 & 6.393 & 10.0 & 152.3 & 90 & 3.2 & 0.5 & 0.0 & 0.0 & -1.1 & 0.2\end{array}$ $\begin{array}{lllllllllllllll}24 & 243.276 & 36.003 & 243.292 & 35.926 & 8.665 & 10.0 & 170.4 & 90 & 3.4 & 0.5 & 0.0 & 0.0 & 0.0 & 0.1\end{array}$ $\begin{array}{lllllllllllllllll}25 & 243.292 & 35.926 & 243.336 & 35.877 & 6.733 & 10.0 & 143.8 & 90 & 3.1 & 0.4 & 0.0 & 0.0 & -1.4 & 0.2\end{array}$ $\begin{array}{llllllllllllllll}26 & 243.336 & 35.877 & 243.384 & 35.846 & 5.534 & 10.0 & 128.4 & 90 & 2.6 & 0.4 & 0.0 & 0.0 & -2.1 & 0.3\end{array}$ $\begin{array}{llllllllllllllll}27 & 243.384 & 35.846 & 243.399 & 35.791 & 6.251 & 10.0 & 167.5 & 90 & 3.3 & 0.5 & 0.0 & 0.0 & -0.0 & 0.0\end{array}$ $\begin{array}{lllllllllllllll}28 & 243.399 & 35.791 & 243.445 & 35.723 & 8.616 & 10.0 & 151.1 & 90 & 3.2 & 0.5 & 0.0 & 0.0 & -0.9 & 0.2\end{array}$ $\begin{array}{lllllllllllllll}29 & 243.445 & 35.723 & 243.484 & 35.703 & 4.169 & 10.0 & 122.2 & 90 & 2.4 & 0.3 & 0.0 & 0.0 & -2.3 & 0.4\end{array}$ $\begin{array}{lllllllllllllllll}30 & 243.484 & 35.703 & 243.516 & 35.675 & 4.248 & 10.0 & 137.0 & 90 & 2.9 & 0.4 & 0.0 & 0.0 & -1.6 & 0.3\end{array}$ $\begin{array}{lllllllllllllllll}31 & 243.516 & 35.675 & 243.551 & 35.627 & 6.198 & 10.0 & 149.2 & 90 & 3.2 & 0.5 & 0.0 & 0.0 & -0.9 & 0.3\end{array}$ $\begin{array}{lllllllllllllll}32 & 243.551 & 35.627 & 243.601 & 35.603 & 5.255 & 10.0 & 120.4 & 90 & 2.4 & 0.3 & 0.0 & 0.0 & -2.2 & 0.5\end{array}$ $\begin{array}{llllllllllllllll}33 & 243.601 & 35.603 & 243.608 & 35.596 & 1.003 & 10.0 & 140.8 & 90 & 3.0 & 0.4 & 0.0 & 0.0 & -1.3 & 0.4\end{array}$ 
Table DR2. Nominally interseismic GPS velocity field used to constrain the block model. Columns give: station name, longitude, latitude, east velocity, north velocity, east uncertainty, north uncertainty

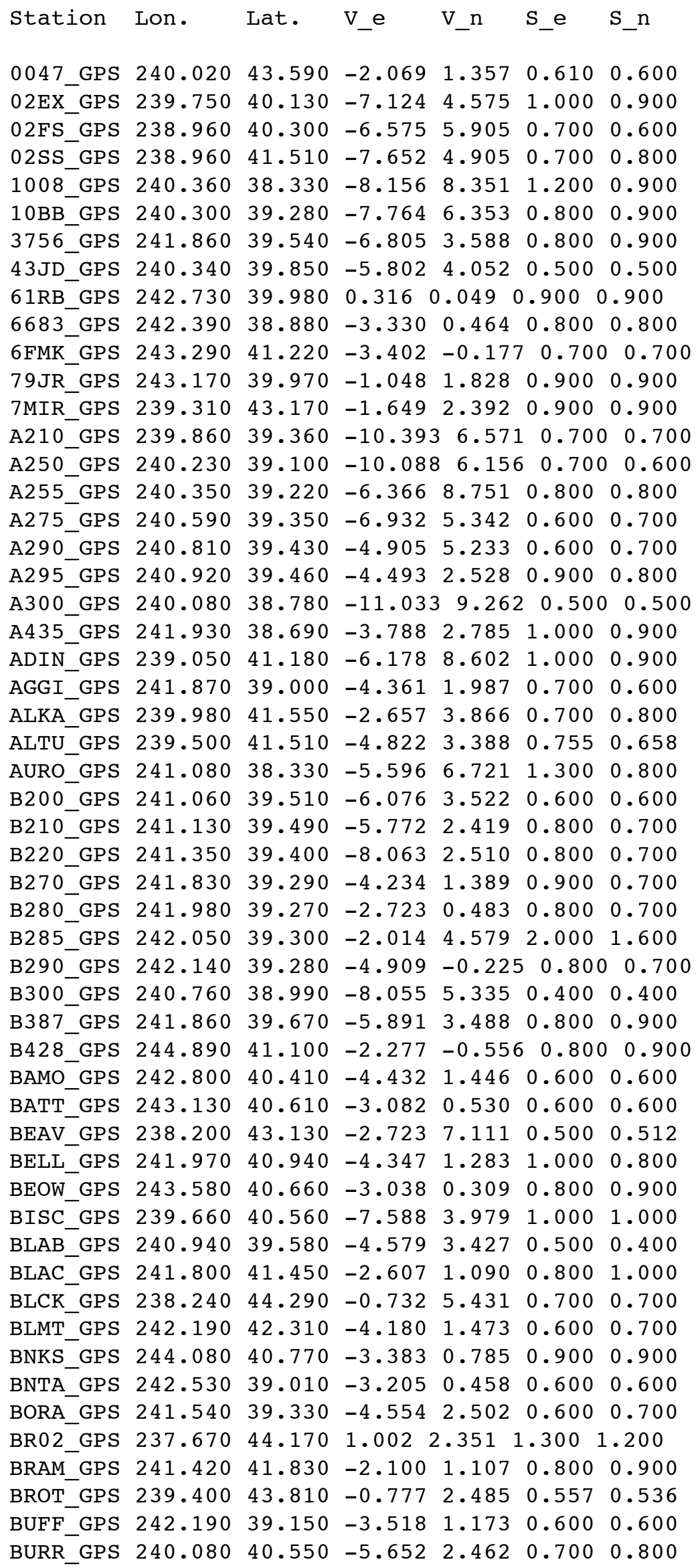


BUTN_GPS 242.430 41.010 $-1.999-0.538 \quad 0.800 \quad 0.900$ $\begin{array}{lllllll}\mathrm{BX} 46 \text { GPS } & 241.690 & 39.270 & -4.848 & 2.395 & 0.900 & 0.600\end{array}$ C200_GPS 242.290 39.260 $-2.498-0.0310 .600 \quad 0.600$ $\begin{array}{lllllll}C 220 & \text { GPS } 242.590 & 39.360 & -2.462 & 0.455 & 1.200 & 0.900\end{array}$ $\begin{array}{llllllll}C 240 & \text { GPS } 242.860 & 39.490 & -2.426 & 0.043 & 0.600 & 0.600\end{array}$ $\begin{array}{llllllll}C 260 & \text { GPS } 243.060 & 39.400 & -2.819 & 0.734 & 0.900 & 0.600\end{array}$ C280_GPS $243.380 \quad 39.480-3.183-0.982 \quad 0.800 \quad 0.600$ C300_GPS 242.120 38.760 $\quad-3.965 \quad 1.276 \quad 0.400 \quad 0.400$ C344_GPS 242.200 40.910 -2.230 -1.027 $1.300 \quad 0.900$ C753_GPS 239.380 44.310 $-0.723 \quad 2.289 \quad 1.000 \quad 1.100$ CARL_GPS $243.840 \quad 40.710 \quad-2.010 \quad 1.196 \quad 1.200 \quad 1.100$ CAST_GPS $249.320 \quad 39.190 \quad-0.772 \quad-0.055 \quad 0.356 \quad 0.338$ CEDA_GPS $247.140 \quad 40.680 \quad-3.301-0.529 \quad 0.316 \quad 0.314$ CEDR_GPS 239.740 $41.550 \quad-5.379 \quad 4.076 \quad 0.900 \quad 0.900$ CHIL GPS $240.800 \quad 39.360 \quad-5.413 \quad 2.833 \quad 0.500 \quad 0.500$ $\begin{array}{llllllll}\text { CHLK_GPS } 240.120 & 39.510 & -9.456 & 3.861 & 0.500 & 0.500\end{array}$ $\begin{array}{llllllll}\text { CHO1_GPS } & 238.340 & 39.430 & -11.038 & 7.049 & 0.436 & 0.433\end{array}$ CMBB_GPS $239.610 \quad 38.030 \quad-11.429 \quad 7.847 \quad 0.386 \quad 0.383$ CNBY GPS 239.130 $41.430 \quad-5.746 \quad 5.299 \quad 0.700 \quad 0.800$ CNWY_GPS $240.820 \quad 38.080 \quad-6.943 \quad 9.832 \quad 1.000 \quad 0.800$ COON_GPS $247.880 \quad 40.650 \quad-2.455-0.515 \quad 0.337 \quad 0.353$ CRTS_GPS $244.990 \quad 40.660 \quad-2.218 \quad-0.961 \quad 0.600 \quad 0.600$ CTNP_GPS $240.530 \quad 41.900 \quad-2.272 \quad 2.344 \quad 0.800 \quad 1.000$ CUPO_GPS $237.980 \quad 44.190 \quad-1.367 \quad 6.840 \quad 1.000 \quad 0.900$ CUSG_GPS $242.960 \quad 40.000 \quad-2.363-1.062 \quad 0.700 \quad 0.700$ D100_GPS 243.840 40.080 $-3.880-0.404 \quad 0.400 \quad 0.400$ D200 GPS $243.690 \quad 39.530-3.052 \quad-0.396 \quad 0.600 \quad 0.500$ D220 GPS $244.020 \quad 39.540 \quad-6.323-0.612 \quad 1.000 \quad 0.700$ D240_GPS 244.200 39.380 $-4.026-1.321 \quad 1.000 \quad 0.600$ D260_GPS $244.440 \quad 39.400 \quad-3.304 \quad-0.633 \quad 0.800 \quad 0.600$ D280 GPS $244.920 \quad 39.420 \quad-3.662 \quad-2.257 \quad 0.800 \quad 0.700$ D300_GPS 243.250 39.060 $-4.239-0.975 \quad 0.500 \quad 0.500$ DECH_GPS $240.910 \quad 38.050 \quad-11.139 \quad 8.428 \quad 0.500 \quad 0.500$ DORF_GPS 239.060 39.820 $\quad-8.215 \quad 4.801 \quad 1.200 \quad 1.000$ DRYX_GPS $238.910 \quad 44.210 \quad 1.523 \quad 6.907 \quad 0.800 \quad 0.800$ DYER_GPS $241.960 \quad 37.740 \quad-5.202 \quad 3.002 \quad 0.444 \quad 0.442$ E100_GPS 245.350 39.900 -4.173 $-0.379 \quad 0.500 \quad 0.500$ E200_GPS $245.050 \quad 39.290 \quad-3.866 \quad 0.036 \quad 0.500 \quad 0.500$ E220_GPS $245.340 \quad 39.030-4.671-0.479 \quad 0.800 \quad 0.600$ E240_GPS $245.550 \quad 39.070-1.349 \quad-0.190 \quad 0.800 \quad 0.600$ E259_GPS 245.760 39.100 $-3.129-1.101 \quad 1.200 \quad 0.800$ E280_GPS $246.110 \quad 39.040 \quad-4.807 \quad-2.419 \quad 1.000 \quad 0.700$ E300_GPS $244.870 \quad 38.890 \quad-3.825 \quad-0.855 \quad 0.500 \quad 0.500$ E843 GPS 239.390 40.450 $\quad-6.223 \quad 4.089 \quad 1.100 \quad 0.900$ EARN_GPS $240.520 \quad 39.550 \quad-8.617 \quad 3.844 \quad 0.400 \quad 0.400$ EGAN_GPS 245.060 39.350 $-3.058-0.564 \quad 0.400 \quad 0.400$ ELKO_GPS $244.180 \quad 40.910 \quad-4.159 \quad-0.020 \quad 0.400 \quad 0.400$ F067_GPS $240.950 \quad 41.880-3.636-0.973 \quad 0.8001 .000$ F091_GPS 239.460 43.390 -0.312 3.786 $0.900 \quad 1.000$ F100_GPS 246.420 39.550 -2.224 $-1.436 \quad 0.500 \quad 0.500$ F200_GPS $246.370 \quad 39.080-3.881-0.733 \quad 0.900 \quad 0.700$ F220 GPS $246.620 \quad 39.020-4.068 \quad-0.247 \quad 1.000 \quad 0.700$ F250_GPS $246.950 \quad 39.280-1.811-1.765 \quad 0.900 \quad 0.600$ F270_GPS $247.160 \quad 39.320 \quad-2.190 \quad 0.323 \quad 0.900 \quad 0.700$ F279_GPS $241.850 \quad 39.230 \quad-3.438 \quad 1.388 \quad 0.600 \quad 0.600$ F280 GPS $247.450 \quad 39.370-1.860 \quad 0.307 \quad 1.000 \quad 0.700$ F300_GPS $246.330 \quad 38.790 \quad-2.917 \quad-0.331 \quad 0.500 \quad 0.500$ FLRN GPS $240.800 \quad 39.940 \quad-5.853 \quad 2.733 \quad 0.400 \quad 0.400$ FLS2_GPS $241.570 \quad 37.710 \quad-7.419 \quad 6.500 \quad 1.200 \quad 1.100$ FOOT_GPS $246.190 \quad 39.370-3.461-0.307 \quad 0.338 \quad 0.320$ 
FRAZ GPS 238.710 41.100 $\quad$-9.916 $7.514 \quad 0.900 \quad 0.900$ FUSG GPS $239.820 \quad 39.920 \quad-8.339 \quad 3.872 \quad 1.200 \quad 0.900$ G101_GPS $247.850 \quad 39.600-3.501-0.016 \quad 0.800 \quad 0.700$ G200_GPS $247.760 \quad 39.140 \quad-2.662-0.611 \quad 0.800 \quad 0.600$ G220_GPS $247.960 \quad 39.080-5.753-0.422 \quad 0.700 \quad 0.700$ G250 GPS $248.260 \quad 38.900 \quad-5.250 \quad 0.960 \quad 0.700 \quad 0.600$ G298 GPS $241.810 \quad 40.120 \quad-5.148 \quad 1.190 \quad 0.900 \quad 0.900$ G300_GPS $247.410 \quad 38.600-3.352-0.591 \quad 0.500 \quad 0.500$ GABB_GPS $242.080 \quad 38.970 \quad-4.238 \quad 1.229 \quad 0.444 \quad 0.442$ GARL_GPS $240.640 \quad 40.420 \quad-5.417 \quad 2.740 \quad 0.400 \quad 0.400$ GOLC_GPS $242.530 \quad 40.940 \quad-2.698 \quad 0.658 \quad 0.900 \quad 0.900$ GOSH_GPS $245.820 \quad 40.640 \quad-3.625 \quad-0.368 \quad 0.318 \quad 0.316$ GUAN_GPS $240.520 \quad 42.020 \quad-4.260 \quad 1.844 \quad 0.900 \quad 1.000$ H100 GPS $248.980 \quad 39.290 \quad-0.746 \quad-0.782 \quad 0.500 \quad 0.500$ H112_GPS 239.820 $38.630 \quad-10.370 \quad 8.872 \quad 0.700 \quad 0.800$ H130_GPS $240.920 \quad 38.160 \quad-11.227 \quad 7.128 \quad 1.100 \quad 0.900$ H200 GPS $248.890 \quad 38.830 \quad-2.108-0.177 \quad 0.900 \quad 0.600$

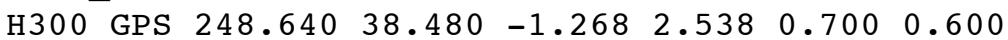
HEBE_GPS $248.630 \quad 40.510-0.934 \quad-0.239 \quad 0.335 \quad 0.333$ HELI_GPS $238.980 \quad 39.730 \quad-7.631 \quad 4.604 \quad 1.200 \quad 0.900$ HELO_GPS $244.380 \quad 40.950-3.338-1.730 \quad 0.900 \quad 1.000$ HHTT_GPS $238.260 \quad 40.870 \quad-8.680 \quad 6.231 \quad 1.000 \quad 0.900$ HICK_GPS $241.180 \quad 38.280 \quad-6.693 \quad 7.117 \quad 1.000 \quad 1.000$ HILD_GPS $238.490 \quad 42.250-1.819 \quad 5.122 \quad 0.800 \quad 1.000$ HLNB_GPS $238.730 \quad 41.480 \quad-4.676 \quad 2.514 \quad 0.400 \quad 0.400$ HORU_GPS $241.190 \quad 37.980 \quad-9.723 \quad 10.317 \quad 0.800 \quad 0.800$ HOTC GPS $239.300 \quad 41.490 \quad-2.624 \quad 3.292 \quad 0.900 \quad 0.900$ HP26_GPS $240.010 \quad 40.240 \quad-5.730 \quad 3.482 \quad 0.680 \quad 0.652$ HSPR_GPS $240.350 \quad 40.920 \quad-3.890 \quad 3.151 \quad 0.900 \quad 0.800$ HSTN_GPS $239.070 \quad 44.260 \quad 0.043 \quad 3.001 \quad 0.800 \quad 0.800$ HUSG_GPS $239.500 \quad 40.630 \quad-5.295 \quad 5.385 \quad 0.800 \quad 0.700$ HYAT_GPS $237.500 \quad 42.150 \quad-1.719 \quad 5.857 \quad 1.100 \quad 1.100$ J288_GPS $238.650 \quad 42.410-1.388 \quad 2.917 \quad 0.800 \quad 1.000$ J74X_GPS $241.750 \quad 40.940 \quad-4.666 \quad 0.093 \quad 1.500 \quad 0.900$ J789_GPS $238.170 \quad 42.870 \quad-1.785 \quad 4.834 \quad 0.900 \quad 1.000$ JNCT_GPS $240.530 \quad 38.360 \quad-6.438 \quad 8.744 \quad 1.000 \quad 0.700$ JUNI_GPS $240.070 \quad 42.930 \quad-1.952 \quad 2.852 \quad 0.507 \quad 0.510$ K102_GPS $242.320 \quad 40.160-3.700-0.433 \quad 0.900 \quad 0.900$ K589_GPS $240.150 \quad 42.120 \quad-2.583 \quad 0.159 \quad 1.000 \quad 1.000$ L091_GPS $239.360 \quad 43.290 \quad-1.332 \quad 2.690 \quad 0.900 \quad 1.000$ LEWI_GPS $243.140 \quad 40.400 \quad-2.869 \quad-0.692 \quad 0.342 \quad 0.325$ LIZZ_GPS $241.770 \quad 38.530 \quad-3.718 \quad 3.292 \quad 0.600 \quad 0.600$ LL92_GPS $243.020 \quad 40.360 \quad-3.319 \quad 0.435 \quad 0.600 \quad 0.700$ LOLA_GPS $241.440 \quad 40.070 \quad-6.185 \quad 2.106 \quad 0.600 \quad 0.600$ LOOP_GPS $241.010 \quad 42.720 \quad-3.442 \quad 0.524 \quad 0.800 \quad 0.700$ LOWE_GPS $242.360 \quad 40.030 \quad-4.410 \quad 0.266 \quad 0.600 \quad 0.600$ LUCK_GPS $241.230 \quad 38.420 \quad-6.774 \quad 6.315 \quad 1.100 \quad 0.800$ LUMP_GPS $238.850 \quad 39.630 \quad-7.552 \quad 6.409 \quad 1.400 \quad 0.900$ M504_GPS $238.470 \quad 41.720 \quad-5.975 \quad 4.723 \quad 0.500 \quad 0.500$ M753_GPS 239.580 44.400 $0.406 \quad 3.082 \quad 1.000 \quad 1.300$ MAGU_GPS $240.210 \quad 39.370 \quad-9.562 \quad 6.057 \quad 0.400 \quad 0.400$ MCAR_GPS 238.600 41.050 -10.131 3.818 1.000 1.000 MCOY_GPS 242.400 40.110 $\quad-4.799 \quad 1.064 \quad 0.600 \quad 0.600$ MICK_GPS $241.580 \quad 42.660 \quad-2.997 \quad 1.500 \quad 0.600 \quad 0.600$ MINE_GPS $243.900 \quad 40.150 \quad-3.569 \quad 0.286 \quad 0.343 \quad 0.343$ MN54_GPS $241.940 \quad 39.200 \quad-4.734 \quad 1.584 \quad 0.600 \quad 0.600$ MOND GPS $241.720 \quad 39.400 \quad-5.531 \quad 1.594 \quad 0.700 \quad 0.700$ MONI_GPS $243.280 \quad 39.150 \quad-3.727 \quad 0.223 \quad 0.400 \quad 0.400$ MOOR_GPS 245.200 41.110 -2.650 -1.972 1.0001 .000 MUSB_GPS $240.690 \quad 37.170-11.115 \quad 8.876 \quad 0.379 \quad 0.393$ 
N067 GPS $241.150 \quad 41.890 \quad-2.418 \quad 2.718 \quad 0.800 \quad 0.900$ N843 GPS 239.330 $40.420 \quad-6.131 \quad 4.191 \quad 1.400 \quad 0.900$ $\begin{array}{lllllllll}\text { NEWS_GPS } & 242.490 & 39.690 & -4.490 & 0.467 & 0.481 & 0.487\end{array}$ OAKR_GPS $237.500 \quad 43.750 \quad 0.262 \quad 5.769 \quad 0.507 \quad 0.487$ OBSD_GPS $240.760 \quad 41.880 \quad-1.653 \quad 1.735 \quad 0.700 \quad 0.800$ OREG_GPS $239.410 \quad 42.200 \quad-3.441 \quad 2.688 \quad 0.900 \quad 1.000$ ORVB_GPS $238.500 \quad 39.550 \quad-10.790 \quad 7.022 \quad 0.400 \quad 0.400$ OVRP_GPS $238.720 \quad 41.710 \quad-6.154 \quad 3.614 \quad 0.400 \quad 0.400$ P19A_GPS $240.530 \quad 40.580 \quad-2.810 \quad 2.144 \quad 0.900 \quad 0.900$ P208_GPS $240.080 \quad 39.110 \quad-9.800 \quad 6.362 \quad 0.500 \quad 0.500$ P515_GPS $240.220 \quad 41.000 \quad-5.693 \quad 2.657 \quad 0.700 \quad 0.800$ PARA_GPS $242.310 \quad 41.280 \quad-2.281 \quad 0.268 \quad 0.900 \quad 0.900$ PARS_GPS $239.930 \quad 42.210 \quad-2.793 \quad 2.468 \quad 0.700 \quad 0.700$ PERS GPS $241.250 \quad 40.860 \quad-7.218 \quad 0.514 \quad 1.200 \quad 0.900$ PIBU_GPS $238.720 \quad 44.060 \quad 0.260 \quad 3.327 \quad 0.602 \quad 0.585$ PITR_GPS 238.460 40.980 $\quad-4.1519 .623 \quad 1.000 \quad 1.000$ PL20 GPS $239.280 \quad 42.260 \quad-3.447 \quad 3.993 \quad 0.900 \quad 0.900$ POST_GPS $241.960 \quad 39.040 \quad-4.249 \quad 2.883 \quad 0.600 \quad 0.600$ Q837_GPS 239.430 40.550 $\quad-7.809 \quad 3.588 \quad 1.000 \quad 0.900$ QUIN_GPS $239.060 \quad 39.970 \quad-9.600 \quad 6.301 \quad 0.400 \quad 0.400$ R090_GPS $239.880 \quad 43.480 \quad 0.036 \quad 3.570 \quad 0.900 \quad 0.900$ RAIL_GPS $244.340 \quad 38.280 \quad-3.817 \quad 0.209 \quad 0.444 \quad 0.442$ RATT_GPS $241.300 \quad 40.000 \quad-6.604 \quad 2.512 \quad 0.500 \quad 0.500$ ROCO_GPS $238.900 \quad 42.430 \quad-0.963 \quad 1.607 \quad 1.100 \quad 1.000$ ROUN_GPS $238.540 \quad 41.430 \quad-5.598 \quad 3.621 \quad 0.400 \quad 0.400$ RUBY_GPS $244.880 \quad 40.620 \quad-3.634 \quad-0.207 \quad 0.335 \quad 0.333$ RUST GPS $237.650 \quad 42.620 \quad-2.299 \quad 6.336 \quad 0.616 \quad 0.598$ SAGE GPS $239.960 \quad 39.790 \quad-8.841 \quad 4.867 \quad 0.500 \quad 0.500$ SHEL_GPS $241.680 \quad 39.040 \quad-5.372 \quad 1.096 \quad 0.900 \quad 0.700$ SHIN_GPS $239.770 \quad 40.590 \quad-6.749 \quad 4.175 \quad 0.3350 .333$ $\begin{array}{llllllll}\text { SHLD GPS } 240.980 & 41.870 & -3.519 & 2.599 & 0.346 & 0.346\end{array}$ SHON_GPS $242.810 \quad 40.030 \quad-5.472 \quad 1.045 \quad 0.700 \quad 0.800$ SILV_GPS $238.940 \quad 43.120 \quad-1.688 \quad 4.606 \quad 0.600 \quad 0.600$ SIST_GPS $238.440 \quad 44.310 \quad 0.924 \quad 3.862 \quad 0.520 \quad 0.527$ SLID_GPS $240.120 \quad 39.310 \quad-9.908 \quad 5.598 \quad 0.473 \quad 0.411$ SLR1_GPS $241.790 \quad 39.110 \quad-6.256 \quad 3.391 \quad 0.600 \quad 0.600$ SMEL_GPS $247.160 \quad 39.430 \quad-3.077 \quad-0.366 \quad 0.320 \quad 0.320$ SMOK_GPS $240.350 \quad 40.620 \quad-6.061 \quad 3.710 \quad 0.719 \quad 0.618$ SNDS_GPS $241.580 \quad 39.150 \quad-7.769 \quad 3.300 \quad 0.600 \quad 0.700$ SODH_GPS $241.980 \quad 41.410 \quad-2.096 \quad 2.183 \quad 0.600 \quad 0.700$ STEA_GPS $237.260 \quad 43.340 \quad-1.341 \quad 6.357 \quad 0.487 \quad 0.495$ SUTB_GPS $238.180 \quad 39.210 \quad-10.871 \quad 7.506 \quad 0.370 \quad 0.353$ T128_GPS $242.760 \quad 42.930-2.761-0.853 \quad 0.900 \quad 1.000$ TMBR_GPS 238.700 $41.630 \quad-4.664 \quad 5.615 \quad 0.400 \quad 0.400$ TONO_GPS $242.820 \quad 38.100 \quad-4.222 \quad 0.772 \quad 0.348 \quad 0.330$ TRGO_GPS $240.870 \quad 40.770 \quad-7.461 \quad 2.530 \quad 1.000 \quad 0.800$ TUFF_GPS 238.790 42.440 $\quad-2.272 \quad 1.111 \quad 1.000 \quad 1.000$ TUNG_GPS $241.740 \quad 40.400 \quad-5.591 \quad 2.009 \quad 0.335 \quad 0.335$ U698_GPS $241.700 \quad 43.170-2.030-1.405 \quad 0.9001 .000$ UPSA_GPS $241.200 \quad 39.630 \quad-6.647 \quad 3.055 \quad 0.342 \quad 0.323$ UU83_GPS $239.680 \quad 39.320 \quad-9.1126 .778 \quad 0.700 \quad 0.600$ V209_GPS $240.460 \quad 39.080 \quad-6.671 \quad 4.847 \quad 0.600 \quad 0.500$ VALM_GPS $242.890 \quad 40.780 \quad-6.384 \quad 0.241 \quad 0.800 \quad 0.900$ VIDA_GPS $237.430 \quad 44.150 \quad 1.349 \quad 4.769 \quad 0.594 \quad 0.545$ W072_GPS 239.230 $43.010 \quad-3.527 \quad 3.277 \quad 0.628 \quad 0.600$ W078_GPS $241.420 \quad 38.610 \quad-5.039 \quad 4.707 \quad 1.100 \quad 0.900$ W67R_GPS $239.640 \quad 42.190 \quad-4.621 \quad 2.879 \quad 0.700 \quad 0.800$ W784_GPS $237.990 \quad 42.140 \quad-1.776 \quad 2.040 \quad 1.000 \quad 1.000$ WICK_GPS $238.310 \quad 43.680 \quad-3.170 \quad 6.335 \quad 0.492 \quad 0.500$ WILD_GPS $241.630 \quad 40.020 \quad-5.374 \quad 4.098 \quad 0.700 \quad 0.700$ 
WILO GPS $243.590 \quad 41.210 \quad-2.277 \quad-0.792 \quad 0.600 \quad 0.700$ $\begin{array}{lllllll}X 360 \text { GPS } & 241.610 & 38.540 & -3.030 & 2.899 & 1.000 & 0.900\end{array}$ Y090_GPS $239.690 \quad 43.430 \quad-0.187 \quad 2.977 \quad 0.800 \quad 0.900$ $\begin{array}{llllllll}\text { YBHB GPS } & 237.290 & 41.730 & -3.575 & 6.787 & 0.424 & 0.421\end{array}$

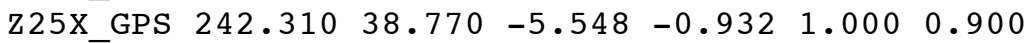
KELY GPS $309.050 \quad 66.990 \quad 0.569 \quad-0.543 \quad 0.363 \quad 0.369$ STJO_GPS $307.320 \quad 47.600 \quad-1.395 \quad-0.657 \quad 0.800 \quad 0.100$ $\begin{array}{llllllll}\text { BRMU_GPS } & 295.300 & 32.310 & 0.117 & -0.249 & 0.700 & 0.700\end{array}$ THU1_GPS $291.210 \quad 76.540 \quad-1.933 \quad-0.805 \quad 0.548 \quad 0.548$ WES2_GPS $288.510 \quad 42.610 \quad 0.461 \quad-1.144 \quad 0.418 \quad 0.409$ $\begin{array}{lllllll}\text { NLIB_GPS } & 281.930 & 45.960 & 0.475 & -0.605 & 0.360 & 0.360\end{array}$ $\begin{array}{lllllll}\text { ALGO_GPS } & 268.420 & 41.770 & 0.102 & 0.021 & 0.332 & 0.321\end{array}$ $\begin{array}{lllllll}\text { YELL_GPS } & 245.520 & 62.480 & -0.434 & -0.312 & 0.330 & 0.347\end{array}$ $\begin{array}{lllllll}\text { DRAO GPS } & 240.380 & 49.320 & 1.065 & 1.366 & 0.353 & 0.361\end{array}$ FAIR_GPS $212.500 \quad 64.980 \quad 0.464 \quad-3.506 \quad 0.365 \quad 0.354$ BILI_GPS $166.440 \quad 68.080 \quad 2.113 \quad-1.869 \quad 0.671 \quad 0.689$ $\begin{array}{lllllll}\text { PETP_GPS } & 158.610 & 53.010 & -5.856 & 10.623 & 1.600 & 1.600\end{array}$ MAGO_GPS $150.770 \quad 59.580 \quad 4.682 \quad-5.544 \quad 1.500 \quad 1.600$ YSSK_GPS $142.720 \quad 47.030 \quad 8.391 \quad 0.490 \quad 1.700 \quad 1.700$ TIXI_GPS $128.870 \quad 71.630 \quad-2.546 \quad-1.133 \quad 1.500 \quad 1.500$ 0047 GPS $240.017 \quad 43.590 \quad-2.069 \quad 1.357 \quad 0.610 \quad 0.600$ 0113_GPS $235.842 \quad 41.933 \quad 1.899 \quad 11.340 \quad 0.743 \quad 0.728$ 0209_GPS $238.742 \quad 41.671 \quad-2.440 \quad 4.050 \quad 1.5801 .610$ 0226_GPS $240.006 \quad 40.245 \quad-5.730 \quad 3.482 \quad 0.680 \quad 0.652$ 036C_GPS $244.168 \quad 41.511 \quad-2.840 \quad-0.620 \quad 0.800 \quad 0.750$ 1214_GPS $237.059 \quad 42.397 \quad-0.300 \quad 6.590 \quad 1.750 \quad 1.010$ $\begin{array}{lllllll}16 \mathrm{EM} \text { GPS } & 243.534 & 42.656 & -2.380 & 1.810 & 0.860 & 0.840\end{array}$ 1882 GPS $239.335 \quad 47.768 \quad 1.730 \quad 2.530 \quad 0.520 \quad 0.550$ 217U_GPS $238.095 \quad 46.542 \quad 2.740 \quad 5.030 \quad 0.480 \quad 0.590$ 2780 GPS $235.676 \quad 43.344 \quad 5.080 \quad 11.270 \quad 0.580 \quad 0.450$ 4S9B GPS $237.409 \quad 45.216 \quad 1.670 \quad 6.896 \quad 1.015 \quad 0.865$ 4Z9A_GPS $236.549 \quad 48.124 \quad 5.930 \quad 4.860 \quad 0.730 \quad 0.920$ 74 LR_GPS $240.581 \quad 41.173 \quad-4.190 \quad 2.250 \quad 0.700 \quad 0.730$ A074_GPS 238.825 $43.313 \quad-1.550 \quad 4.450 \quad 0.700 \quad 0.690$ A16N_GPS $235.940 \quad 44.587 \quad 6.729 \quad 9.813 \quad 0.442 \quad 0.409$ A479_GPS $236.467 \quad 46.991 \quad 7.100 \quad 6.100 \quad 0.530 \quad 0.600$ A515_GPS $239.575 \quad 48.594 \quad 0.700 \quad 1.770 \quad 0.950 \quad 0.920$ A545_GPS $239.256 \quad 45.475 \quad 0.420 \quad 3.060 \quad 0.600 \quad 0.620$ A699_GPS $241.549 \quad 43.216 \quad-2.620 \quad 1.230 \quad 0.730 \quad 0.720$ ACME_GPS $237.796 \quad 48.711 \quad 3.290 \quad 4.280 \quad 0.790 \quad 1.010$ AHID_GPS $248.936 \quad 42.773 \quad-0.893 \quad-0.967 \quad 0.458 \quad 0.430$ AIRP_GPS $242.185 \quad 44.833 \quad-0.690 \quad 0.350 \quad 0.560 \quad 0.500$ $\begin{array}{lllllll}\text { ALAM_GPS } & 244.842 & 37.358 & -3.280 & -0.160 & 0.390 & 0.380\end{array}$ $\begin{array}{lllllll}A L C C \text { GPS } & 232.481 & 50.458 & -0.190 & 3.010 & 0.730 & 0.750\end{array}$ ALEX_GPS $234.507 \quad 49.738 \quad 5.280 \quad 5.960 \quad 1.020 \quad 1.050$ ALKA_GPS $240.001 \quad 42.954 \quad-2.480 \quad 3.560 \quad 0.620 \quad 0.700$ ALTA_GPS $238.253 \quad 42.209 \quad-3.430 \quad 5.150 \quad 0.400 \quad 0.440$ $\begin{array}{lllllll}\text { ANAW_GPS } & 233.453 & 49.789 & 5.880 & 5.890 & 0.740 & 0.780\end{array}$ $\begin{array}{llllllll}\text { ANNA_GPS } & 234.685 & 50.491 & 2.350 & 3.110 & 0.850 & 0.880\end{array}$ $\begin{array}{lllllll}\text { APEX_GLA } & 245.068 & 36.319 & -3.390 & -0.290 & 0.380 & 0.370\end{array}$ APSA_GPS $237.015 \quad 46.671 \quad 3.770 \quad 5.740 \quad 0.400 \quad 0.400$ ARGU_GLA $242.478 \quad 36.050 \quad-7.280 \quad 5.840 \quad 0.400 \quad 0.390$

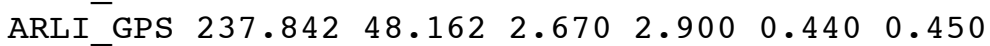
ARLO_GPS $237.846 \quad 48.170 \quad 2.850 \quad 3.640 \quad 0.590 \quad 0.680$ ARP0_GPS $237.193 \quad 46.477 \quad 8.840 \quad 3.690 \quad 1.450 \quad 1.670$ ASHO_GPS $237.293 \quad 42.216 \quad-5.030 \quad 7.500 \quad 0.680 \quad 0.750$ ASTO_GPS $236.168 \quad 46.173 \quad 7.280 \quad 8.290 \quad 0.760 \quad 0.780$ ATHE_GPS $241.529 \quad 45.813 \quad-0.240 \quad 0.450 \quad 0.520 \quad 0.510$ ATKI_GPS $236.747 \quad 49.337 \quad 3.110 \quad 3.500 \quad 1.170 \quad 0.930$ AVA2_GPS $237.751 \quad 47.686 \quad 4.250 \quad 3.180 \quad 0.580 \quad 0.580$ 


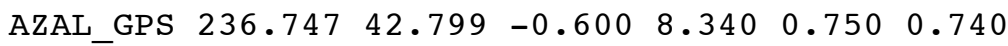
$\begin{array}{llllllll}B 059 & \text { GPS } & 238.574 & 47.735 & 3.630 & 3.100 & 0.540 & 0.560\end{array}$ B073_GPS $241.271 \quad 40.848 \quad-4.540 \quad 2.720 \quad 0.880 \quad 0.910$ B197_GPS 237.245 47.501 $4.920 \quad 5.580 \quad 0.580 \quad 0.640$ B317 GPS $240.273 \quad 46.578 \quad 0.720 \quad 1.710 \quad 0.550 \quad 0.610$ B737 GPS $236.803 \quad 44.149 \quad 2.420 \quad 5.000 \quad 0.910 \quad 0.990$ B741_GPS $236.589 \quad 43.129 \quad-0.680 \quad 8.530 \quad 0.660 \quad 0.720$ BAKR_GPS $238.326 \quad 48.865 \quad 1.550 \quad 1.990 \quad 1.240 \quad 1.390$ BAL2_GPS 235.934 $44.830 \quad 7.390 \quad 9.810 \quad 0.570 \quad 0.590$ BALL_GPS $235.842 \quad 49.347 \quad 4.770 \quad 5.270 \quad 0.550 \quad 0.600$ BAMF_GPS $234.866 \quad 48.828 \quad 8.600 \quad 7.460 \quad 0.780 \quad 0.960$ BATT_GPS $237.453 \quad 45.781 \quad 2.960 \quad 5.760 \quad 0.420 \quad 0.450$ BCHD_GPS $236.34948 .316 \quad 5.530 \quad 4.820 \quad 1.310 \quad 0.930$ BCHR GPS 234.777 $49.650 \quad 5.800 \quad 4.600 \quad 0.720 \quad 0.780$ BCOV GPS 233.157 50.544 2.790 $4.200 \quad 0.690 \quad 0.710$ BEAV_GPS 238.195 43.133 $\quad-2.723 \quad 7.111 \quad 0.500 \quad 0.512$ BHAP_GPS $237.465 \quad 48.791 \quad 3.830 \quad 2.090 \quad 1.950 \quad 1.140$ BIGC_GPS $236.41946 .146 \quad 4.740 \quad 6.430 \quad 0.540 \quad 0.600$ BIGH_GPS $241.470 \quad 45.600 \quad-0.370 \quad 1.030 \quad 1.080 \quad 0.920$ BLCO_GPS 235.514 $42.801 \quad 3.530 \quad 12.790 \quad 0.430 \quad 0.420$ BLK4_GPS $237.536 \quad 43.160 \quad-0.650 \quad 5.990 \quad 0.380 \quad 0.400$ BLUE_GPS 236.740 47.955 5.690 5.160 $0.380 \quad 0.400$ BLYN_GNI 237.072 $48.016 \quad 4.484 \quad 4.835 \quad 0.737 \quad 0.768$ BM31_GPS 238.341 $48.273 \quad 2.670 \quad 1.950 \quad 0.580 \quad 0.590$ BOLI_GPS $236.164 \quad 42.792 \quad-0.020 \quad 9.350 \quad 0.350 \quad 0.350$ BOUN_GPS $237.004 \quad 49.078 \quad 3.680 \quad 3.990 \quad 1.230 \quad 0.940$ BOVL_GPS $243.598 \quad 46.857 \quad 1.160 \quad 0.480 \quad 0.750 \quad 0.700$ BRAE_GPS $236.588 \quad 45.065 \quad 3.670 \quad 6.520 \quad 0.950 \quad 0.970$ BREW_GPS $240.31748 .132 \quad 1.370 \quad 1.600 \quad 0.610 \quad 0.640$ BRIB_GPS 237.847 $37.919 \quad-14.986 \quad 16.112 \quad 0.418 \quad 0.415$ BRMU_GPS 295.304 $32.370 \quad 0.662 \quad-0.271 \quad 0.435 \quad 0.386$ BROW_GPS $237.556 \quad 47.306 \quad 3.730 \quad 2.800 \quad 0.980 \quad 1.180$ BRWV_GPS $234.360 \quad 50.125 \quad 4.390 \quad 4.480 \quad 0.760 \quad 0.790$ BSTR_GPS $236.274 \quad 43.660 \quad 2.230 \quad 8.720 \quad 0.610 \quad 0.620$ $\begin{array}{lllllllll}\text { BULC_GPS } & 232.890 & 50.961 & -1.250 & 4.420 & 0.860 & 0.910\end{array}$ BURN_GPS 242.156 42.780 $-1.901 \quad 1.618 \quad 0.541 \quad 0.541$ BUST_GLA $243.549 \quad 36.745 \quad-3.490 \quad 0.120 \quad 0.380 \quad 0.370$ C033_GPS $240.189 \quad 47.242 \quad 1.720 \quad 1.550 \quad 0.760 \quad 0.740$ C334_GPS $242.431 \quad 46.486 \quad 0.180 \quad 1.630 \quad 0.630 \quad 0.660$ C715_GPS $236.900 \quad 44.633 \quad 4.210 \quad 7.020 \quad 0.880 \quad 1.140$ CALV_GPS $232.046 \quad 51.544 \quad-1.960 \quad 2.390 \quad 0.910 \quad 0.950$ CAM4_GPS 236.222 $43.002 \quad-0.140 \quad 9.310 \quad 0.430 \quad 0.430$ CANN_GPS $236.040 \quad 45.862 \quad 7.610 \quad 8.580 \quad 0.380 \quad 0.400$ CAPS_GPS $237.401 \quad 48.513 \quad 5.180 \quad 3.210 \quad 0.920 \quad 1.080$ $\begin{array}{llllllll}C B L 1 / G P S & 240.451 & 45.344 & -0.170 & 2.110 & 0.530 & 0.570\end{array}$ CC25_GPS $241.41146 .973 \quad 0.620 \quad 1.760 \quad 0.520 \quad 0.520$ CEDC_GPS $236.154 \quad 45.214 \quad 5.190 \quad 8.280 \quad 0.590 \quad 0.630$ $\begin{array}{lllllllll}\text { CHAB_1PS } & 237.881 & 37.724 & -16.608 & 18.972 & 0.357 & 0.414\end{array}$ CHAS_GPS $237.009 \quad 45.524 \quad 3.180 \quad 6.140 \quad 0.420 \quad 0.470$ CHAT_GPS $183.434-43.956-38.713 \quad 50.072 \quad 0.461 \quad 0.480$ CHEM_GPS $236.297 \quad 48.920 \quad 4.863 \quad 4.379 \quad 0.550 \quad 0.555$ $\begin{array}{llllllll}\text { CHLO_GLA } 243.234 & 36.747 & -3.600 & 0.560 & 0.380 & 0.370\end{array}$ CHO1_GPS $238.335 \quad 39.433-10.947 \quad 6.924 \quad 0.509 \quad 0.508$ $\begin{array}{lllllllll}\text { CHUR_GPS } & 265.911 & 58.759 & 0.629 & -0.261 & 0.386 & 0.400\end{array}$ CHWK_GPS $237.992 \quad 49.157 \quad 2.370 \quad 2.150 \quad 0.320 \quad 0.320$ $\begin{array}{llllllll}\text { CHZZ GPS } 236.022 & 45.487 & 6.920 & 9.647 & 0.418 & 0.421\end{array}$ CLAT GPS $236.795 \quad 46.105 \quad 4.730 \quad 6.810 \quad 1.060 \quad 1.110$ CLCC_GPS 237.425 45.326 $1.750 \quad 8.010 \quad 1.040 \quad 1.270$ CLFH_GPS $237.63945 .296 \quad 1.340 \quad 5.100 \quad 0.590 \quad 0.640$ CMB0_GPS $239.614 \quad 38.034 \quad-10.903 \quad 7.929 \quad 0.443 \quad 0.442$ 
$\begin{array}{llllllll}\text { CME1_GPS } & 235.604 & 40.442 & -9.170 & 30.720 & 0.568 & 0.568\end{array}$

$\begin{array}{lllllll}\text { CNDR GPS } 238.722 & 37.896 & -12.961 & 7.586 & 0.485 & 0.482\end{array}$

CNSP_GPS $236.326 \quad 48.465 \quad 5.740 \quad 4.800 \quad 1.310 \quad 1.010$

CNTR_GPS 236.297 $46.973 \quad 8.500 \quad 7.370 \quad 0.520 \quad 0.590$

COEU_GPS $243.204 \quad 47.743 \quad 0.860 \quad 0.160 \quad 0.520 \quad 0.510$

COLR_GPS $237.170 \quad 44.208 \quad 2.350 \quad 6.400 \quad 1.000 \quad 1.020$

COND GPS $236.083 \quad 46.057 \quad 7.800 \quad 7.710 \quad 0.550 \quad 0.580$

CORV_GPS $236.695 \quad 44.586 \quad 3.026 \quad 7.194 \quad 0.361 \quad 0.424$

COVE_GPS 242.180 $45.297 \quad-0.740 \quad 0.820 \quad 0.790 \quad 1.030$

COXI_GPS $231.401 \quad 50.813 \quad-0.190 \quad 2.910 \quad 0.900 \quad 0.950$

CPXF_GNI 237.743 $46.840 \quad 2.540 \quad 4.531 \quad 0.593 \quad 0.611$

CRAT_GLA $243.431 \quad 36.808 \quad-3.390 \quad 0.280 \quad 0.380 \quad 0.370$

CRES_GPS $238.056 \quad 43.530 \quad-0.570 \quad 5.040 \quad 0.900 \quad 0.750$

CROW GPS $236.681 \quad 43.986 \quad 1.920 \quad 8.300 \quad 0.670 \quad 0.710$

CRWN_GPS $241.014 \quad 47.971 \quad 1.360 \quad-0.040 \quad 1.190 \quad 1.100$

CSTL_GPS $237.081 \quad 46.283 \quad 3.750 \quad 6.440 \quad 0.620 \quad 0.690$

CTHS_GPS $241.618 \quad 47.127 \quad 0.550 \quad 1.620 \quad 0.960 \quad 1.150$

CUGR_GPS $237.765 \quad 44.119 \quad 0.980 \quad 5.120 \quad 1.210 \quad 1.030$

CURT_GPS $236.401 \quad 44.377 \quad 3.490 \quad 8.260 \quad 0.500 \quad 0.530$

D639_GPS $240.177 \quad 45.813 \quad-0.040 \quad 2.420 \quad 0.490 \quad 0.540$

D706_GPS $241.068 \quad 43.960 \quad-1.410 \quad 1.270 \quad 0.760 \quad 0.710$

DALL_GPS $238.825 \quad 45.618 \quad 1.390 \quad 3.340 \quad 0.350 \quad 0.410$

DARL_GPS $236.607 \quad 43.242 \quad-1.670 \quad 9.390 \quad 0.930 \quad 0.730$

DAVI_GPS $235.680 \quad 49.599 \quad 4.430 \quad 5.200 \quad 0.540 \quad 0.570$

DDSN_GPS $236.756 \quad 43.119 \quad 0.395 \quad 7.794 \quad 0.520 \quad 0.472$

DELI_GPS $236.545 \quad 43.126 \quad 0.060 \quad 7.920 \quad 0.730 \quad 0.790$

$\begin{array}{llllllll}\mathrm{DIAB} G P S & 238.084 & 37.879 & -12.327 & 11.091 & 0.458 & 0.456\end{array}$

DISC GPS $236.773 \quad 48.425 \quad 4.610 \quad 4.660 \quad 1.370 \quad 0.990$

DOTT_GPS $236.725 \quad 46.631 \quad 4.850 \quad 6.810 \quad 0.460 \quad 0.520$

DOUG_GPS $236.653 \quad 48.493 \quad 4.970 \quad 4.020 \quad 1.300 \quad 0.930$

DUBO_GPS $264.134 \quad 50.259 \quad-0.640 \quad-0.762 \quad 0.367 \quad 0.332$

DUNG_GPS $236.890 \quad 48.181 \quad 6.730 \quad 3.170 \quad 1.200 \quad 1.230$

DUWA_GPS $237.613 \quad 47.595 \quad 4.870 \quad 5.340 \quad 1.170 \quad 1.460$

E040_GPS $239.820 \quad 45.243 \quad 1.320 \quad 1.930 \quad 0.760 \quad 0.760$

E072_GPS $243.588 \quad 43.075 \quad-1.960 \quad 0.420 \quad 0.970 \quad 1.000$

E141_GPS 236.709 44.391 $4.720 \quad 7.210 \quad 0.590 \quad 0.630$

E518_GPS 239.408 $47.559 \quad 3.290 \quad 2.680 \quad 1.250 \quad 0.950$

EA40_GPS $236.591 \quad 47.939 \quad 6.810 \quad 4.310 \quad 0.450 \quad 0.470$

EARL_GPS $236.024 \quad 49.753 \quad 3.520 \quad 4.250 \quad 0.480 \quad 0.520$

EASN_GPS $238.829 \quad 47.232 \quad 4.950 \quad 4.160 \quad 0.470 \quad 0.480$

$\begin{array}{lllllll}\text { ECHO_GPS } 245.736 & 37.916 & -3.442 & -0.423 & 0.367 & 0.364\end{array}$

EDDY_GPS $236.233 \quad 44.612 \quad 5.420 \quad 7.950 \quad 1.040 \quad 1.250$

EGAN_GPS $245.06139 .345-4.922-0.1960 .4360 .436$

ELIZ_GPS $232.877 \quad 49.873 \quad 5.180 \quad 6.260 \quad 0.700 \quad 0.730$

$\begin{array}{llllllll}\text { ELKO_GPS } 244.183 & 40.915 & -4.255 & 0.130 & 0.400 & 0.364\end{array}$

ELKR_GPS $237.655 \quad 46.301 \quad 3.715 \quad 4.539 \quad 0.568 \quad 0.574$

EOUT_GPS $248.071 \quad 41.253-1.362-0.151 \quad 0.427 \quad 0.361$

ESTA_GPS $237.667 \quad 45.299 \quad 1.350 \quad 5.100 \quad 0.590 \quad 0.640$

EZEL_GPS $236.837 \quad 44.079 \quad 1.990 \quad 7.120 \quad 0.820 \quad 0.940$

F408_GPS 243.551 $44.669 \quad 0.850 \quad 1.230 \quad 0.890 \quad 0.640$

F735_GPS $238.803 \quad 44.269-0.6204 .170 \quad 1.310 \quad 1.270$

F751_GPS 235.801 $43.340 \quad 4.490 \quad 11.560 \quad 0.970 \quad 1.110$

F760_GPS $237.375 \quad 42.142 \quad-3.230 \quad 5.430 \quad 0.770 \quad 0.890$

FAAS_GPS $243.881 \quad 45.942 \quad-0.250 \quad-0.020 \quad 0.740 \quad 0.840$

FALL_GPS $238.867 \quad 46.629 \quad 2.580 \quad 2.700 \quad 0.810 \quad 0.750$

FARB_GPS $236.999 \quad 37.697 \quad-28.543 \quad 37.791 \quad 0.573 \quad 0.571$

FARO_GPS $236.661 \quad 42.423-1.860 \quad 8.540 \quad 0.600 \quad 0.660$

FARV_GPS $237.347 \quad 43.586 \quad-0.050 \quad 7.450 \quad 0.660 \quad 0.750$

FARW_GPS $236.624 \quad 47.015 \quad 5.600 \quad 6.690 \quad 0.500 \quad 0.560$

FERR_GPS $241.845 \quad 48.278 \quad-0.600 \quad 0.010 \quad 1.290 \quad 1.710$ 
$\begin{array}{lllllll}\text { FHAM_GPS } & 236.140 & 44.019 & 4.520 & 9.560 & 1.560 & 1.540\end{array}$ FILB GPS $234.302 \quad 49.883 \quad 4.430 \quad 4.210 \quad 0.750 \quad 0.790$ FISH_GPS 235.732 $42.046 \quad 2.692 \quad 10.6390 .622 \quad 0.570$ FLAT_GPS $244.685 \quad 46.339 \quad 0.890 \quad 0.480 \quad 0.690 \quad 0.760$ FLIN_GPS $258.02254 .726-0.121-1.188 \quad 0.328 \quad 0.342$ FORE_GPS $241.042 \quad 45.138 \quad 0.500 \quad 1.260 \quad 0.690 \quad 0.830$ FORK_GPS 235.604 47.937 $12.920 \quad 9.180 \quad 0.530 \quad 0.540$ FOSS_GPS $239.780 \quad 45.000 \quad-0.750 \quad 2.150 \quad 0.920 \quad 0.920$ FOUR_GPS $234.718 \quad 49.192 \quad 6.410 \quad 6.160 \quad 0.600 \quad 0.630$ FRAN_GPS $236.500 \quad 46.541 \quad 6.110 \quad 7.650 \quad 0.480 \quad 0.510$ FRDC_GPS $234.493 \quad 48.985 \quad 9.080 \quad 9.010 \quad 0.790 \quad 0.890$ $\begin{array}{lllllll}\text { FRED_GPS } & 247.501 & 36.988 & -1.830 & -0.240 & 0.384 & 0.412\end{array}$ FRND_GPS $237.397 \quad 48.892 \quad 3.580 \quad 3.540 \quad 1.060 \quad 1.350$ FTS1_GPS $236.044 \quad 46.205 \quad 8.520 \quad 8.740 \quad 0.260 \quad 0.260$ G118_GPS $240.048 \quad 42.098 \quad-3.520 \quad 3.680 \quad 0.670 \quad 0.750$ G370_GPS $238.731 \quad 48.674 \quad 2.810 \quad 2.340 \quad 0.610 \quad 0.620$ G404_GPS $243.627 \quad 46.142 \quad 0.380 \quad 0.430 \quad 0.720 \quad 0.830$ G753_GPS 239.491 $44.353-0.950 \quad 1.840 \quad 1.370 \quad 1.120$ GABR_GPS $236.180 \quad 49.203 \quad 4.570 \quad 4.390 \quad 0.700 \quad 0.660$ GARL_GPS $240.645 \quad 40.417 \quad-5.592 \quad 2.830 \quad 0.391 \quad 0.354$ GARY_GPS $236.930 \quad 45.612 \quad 2.970 \quad 5.650 \quad 0.460 \quad 0.480$ GENT_GPS $241.923 \quad 43.744 \quad-1.810 \quad 1.120 \quad 1.200 \quad 0.840$ GLAC_GPS $234.636 \quad 49.552 \quad 5.640 \quad 4.300 \quad 0.710 \quad 0.760$ GLDH_GPS $237.213 \quad 47.549 \quad 5.420 \quad 4.590 \quad 0.410 \quad 0.430$ G089_GPS 235.572 $42.421 \quad 3.710 \quad 12.820 \quad 0.440 \quad 0.390$ GOBS_GPS $239.185 \quad 45.839 \quad 1.026 \quad 3.277 \quad 0.543 \quad 0.543$ GODE GPS $283.173 \quad 39.022 \quad 0.267 \quad-0.008 \quad 0.298 \quad 0.297$ GP14_GPS $235.822 \quad 47.114 \quad 13.540 \quad 12.580 \quad 0.450 \quad 0.500$ GP17_GPS $238.375 \quad 47.138 \quad 2.490 \quad 2.850 \quad 0.630 \quad 0.640$ GP29_GPS 238.406 $48.486 \quad 1.160 \quad 2.290 \quad 0.580 \quad 0.570$ GP35_GPS $236.351 \quad 46.334 \quad 6.730 \quad 7.080 \quad 0.610 \quad 0.640$ GP37_GPS $237.857 \quad 48.917 \quad 2.560 \quad 1.700 \quad 0.700 \quad 0.860$ GRAY_GPS $235.900 \quad 46.903 \quad 10.840 \quad 10.940 \quad 0.470 \quad 0.480$ GREN_GPS $237.474 \quad 41.555 \quad-4.430 \quad 4.380 \quad 1.130 \quad 1.390$ GREV_GPS $235.721 \quad 47.304 \quad 14.790 \quad 12.390 \quad 0.330 \quad 0.350$ GREY_GPS 235.296 $48.996 \quad 7.240 \quad 7.160 \quad 0.630 \quad 0.670$ GRND_GPS $236.361 \quad 45.082 \quad 4.210 \quad 6.490 \quad 0.470 \quad 0.480$ GRSM_GPS $238.223 \quad 48.539 \quad 3.400 \quad 3.870 \quad 0.830 \quad 1.030$ GTRG_GPS $246.759 \quad 43.244 \quad-2.278-0.261 \quad 0.430 \quad 0.367$ GWEN_GPS $238.672 \quad 45.783 \quad 2.290 \quad 2.310 \quad 0.970 \quad 0.970$ H318_GPS $243.813 \quad 41.962 \quad-3.210 \quad 0.600 \quad 0.760 \quad 0.710$ H428_GPS 239.785 $45.745 \quad 0.760 \quad 2.130 \quad 1.300 \quad 1.510$ HAFF_GPS $237.824 \quad 47.485 \quad 3.260 \quad 3.900 \quad 0.700 \quad 0.740$ HAMI_GPS $235.935 \quad 44.602 \quad 6.590 \quad 8.960 \quad 0.420 \quad 0.440$ HAND_GPS $235.041 \quad 49.069 \quad 6.120 \quad 6.110 \quad 0.600 \quad 0.630$ HANS_GPS $236.430 \quad 45.885 \quad 5.100 \quad 6.580 \quad 0.500 \quad 0.520$ HARD_GPS 232.619 50.698 $-0.110 \quad 3.310 \quad 0.770 \quad 0.800$ HEAD_GPS $235.924 \quad 46.300 \quad 9.560 \quad 10.480 \quad 0.730 \quad 0.740$ HEBE_GPS $248.627 \quad 40.514 \quad-0.934-0.2390 .4090 .408$ HELE_GPS $237.199 \quad 45.857 \quad 2.980 \quad 6.940 \quad 0.400 \quad 0.440$ HKUS_GPS $234.159 \quad 50.335 \quad 3.370 \quad 4.180 \quad 0.760 \quad 0.780$ HLID_GPS $245.586 \quad 43.563-2.135-0.137 \quad 0.384 \quad 0.444$ HOLB_GPS $231.865 \quad 50.640 \quad 0.110 \quad 3.730 \quad 0.630 \quad 0.630$ HOPB_GPS $236.925 \quad 38.995 \quad-18.965 \quad 21.068 \quad 0.427 \quad 0.424$ HOWE_GPS $237.120 \quad 44.990 \quad 2.810 \quad 6.810 \quad 0.470 \quad 0.490$ HRMA_GPS $240.741 \quad 45.827 \quad-0.250 \quad 1.380 \quad 0.490 \quad 0.510$ HUBB GPS $237.189 \quad 45.179 \quad 4.150 \quad 7.270 \quad 1.070 \quad 1.280$ HUR2_GPS $236.471 \quad 47.990 \quad 6.600 \quad 5.650 \quad 0.450 \quad 0.470$ HUSB_GPS $238.151 \quad 44.120 \quad-3.350 \quad 11.080 \quad 0.770 \quad 0.790$ HYAK_GPS $238.608 \quad 47.388 \quad 2.930 \quad 2.350 \quad 1.070 \quad 1.330$ 
ICEB GPS $237.115 \quad 48.420 \quad 4.950 \quad 4.140 \quad 0.580 \quad 0.500$

ICEH_GPS $241.120 \quad 46.251 \quad-0.780 \quad 1.480 \quad 1.420 \quad 1.180$

ILLA_GPS $236.318 \quad 42.104 \quad-1.157 \quad 8.633 \quad 0.791 \quad 0.682$

IMP0_GPS $237.174 \quad 44.795 \quad 2.840 \quad 6.320 \quad 0.540 \quad 0.580$

ISLE_GPS $236.051 \quad 45.273 \quad 5.680 \quad 9.480 \quad 1.410 \quad 1.530$

J090_GPS $240.143 \quad 44.119 \quad-0.810 \quad 2.270 \quad 0.480 \quad 0.510$

JAIL_GPS $237.029 \quad 44.876 \quad 2.610 \quad 6.390 \quad 0.980 \quad 1.140$

JENS_GPS $231.73550 .646-1.470 \quad 3.840 \quad 0.920 \quad 0.940$

JHRT_GPS $234.603 \quad 50.033 \quad 4.160 \quad 4.330 \quad 0.760 \quad 0.780$

JOHN_GLA $243.901 \quad 36.459 \quad-3.320 \quad-0.260 \quad 0.380 \quad 0.370$

JONY_GPS $240.372 \quad 44.623 \quad-0.800 \quad 1.140 \quad 0.670 \quad 0.670$

JOSE_GPS $242.748 \quad 45.349 \quad-0.370 \quad 0.390 \quad 0.540 \quad 0.570$

JRDR_GPS $235.897 \quad 48.465 \quad 6.730 \quad 5.240 \quad 1.260 \quad 1.080$

$\begin{array}{llllllll}\text { JUNC GPS } & 239.053 & 44.920 & 0.360 & 2.598 & 0.532 & 0.550\end{array}$

JUST_GPS $237.123 \quad 42.323 \quad-2.460 \quad 7.360 \quad 0.460 \quad 0.530$

JUSW_GPS $242.941 \quad 48.179 \quad 0.870 \quad 0.050 \quad 0.600 \quad 0.670$

K024_GPS 242.268 $48.208 \quad 0.430 \quad 1.410 \quad 0.870 \quad 1.000$

KAMI_GPS $243.995 \quad 46.213 \quad 1.280 \quad 1.260 \quad 0.610 \quad 0.680$

KELL_GPS $241.341 \quad 48.698 \quad 1.080 \quad 0.970 \quad 0.930 \quad 0.650$

KELS_GPS $237.104 \quad 46.118 \quad 3.700 \quad 6.145 \quad 0.444 \quad 0.447$

KING_GPS $232.22951 .854 \quad-3.740 \quad 2.470 \quad 0.930 \quad 0.960$

KINW_GPS $237.352 \quad 47.735 \quad 5.730 \quad 4.620 \quad 0.670 \quad 0.810$

KLAS_GPS $236.280 \quad 46.089 \quad 6.480 \quad 7.210 \quad 0.640 \quad 0.710$

KLUC_GPS $232.836 \quad 50.573 \quad 1.360 \quad 4.700 \quad 1.200 \quad 1.230$

KOPR_GPS 232.101 50.486 2.750 $2.860 \quad 0.840 \quad 0.860$

KTBW_GNI $237.205 \quad 47.547 \quad 3.851 \quad 4.259 \quad 0.442 \quad 0.442$

KWJ1 GPS $167.730 \quad 8.722 \quad-69.881 \quad 44.642 \quad 0.559 \quad 0.534$

L387_GPS $239.937 \quad 47.656 \quad 1.300 \quad 1.680 \quad 0.600 \quad 0.570$

LANG_GPS $237.044 \quad 44.566 \quad 2.790 \quad 6.300 \quad 0.600 \quad 0.620$

LARC_GPS $237.912 \quad 45.533 \quad 1.630 \quad 7.640 \quad 1.180 \quad 1.210$

LATA_GPS $242.868 \quad 47.274 \quad 1.050 \quad 1.300 \quad 0.660 \quad 0.770$

LAZA_GPS $236.176 \quad 48.612 \quad 5.170 \quad 4.170 \quad 1.150 \quad 0.980$

LESL_GPS $236.762 \quad 43.706 \quad 1.500 \quad 6.380 \quad 0.680 \quad 0.830$

LEV1_GPS $226.907 \quad 56.466 \quad-3.165 \quad 2.754 \quad 0.602 \quad 0.682$

LEWG_GPS $237.379 \quad 47.094 \quad 3.670 \quad 6.540 \quad 0.570 \quad 0.630$

LINH_GPS $239.461 \quad 47.000 \quad 1.550 \quad 2.210 \quad 0.370 \quad 0.380$

LITT_GLA $243.692 \quad 36.746-3.330-0.190 \quad 0.380 \quad 0.370$

LK42_GPS $236.330 \quad 48.150 \quad 6.260 \quad 5.130 \quad 0.780 \quad 0.850$

LKCP_GNI $238.169 \quad 47.944 \quad 2.026 \quad 3.293 \quad 0.492 \quad 0.472$

$\begin{array}{llllllll} & \text { LKWY GPS } & 249.600 & 44.565 & 0.221 & -3.584 & 0.364 & 0.574\end{array}$

LMUT_GPS $248.072 \quad 40.261-2.821-0.066 \quad 0.719 \quad 0.735$

LOPE_GPS $240.646 \quad 41.997 \quad-3.740 \quad 2.470 \quad 0.660 \quad 0.700$

LOWL_GPS $237.205 \quad 43.922 \quad 2.200 \quad 5.630 \quad 1.080 \quad 1.070$

LSII_GPS $240.663 \quad 47.185 \quad 0.580 \quad 0.950 \quad 0.450 \quad 0.480$

LUCA_GPS $235.941 \quad 42.552 \quad 2.160 \quad 10.730 \quad 1.320 \quad 1.200$

LUTZ_GPS $238.135 \quad 37.287 \quad-20.350 \quad 22.834 \quad 0.456 \quad 0.453$

M746_GPS $235.678 \quad 42.183 \quad 3.150 \quad 13.720 \quad 0.760 \quad 0.700$

MACK_GPS $236.869 \quad 45.190 \quad 2.910 \quad 7.370 \quad 1.070 \quad 1.140$

MAWY_GPS $249.311 \quad 44.973 \quad 0.563 \quad 0.599 \quad 0.397 \quad 0.424$

MDMT_GPS 238.778 $42.418 \quad-5.226 \quad 6.176 \quad 0.602 \quad 0.587$

MENZ_GPS $234.503 \quad 50.231 \quad 2.580 \quad 4.440 \quad 0.740 \quad 0.750$

MERC_GLA $244.021 \quad 36.633 \quad-3.240 \quad-0.550 \quad 0.380 \quad 0.370$

MESA GPS $240.993 \quad 46.621 \quad 0.310 \quad 1.500 \quad 0.560 \quad 0.520$

MHCB_GPS $238.357 \quad 37.342 \quad-14.120 \quad 10.815 \quad 0.370 \quad 0.368$

MILL_GPS $237.520 \quad 44.752 \quad 2.250 \quad 5.880 \quad 0.530 \quad 0.580$

MKEA_GPS 204.544 19.801 $\quad-60.500 \quad 51.831 \quad 0.386 \quad 0.384$

MOAK_GPS 233.942 $50.104 \quad 4.630 \quad 4.060 \quad 0.780 \quad 0.820$

MODB_1PS $239.697 \quad 41.902 \quad-4.750 \quad 3.660 \quad 0.510 \quad 0.510$

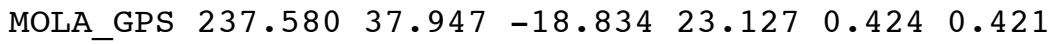

MONB_GPS $238.133 \quad 37.485 \quad-16.218 \quad 16.149 \quad 0.458 \quad 0.456$ 
MOON_GPS $236.16544 .777 \quad 5.220 \quad 8.370 \quad 0.500 \quad 0.460$ MORT GPS $237.730 \quad 46.551 \quad 3.440 \quad 5.120 \quad 0.320 \quad 0.330$ MRS1_GNI $238.065 \quad 47.142 \quad 2.320 \quad 3.890 \quad 0.460 \quad 0.480$ MRY5_GPS 236.448 $44.504 \quad 4.150 \quad 7.850 \quad 0.570 \quad 0.560$ MTAD_GPS $238.458 \quad 46.000 \quad 2.140 \quad 5.010 \quad 0.830 \quad 0.860$ N274_GPS $236.212 \quad 45.907 \quad 5.420 \quad 8.350 \quad 0.680 \quad 0.730$ N469GPS $235.881 \quad 48.198 \quad 8.340 \quad 6.800 \quad 0.630 \quad 0.780$ N748_GPS $236.618 \quad 42.621 \quad-2.560 \quad 8.710 \quad 1.540 \quad 1.420$ NACH_GPS $235.001 \quad 49.949 \quad 4.540 \quad 5.020 \quad 0.720 \quad 0.750$ NAIU_GPS $247.770 \quad 41.016-1.540 \quad-0.860 \quad 0.320 \quad 0.310$ NANO_GPS $235.914 \quad 49.295 \quad 4.480 \quad 4.920 \quad 0.330 \quad 0.320$ NBCG_GPS $235.401 \quad 48.370 \quad 9.670 \quad 8.210 \quad 0.500 \quad 0.500$ NEAH_GPS 235.375 $48.298 \quad 10.238 \quad 8.419 \quad 0.466 \quad 0.492$ NESK_GPS $236.034 \quad 45.134 \quad 7.360 \quad 9.320 \quad 0.390 \quad 0.400$ NESM GPS $236.772 \quad 44.925 \quad 3.410 \quad 7.410 \quad 0.620 \quad 0.650$ NOMT_GPS $248.370 \quad 45.597 \quad 0.123 \quad-0.510 \quad 0.430 \quad 0.433$ NTKA_GPS $233.383 \quad 49.592 \quad 6.690 \quad 8.500 \quad 0.700 \quad 0.730$ OFWY_GPS $249.168 \quad 44.452 \quad-3.512-0.534 \quad 0.826 \quad 0.763$ OGIL_GPS $241.039 \quad 44.410 \quad-1.660 \quad 1.910 \quad 0.740 \quad 0.710$ OHLN_GPS $237.727 \quad 38.006 \quad-15.935 \quad 17.510 \quad 0.778 \quad 0.778$ OHME_GNI $239.674 \quad 47.480 \quad 2.650 \quad 1.640 \quad 0.970 \quad 1.000$ OKAY_GPS $235.736 \quad 49.228 \quad 4.910 \quad 5.200 \quad 0.510 \quad 0.530$ OLYM_GPS $237.092 \quad 46.967 \quad 3.990 \quad 5.600 \quad 0.380 \quad 0.320$ OP25_GPS $236.076 \quad 46.972 \quad 10.380 \quad 8.700 \quad 0.450 \quad 0.470$ ORCC_GPS $237.339 \quad 47.446 \quad 4.730 \quad 3.620 \quad 0.990 \quad 1.150$ ORVB_GPS $238.500 \quad 39.555 \quad-10.737 \quad 6.620 \quad 0.427 \quad 0.424$ OVER GPS $237.858 \quad 47.636 \quad 2.700 \quad 3.760 \quad 0.870 \quad 0.990$ OYST_GPS $234.600 \quad 49.823 \quad 4.690 \quad 4.910 \quad 0.730 \quad 0.770$ P75Z_GPS 235.825 43.124 $1.990 \quad 10.090 \quad 0.540 \quad 0.560$ PABH_GPS 235.795 $47.213 \quad 13.566 \quad 11.745 \quad 0.381 \quad 0.381$ PACH_GPS $234.957 \quad 48.865 \quad 8.180 \quad 7.170 \quad 0.560 \quad 0.580$ PACI_GPS $237.125 \quad 42.336 \quad-1.520 \quad 6.820 \quad 1.610 \quad 0.860$ PACK_GPS $238.325 \quad 46.607 \quad 2.000 \quad 4.530 \quad 0.970 \quad 0.990$ PAIN_GPS $237.728 \quad 47.908 \quad 2.920 \quad 3.040 \quad 0.810 \quad 0.880$ $\begin{array}{llllllll}\text { PAIS_GPS } & 239.451 & 42.704 & -2.220 & 2.590 & 0.780 & 0.920\end{array}$ PARK_GPS 236.540 $46.268 \quad 6.650 \quad 8.130 \quad 0.500 \quad 0.570$ PAUL_GPS $240.969 \quad 45.499 \quad 0.670 \quad 1.690 \quad 0.870 \quad 0.870$ PBL1_GPS $237.581 \quad 37.853 \quad-23.104 \quad 21.666 \quad 0.433 \quad 0.430$ PETE_GPS 237.031 $44.510 \quad 2.410 \quad 6.580 \quad 0.740 \quad 0.820$ PGC4_GPS $236.549 \quad 48.648 \quad 4.700 \quad 4.120 \quad 0.530 \quad 0.530$ PIER_GPS 233.878 $49.619 \quad 7.000 \quad 5.230 \quad 0.730 \quad 0.770$ PLUD_GPS $237.317 \quad 47.922 \quad 5.020 \quad 3.780 \quad 0.620 \quad 0.700$ POCA_GPS $235.555 \quad 49.710 \quad 3.570 \quad 4.560 \quad 0.580 \quad 0.640$ POIN_GLA $243.880 \quad 36.580 \quad-3.170 \quad-0.320 \quad 0.380 \quad 0.370$ PONS_GPS $235.88344 .170 \quad 5.910 \quad 9.020 \quad 1.490 \quad 1.500$ POTB_GPS 238.065 38.203 -11.287 $8.540 \quad 0.461 \quad 0.458$ POWE_GPS 235.544 $49.807 \quad 4.560 \quad 4.370 \quad 0.600 \quad 0.670$ PPT1_GPS $237.610 \quad 37.187 \quad-28.966 \quad 35.352 \quad 0.433 \quad 0.430$ PRAI_GPS $236.176 \quad 45.421 \quad 4.940 \quad 9.060 \quad 1.090 \quad 1.290$ PRDS_GPS $245.707 \quad 50.871 \quad 0.653-1.280 \quad 0.458 \quad 0.456$ PRES_GPS $237.075 \quad 46.040 \quad 3.300 \quad 7.080 \quad 0.750 \quad 0.650$ PRIN_GPS 239.135 $44.301 \quad-0.430 \quad 4.190 \quad 0.580 \quad 0.550$ PROS_GPS 237.489 $42.741 \quad-1.890 \quad 6.770 \quad 0.710 \quad 0.800$ PTAL_GPS 235.139 49.256 5.300 5.160 $0.730 \quad 0.720$ PTAN_GPS $236.505 \quad 48.117 \quad 5.190 \quad 5.270 \quad 0.660 \quad 0.650$ PTHY_GPS 232.625 50.686 $\quad-0.100 \quad 4.850 \quad 0.730 \quad 0.700$ PTS5_GPS $237.280 \quad 46.533 \quad 4.530 \quad 5.600 \quad 0.560 \quad 0.660$ PTSG_GPS $235.745 \quad 41.783 \quad 2.599 \quad 11.685 \quad 0.392 \quad 0.373$ PUPU_GNI $237.992 \quad 47.500 \quad 2.698 \quad 2.735 \quad 0.500 \quad 0.502$ QUIN_GPS 239.056 39.975 -9.377 6.451 $0.418 \quad 0.412$ 
R378_GPS $239.744 \quad 48.508 \quad-0.380 \quad 3.770 \quad 0.500 \quad 0.530$

R409-GPS $242.751 \quad 45.929 \quad 0.410 \quad 0.500 \quad 0.460 \quad 0.490$

R489_GPS $242.543 \quad 44.578 \quad-1.250 \quad 1.380 \quad 0.720 \quad 0.590$

RADA_GPS $234.159 \quad 49.084 \quad 9.310 \quad 7.380 \quad 0.600 \quad 0.650$

RBUT_GPS $248.191 \quad 40.781 \quad-1.360 \quad-0.110 \quad 0.330 \quad 0.320$

REDM_GPS $238.852 \quad 44.260 \quad-0.013 \quad 3.521 \quad 0.515 \quad 0.515$

REPO_GLA $243.532 \quad 36.840 \quad-3.256 \quad-1.988 \quad 1.014 \quad 1.016$

REST_GPS $236.539 \quad 45.797 \quad 4.620 \quad 7.430 \quad 0.480 \quad 0.480$

REUB_GPS $236.403 \quad 42.719 \quad-1.220 \quad 8.900 \quad 0.340 \quad 0.340$

RKBU_GPS $237.434 \quad 45.547 \quad 2.340 \quad 5.970 \quad 0.340 \quad 0.360$

RKPT_GPS $237.900 \quad 42.473 \quad-3.290 \quad 6.300 \quad 0.700 \quad 0.810$

$\begin{array}{lllllll}\text { ROBI_GPS } & 232.399 & 51.186 & -1.830 & 2.700 & 0.860 & 0.890\end{array}$

ROG2_GPS $235.572 \quad 42.430 \quad 4.200 \quad 13.170 \quad 0.970 \quad 0.900$

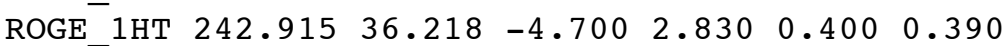

ROSE_GPS $236.645 \quad 43.246 \quad-0.890 \quad 7.520 \quad 0.400 \quad 0.410$

ROSW_GPS $240.263 \quad 48.394 \quad 0.230 \quad 1.540 \quad 0.870 \quad 0.840$

RPT1_GPS $237.625 \quad 47.388 \quad 3.230 \quad 4.000 \quad 0.390 \quad 0.390$

$\begin{array}{llllllll}\text { RYAN_1HT } & 243.350 & 36.316 & -3.591 & 1.061 & 0.594 & 0.594\end{array}$

S262 GPS $242.950 \quad 46.426 \quad 2.010 \quad 0.130 \quad 0.720 \quad 0.660$

S300_GPS $238.442 \quad 37.667 \quad-11.777 \quad 8.919 \quad 0.437 \quad 0.437$

S381_GPS $243.742 \quad 48.731 \quad 1.260 \quad-0.130 \quad 0.580 \quad 0.650$

S389_GPS $239.930 \quad 48.157 \quad 0.920 \quad 1.450 \quad 0.510 \quad 0.550$

S418_GPS $244.162 \quad 42.880 \quad-2.760 \quad-0.410 \quad 0.810 \quad 0.840$

S509_GPS $241.287 \quad 47.761 \quad 1.380 \quad 0.680 \quad 1.120 \quad 0.850$

SARD_GPS $237.686 \quad 43.963 \quad 1.770 \quad 4.590 \quad 0.650 \quad 0.670$

SARG_GPS $239.523 \quad 46.602 \quad 0.970 \quad 1.820 \quad 0.570 \quad 0.610$

$\begin{array}{lllllll}\text { SATS GPS } & 236.459 & 46.966 & 6.010 & 7.530 & 0.760 & 0.810\end{array}$

SATU GPS $236.829 \quad 48.774 \quad 4.220 \quad 4.190 \quad 1.330 \quad 1.050$

SC00_GNI 239.275 $46.951 \quad 1.442 \quad 2.191 \quad 0.502 \quad 0.485$

SC02_GNI $236.992 \quad 48.546 \quad 3.529 \quad 3.397 \quad 0.608 \quad 0.594$

SCAR_GPS $231.992 \quad 50.654 \quad 1.090 \quad 2.650 \quad 0.860 \quad 0.880$

SCAZ_GPS $236.796 \quad 45.469 \quad 4.660 \quad 6.050 \quad 1.330 \quad 1.450$

SCHO_GPS $237.125 \quad 47.824 \quad 6.180 \quad 5.260 \quad 0.790 \quad 0.830$

SCOT_GPS $236.935 \quad 43.372 \quad-0.210 \quad 7.060 \quad 0.350 \quad 0.360$

SDNY_GPS $236.897 \quad 44.780 \quad 3.780 \quad 8.150 \quad 0.830 \quad 0.860$

SDRO_GPS $237.772 \quad 48.508 \quad 3.060 \quad 3.320 \quad 0.520 \quad 0.560$

SEAT_GPS $237.691 \quad 47.654 \quad 3.784 \quad 3.8390 .495 \quad 0.439$

SECH_GPS $236.123 \quad 49.598 \quad 3.590 \quad 4.730 \quad 0.570 \quad 0.630$

$\begin{array}{llllllll}\text { SEDR_GPS } & 237.776 & 48.522 & 2.901 & 3.252 & 0.480 & 0.464\end{array}$

SENT_GPS $234.042 \quad 49.956 \quad 4.550 \quad 3.930 \quad 1.020 \quad 1.040$

$\begin{array}{lllllll}\text { SEYM_GPS } & 232.720 & 51.465 & -0.270 & 3.430 & 0.880 & 0.920\end{array}$

SHEP_GPS $235.813 \quad 49.535 \quad 4.230 \quad 4.740 \quad 0.530 \quad 0.560$

SHER_GPS $236.079 \quad 48.377 \quad 6.900 \quad 4.510 \quad 1.520 \quad 1.230$

SHUS_GPS $232.191 \quad 50.781 \quad-0.670 \quad 3.380 \quad 1.070 \quad 1.090$

SILV_GPS $238.939 \quad 43.125 \quad-2.120 \quad 4.470 \quad 1.450 \quad 1.320$

SISK_GPS $236.303 \quad 45.483 \quad 5.950 \quad 8.570 \quad 0.480 \quad 0.450$

SIST_GPS $238.444 \quad 44.306 \quad 0.924 \quad 3.862 \quad 0.520 \quad 0.527$

SITA_GPS $237.447 \quad 47.571 \quad 1.780 \quad 4.020 \quad 1.590 \quad 1.870$

SKIL_GPS $238.103 \quad 42.318 \quad-3.680 \quad 6.210 \quad 0.880 \quad 0.920$

$\begin{array}{lllllll}\text { SKUL_GLA } & 243.789 & 36.730 & -3.470 & -0.200 & 0.380 & 0.370\end{array}$

SKYO_GPS $238.872 \quad 44.634 \quad 0.050 \quad 1.950 \quad 0.660 \quad 0.830$

SLID_GPS $240.116 \quad 39.314 \quad-9.908 \quad 5.598 \quad 0.486 \quad 0.453$

SLVR_GPS $238.428 \quad 48.079 \quad 2.900 \quad 3.720 \quad 1.200 \quad 1.540$

SMEL_GPS $247.155 \quad 39.426 \quad-3.077 \quad-0.366 \quad 0.400 \quad 0.400$

SMLT_GPS $243.824 \quad 47.546 \quad 0.710 \quad 0.090 \quad 0.580 \quad 0.640$

$\begin{array}{lllllll}\text { SMYC_GLA } & 244.413 & 36.320 & -3.520 & -0.510 & 0.380 & 0.370\end{array}$

SNDR_GPS $236.859 \quad 47.237 \quad 4.550 \quad 6.000 \quad 0.570 \quad 0.580$

SNI1_GPS $240.476 \quad 33.248 \quad-31.784 \quad 34.473 \quad 0.416 \quad 0.404$

SOAM_GPS $236.450 \quad 44.038 \quad 2.730 \quad 7.710 \quad 0.520 \quad 0.560$

SOBE_GPS $236.190 \quad 46.663 \quad 7.630 \quad 9.330 \quad 0.490 \quad 0.550$ 
$\begin{array}{llllllll}\text { SODB_GPS } & 238.074 & 37.166 & -21.958 & 25.524 & 0.477 & 0.476\end{array}$

SOOS GPS $235.886 \quad 43.885 \quad 5.800 \quad 10.740 \quad 0.680 \quad 0.730$

SOR4_GPS $237.521 \quad 42.065 \quad-3.720 \quad 6.340 \quad 0.380 \quad 0.380$

SPAT_GPS $231.673 \quad 50.678 \quad-0.380 \quad 4.820 \quad 0.870 \quad 0.900$

SPIL_GPS $238.700 \quad 46.873 \quad 2.080 \quad 3.080 \quad 0.550 \quad 0.620$

SPN1_GPS $242.576 \quad 47.518 \quad 0.290 \quad 0.340 \quad 0.470 \quad 0.470$

SPOO_GPS $237.836 \quad 47.401 \quad 4.040 \quad 4.830 \quad 0.810 \quad 0.830$

SPRO_GPS $237.126 \quad 45.269 \quad 2.970 \quad 6.250 \quad 0.450 \quad 0.430$

STEV_GPS $238.11745 .692 \quad 2.060 \quad 4.340 \quad 0.610 \quad 0.670$

STJO_GPS $307.322 \quad 47.595 \quad 0.473 \quad 0.039 \quad 0.414 \quad 0.414$

STNN_GPS $240.215 \quad 41.583 \quad-4.020 \quad 2.530 \quad 0.660 \quad 0.690$

STOL_GPS $238.330 \quad 45.304 \quad 1.860 \quad 4.950 \quad 0.500 \quad 0.590$

STRA GPS $234.417 \quad 49.995 \quad 3.300 \quad 4.340 \quad 0.750 \quad 0.790$

STRI GLA $243.662 \quad 36.645 \quad-3.230 \quad 0.150 \quad 0.380 \quad 0.370$

SUAA GPS $237.827 \quad 37.427 \quad-22.133 \quad 25.520 \quad 0.453 \quad 0.450$

T739_GPS $236.681 \quad 43.465 \quad 0.840 \quad 7.750 \quad 1.020 \quad 0.870$

T758_GPS 235.607 $42.586 \quad-1.060 \quad 12.190 \quad 0.980 \quad 0.900$

TAHU_GPS $236.887 \quad 47.387 \quad 5.670 \quad 5.600 \quad 0.450 \quad 0.480$

THR3_GPS $244.897 \quad 42.087 \quad-3.310 \quad 0.910 \quad 0.900 \quad 0.890$

THUN_GPS $237.710 \quad 47.103 \quad 3.940 \quad 4.800 \quad 0.560 \quad 0.580$

TIBB_GPS $237.552 \quad 37.891 \quad-20.026 \quad 24.386 \quad 0.456 \quad 0.453$

TID3_GPS $235.670 \quad 43.350 \quad 5.080 \quad 11.270 \quad 0.580 \quad 0.450$

TIGR_GPS $238.015 \quad 47.509 \quad 2.600 \quad 3.270 \quad 0.400 \quad 0.410$

TIMB_GPS $238.288 \quad 45.334 \quad 2.100 \quad 3.510 \quad 0.630 \quad 0.770$

TOBY_GPS $235.336 \quad 49.490 \quad 4.090 \quad 5.300 \quad 0.670 \quad 0.700$

TOKE_GPS $237.572 \quad 43.228 \quad-0.990 \quad 4.880 \quad 0.660 \quad 0.710$

TOMB GPS $240.934 \quad 43.585 \quad-1.870 \quad 1.120 \quad 0.720 \quad 0.760$

TONA GPS $240.548 \quad 48.694 \quad 1.620 \quad 0.440 \quad 0.870 \quad 0.940$

TRIA_GPS $236.433 \quad 44.185 \quad 3.000 \quad 9.080 \quad 0.560 \quad 0.610$

TRND_GPS $235.849 \quad 41.054 \quad 3.373 \quad 16.454 \quad 0.392 \quad 0.374$

TSWY_GPS $249.403 \quad 43.674-1.326-0.802 \quad 0.505 \quad 0.507$

TUCK_GPS $236.597 \quad 48.140 \quad 7.470 \quad 4.530 \quad 1.380 \quad 1.190$

TURN_GPS $241.993 \quad 42.923 \quad-2.610 \quad 0.290 \quad 0.880 \quad 0.770$

TWIN_GPS $236.996 \quad 44.325 \quad 2.660 \quad 5.630 \quad 0.850 \quad 0.860$

U701_GPS $242.417 \quad 44.259 \quad-4.910 \quad 3.400 \quad 2.000 \quad 1.480$

U727_GPS $236.453 \quad 44.594 \quad 4.030 \quad 8.630 \quad 0.570 \quad 0.670$

U73H_GPS 239.782 $44.631-1.390 \quad 1.910 \quad 0.800 \quad 0.820$

U76A_GPS $244.269 \quad 43.131-0.120-0.960 \quad 0.970 \quad 0.900$

UCD1_GPS $238.249 \quad 38.536-11.530 \quad 7.397 \quad 0.418 \quad 0.415$

UCLU_GPS $234.458 \quad 48.926 \quad 9.010 \quad 7.640 \quad 0.310 \quad 0.300$

V162_GPS $244.442 \quad 43.490 \quad-2.570 \quad 0.260 \quad 1.240 \quad 0.850$

V357_GPS 238.973 42.393 -3.400 4.720 $0.690 \quad 0.820$

V546_GPS 238.122 $42.574 \quad-1.940 \quad 6.070 \quad 0.610 \quad 0.670$

V696_GPS $241.385 \quad 42.450 \quad-2.240 \quad 0.740 \quad 0.870 \quad 0.750$

VANC_GPS $237.256 \quad 45.672 \quad 5.850 \quad 4.900 \quad 1.030 \quad 1.120$

VLBI_GPS $236.513 \quad 48.390 \quad 5.213 \quad 4.541 \quad 0.367 \quad 0.358$

WALA_GPS $241.717 \quad 46.088 \quad-0.100 \quad 0.310 \quad 0.500 \quad 0.520$

WAS2_GPS $234.703 \quad 49.752 \quad 3.350 \quad 4.960 \quad 1.750 \quad 1.660$

WELK_GPS $236.417 \quad 43.644 \quad 0.680 \quad 8.880 \quad 1.540 \quad 1.850$

WHD1_GPS $237.304 \quad 48.313 \quad 5.300 \quad 4.390 \quad 0.840 \quad 0.900$

WHEY_GPS $237.965 \quad 45.376 \quad 1.070 \quad 5.570 \quad 0.510 \quad 0.560$

WHIT_GPS $224.778 \quad 60.751 \quad 0.640 \quad 2.978 \quad 0.461 \quad 0.458$

WILD_GPS $241.629 \quad 40.015 \quad-5.460 \quad 1.500 \quad 1.330 \quad 0.930$

WILL_GPS $237.832 \quad 52.237 \quad-0.604 \quad 0.581 \quad 0.435 \quad 0.435$

WILS_GPS $239.479 \quad 47.012 \quad 2.780 \quad 1.420 \quad 0.590 \quad 0.550$

WORD_GPS $237.232 \quad 48.141 \quad 4.820 \quad 3.690 \quad 0.640 \quad 0.620$

WSLR_GPS $237.079 \quad 50.127 \quad 2.040 \quad 2.460 \quad 0.380 \quad 0.390$

X537_GPS 235.943 46.516 8.810 $11.060 \quad 0.560 \quad 0.620$

Y109_GPS $238.865 \quad 45.233 \quad 0.720 \quad 2.500 \quad 0.460 \quad 0.480$

Y129_GPS $242.284 \quad 42.013 \quad-3.890 \quad 1.490 \quad 1.050 \quad 1.070$ 
$\begin{array}{lllllll}\text { Y } 405 \text { GPS } & 243.709 & 45.656 & 2.290 & 0.950 & 0.860 & 0.930\end{array}$ $\begin{array}{lllllll}\text { Y502 GPS } & 238.634 & 44.823 & 0.930 & 3.120 & 0.570 & 0.710\end{array}$ Y683_GPS 236.781 $44.689 \quad 3.610 \quad 7.360 \quad 1.540 \quad 0.820$ YALE_GPS $237.682 \quad 46.026 \quad 2.130 \quad 4.710 \quad 0.380 \quad 0.400$ YAMB_GPS $236.861 \quad 45.070 \quad 3.150 \quad 6.650 \quad 0.530 \quad 0.560$ YOUB_GPS $235.738 \quad 48.901 \quad 5.880 \quad 5.240 \quad 0.440 \quad 0.430$ Z231_GPS $242.667 \quad 47.9692 .400 \quad-0.050 \quad 1.130 \quad 1.380$ Z264_GPS $242.112 \quad 48.541 \quad 0.840 \quad 0.720 \quad 0.440 \quad 0.460$ Z478_GPS $236.888 \quad 47.033 \quad 5.550 \quad 6.670 \quad 0.470 \quad 0.510$ $\begin{array}{llllllll}\text { RVAL_GPS } & 244.598 & 35.142 & -2.375 & 0.701 & 0.709 & 0.707\end{array}$

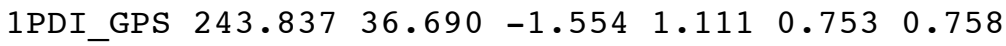
$\begin{array}{lllllll}\text { SHOS_GPS } & 243.739 & 36.943 & -1.685 & 1.663 & 0.752 & 0.753\end{array}$ SILV_GHT $243.709 \quad 35.397 \quad-5.139 \quad 0.291 \quad 1.320 \quad 1.300$ FUNE GPS $243.525 \quad 36.397 \quad-3.139 \quad 1.452 \quad 0.695 \quad 0.700$ P_42_GHT $243.448 \quad 35.426 \quad-4.634 \quad 1.925 \quad 1.420 \quad 1.370$ BLAK_GPS $243.425 \quad 36.809 \quad-3.124 \quad 1.908 \quad 1.370 \quad 1.370$ $\begin{array}{lllllll}\text { CLAI_GPS } & 243.319 & 36.889 & -4.246 & 1.507 & 1.370 & 1.380\end{array}$ $\begin{array}{llllllll}\text { P16X_GPS } & 243.254 & 36.828 & -3.074 & 2.929 & 0.779 & 0.797\end{array}$ F23X_GPS $243.138 \quad 36.858 \quad-3.431 \quad 2.976 \quad 0.750 \quad 0.766$ MO93_GPS $243.071 \quad 36.792 \quad-4.106 \quad 2.720 \quad 0.758 \quad 0.775$ BM8Z_GPS $243.022 \quad 36.724 \quad-3.242 \quad 2.544 \quad 0.718 \quad 0.725$ GS36_GPS $242.958 \quad 35.168-10.024 \quad 10.569 \quad 1.350 \quad 1.360$ SCTY_GPS $242.863 \quad 37.218 \quad-3.179 \quad 2.008 \quad 1.040 \quad 1.020$ STOV_GPS $242.853 \quad 36.606 \quad-4.935 \quad 3.430 \quad 0.757 \quad 0.763$ GS13_GPS $242.851 \quad 35.522 \quad-6.988 \quad 8.215 \quad 0.698 \quad 0.698$ GS49_GHT $242.837 \quad 35.375 \quad-6.795 \quad 11.520 \quad 1.300 \quad 1.290$ PANA GPS $242.826 \quad 36.294 \quad-4.505 \quad 3.881 \quad 0.678 \quad 0.682$ GS07_GPS $242.821 \quad 36.035 \quad-6.044 \quad 5.207 \quad 0.698 \quad 0.700$ GS14_GPS $242.801 \quad 35.615 \quad-7.484 \quad 7.983 \quad 0.700 \quad 0.702$ $\begin{array}{lllllll}\text { RAIN_GPS } & 242.792 & 34.975 & -9.224 & 12.368 & 0.723 & 0.725\end{array}$ $\begin{array}{lllllll}\text { G165 GPS } & 242.788 & 36.543 & -4.426 & 3.724 & 0.763 & 0.770\end{array}$ X137_GPS 242.720 $36.402 \quad-5.299 \quad 4.383 \quad 0.733 \quad 0.733$ NEV1_GPS $242.714 \quad 37.061 \quad-3.346 \quad 2.691 \quad 1.010 \quad 0.990$ GS25_GPS $242.711 \quad 35.913 \quad-6.568 \quad 6.654 \quad 0.698 \quad 0.698$ M137_GPS 242.700 $36.349 \quad-4.904 \quad 6.120 \quad 0.758 \quad 0.766$ GS47_GHT $242.683 \quad 35.214 \quad-9.331 \quad 12.163 \quad 1.320 \quad 1.320$ TRN1_GPS $242.672 \quad 35.813 \quad-6.676 \quad 6.721 \quad 0.709 \quad 0.711$ $\begin{array}{llllllll}\text { GS12_GPS } & 242.667 & 35.434 & -8.219 & 10.032 & 0.704 & 0.705\end{array}$ GRAP_GPS $242.640 \quad 36.992 \quad-3.753 \quad 2.477 \quad 1.030 \quad 1.010$ GS20_GPS $242.599 \quad 35.769 \quad-7.725 \quad 8.200 \quad 0.700 \quad 0.700$ 13DD_GPS $242.576 \quad 36.340 \quad-6.362 \quad 6.102 \quad 0.747 \quad 0.763$ GS27_GPS $242.546 \quad 36.053 \quad-7.043 \quad 6.623 \quad 0.728 \quad 0.728$ GS48_GPS $242.541 \quad 35.584 \quad-8.703 \quad 10.695 \quad 1.340 \quad 1.360$ HUNT GPS $242.521 \quad 36.572 \quad-5.277 \quad 4.147 \quad 1.060 \quad 1.050$ GS24_GPS $242.518 \quad 35.925 \quad-8.409 \quad 7.081 \quad 0.709 \quad 0.711$ TINP_GPS $242.500 \quad 36.865 \quad-3.966 \quad 3.879 \quad 1.020 \quad 1.010$ FMTH_GPS $242.495 \quad 35.213 \quad-9.901 \quad 12.249 \quad 1.320 \quad 1.320$ JACK_GPS $242.460 \quad 36.532 \quad-5.742 \quad 4.442 \quad 1.060 \quad 1.050$ GS26_GCO $242.458 \quad 35.740 \quad-8.598 \quad 8.733 \quad 1.170 \quad 1.170$ GS43_GPS $242.454 \quad 36.067 \quad-7.882 \quad 6.753 \quad 1.280 \quad 1.280$ TEAK_GPS $242.454 \quad 36.759 \quad-4.609 \quad 3.783 \quad 1.040 \quad 1.010$ L166_GPS $242.450 \quad 36.279 \quad-6.633 \quad 5.925 \quad 0.760 \quad 0.781$ GS17_GPS $242.443 \quad 35.569 \quad-9.729 \quad 10.485 \quad 0.700 \quad 0.702$ GS45_GPS $242.443 \quad 36.002 \quad-7.566 \quad 7.524 \quad 1.280 \quad 1.290$ FLAT_GPS $242.439 \quad 36.519 \quad-5.476 \quad 3.964 \quad 1.060 \quad 1.060$ T19S_GPS $242.417 \quad 36.232 \quad-7.588 \quad 6.121 \quad 0.768 \quad 0.789$ $\begin{array}{lllllll}\text { GS11_GPS } & 242.415 & 35.429 & -9.122 & 12.012 & 0.747 & 0.750\end{array}$ GS50_GPS $242.409 \quad 35.083 \quad-10.772 \quad 13.997 \quad 1.330 \quad 1.320$ LEEF_GPS $242.388 \quad 36.497 \quad-6.008 \quad 5.568 \quad 1.060 \quad 1.070$ GS42_GPS $242.368 \quad 36.107 \quad-8.190 \quad 5.990 \quad 1.290 \quad 1.300$ 
GS28_GPS $242.359 \quad 36.307 \quad-6.582 \quad 5.536 \quad 0.673 \quad 0.669$ GS34_GPS 242.328 $36.094 \quad-9.959 \quad 6.074 \quad 1.140 \quad 1.120$ 6813_GPS 242.325 36.150 $-8.914 \quad 5.348 \quad 0.731 \quad 0.738$ TABL_GPS $242.322 \quad 34.382 \quad-20.099 \quad 19.628 \quad 0.721 \quad 0.721$ JOBU_GPS $242.308 \quad 35.337 \quad-8.855 \quad 12.474 \quad 0.686 \quad 0.687$

MDAY_GPS $242.294 \quad 34.743 \quad-13.129 \quad 15.444 \quad 0.737 \quad 0.733$ GS16_GPS $242.294 \quad 35.470 \quad-9.687 \quad 11.689 \quad 0.700 \quad 0.702$ GS19_GPS $242.260 \quad 35.660 \quad-9.932 \quad 11.302 \quad 0.702 \quad 0.702$ GS35_GPS 242.244 36.217 $\quad-7.475 \quad 6.811 \quad 1.150 \quad 1.130$ $\begin{array}{lllllllll}\text { CERR_GPS } 242.213 & 36.538 & -7.687 & 6.259 & 0.726 & 0.742\end{array}$ $\begin{array}{llllllll}\text { HOLC_GNR } 242.155 & 34.458 & -18.278 & 21.088 & 0.698 & 0.698\end{array}$ TTAP_GPS $242.136 \quad 34.985 \quad-11.569 \quad 15.029 \quad 0.702 \quad 0.702$ GS18_GPS $242.130 \quad 35.584 \quad-10.971 \quad 11.220 \quad 0.702 \quad 0.704$ FORK GGE 242.116 36.062 $\quad$-7.775 8.371 $1.100 \quad 1.110$ GS09_GPS 242.102 35.115 $-10.842 \quad 14.191 \quad 0.707 \quad 0.709$ GS04_GPS 242.093 $36.204 \quad-8.833 \quad 8.021 \quad 0.696 \quad 0.698$ BM25_GPS 242.056 $36.045 \quad-9.030 \quad 10.689 \quad 0.752 \quad 0.771$ GS15_GPS 242.039 35.426 $-11.198 \quad 11.820 \quad 0.705 \quad 0.707$ GS22 GPS $242.031 \quad 35.845 \quad-9.902 \quad 11.221 \quad 0.735 \quad 0.737$ WAUC_GPS 242.013 37.092 $\quad-6.940 \quad 6.690 \quad 1.340 \quad 1.340$ GS03_GPS $241.965 \quad 35.668 \quad-10.929 \quad 11.659 \quad 0.733 \quad 0.738$ FISH_GPS $241.954 \quad 37.737 \quad-4.824 \quad 5.324 \quad 1.170 \quad 1.170$ GS01_GPS $241.918 \quad 35.225 \quad-11.608 \quad 13.572 \quad 0.705 \quad 0.705$ GS02_GPS $241.900 \quad 35.494 \quad-11.460 \quad 11.698 \quad 0.700 \quad 0.702$ RITA_GPS $241.899 \quad 36.914 \quad-8.215 \quad 6.223 \quad 0.691 \quad 0.693$ BAMA_GPS $241.881 \quad 36.603 \quad-8.723 \quad 8.254 \quad 0.696 \quad 0.698$ 3188 GPS $241.868 \quad 36.466 \quad-9.532 \quad 9.371 \quad 1.010 \quad 0.980$ KMED_GPS $241.864 \quad 36.023 \quad-9.991 \quad 10.155 \quad 0.680 \quad 0.682$ WSTG_GPS $241.848 \quad 37.271 \quad-7.143 \quad 5.337 \quad 0.716 \quad 0.718$ TROP GPS $241.794 \quad 34.992 \quad-12.529 \quad 14.860 \quad 0.805 \quad 0.802$ WMTN_GPS $241.764 \quad 37.572 \quad-5.130 \quad 5.167 \quad 0.705 \quad 0.709$ ABER_GPS 241.712 36.979 $-8.809 \quad 7.734 \quad 1.050 \quad 1.030$ OVRO_GPS $241.706 \quad 37.233 \quad-7.627 \quad 7.205 \quad 0.682 \quad 0.684$ DEER_GPS $241.492 \quad 35.086 \quad-12.328 \quad 14.733 \quad 0.700 \quad 0.700$ 0614_GPS $241.412 \quad 35.745 \quad-11.829 \quad 10.553 \quad 0.689 \quad 0.689$ SPRN_GPS $241.273 \quad 36.185-11.578 \quad 10.421 \quad 0.841 \quad 0.735$ ALRT_PBO 297.660 82.494 $-0.083-1.667 \quad 0.900 \quad 0.600$ $\begin{array}{lllllll}\mathrm{SCH} 2 \text { - } \mathrm{PBO} & 293.167 & 54.832 & 0.846 & 0.665 & 0.474 & 0.524\end{array}$ THU3_PBO 291.175 $76.537-0.883-0.439 \quad 0.200 \quad 0.200$ $\begin{array}{llllllll}\mathrm{NRC} 1 & -\mathrm{PBO} & 284.376 & 45.454 & 0.362 & -0.560 & 0.474 & 0.418\end{array}$ GOGA_PBO $276.527 \quad 33.415 \quad-3.377-1.220 \quad 1.900 \quad 1.800$ JFWS_PBO 269.752 $42.914 \quad-3.912 \quad-5.761 \quad 1.600 \quad 1.600$ RESO_PBO 265.106 74.691 $-0.048-0.251 \quad 1.1001 .900$ KSU1_PBO 263.391 39.101 $-2.239-1.010 \quad 1.300 \quad 1.300$ WMOK_PBO 261.219 34.738 $-0.996-1.457 \quad 1.1001 .100$ P040_PBO 257.313 38.071 $-0.475-1.463 \quad 0.400 \quad 1.000$ P039_PBO 256.846 36.448 $\quad-0.934 \quad-0.052 \quad 0.500 \quad 0.400$ P044_PBO 256.778 $40.172 \quad-0.698 \quad-0.350 \quad 0.400 \quad 0.300$ P038_PBO 256.593 34.147 -1.217 -0.346 $0.500 \quad 0.500$ P043_PBO 255.814 43.881 $-0.962-1.027 \quad 1.200 \quad 0.700$ P054_PBO 255.559 45.846 $-0.689-0.721 \quad 1.400 \quad 0.900$ P055_PBO 255.315 47.117 $-0.642-1.215 \quad 1.200 \quad 0.800$ P042_PBO 255.089 42.052 $-1.030-0.010 \quad 0.400 \quad 0.300$ P037_PBO 254.895 38.422 $-0.764-0.105 \quad 0.300 \quad 0.400$ P035_PBO 254.816 34.601 $-1.402-0.103 \quad 0.500 \quad 0.500$ P041_PBO 254.806 39.949 $-0.608-0.203 \quad 0.400 \quad 0.300$ P036_PBO 254.706 36.420 $\quad-1.637 \quad 0.299 \quad 0.600 \quad 0.300$ RG17_PBO 254.330 39.762 -3.115 -0.292 $1.700 \quad 1.700$ P123_PBO 254.089 36.635 -0.730 $-0.486 \quad 1.100 \quad 0.800$ P034_PBO 253.541 34.946 $-1.091 \quad 0.027 \quad 0.300 \quad 0.300$ 
$\begin{array}{lllllllll}\text { SC01_PBO } & 253.033 & 34.068 & -0.824 & -1.161 & 0.800 & 0.700\end{array}$ $\begin{array}{llllllll}\mathrm{P} 052 & \mathrm{PBO} & 252.981 & 47.375 & -0.536 & -0.959 & 0.900 & 1.000\end{array}$ $\begin{array}{lllllllll}\text { P026_PBO } 252.805 & 32.659 & -1.574 & 0.945 & 0.400 & 0.700\end{array}$ P032_PBO 252.744 41.742 $-0.545-0.254 \quad 0.800 \quad 0.500$ P033_PBO 252.612 43.953 $-0.564-1.150 \quad 0.600 \quad 0.500$ P107_PBO 252.120 35.132 $\quad-0.987 \quad 0.461 \quad 1.200 \quad 0.900$

P028_PBO 252.092 36.032 -0.955 -0.338 0.5001 .100 $\begin{array}{lllllll}\text { P031_PBO 252.091 39.515 } 0.772 & -1.638 & 1.200 & 0.800\end{array}$

P051_PBO 251.454 45.807 $-0.298-1.223 \quad 0.600 \quad 0.500$ P012_PBO 250.666 38.097 -1.383 -0.504 $1.000 \quad 0.800$ P718_PBO 250.624 44.753 -2.039 -1.503 1.3001 .400 P011_PBO 250.481 36.150 -0.753 -0.600 $1.200 \quad 0.800$ $\begin{array}{llllllll}\text { BLW2_PBO } 250.442 & 42.767 & -1.912 & -0.099 & 0.300 & 0.300\end{array}$ $\begin{array}{llllllll}\mathrm{P} 722 & -\mathrm{PBO} & 250.429 & 45.457 & -0.113 & -0.898 & 0.900 & 0.500\end{array}$ P715_PBO 250.310 43.501 $-0.886-0.296 \quad 1.600 \quad 1.700$ P717_PBO 250.103 44.485 $-0.150-1.391 \quad 1.200 \quad 1.200$ P721_PBO 249.998 45.003 -0.731 $-0.788 \quad 0.700 \quad 0.700$ P015_PBO 249.991 34.264 -1.722 $-0.188 \quad 0.500 \quad 0.400$ P709_PBO 249.714 44.392 $-0.654-0.381 \quad 1.000 \quad 0.800$ WLWY_PBO $249.713 \quad 44.640 \quad 4.755-4.781 \quad 0.500 \quad 0.300$ P720_PBO 249.694 44.943 $-0.834-0.381 \quad 0.700 \quad 0.600$ P716_PBO 249.488 44.718 $-10.443 \quad 0.924 \quad 0.900 \quad 0.600$ P030_PBO 249.487 41.750 -1.152 -0.676 $1.000 \quad 0.500$ $\begin{array}{llllllll}\mathrm{P} 461[\mathrm{PBO} & 249.241 & 45.354 & 0.580 & -0.970 & 0.900 & 0.500\end{array}$ P711_PBO 249.139 44.636 $11.353 \quad 3.432 \quad 0.900 \quad 0.500$ P049_PBO 249.094 47.350 $0.353-0.967 \quad 1.200 \quad 1.400$

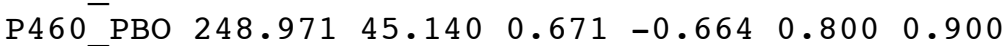
$\begin{array}{lllllllll}\mathrm{P} 680 \mathrm{PBO} & 248.901 & 44.598 & 0.651 & -2.462 & 0.600 & 0.800\end{array}$ P101_PBO 248.764 41.692 $-0.156-0.759 \quad 0.900 \quad 0.500$ P050_PBO 248.752 48.809 $0.806-1.259 \quad 0.900 \quad 0.800$ P119_PBO 248.742 $40.732 \quad-0.392 \quad-0.958 \quad 1.100 \quad 1.300$ P118_PBO 248.650 40.635 -1.095 $-0.756 \quad 0.600 \quad 0.600$ P089_PBO 248.585 $40.807 \quad-1.089 \quad 0.145 \quad 0.400 \quad 0.300$ P360_PBO 248.549 $44.318-1.961 \quad-2.754 \quad 0.500 \quad 0.500$ P684_PBO $248.550 \quad 43.919-2.175-1.654 \quad 0.500 \quad 0.400$ P112_PBO 248.550 39.817 $-0.825 \quad-0.354 \quad 0.500 \quad 0.600$ BBID_PBO 248.474 44.185 -2.566 $-2.452 \quad 0.600 \quad 0.800$ HWUT_PBO $248.435 \quad 41.607 \quad-0.760 \quad 0.049 \quad 1.600 \quad 1.600$ $\begin{array}{llllllll}\mathrm{P} 110 & \text { PBO } & 248.429 & 39.715 & 0.171 & -1.451 & 0.700 & 0.500\end{array}$ $\begin{array}{llllllll}\text { MPUT } & -1 B O & 248.366 & 40.016 & -2.519 & 1.051 & 0.600 & 0.400\end{array}$ P088_PBO 248.277 $40.772 \quad-0.591-1.347 \quad 1.300 \quad 1.400$ P117_PBO 248.249 40.435 $-3.004-2.247 \quad 1.400 \quad 1.600$ $\begin{array}{llllllll}\mathrm{P} 719 & \text { PBO } 248.211 & 45.218 & 0.772 & -1.646 & 1.400 & 1.500\end{array}$ P108_PBO 248.055 39.589 -1.735 -0.742 $0.500 \quad 0.600$ $\begin{array}{lllllllll}\text { SPIC_PBO } & 247.873 & 39.306 & -2.946 & 0.862 & 0.300 & 0.200\end{array}$ P106_PBO 247.738 39.459 $-2.741-0.535 \quad 1.100 \quad 1.100$ P086_PBO 247.718 $40.649 \quad-2.498 \quad 0.966 \quad 0.400 \quad 0.400$ P122_PBO 247.668 $41.635-2.262-0.833 \quad 0.300 \quad 0.200$ P016_PBO 247.639 40.078 -2.719 $-0.232 \quad 1.4001 .100$ FERN_PBO 247.545 $35.342 \quad-2.390 \quad 0.470 \quad 0.300 \quad 0.200$ P105_PBO 247.496 39.388 $-2.744 \quad 0.471 \quad 0.500 \quad 0.700$

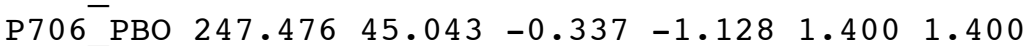
P114_PBO 247.472 $40.634 \quad-2.699-0.328 \quad 0.800 \quad 0.400$ P045_PBO 247.383 45.383 $-0.325-1.826 \quad 1.200 \quad 1.300$ P057_PBO 247.377 41.757 -2.758 $-0.426 \quad 0.300 \quad 0.300$

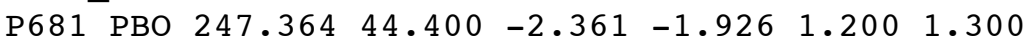
$\begin{array}{lllllll}\mathrm{P} 121 & \text { PBO } 247.302 & 41.803 & -2.857 & -0.824 & 0.300 & 0.300\end{array}$ P104_PBO 247.283 39.186 -1.452 -1.024 $1.600 \quad 1.100$ P675_PBO 247.281 42.212 $-3.342-1.424 \quad 1.200 \quad 1.200$ P678_PBO $247.195 \quad 43.449-2.197 \quad-1.522 \quad 1.600 \quad 1.300$ 


$$
\begin{aligned}
& \begin{array}{llllllll}
\text { P111 PBO } & 246.988 & 41.817 & -2.657 & -1.717 & 1.200 & 1.200
\end{array} \\
& \text { P084_PBO } 246.946 \quad 40.494 \quad-3.606 \quad-0.916 \quad 1.100 \quad 1.100 \\
& \text { P113_PBO } 246.722 \quad 40.671 \quad-3.200-1.111 \quad 1.000 \quad 1.200 \\
& \text { P046_PBO } 246.668 \quad 47.030 \quad 0.433 \quad-2.009 \quad 1.300 \quad 1.400 \\
& \begin{array}{lllllll}
\text { P082_PBO } & 246.495 & 39.269 & -2.852 & -0.505 & 1.300 & 1.300
\end{array} \\
& \text { P677_PBO } 246.132 \quad 42.879 \quad-2.522 \quad-1.297 \quad 1.000 \quad 1.200 \\
& \begin{array}{lllllll}
\text { P081_PBO } & 246.129 & 39.067 & -2.960 & -0.297 & 1.100 & 1.100
\end{array} \\
& \text { P354_PBO } 246.021 \quad 44.109 \quad-1.277 \quad-1.494 \quad 1.200 \quad 1.200 \\
& \text { P003_PBO } 245.995 \quad 32.723 \quad-3.287 \quad 0.106 \quad 0.700 \quad 0.700 \\
& \begin{array}{llllllll}
\text { GNPS_PBO } & 245.811 & 34.309 & -2.332 & 2.911 & 0.200 & 0.200
\end{array} \\
& \text { P080_PBO } 245.723 \quad 39.119 \quad-2.160 \quad-0.287 \quad 1.600 \quad 1.700 \\
& \text { P623_PBO } 245.401 \quad 34.189 \quad-3.637 \quad 0.420 \quad 0.400 \quad 0.400 \\
& \begin{array}{lllllll}
\text { GMPK_PBO } & 245.173 & 33.051 & -3.478 & 2.626 & 0.300 & 0.200
\end{array} \\
& \begin{array}{lllllll}
\text { IID2 } 2 \text { PBO } & 244.968 & 32.706 & -3.893 & 2.321 & 0.570 & 0.616
\end{array} \\
& \begin{array}{lllllll}
\text { IMPS PBO } & 244.855 & 34.158 & -2.556 & 0.639 & 0.589 & 0.572
\end{array} \\
& \text { P626_PBO } 244.762 \quad 35.291 \quad-3.400 \quad 0.0350 .600 \quad 0.500 \\
& \begin{array}{llllllll}
\text { P005 PBO } & 244.721 & 39.910 & -2.935 & -1.064 & 1.500 & 1.600
\end{array} \\
& \text { P509_PBO } 244.706 \quad 32.891 \quad-1.585 \quad 3.137 \quad 0.600 \quad 0.600 \\
& \text { P511_PBO } 244.704 \quad 33.887 \quad-4.550 \quad 0.637 \quad 0.400 \quad 0.400 \\
& \begin{array}{llllllll}
\text { P500_PBO } 244.700 & 32.690 & -6.392 & 8.937 & 0.500 & 0.500
\end{array} \\
& \text { P510_PBO } 244.657 \quad 33.144 \quad-3.976 \quad 1.538 \quad 0.600 \quad 0.600 \\
& \text { P622_PBO } 244.634 \quad 35.163 \quad-3.605 \quad 0.038 \quad 0.600 \quad 0.500 \\
& \text { P501_PBO } 244.602 \quad 32.876 \quad-4.686 \quad 6.439 \quad 0.400 \quad 0.400 \\
& \begin{array}{llllllll}
\text { P502_PBO } 244.578 & 32.982 & -4.682 & 3.940 & 0.500 & 0.600
\end{array} \\
& \begin{array}{llllllll}
\text { P508_PBO } 244.571 & 33.248 & -4.873 & 1.940 & 0.400 & 0.400
\end{array} \\
& \begin{array}{llllllll}
\text { P } 499 \text { PBO } & 244.512 & 32.980 & -6.783 & 5.041 & 0.600 & 0.700
\end{array} \\
& \begin{array}{lllllll}
\text { IVCO PBO } & 244.493 & 32.829 & -14.864 & 20.255 & 0.626 & 0.534
\end{array} \\
& \begin{array}{llllllll}
\text { P506 PBO } 244.490 & 33.081 & -6.579 & 4.042 & 0.800 & 0.800
\end{array} \\
& \text { P076_PBO } 244.487 \quad 39.536 \quad-2.349 \quad-0.458 \quad 1.500 \quad 1.600 \\
& \text { GLRS_PBO } 244.479 \quad 33.275 \quad-6.872 \quad 1.742 \quad 0.300 \quad 0.500 \\
& \text { P621_PBO } 244.456 \quad 35.473 \quad-3.995 \quad 0.042 \quad 0.600 \quad 0.500 \\
& \begin{array}{llllllll}
\text { P102_PBO } & 244.444 & 39.925 & -2.635 & 0.243 & 1.500 & 1.500
\end{array} \\
& \begin{array}{lllllll}
\text { P498_PBO } 244.430 & 32.898 & -15.286 & 21.843 & 0.500 & 0.500
\end{array} \\
& \begin{array}{lllllll}
\text { P497_PBO } 244.423 & 32.835 & -16.488 & 22.143 & 0.500 & 0.600
\end{array} \\
& \begin{array}{lllllllll}
\text { P496_PBO } 244.404 & 32.751 & -23.391 & 28.044 & 0.500 & 0.900
\end{array} \\
& \text { P507_PBO } 244.388 \quad 33.200 \quad-8.963 \quad 0.209 \quad 0.602 \quad 0.644 \\
& \begin{array}{llllllll}
\text { P495_PBO } & 244.372 & 33.045 & -11.281 & 15.244 & 0.400 & 0.400
\end{array} \\
& \text { HNPS_PBO } 244.365 \quad 33.705 \quad-5.858 \quad 2.745 \quad 0.200 \quad 0.300 \\
& \text { P611_PBO } 244.350 \quad 35.205 \quad-4.805 \quad 0.0450 .900 \quad 1.000 \\
& \begin{array}{lllllll}
\text { GMRC PBO } & 244.340 & 34.784 & -3.720 & 1.045 & 0.200 & 0.200
\end{array} \\
& \begin{array}{lllllll}
\text { P505_PBO } & 244.313 & 33.424 & -5.868 & 3.346 & 1.000 & 0.900
\end{array} \\
& \begin{array}{llllllll}
\text { P494_PBO } & 244.268 & 32.760 & -22.791 & 26.547 & 0.600 & 0.500
\end{array} \\
& \text { CRRS_PBO } 244.265 \quad 33.070 \quad-14.236 \quad 18.442 \quad 0.596 \quad 0.577 \\
& \text { P610_PBO } 244.236 \quad 34.426 \quad-3.533 \quad 0.848 \quad 1.100 \quad 1.100 \\
& \text { P504_PBO } 244.234 \quad 33.516 \quad-8.565 \quad 4.148 \quad 0.500 \quad 0.500 \\
& \text { P607_PBO } 244.179 \quad 33.741 \quad-6.357 \quad 2.849 \quad 0.900 \quad 1.000 \\
& \text { P075_PBO } 244.111 \quad 39.374 \quad 0.643 \quad-5.650 \quad 1.500 \quad 1.600 \\
& \text { I40A_PBO } 244.089 \quad 34.727 \quad-4.023 \quad 1.551 \quad 0.400 \quad 0.500 \\
& \text { OPBL_PBO } 244.082 \quad 34.370 \quad-2.935 \quad 2.651 \quad 0.300 \quad 0.200 \\
& \text { SLMS_PBO } 244.022 \quad 33.292 \quad-13.573 \quad 16.453 \quad 0.200 \quad 0.200 \\
& \text { BEMT_PBO } 244.002 \quad 34.001 \quad-5.948 \quad 5.653 \quad 0.300 \quad 0.200 \\
& \text { P074_PBO } 243.950 \quad 39.546 \quad-2.751 \quad-0.646 \quad 1.400 \quad 1.500 \\
& \text { BMHL_PBO } 243.947 \quad 34.251 \quad-3.740 \quad 4.454 \quad 0.200 \quad 0.200 \\
& \begin{array}{lllllll}
\text { BKAP_PBO } & 243.920 & 35.287 & -4.903 & 1.155 & 0.200 & 0.200
\end{array} \\
& \text { P601_PBO } 243.920 \quad 33.959 \quad-6.550 \quad 5.455 \quad 0.900 \quad 1.000 \\
& \begin{array}{lllllll}
\text { OPCP PBO } & 243.917 & 34.367 & -2.836 & 4.355 & 0.200 & 0.200
\end{array} \\
& \begin{array}{llllllll}
\text { USGC_PBO } & 243.915 & 33.030 & -23.913 & 24.402 & 0.570 & 0.574
\end{array} \\
& \text { P618_PBO } 243.896 \quad 35.142 \quad-4.709 \quad 0.155 \quad 0.500 \quad 0.600 \\
& \text { NBPS_PBO } 243.852 \quad 34.509 \quad-3.431 \quad 3.256 \quad 0.200 \quad 0.300 \\
& \begin{array}{llllllll}
\text { OPCX_PBO } & 243.851 & 34.430 & -2.934 & 4.156 & 0.300 & 0.200
\end{array}
\end{aligned}
$$


$\begin{array}{llllllll}\text { P066_PBO } & 243.830 & 32.617 & -27.898 & 26.957 & 0.300 & 0.300\end{array}$ $\begin{array}{llllllll}\text { LDSW PBO } & 243.791 & 34.699 & -6.025 & 2.158 & 0.300 & 0.500\end{array}$ P600_PBO $243.788 \quad 33.866 \quad-9.654 \quad 9.558 \quad 0.300 \quad 0.400$ P491_PBO 243.773 $33.575 \quad-15.164 \quad 14.858 \quad 0.400 \quad 0.400$ $\begin{array}{llllllll}\text { SDHL_PBO } & 243.721 & 34.255 & -5.921 & 5.183 & 0.707 & 0.629\end{array}$ P087_PBO $243.721 \quad 40.363 \quad-2.523 \quad 0.659 \quad 1.500 \quad 1.600$ OPRD_PBO $243.708 \quad 34.533 \quad-6.431 \quad 5.860 \quad 0.200 \quad 0.200$ $\begin{array}{llllllll}\text { SHOS_PBO } & 243.701 & 35.971 & -4.480 & 1.160 & 0.200 & 0.200\end{array}$ $\begin{array}{llllllll}\text { OPCL_PBO } & 243.695 & 34.428 & -5.735 & 7.860 & 0.200 & 0.300\end{array}$ $\begin{array}{llllllll}\mathrm{P} 486 \_ \text {PBO } & 243.678 & 33.260 & -23.476 & 22.060 & 0.300 & 0.300\end{array}$ $\begin{array}{llllllll}\text { CDMT_PBO } & 243.664 & 34.829 & -7.321 & 2.961 & 0.200 & 0.200\end{array}$ $\begin{array}{llllllll}\text { P480_PBO } & 243.651 & 32.976 & -27.186 & 25.261 & 0.300 & 0.300\end{array}$ $\begin{array}{lllllll}\text { CTMS_PBO } & 243.630 & 34.124 & -7.045 & 11.662 & 0.200 & 0.200\end{array}$ $\begin{array}{llllllll}\text { P485 PBO } & 243.591 & 33.210 & -26.278 & 24.363 & 1.200 & 1.200\end{array}$ P073 PBO $243.576 \quad 39.501 \quad-2.954 \quad 0.763 \quad 1.400 \quad 1.500$ $\begin{array}{lllllll}\text { P490_PBO } & 243.574 & 33.523 & -18.172 & 17.873 & 0.607 & 0.620\end{array}$ AGMT_PBO $243.571 \quad 34.594 \quad-8.129 \quad 7.763 \quad 0.200 \quad 0.200$ HCMN_PBO $243.570 \quad 34.755 \quad-8.623 \quad 4.463 \quad 0.200 \quad 0.200$

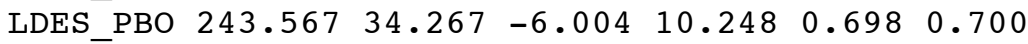
P599_PBO $243.463 \quad 34.217 \quad-8.943 \quad 13.165 \quad 1.100 \quad 1.100$ $\begin{array}{lllllll}\text { P483_PBO } 243.431 & 33.059 & -26.984 & 26.066 & 0.300 & 0.300\end{array}$ P617_PBO 243.428 35.321 $\quad-5.004 \quad 2.866 \quad 1.100 \quad 1.100$ $\begin{array}{llllllll}\mathrm{P} 484 \text { PBO } & 243.379 & 33.376 & -24.473 & 22.967 & 0.400 & 0.400\end{array}$ RDMT_PBO $243.375 \quad 34.644 \quad-10.928 \quad 8.768 \quad 0.300 \quad 0.200$ $\begin{array}{llllllll}\text { P 482_PBO } & 243.329 & 33.240 & -26.078 & 24.969 & 0.400 & 0.300\end{array}$ $\begin{array}{llllllll}\text { DSSC_PBO } & 243.288 & 33.733 & -18.360 & 19.870 & 0.400 & 0.300\end{array}$ P085 PBO $243.264 \quad 40.495 \quad-2.920 \quad-0.630 \quad 1.500 \quad 1.500$ $\begin{array}{lllllll}\text { P072_PBO } & 243.259 & 39.521 & -3.655 & 0.470 & 0.400 & 0.400\end{array}$ P615_PBO $243.237 \quad 35.205-6.5095 .371 \quad 1.100 \quad 1.100$ $\begin{array}{llllllll}\mathrm{P} 479 & \mathrm{PBO} & 243.217 & 33.493 & -23.769 & 22.971 & 0.800 & 0.500\end{array}$ $\begin{array}{lllllll}\text { ORMT_PBO } & 243.185 & 34.675 & -11.528 & 10.872 & 0.200 & 0.200\end{array}$ $\begin{array}{lllllll}\text { P606_PBO } & 243.120 & 34.462 & -12.235 & 11.773 & 0.500 & 0.500\end{array}$ $\begin{array}{llllllll}\text { BBRY_PBO } & 243.116 & 34.264 & -12.942 & 14.774 & 0.300 & 0.300\end{array}$ $\begin{array}{lllllll}\text { GOLD_PBO } & 243.111 & 35.425 & -6.511 & 6.274 & 0.246 & 0.259\end{array}$ WOMT_PBO $243.068 \quad 34.669-11.928 \quad 11.875 \quad 0.200 \quad 0.200$ $\begin{array}{lllllll}\text { LNMT_PBO } & 243.060 & 35.090 & -7.614 & 9.275 & 0.200 & 0.200\end{array}$ $\begin{array}{lllllll}\text { P473_PBO } & 243.050 & 32.734 & -29.096 & 27.375 & 0.300 & 0.300\end{array}$ $\begin{array}{llllllll}\text { P584_PBO } & 243.048 & 33.893 & -17.756 & 19.475 & 0.300 & 0.300\end{array}$ BSRY_PBO $242.988 \quad 34.919 \quad-10.020 \quad 11.076 \quad 0.200 \quad 0.200$ $\begin{array}{lllllll}\text { TOIY_PBO } & 242.951 & 39.542 & -3.955 & 1.277 & 0.300 & 0.200\end{array}$ $\begin{array}{lllllll}\text { BILL_PBO } 242.935 & 33.578 & -25.467 & 25.678 & 0.200 & 0.300\end{array}$ $\begin{array}{lllllll}\text { P478_PBO } & 242.928 & 33.236 & -27.279 & 26.878 & 0.200 & 0.900\end{array}$ $\begin{array}{llllllll}\text { P472_PBO } & 242.895 & 32.889 & -28.691 & 27.179 & 0.300 & 0.300\end{array}$ $\begin{array}{lllllll}\text { P589_PBO } & 242.890 & 34.621 & -13.131 & 13.479 & 0.300 & 0.300\end{array}$ $\begin{array}{llllllll}\text { P477_PBO } 242.887 & 33.503 & -24.970 & 24.879 & 0.400 & 0.400\end{array}$ $\begin{array}{llllllll}\text { PPBF_PBO } & 242.818 & 33.836 & -22.655 & 24.236 & 0.574 & 0.557\end{array}$ $\begin{array}{lllllllll}\text { P002_PBO } & 242.813 & 39.521 & -3.557 & 0.281 & 1.700 & 1.700\end{array}$ $\begin{array}{llllllll}\text { P476_PBO } 242.810 & 33.440 & -27.273 & 27.281 & 0.400 & 0.400\end{array}$ $\begin{array}{llllllll}\text { BAMO_PBO } & 242.795 & 40.413 & -3.725 & 1.981 & 0.300 & 0.300\end{array}$ $\begin{array}{lllllll}\text { P } 474 \_ \text {PBO } & 242.751 & 33.355 & -27.606 & 27.439 & 0.626 & 0.654\end{array}$ $\begin{array}{lllllll}\text { P588_PBO } & 242.732 & 34.785 & -10.026 & 11.882 & 0.600 & 0.400\end{array}$ $\begin{array}{lllllll}\text { P586_PBO } & 242.719 & 34.535 & -13.834 & 13.383 & 0.700 & 0.500\end{array}$ $\begin{array}{llllllll}\text { P612_PBO } & 242.684 & 34.187 & -18.247 & 17.384 & 0.500 & 0.800\end{array}$ $\begin{array}{lllllll}\text { MLFP_PBO } & 242.682 & 33.918 & -23.456 & 24.484 & 0.200 & 0.200\end{array}$ P577_PBO 242.681 34.305 $-16.743 \quad 16.384 \quad 0.300 \quad 0.400$ $\begin{array}{lllllll}\text { SCIA_PBO } & 242.612 & 34.607 & -14.391 & 15.547 & 0.552 & 0.557\end{array}$ $\begin{array}{lllllll}\text { P594_PBO } 242.610 & 35.897 & -8.487 & 7.385 & 0.300 & 0.400\end{array}$ $\begin{array}{lllllll}\text { P470_PBO } 242.606 & 34.462 & -15.158 & 14.848 & 0.682 & 0.687\end{array}$ P071_PBO $242.599 \quad 39.347 \quad-2.364 \quad 0.485 \quad 1.700 \quad 1.800$ P595_PBO $242.597 \quad 35.698 \quad-8.194 \quad 7.686 \quad 0.600 \quad 0.600$ 
$\begin{array}{lllllllll}\mathrm{P} 464 & \mathrm{PBO} & 242.590 & 36.159 & -7.078 & 4.886 & 1.200 & 1.300\end{array}$

$\begin{array}{llllllll}\text { ECFS_PBO } 242.588 & 33.648 & -25.666 & 26.886 & 0.200 & 0.200\end{array}$

$\begin{array}{llllllll}\text { MAT2_PBO } 242.563 & 33.857 & -24.054 & 25.669 & 0.505 & 0.512\end{array}$

$\begin{array}{llllllll}\text { EWPP_PBO } 242.474 & 34.104 & -23.951 & 22.688 & 0.200 & 0.200\end{array}$

$\begin{array}{lllllllll}\text { P 4 71_PBO } 242.459 & 33.562 & -26.470 & 26.289 & 0.500 & 0.600\end{array}$

$\begin{array}{lllllll}\text { P583_PBO 242.457 } 34.987 & -9.820 & 12.189 & 0.400 & 0.400\end{array}$

CPBN_PBO 242.427 35.072 $-9.417 \quad 11.889 \quad 0.500 \quad 0.500$

$\begin{array}{llllllll}\text { P069_PBO } & 242.395 & 39.288 & -1.267 & 0.890 & 1.700 & 1.700\end{array}$

$\begin{array}{llllllll}C N P P & -P B O & 242.391 & 33.858 & -24.859 & 25.790 & 0.200 & 0.200\end{array}$

$\begin{array}{llllllll}\text { SBCC_PBO } 242.339 & 33.553 & -27.570 & 28.391 & 0.200 & 0.200\end{array}$

$\begin{array}{lllllll}\text { CCCC_PBO } & 242.329 & 35.565 & -9.500 & 10.492 & 0.200 & 0.200\end{array}$

RAMT_PBO $242.317 \quad 35.339 \quad-8.608 \quad 11.892 \quad 0.200 \quad 0.200$

$\begin{array}{llllllll}\text { PHLB_PBO } 242.306 & 34.925 & -11.264 & 14.707 & 0.587 & 0.592\end{array}$

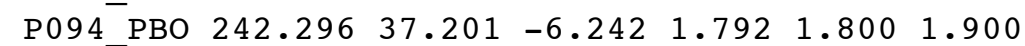

$\begin{array}{lllllll}\text { P581_PBO } 242.271 & 34.510 & -15.637 & 16.293 & 0.500 & 0.500\end{array}$

$\begin{array}{llllllll}\text { LORS_PBO } & 242.246 & 34.133 & -24.824 & 23.004 & 0.570 & 0.574\end{array}$ $\begin{array}{llllllll}\text { HOLM_PBO } & 242.239 & 70.736 & 1.367 & 0.594 & 0.700 & 0.700\end{array}$

$\begin{array}{llllllll}\text { CHMS_PBO } & 242.172 & 34.640 & -14.233 & 15.395 & 0.200 & 0.300\end{array}$

$\begin{array}{llllllll}\text { SPMS_PBO } & 242.151 & 33.993 & -25.589 & 25.572 & 0.545 & 0.550\end{array}$

$\begin{array}{llllllll}A Z U 1 & \end{array}$

$\begin{array}{llllllll}\text { PBPP_PBO } & 242.077 & 34.508 & -16.703 & 18.590 & 0.570 & 0.619\end{array}$

$\begin{array}{lllllllll}\text { CGDM_PBO } & 242.035 & 34.244 & -24.847 & 22.398 & 0.600 & 1.100\end{array}$

$\begin{array}{llllllll}\text { P068_PBO } 242.015 & 39.306 & -3.168 & 0.499 & 1.700 & 1.800\end{array}$

P579_PBO $241.994 \quad 35.039-10.020 \quad 12.999 \quad 1.200 \quad 1.200$

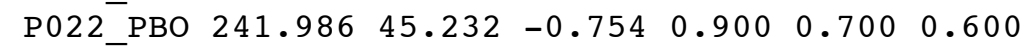

$\begin{array}{llllllll}\mathrm{P} 591 \text {-PBO } 241.984 & 35.152 & -10.116 & 12.600 & 0.500 & 0.400\end{array}$

$\begin{array}{llllllll}\text { RHCL } & \text { PBO } 241.974 & 34.019 & -27.755 & 25.100 & 0.300 & 0.600\end{array}$

BEPK_PBO 241.926 35.878 $-9.990 \quad 11.5010 .300 \quad 0.600$

$\begin{array}{lllllll}\text { BKMS_PBO } 241.905 & 33.962 & -28.858 & 30.001 & 0.300 & 0.500\end{array}$ P468_PBO 241.882 36.976 -8.152 5.202 $1.600 \quad 1.700$

$\begin{array}{llllllll}\text { P099_PBO } 241.841 & 39.212 & -3.772 & 2.503 & 1.500 & 1.600\end{array}$

$\begin{array}{llllllll}\text { BTDM_PBO } 241.812 & 34.293 & -25.647 & 23.304 & 0.300 & 0.300\end{array}$

$\begin{array}{llllllll}\text { P562_PBO } 241.811 & 34.982 & -11.115 & 15.289 & 0.492 & 0.498\end{array}$

$\begin{array}{llllllll}\text { RSTP_PBO } & 241.807 & 34.875 & -12.826 & 15.804 & 0.200 & 0.200\end{array}$

$\begin{array}{llllllll}\text { VDCY_PBO } & 241.780 & 34.179 & -26.451 & 24.204 & 0.300 & 0.200\end{array}$

$\begin{array}{llllllll}\text { PKRD_PBO } 241.767 & 34.072 & -26.954 & 24.505 & 0.300 & 0.300\end{array}$

$\begin{array}{llllllll}\text { CRHS_PBO } & 241.727 & 33.824 & -27.564 & 28.705 & 0.574 & 0.600\end{array}$

$\begin{array}{llllllll}\text { LASC_PBO } & 241.693 & 33.928 & -27.628 & 27.356 & 0.709 & 0.712\end{array}$

$\begin{array}{lllllllll}\text { PVRS_PBO } & 241.679 & 33.774 & -28.881 & 29.721 & 0.587 & 0.596\end{array}$

$\begin{array}{llllllll}\mathrm{ECCO} P \mathrm{PBO} & 241.671 & 33.887 & -27.654 & 29.122 & 0.589 & 0.596\end{array}$

$\begin{array}{llllllll}\text { CAT2_PBO } 241.666 & 33.312 & -29.881 & 32.007 & 0.200 & 0.200\end{array}$

$\begin{array}{llllllll}\text { DSHS_PBO } 241.651 & 34.024 & -27.257 & 26.607 & 0.400 & 0.400\end{array}$

P627_PBO 241.621 37.973 $-4.717 \quad 5.908 \quad 1.700 \quad 1.700$

$\begin{array}{llllllll}\text { P651_PBO } 241.613 & 37.563 & -5.932 & 6.108 & 1.700 & 1.800\end{array}$

$\begin{array}{llllllll}\text { LFRS_PBO } & 241.587 & 34.095 & -28.377 & 26.224 & 0.566 & 0.572\end{array}$

$\begin{array}{llllllll}\text { THCP_PBO } & 241.585 & 35.158 & -12.517 & 13.109 & 0.200 & 0.200\end{array}$

P133_PBO 241.540 38.725 -3.591 4.210 $1.600 \quad 1.600$

$\begin{array}{llllllll}\text { P653_PBO } 241.528 & 37.737 & -3.826 & 6.310 & 1.700 & 1.800\end{array}$

$\begin{array}{llllllll}\text { ISLK_PBO } 241.526 & 35.662 & -11.786 & 10.431 & 0.594 & 0.579\end{array}$

$\begin{array}{llllllll}\mathrm{CAT}_{1} \text { PBO } & 241.517 & 33.446 & -29.873 & 32.636 & 0.520 & 0.550\end{array}$

$\begin{array}{lllllllll}\text { VNCX_PBO } 241.515 & 34.293 & -26.401 & 24.246 & 0.561 & 0.566\end{array}$ $\begin{array}{llllllll}\text { VIMT_PBO } 241.486 & 34.126 & -27.254 & 28.011 & 0.300 & 0.300\end{array}$ $\begin{array}{llllllll}\text { P560_PBO } 241.459 & 34.822 & -16.529 & 17.812 & 0.400 & 0.500\end{array}$ $\begin{array}{llllllll}\text { P556_PBO } 241.455 & 34.771 & -15.431 & 15.712 & 0.700 & 0.800\end{array}$ $\begin{array}{llllllll}\text { P650_PBO } 241.445 & 37.891 & -5.421 & 6.612 & 1.700 & 1.800\end{array}$ $\begin{array}{lllllll}\text { P020_PBO 241.434 47.002 } & 0.108 & 0.812 & 0.200 & 0.300\end{array}$ $\begin{array}{llllllll}\text { LAPC_PBO } & 241.425 & 34.182 & -27.367 & 28.474 & 0.577 & 0.581\end{array}$ $\begin{array}{llllllll}\text { P558_PBO } 241.388 & 35.139 & -13.519 & 12.213 & 1.200 & 1.200\end{array}$ $\begin{array}{lllllllll}\text { CTDM_PBO } & 241.387 & 34.517 & -25.611 & 21.425 & 0.606 & 0.610\end{array}$ P559_PBO $241.382 \quad 34.839 \quad-15.629 \quad 16.113 \quad 0.600 \quad 0.600$ 
$\begin{array}{llllllll}\text { CBHS_PBO } & 241.370 & 34.139 & -28.238 & 29.232 & 0.561 & 0.566\end{array}$

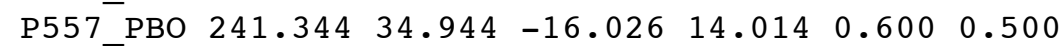
$\begin{array}{llllllll}\text { P643_PBO } 241.302 & 37.562 & -2.233 & 7.815 & 1.600 & 1.700\end{array}$ $\begin{array}{llllllll}\mathrm{P} 021-\mathrm{PBO} & 241.270 & 48.675 & 0.568 & -0.084 & 0.500 & 0.800\end{array}$ $\begin{array}{llllllll}\mathrm{P} 649-\mathrm{PBO} & 241.264 & 37.903 & -5.421 & 7.216 & 1.800 & 1.800\end{array}$ $\begin{array}{llllllll}\text { P453_PBO } 241.255 & 47.759 & 0.534 & 0.216 & 0.600 & 0.500\end{array}$ P567_PBO 241.246 35.421 $-12.609 \quad 10.017 \quad 0.400 \quad 0.400$ $\begin{array}{lllllllll}\text { SFDM_PBO } & 241.245 & 34.460 & -26.543 & 23.517 & 0.300 & 0.400\end{array}$ P571_PBO 241.233 36.231 $-10.681 \quad 8.517 \quad 0.500 \quad 0.900$ $\begin{array}{llllllll}\mathrm{P} 642-\mathrm{PBO} & 241.183 & 37.591 & -13.033 & 2.218 & 1.600 & 1.600\end{array}$ $\begin{array}{llllllll}\text { P646_PBO } 241.180 & 37.677 & -5.230 & 7.518 & 1.600 & 1.700\end{array}$ $\begin{array}{llllllll}\text { EDPP_PBO } & 241.170 & 34.946 & -17.326 & 16.818 & 0.200 & 0.300\end{array}$ $\begin{array}{llllllll}\text { TOST_PBO } & 241.163 & 34.248 & -28.469 & 30.023 & 0.592 & 0.618\end{array}$ $\begin{array}{lllllll}\mathrm{P} 554 \mathrm{PBO} & 241.152 & 34.792 & -19.632 & 19.319 & 1.400 & 1.400\end{array}$ $\begin{array}{llllllll}\text { P639_PBO } 241.131 & 37.655 & -6.631 & 7.219 & 1.700 & 1.700\end{array}$ $\begin{array}{llllllll}\text { MPWD_PBO } & 241.122 & 34.296 & -28.916 & 29.444 & 0.594 & 0.598\end{array}$ $\begin{array}{llllllll}\mathrm{P} 553 \mathrm{PBO} & 241.121 & 34.835 & -19.730 & 17.319 & 0.400 & 0.500\end{array}$ $\begin{array}{llllllll}\text { FMVT_PBO } 241.116 & 34.356 & -28.247 & 28.820 & 0.300 & 0.300\end{array}$ P134_PBO 241.070 38.981 $\quad-5.384 \quad 4.021 \quad 1.600 \quad 1.600$ $\begin{array}{llllllll}\text { CIRX_PBO } 241.063 & 34.110 & -29.156 & 30.321 & 0.300 & 0.200\end{array}$ $\begin{array}{llllllll}\text { P130_PBO } 241.062 & 39.268 & -4.174 & 4.021 & 1.600 & 1.700\end{array}$ $\begin{array}{llllllll}\text { WGPP_PBO } 241.016 & 35.011 & -17.225 & 16.522 & 0.200 & 0.300\end{array}$ $\begin{array}{llllllll}\mathrm{P} 454 \text { PBO } 241.007 & 47.954 & 2.540 & -1.278 & 1.400 & 1.500\end{array}$ P630_PBO 241.000 37.613 -6.433 8.422 $1.600 \quad 1.600$ $\begin{array}{llllllll}\text { KBRC_PBO } 240.992 & 34.399 & -28.046 & 26.022 & 1.300 & 1.400\end{array}$ P648_PBO 240.981 37.800 $-9.826 \quad 8.623 \quad 1.700 \quad 1.800$ $\begin{array}{lllllll}\text { BAR1_PBO } 240.970 & 33.480 & -30.978 & 33.823 & 0.200 & 0.300\end{array}$ $\begin{array}{llllllll}\text { CSCI_PBO } 240.961 & 34.168 & -29.354 & 30.923 & 0.300 & 0.200\end{array}$

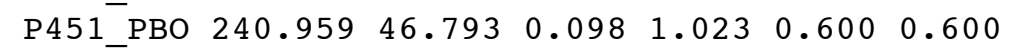
P056_PBO $240.937 \quad 36.027 \quad-13.189 \quad 5.724 \quad 0.500 \quad 1.100$ $\begin{array}{llllllll}\text { SOMT_PBO } & 240.936 & 34.320 & -29.249 & 30.324 & 0.300 & 0.300\end{array}$ $\begin{array}{llllllll}\mathrm{P} 128-\mathrm{PBO} & 240.931 & 39.486 & -4.367 & 3.424 & 1.700 & 1.700\end{array}$ P632_PBO 240.914 37.786 $-11.527 \quad 8.624 \quad 1.700 \quad 1.700$ $\begin{array}{llllllll}\text { NHRG_PBO } & 240.859 & 34.499 & -27.543 & 23.625 & 0.300 & 0.300\end{array}$ $\begin{array}{llllllll}\text { OVLS_PBO } & 240.858 & 34.327 & -28.889 & 29.202 & 0.656 & 0.640\end{array}$ P551_PBO $240.845 \quad 34.856 \quad-22.431 \quad 17.626 \quad 1.800 \quad 2.000$ RSVY_PBO $240.816 \quad 34.541 \quad-28.342 \quad 24.126 \quad 0.300 \quad 0.500$ $\begin{array}{llllllll}\text { HVYS_PBO } & 240.812 & 34.441 & -28.446 & 25.027 & 0.300 & 0.300\end{array}$ P566_PBO 240.771 36.324 $-11.980 \quad 8.127 \quad 0.400 \quad 0.600$ $\begin{array}{llllllll}\mathrm{P} 565 & \end{array}$ $\begin{array}{lllllll}\text { VNCO_PBO } & 240.762 & 34.276 & -31.052 & 29.428 & 0.200 & 0.500\end{array}$ $\begin{array}{llllllll}\mathrm{P} 549-\mathrm{PBO} & 240.674 & 34.600 & -29.241 & 21.430 & 1.700 & 1.700\end{array}$ $\begin{array}{lllllllll}\text { BVPP_PBO } 240.652 & 35.157 & -18.321 & 14.630 & 0.200 & 0.200\end{array}$ $\begin{array}{llllllll}\text { OZST_PBO } 240.647 & 34.683 & -28.787 & 24.343 & 0.644 & 0.634\end{array}$ $\begin{array}{llllllll}A N A 1+P B O & 240.637 & 34.015 & -30.861 & 35.831 & 0.400 & 1.200\end{array}$ $\begin{array}{llllllll}\text { CSST_PBO } & 240.629 & 34.408 & -29.647 & 27.031 & 0.200 & 0.300\end{array}$ $\begin{array}{llllllll}\mathrm{P} 563 & \text { PBO } 240.579 & 35.419 & -13.812 & 12.132 & 0.700 & 0.600\end{array}$

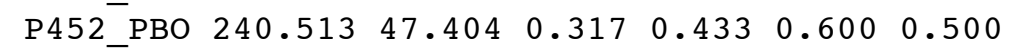
P095_PBO 240.463 39.698 $-6.561 \quad 4.334 \quad 0.800 \quad 0.600$ P450_PBO $240.456 \quad 45.953 \quad 0.364 \quad 1.535 \quad 0.600 \quad 0.400$ P127_PBO 240.400 39.499 $\quad-7.369 \quad 4.336 \quad 0.600 \quad 0.600$ P145_PBO 240.376 41.358 $-4.002 \quad 3.036 \quad 0.600 \quad 0.600$

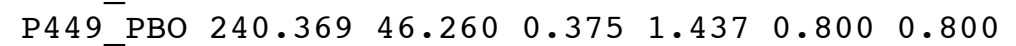
$\begin{array}{llllllll}\mathrm{P} 543-\mathrm{PBO} & 240.287 & 35.319 & -16.017 & 17.038 & 1.200 & 1.300\end{array}$ $\begin{array}{llllllll}\mathrm{RCA} 2-\mathrm{PBO} & 240.280 & 34.500 & -31.831 & 30.441 & 0.656 & 0.642\end{array}$ P139_PBO 240.278 39.908 $-4.855 \quad 4.839 \quad 1.600 \quad 1.700$ $\begin{array}{llllllll}\mathrm{P} 544-\mathrm{PBO} & 240.262 & 35.731 & -12.103 & 11.239 & 0.500 & 0.600\end{array}$ P725_PBO 240.254 37.089 $-10.655 \quad 8.739 \quad 1.500 \quad 1.600$ $\begin{array}{llllllll}C R U 1-P B O & 240.215 & 34.029 & -30.977 & 35.222 & 0.579 & 0.572\end{array}$ $\begin{array}{llllllll}\text { COPR_PBO } 240.120 & 34.415 & -30.649 & 29.242 & 0.300 & 0.200\end{array}$ 
$\begin{array}{llllllll}\text { P5 } 47 & \text { PBO } 240.091 & 35.935 & -9.796 & 11.443 & 0.500 & 0.600\end{array}$

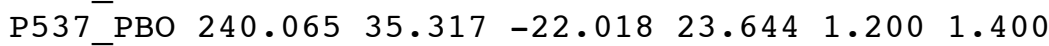
P017_PBO $240.064 \quad 41.276-1.907 \quad 2.944 \quad 2.000 \quad 2.000$ BBDM_PBO $240.018 \quad 34.582 \quad-32.544 \quad 32.745 \quad 0.900 \quad 0.500$ P541_PBO 239.999 $35.687 \quad-13.206 \quad 14.645 \quad 0.500 \quad 0.600$ P448_PBO 239.995 $45.911 \quad 0.460 \quad 1.645 \quad 0.600 \quad 0.400$ $\begin{array}{llllllll}\text { FGST_PBO } & 239.991 & 34.733 & -30.539 & 32.045 & 0.300 & 0.200\end{array}$ $\begin{array}{llllllll}\text { P536_PBO } & 239.975 & 35.280 & -24.620 & 26.346 & 1.300 & 1.400\end{array}$ P307_PBO 239.942 36.947 $-12.861 \quad 9.646 \quad 1.100 \quad 0.700$ SRS1_PBO $239.935 \quad 34.004 \quad-31.465 \quad 35.646 \quad 0.200 \quad 0.200$ P535_PBO 239.899 35.235 -26.222 $28.747 \quad 1.900 \quad 1.900$ P538_PBO 239.888 35.534 $\quad-22.011 \quad 23.748 \quad 1.200 \quad 1.200$ $\begin{array}{llllllll}\text { P540_PBO } & 239.869 & 35.801 & -12.402 & 13.548 & 0.700 & 0.800\end{array}$ $\begin{array}{llllllll}\text { TJRN PBO } & 239.867 & 34.483 & -31.648 & 33.648 & 0.200 & 0.300\end{array}$ $\begin{array}{llllllll}\text { P5 } 46 \text { PBO } 239.845 & 35.928 & -9.598 & 13.049 & 0.600 & 0.700\end{array}$ P539_PBO 239.818 35.703 $-15.306 \quad 18.949 \quad 0.400 \quad 0.400$ P305_PBO 239.803 37.352 -11.248 $7.849 \quad 0.500 \quad 0.500$

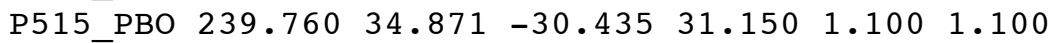
$\begin{array}{lllllllll}\text { P532_PBO } & 239.733 & 35.634 & -22.809 & 27.751 & 0.300 & 0.400\end{array}$ P300_PBO 239.723 36.304 $-14.085 \quad 7.451 \quad 0.300 \quad 0.300$ $\begin{array}{llllllll}\text { ORES_PBO } & 239.721 & 34.739 & -31.504 & 34.268 & 0.598 & 0.624\end{array}$ P298_PBO 239.706 36.016 -9.195 $13.552 \quad 1.600 \quad 1.600$ P310_PBO 239.666 38.736 $-11.199 \quad 8.353 \quad 1.600 \quad 1.700$ P280_PBO 239.652 35.544 $\quad$-24.912 $29.853 \quad 1.200 \quad 1.200$ MIG1_PBO $239.649 \quad 34.038 \quad-31.665 \quad 36.453 \quad 0.300 \quad 0.300$ P529_PBO 239.646 35.440 $\quad-27.016 \quad 30.153 \quad 1.200 \quad 1.200$ P304 PBO 239.643 $36.739 \quad-11.270 \quad 9.053 \quad 0.600 \quad 0.700$ $\begin{array}{llllllll}\text { P296 PBO } 239.636 & 36.052 & -8.994 & 13.653 & 1.500 & 1.500\end{array}$ P533_PBO 239.629 35.748 $-22.805 \quad 29.153 \quad 1.100 \quad 1.100$ P388_PBO 239.622 42.469 $-2.466 \quad 3.354 \quad 1.100 \quad 0.900$ P516_PBO 239.617 35.106 $-30.028 \quad 30.554 \quad 0.600 \quad 0.600$ $\begin{array}{llllllll}\text { P514_PBO } & 239.590 & 35.011 & -29.231 & 32.354 & 1.300 & 1.300\end{array}$ GR8V_PBO $239.584 \quad 36.399 \quad-12.082 \quad 10.554 \quad 0.500 \quad 0.600$ CARH_PBO $239.569 \quad 35.888 \quad-21.993 \quad 28.043 \quad 0.589 \quad 0.762$ P294_PBO 239.560 36.123 $\quad-9.692 \quad 11.055 \quad 1.100 \quad 1.100$ MASW_PBO 239.557 $35.833 \quad-23.602 \quad 28.855 \quad 0.400 \quad 0.300$ LAND_PBO $239.527 \quad 35.900 \quad-22.600 \quad 29.156 \quad 0.500 \quad 0.400$ HOGS_PBO $239.521 \quad 35.867 \quad-23.201 \quad 29.556 \quad 0.400 \quad 0.300$ P530_PBO 239.520 $35.625 \quad-25.910 \quad 31.456 \quad 0.500 \quad 0.800$ $\begin{array}{llllllll}\mathrm{P} 146 \mathrm{PBO} & 239.463 & 39.337 & -10.479 & 7.357 & 1.300 & 1.300\end{array}$ P293_PBO 239.457 $36.089 \quad-8.994 \quad 12.057 \quad 1.400 \quad 1.500$ P528_PBO 239.455 35.328 $-27.42133 .257 \quad 1.400 \quad 1.400$ P297_PBO 239.448 35.974 $\quad-29.398 \quad 34.258 \quad 0.500 \quad 0.600$ $\begin{array}{lllllll}\text { LOWS PBO } & 239.406 & 35.829 & -25.403 & 31.959 & 0.400 & 0.400\end{array}$ P527_PBO 239.395 35.754 $-26.506 \quad 31.659 \quad 1.400 \quad 1.400$ $\begin{array}{llllllll}\text { VNDP_PBO } & 239.384 & 34.556 & -32.538 & 34.830 & 0.355 & 0.359\end{array}$ P302_PBO 239.381 $36.635-12.175 \quad 8.559 \quad 0.500 \quad 0.300$ P306_PBO 239.356 $37.795 \quad-12.434 \quad 7.260 \quad 1.200 \quad 1.300$ USLO_PBO $239.339 \quad 35.312 \quad-28.822 \quad 34.860 \quad 0.400 \quad 0.500$ P445_PBO 239.328 $45.590 \quad 0.245 \quad 2.560 \quad 0.600 \quad 0.500$ P140_PBO 239.307 $38.829 \quad-12.198 \quad 7.061 \quad 1.300 \quad 1.300$ P287_PBO 239.302 36.025 -32.097 31.661 $1.000 \quad 0.400$ P303_PBO 239.295 37.054 $-11.0618 .561 \quad 0.500 \quad 0.400$ $\begin{array}{llllllll}\text { P301_PBO } & 239.257 & 36.806 & -10.870 & 8.362 & 0.500 & 0.300\end{array}$ CRBT_PBO 239.249 35.792 $\quad-27.005 \quad 33.462 \quad 0.400 \quad 0.500$ P148_PBO 239.194 $40.419 \quad-6.342 \quad 3.863 \quad 1.300 \quad 1.400$ P525_PBO 239.192 35.426 $-29.918 \quad 34.063 \quad 1.600 \quad 1.600$ P295_PBO 239.158 35.697 $\quad-27.109 \quad 33.764 \quad 0.300 \quad 0.700$ P526_PBO 239.130 35.636 $\quad-26.911 \quad 35.465 \quad 0.500 \quad 1.300$ P288_PBO 239.121 36.140 $\quad-29.994 \quad 32.065 \quad 0.900 \quad 0.700$ 
$\begin{array}{llllllll}\text { P284_PBO } 239.093 & 35.933 & -29.601 & 31.566 & 0.500 & 0.300\end{array}$ $\begin{array}{llllllll}\text { P065 PBO } & 239.067 & 46.844 & 1.089 & 1.466 & 1.700 & 1.800\end{array}$ $\begin{array}{lllllll}\text { P309_PBO } 239.049 & 38.090 & -9.825 & 7.767 & 0.600 & 0.700\end{array}$ $\begin{array}{lllllll}\text { P285_PBO } 239.019 & 36.417 & -12.585 & 12.467 & 1.200 & 1.200\end{array}$ P067_PBO 238.997 35.552 $\quad-29.415 \quad 34.268 \quad 0.600 \quad 1.700$ $\begin{array}{llllllll}\text { P252 PBO } & 238.942 & 37.170 & -11.358 & 7.869 & 0.500 & 0.600\end{array}$ $\begin{array}{llllllll}\text { P278_PBO } 238.939 & 35.711 & -29.210 & 35.569 & 0.600 & 0.700\end{array}$ P249_PBO 238.936 $36.612 \quad-11.878 \quad 7.569 \quad 1.100 \quad 1.100$ P444_PBO 238.932 $48.730 \quad 0.456 \quad 3.769 \quad 1.200 \quad 1.300$ $\begin{array}{llllllll}\text { P260 PBO } 238.916 & 37.711 & -11.540 & 7.570 & 1.200 & 0.600\end{array}$ P276_PBO $238.905 \quad 38.645 \quad-10.807 \quad 7.070 \quad 0.600 \quad 0.500$ P259_PBO $238.899 \quad 37.433 \quad-12.049 \quad 4.470 \quad 0.900 \quad 0.400$ $\begin{array}{llllllll}\text { P175_PBO } & 238.865 & 36.426 & -28.885 & 32.871 & 1.100 & 1.100\end{array}$ $\begin{array}{lllllll}\text { QCYN PBO } & 238.863 & 36.161 & -29.594 & 35.271 & 1.000 & 0.700\end{array}$ $\begin{array}{lllllll}\text { P247_PBO } & 238.812 & 36.560 & -28.581 & 33.572 & 1.100 & 1.100\end{array}$ P275_PBO $238.785 \quad 38.322 \quad-11.3196 .473 \quad 1.200 \quad 1.300$ P255_PBO 238.675 $37.582 \quad-11.645 \quad 8.275 \quad 1.000 \quad 1.000$ P244_PBO $238.645 \quad 37.011 \quad-13.566 \quad 9.076 \quad 0.500 \quad 0.500$ $\begin{array}{llllllll}\text { P273 PBO } & 238.612 & 38.116 & -10.327 & 5.976 & 0.800 & 0.900\end{array}$ $\begin{array}{llllllll}\text { P2 42_PBO } & 238.537 & 36.954 & -19.068 & 18.578 & 0.700 & 1.000\end{array}$ $\begin{array}{llllllll}\text { P257_PBO } 238.536 & 37.755 & -10.840 & 7.678 & 0.500 & 0.500\end{array}$ HCRO_PBO $238.530 \quad 40.816 \quad-7.069 \quad 5.717 \quad 1.181 \quad 0.843$ P674_PBO $238.510 \quad 41.616 \quad-5.803 \quad 3.379 \quad 1.300 \quad 1.400$ P672_PBO 238.493 $41.712 \quad-3.800 \quad 4.679 \quad 0.800 \quad 1.000$ $\begin{array}{llllllll}\text { P240_PBO } & 238.458 & 37.008 & -20.667 & 22.980 & 0.900 & 0.600\end{array}$ P387_PBO 238.426 44.297 $1.893 \quad 2.081 \quad 1.4001 .500$ $\begin{array}{lllllll}\text { P234 PBO } & 238.409 & 36.859 & -27.972 & 30.181 & 1.200 & 1.200\end{array}$ $\begin{array}{llllllll}\text { P256_PBO } & 238.395 & 37.932 & -11.934 & 8.281 & 0.500 & 0.500\end{array}$ $\begin{array}{llllllll}\mathrm{P} 442-\mathrm{PBO} & 238.384 & 48.260 & 1.336 & 0.682 & 1.400 & 1.200\end{array}$ $\begin{array}{llllllll}\text { P268 PBO } & 238.354 & 38.474 & -14.216 & 7.182 & 1.500 & 0.500\end{array}$ $\begin{array}{lllllll}\text { P217_PBO } 238.349 & 37.104 & -21.464 & 22.082 & 0.500 & 0.700\end{array}$ $\begin{array}{llllllll}\mathrm{P} 432 & \text { PBO } & 238.317 & 46.623 & 1.876 & 2.983 & 0.400 & 0.300\end{array}$ P228_PBO 238.313 $37.602 \quad-12.047 \quad 11.083 \quad 0.500 \quad 0.500$ $\begin{array}{llllllll}\text { P218_PBO } & 238.286 & 37.204 & -21.461 & 22.084 & 1.200 & 0.700\end{array}$ P271_PBO $238.285 \quad 38.657 \quad-11.709 \quad 6.984 \quad 0.600 \quad 0.400$ $\begin{array}{llllllll}\text { P210_PBO } 238.268 & 36.816 & -29.374 & 32.484 & 0.800 & 0.400\end{array}$ P380_PBO 238.220 $42.260 \quad-2.082 \quad 5.485 \quad 0.900 \quad 0.400$ $\begin{array}{lllllll}\text { P227_PBO } & 238.210 & 37.533 & -13.749 & 12.685 & 0.700 & 0.700\end{array}$ $\begin{array}{llllllll}\text { P171_PBO } & 238.207 & 36.486 & -29.786 & 35.986 & 0.300 & 0.800\end{array}$

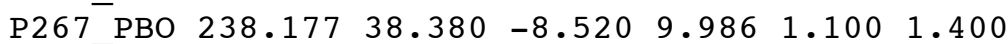
$\begin{array}{lllllll}\text { P226_PBO } & 238.174 & 37.337 & -22.057 & 21.186 & 1.100 & 1.200\end{array}$ $\begin{array}{lllllll}\text { P266_PBO } & 238.156 & 38.184 & -11.627 & 7.587 & 0.800 & 0.700\end{array}$ $\begin{array}{llllllll}\text { P212_PBO } & 238.137 & 36.962 & -27.970 & 30.887 & 1.300 & 1.300\end{array}$ P429_PBO 238.123 45.676 $1.9415 .087 \quad 1.100 \quad 1.200$ $\begin{array}{lllllll}\text { P231_PBO } 238.095 & 36.622 & -30.582 & 35.788 & 1.700 & 1.800\end{array}$ $\begin{array}{llllllll}\text { P272_PBO } & 238.057 & 39.145 & -10.693 & 4.689 & 1.300 & 0.900\end{array}$ $\begin{array}{lllllll}\text { P265_PBO } 238.046 & 38.530 & -9.715 & 7.189 & 1.800 & 0.800\end{array}$

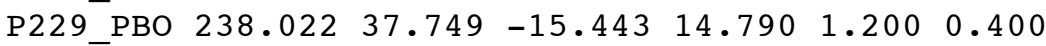
P431_PBO 238.012 $46.572 \quad-0.628 \quad 3.090 \quad 1.4001 .500$

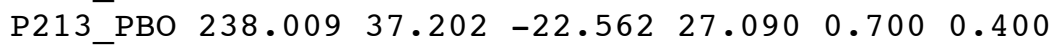
$\begin{array}{llllllll}\text { P344_PBO } 237.972 & 39.929 & -10.566 & 5.991 & 1.400 & 1.400\end{array}$ $\begin{array}{lllllll}\text { P2 } 70-\text { PBO } & 237.945 & 39.244 & -8.791 & 6.591 & 1.000 & 0.900\end{array}$ $\begin{array}{lllllll}\text { P225_PBO } & 237.942 & 37.714 & -15.245 & 17.092 & 0.500 & 0.400\end{array}$ $\begin{array}{lllllll}\text { P222_PBO } & 237.917 & 37.539 & -21.251 & 24.792 & 0.800 & 0.400\end{array}$ $\begin{array}{lllllll}\text { P262_PBO } & 237.904 & 38.025 & -13.534 & 14.492 & 1.300 & 1.100\end{array}$ P701_PBO $237.867 \quad 46.195 \quad-0.742 \quad 2.193 \quad 1.400 \quad 1.600$ WINT_PBO $237.859 \quad 37.653 \quad-19.219 \quad 21.004 \quad 0.558 \quad 0.490$ P696_PBO $237.848 \quad 46.197 \quad 0.858 \quad 3.694 \quad 1.200 \quad 0.900$ P695_PBO $237.836 \quad 46.199 \quad-1.242 \quad 4.894 \quad 1.100 \quad 1.800$ P694_PBO $237.818 \quad 46.300 \quad 2.261 \quad 4.294 \quad 1.400 \quad 1.400$ 


$$
\begin{aligned}
& \begin{array}{lllllll}
\text { P264_PBO } 237.805 & 38.444 & -11.920 & 10.695 & 0.700 & 0.400
\end{array} \\
& \begin{array}{llllllll}
\text { P703 PBO } & 237.804 & 46.145 & 0.855 & 2.895 & 1.500 & 1.600
\end{array} \\
& \text { P693_PBO 237.798 } 46.210 \quad 4.058 \quad 2.395 \quad 1.800 \quad 1.100 \\
& \begin{array}{lllllll}
\text { SLAC_PBO } & 237.796 & 37.417 & -23.956 & 28.495 & 0.400 & 0.300
\end{array} \\
& \text { P655_PBO 237.794 } 41.294 \quad-3.719 \quad 5.495 \quad 1.400 \quad 1.500 \\
& \begin{array}{lllllll}
\text { P261_PBO } & 237.782 & 38.153 & -12.530 & 14.295 & 0.800 & 0.500
\end{array} \\
& \begin{array}{llllllll}
\text { P224_PBO } & 237.781 & 37.864 & -16.240 & 18.795 & 0.700 & 0.700
\end{array} \\
& \text { P691_PBO } 237.773 \quad 46.231 \quad 0.958 \quad 3.295 \quad 1.400 \quad 1.600 \\
& \begin{array}{lllllll}
\text { P345_PBO } 237.729 & 40.271 & -8.255 & 6.196 & 0.900 & 0.600
\end{array} \\
& \begin{array}{llllllll}
\text { P208_PBO } & 237.696 & 39.109 & -10.197 & 6.897 & 0.900 & 1.200
\end{array} \\
& \text { P705_PBO } 237.68946 .173 \quad 1.956 \quad 5.097 \quad 1.400 \quad 1.500 \\
& \text { P349_PBO } 237.681 \quad 40.731 \quad-6.6395 .597 \quad 0.900 \quad 0.600 \\
& \text { P427_PBO 237.659 45.430 } 2.4295 .298 \quad 0.900 \quad 0.700 \\
& \begin{array}{llllllll}
\mathrm{P} 687 & \text { PBO } & 237.645 & 46.110 & 4.553 & 6.398 & 0.600 & 0.500
\end{array} \\
& \begin{array}{llllllll}
\mathrm{P} 689 & \mathrm{PBO} & 237.639 & 46.190 & 4.056 & 4.198 & 0.800 & 0.900
\end{array} \\
& \begin{array}{lllllll}
\mathrm{P} 181 \text {-PBO } & 237.623 & 37.915 & -19.339 & 24.799 & 0.500 & 0.900
\end{array} \\
& \text { P060_PBO } 237.585 \quad 40.998 \quad-5.130 \quad 6.300 \quad 1.000 \quad 0.500
\end{aligned}
$$

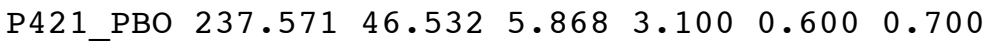

$$
\begin{aligned}
& \begin{array}{lllllll}
\text { P200_PBO } 237.548 & 38.240 & -14.428 & 18.500 & 0.800 & 0.500
\end{array} \\
& \text { P440_PBO } 237.507 \quad 48.856 \quad 1.751 \quad 2.801 \quad 1.000 \quad 1.100 \\
& \begin{array}{llllllll}
\text { P199_PBO } 237.497 & 38.264 & -15.228 & 18.901 & 0.700 & 0.500
\end{array} \\
& \text { P426_PBO } 237.485 \quad 47.803 \quad 1.513 \quad 1.402 \quad 1.300 \quad 1.400 \\
& \text { PCOL_PBO } 237.429 \quad 47.172 \quad 4.490 \quad 6.203 \quad 1.100 \quad 1.100 \\
& \begin{array}{lllllll}
\text { P206_PBO } & 237.424 & 38.778 & -12.910 & 14.203 & 1.400 & 1.300
\end{array} \\
& \text { P379_PBO } 237.423 \quad 44.497 \quad 2.494 \quad 5.703 \quad 1.200 \quad 1.200
\end{aligned}
$$

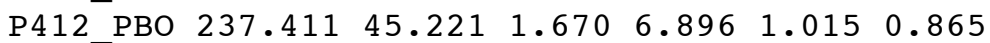

$$
\begin{aligned}
& \begin{array}{llllllll}
\text { P341 } & \text { PBO } & 237.393 & 40.651 & -6.544 & 7.104 & 1.000 & 0.800
\end{array} \\
& \begin{array}{llllllll}
\text { P198_PBO } 237.393 & 38.260 & -17.528 & 22.304 & 0.500 & 0.400
\end{array} \\
& \text { P370_PBO 237.344 42.191 }-1.089 \quad 7.305 \quad 0.900 \quad 1.200 \\
& \text { P438_PBO } 237.330 \quad 48.419 \quad 1.034 \quad 1.105 \quad 1.400 \quad 1.400 \\
& \text { P414_PBO } 237.307 \quad 45.835 \quad 4.541 \quad 4.706 \quad 1.100 \quad 1.100 \\
& \begin{array}{lllllll}
\text { P196_PBO } & 237.257 & 38.298 & -18.028 & 25.207 & 1.200 & 1.100
\end{array} \\
& \text { P197_PBO 237.233 38.429 }-16.123 \quad 26.007 \quad 0.800 \quad 0.700 \\
& \begin{array}{llllllll}
\text { P420_PBO } & 237.134 & 46.589 & 3.967 & 5.510 & 0.700 & 0.800
\end{array} \\
& \text { P377_PBO 237.113 44.052 3.976 5.310 } 1.200 \quad 1.300 \\
& \text { P439_PBO } 237.091 \quad 48.708 \quad 4.143 \quad 3.511 \quad 0.500 \quad 0.500 \\
& \text { TWHL_PBO } 237.077 \quad 47.016 \quad 4.182 \quad 5.811 \quad 0.400 \quad 0.200 \\
& \text { P338_PBO } 237.077 \quad 40.748 \quad-6.942 \quad 6.711 \quad 0.900 \quad 0.800 \\
& \begin{array}{llllllll}
\mathrm{P} 423 & \text { PBO } & 237.059 & 47.288 & 4.692 & 4.811 & 0.400 & 0.400
\end{array} \\
& \begin{array}{llllllll}
\text { P371 PBO } & 236.942 & 43.363 & 0.650 & 6.914 & 1.000 & 0.600
\end{array} \\
& \begin{array}{llllllll}
\mathrm{P} 183 & \text { PBO } & 236.931 & 38.314 & -22.329 & 32.614 & 1.100 & 1.100
\end{array} \\
& \text { P376_PBO 236.898 } 44.941 \quad 2.506 \quad 6.515 \quad 0.300 \quad 0.600 \\
& \begin{array}{lllllll}
\text { P192_PBO } & 236.895 & 39.320 & -15.294 & 14.915 & 0.700 & 0.800
\end{array} \\
& \text { P436_PBO 236.866 } 48.045 \quad 4.618 \quad 3.916 \quad 0.700 \quad 0.900 \\
& \text { P406_PBO 236.848 } 45.190 \quad 3.715 \quad 5.116 \quad 1.100 \quad 1.600 \\
& \text { P411_PBO 236.843 } 45.538 \quad 5.527 \quad 5.016 \quad 1.200 \quad 1.300 \\
& \text { P332_PBO 236.825 } 40.547 \quad-6.851 \quad 7.716 \quad 1.000 \quad 0.700 \\
& \begin{array}{llllllll}
\text { P182_PBO } & 236.819 & 38.495 & -22.323 & 32.617 & 1.500 & 1.600
\end{array} \\
& \begin{array}{llllllll}
\text { P190_PBO } & 236.796 & 39.242 & -17.797 & 18.117 & 0.700 & 0.400
\end{array} \\
& \begin{array}{lllllll}
\text { P188_PBO } & 236.770 & 38.668 & -24.217 & 27.118 & 1.900 & 2.000
\end{array} \\
& \begin{array}{lllllll}
\text { P409_PBO } & 236.761 & 45.851 & 4.938 & 5.518 & 0.600 & 1.400
\end{array} \\
& \begin{array}{lllllll}
\text { P319_PBO } 236.705 & 39.707 & -16.481 & 10.219 & 0.400 & 0.400
\end{array} \\
& \text { P417_PBO 236.702 } 46.575 \quad 4.864 \quad 5.419 \quad 0.500 \quad 0.800 \\
& \text { P373_PBO } 236.667 \quad 43.623 \quad 1.258 \quad 7.120 \quad 0.600 \quad 1.000 \\
& \begin{array}{lllllll}
\text { P189_PBO } & 236.652 & 38.987 & -21.007 & 26.020 & 0.500 & 0.500
\end{array} \\
& \text { P318_PBO 236.628 39.452 }-18.091 \quad 14.421 \quad 1.2001 .300 \\
& \text { P408_PBO 236.623 } 46.201 \quad 5.050 \quad 5.821 \quad 0.600 \quad 0.800 \\
& \begin{array}{llllllll}
\text { P368_PBO } 236.617 & 42.504 & -1.383 & 8.021 & 0.900 & 1.100
\end{array} \\
& \text { P404_PBO } 236.610 \quad 45.159 \quad 4.212 \quad 6.021 \quad 0.900 \quad 1.500 \\
& \text { P418_PBO } 236.592 \quad 47.237 \quad 3.687 \quad 4.022 \quad 1.300 \quad 1.400
\end{aligned}
$$




$$
\begin{aligned}
& \begin{array}{lllllll}
\text { P369 PBO } 236.571 & 43.140 & 0.940 & 8.122 & 0.700 & 0.800
\end{array} \\
& \begin{array}{llllllll}
\mathrm{P} 430 & \text { PBO } & 236.564 & 47.004 & 5.778 & 6.422 & 0.600 & 0.600
\end{array} \\
& \begin{array}{llllllll}
\mathrm{P} 435 \text { PBO } & 236.497 & 48.060 & 6.116 & 4.824 & 0.800 & 1.400
\end{array} \\
& \begin{array}{lllllll}
\text { P317 PBO } & 236.448 & 39.906 & -17.876 & 13.625 & 1.200 & 1.200
\end{array} \\
& \text { P313_PBO 236.435 } 39.554 \quad-21.888 \quad 20.625 \quad 1.200 \quad 1.200 \\
& \begin{array}{lllllll}
\text { P314_PBO } & 236.418 & 39.686 & -22.384 & 19.326 & 0.400 & 0.400
\end{array} \\
& \begin{array}{lllllll}
\text { P374_PBO } & 236.409 & 44.382 & 3.583 & 6.326 & 1.100 & 1.100
\end{array} \\
& \begin{array}{lllllll}
\text { P187_PBO } 236.397 & 39.352 & -21.396 & 23.726 & 0.500 & 1.200
\end{array} \\
& \text { P405_PBO 236.356 } 45.629 \quad 5.828 \quad 6.527 \quad 1.200 \quad 1.300 \\
& \begin{array}{lllllll}
\mathrm{P} 324[\mathrm{PBO} & 236.344 & 40.257 & -11.864 & 13.327 & 1.100 & 1.100
\end{array} \\
& \begin{array}{llllllll}
\text { P164_PBO } & 236.307 & 40.119 & -14.323 & 15.034 & 0.505 & 0.492
\end{array} \\
& \begin{array}{lllllll}
\text { P312_PBO } & 236.302 & 39.529 & -21.490 & 23.428 & 1.200 & 1.200
\end{array} \\
& \text { P326_PBO } 236.301 \quad 40.575 \quad-5.393 \quad 7.992 \quad 0.654 \quad 0.640
\end{aligned}
$$

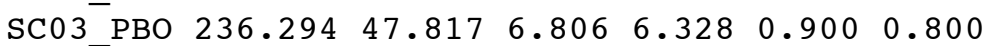

$$
\begin{aligned}
& \begin{array}{lllllll}
\text { P315 PBO } & 236.283 & 39.864 & -22.178 & 19.929 & 0.500 & 0.600
\end{array} \\
& \begin{array}{lllllll}
\text { P059_PBO } & 236.274 & 38.928 & -24.811 & 37.229 & 1.500 & 1.500
\end{array} \\
& \begin{array}{lllllll}
\text { P415_PBO } & 236.270 & 46.656 & 6.864 & 7.729 & 0.500 & 0.500
\end{array} \\
& \text { P165_PBO 236.147 } 40.246 \quad-12.066 \quad 16.432 \quad 1.200 \quad 1.200 \\
& \text { P395_PBO 236.142 45.022 6.504 8.532 } 0.8001 .000 \\
& \begin{array}{lllllll}
\text { P170_PBO } & 236.137 & 40.880 & -0.004 & 13.794 & 1.080 & 0.975
\end{array} \\
& \text { P166_PBO } 236.137 \quad 40.435 \quad-6.559 \quad 15.532 \quad 0.500 \quad 0.800 \\
& \text { P167_PBO 236.120 } 40.544 \quad-4.355 \quad 14.432 \quad 0.900 \quad 0.800 \\
& \begin{array}{llllllll}
\text { P168_PBO } & 236.119 & 40.669 & -1.288 & 13.780 & 0.520 & 0.687
\end{array} \\
& \begin{array}{llllllll}
\text { P325_PBO } & 236.117 & 41.152 & 2.654 & 10.816 & 0.748 & 0.738
\end{array} \\
& \begin{array}{lllllll}
\text { P156_PBO } & 236.094 & 40.024 & -19.174 & 22.233 & 1.200 & 1.300
\end{array} \\
& \begin{array}{lllllll}
\text { P398_PBO } & 236.084 & 46.926 & 7.872 & 8.133 & 1.300 & 1.400
\end{array} \\
& \begin{array}{llllllll}
\mathrm{P} 169 & \mathrm{PBO} & 236.032 & 40.791 & 0.853 & 15.334 & 0.600 & 0.400
\end{array} \\
& \begin{array}{llllllll}
\text { P061 PBO } & 235.986 & 42.967 & 2.030 & 9.235 & 1.100 & 1.400
\end{array} \\
& \begin{array}{lllllll}
\text { P163_PBO } & 235.943 & 40.220 & -14.768 & 23.536 & 1.100 & 1.200
\end{array} \\
& \text { P058_PBO 235.925 } 40.876 \quad 1.355 \quad 17.036 \quad 0.700 \quad 0.700 \\
& \text { P316_PBO 235.914 41.559 } 1.1799 .037 \quad 1.3001 .400 \\
& \begin{array}{llllllll}
\text { P158_PBO } & 235.893 & 40.422 & -5.961 & 22.937 & 0.500 & 0.500
\end{array} \\
& \begin{array}{llllllll}
\text { P160_PBO } & 235.867 & 40.551 & -4.200 & 20.072 & 0.689 & 0.616
\end{array} \\
& \begin{array}{lllllllll}
\text { P403_PBO } 235.859 & 48.062 & 12.111 & 12.938 & 0.800 & 1.100
\end{array} \\
& \text { P161_PBO 235.787 } 40.637 \quad 0.146 \quad 21.740 \quad 0.900 \quad 1.000 \\
& \begin{array}{llllllll}
\text { P162_PBO } & 235.763 & 40.691 & 3.548 & 20.940 & 0.500 & 0.500
\end{array} \\
& \begin{array}{lllllll}
\text { P159_PBO } & 235.717 & 40.505 & -2.059 & 24.741 & 1.500 & 1.600
\end{array} \\
& \text { P157_PBO 235.692 40.248 }-19.168 \quad 31.542 \quad 1.1001 .100 \\
& \begin{array}{llllllll}
\text { P401_PBO } & 235.443 & 47.937 & 15.123 & 10.497 & 0.471 & 0.496
\end{array} \\
& \begin{array}{lllllll}
A I S 1-P B O & 228.400 & 55.069 & -0.203 & 4.198 & 0.800 & 0.500
\end{array} \\
& \text { AB51_PBO } 227.086 \quad 56.798 \quad-2.856 \quad 3.325 \quad 0.700 \quad 0.800 \\
& \text { AB49_PBO } 226.93255 .580 \quad-1.200 \quad 4.228 \quad 1.100 \quad 1.200 \\
& \text { INVK_PBO } 226.473 \quad 68.306 \quad 0.535 \quad 0.937 \quad 0.400 \quad 0.900 \\
& \text { AB50_PBO 225.455 } 58.417 \quad-0.518 \quad 0.958 \quad 1.100 \quad 0.800 \\
& \begin{array}{llllllll}
A B 48 \_P B O & 225.353 & 56.245 & -1.194 & 6.860 & 0.700 & 0.900
\end{array} \\
& \text { AB44_PBO } 224.772 \quad 59.528 \quad 1.313 \quad 4.071 \quad 1.300 \quad 1.100 \\
& \text { BIS1_PBO } 224.461 \quad 56.854 \quad-3.783 \quad 7.878 \quad 0.300 \quad 0.600 \\
& \text { GUS2_PBO } 224.303 \quad 58.418 \quad-0.731 \quad 1.781 \quad 0.400 \quad 0.600 \\
& \text { AB41_PBO } 218.842 \quad 64.777 \quad 1.715 \quad-11.715 \quad 1.500 \quad 1.600 \\
& \begin{array}{lllllll}
A C 65[P B O & 216.296 & 62.832 & -12.486 & 26.731 & 1.800 & 0.800
\end{array} \\
& \text { AB37_PBO 214.548 } 62.967 \quad-26.208 \quad 14.161 \quad 0.500 \quad 0.800 \\
& \text { AC57_PBO 214.257 61.139 }-13.573 \quad 24.266 \quad 1.400 \quad 1.400 \\
& \text { AC63_PBO } 214.153 \quad 63.502 \quad 6.104 \quad-4.032 \quad 0.500 \quad 0.800 \\
& \begin{array}{llllllll}
A C 62-P B O & 213.687 & 63.084 & -22.317 & 6.976 & 0.500 & 0.500
\end{array} \\
& \begin{array}{lllllll}
\text { CLGO_PBO } & 212.140 & 64.874 & 2.418 & -6.499 & 0.500 & 0.700
\end{array} \\
& \begin{array}{llllllll}
\text { PBOC_PBO } & 211.665 & 70.256 & -2.415 & 1.509 & 0.600 & 0.500
\end{array} \\
& \text { GRNR_PBO } 211.022 \quad 63.836 \quad-1.734 \quad-2.080 \quad 0.500 \quad 0.600 \\
& \begin{array}{lllllll}
\text { ATW2_PBO } & 210.868 & 61.598 & -12.709 & 11.022 & 0.700 & 0.800
\end{array} \\
& \text { AC15_PBO 210.276 } 60.481-12.355 \quad 16.931 \quad 1.600 \quad 1.400 \\
& \text { AC53_PBO } 209.93161 .769-5.419 \quad-0.363 \quad 1.5001 .600
\end{aligned}
$$


AB33_PBO 209.827 67.251 $3.157 \quad-8.061 \quad 1.400 \quad 1.500$ AC06_PBO 209.109 59.764 -2.697 4.450 $1.300 \quad 1.200$ SELD_PBO $208.293 \quad 59.446 \quad 1.380 \quad-9.138 \quad 0.800 \quad 0.900$ KOD5_PBO $207.807 \quad 57.618 \quad-9.287 \quad 19.670 \quad 0.500 \quad 0.800$ AC39_PBO 207.606 58.610 3.042 $-8.827 \quad 1.500 \quad 1.700$ AC17_PBO 207.596 60.664 $1.107 \quad-14.527 \quad 1.500 \quad 1.600$ AB28_PBO 207.185 62.094 $1.447 \quad-12.121 \quad 1.200 \quad 1.200$ AC34_PBO 206.721 57.220 $\quad-11.917 \quad 14.186 \quad 1.200 \quad 1.200$ AC59_PBO 206.415 59.567 2.953 -13.310 $0.500 \quad 0.600$ AC27_PBO 205.837 59.253 3.133 -11.301 $0.700 \quad 0.700$ AC45_PBO 205.819 56.564 -14.652 24.799 $1.200 \quad 1.200$ AC02_PBO $205.817 \quad 56.951 \quad-14.140 \quad 14.199 \quad 1.300 \quad 1.700$ AB22_PBO 205.302 59.899 5.145 $-10.994 \quad 1.200 \quad 1.300$ MAUI PBO $203.743 \quad 20.707 \quad-59.690 \quad 53.128 \quad 0.600 \quad 0.700$ SG27 PBO $203.390 \quad 71.323 \quad 0.672 \quad-2.567 \quad 0.500 \quad 1.400$ AB13_PBO 201.496 56.307 -6.332 4.458 $1.300 \quad 1.400$ $\begin{array}{lllllll}A C 21 \_P B O & 200.872 & 55.921 & -8.954 & 4.766 & 1.600 & 1.800\end{array}$ $\begin{array}{lllllll}\text { KOKB_PBO } & 200.335 & 22.126 & -59.844 & 52.241 & 0.632 & 0.592\end{array}$ AB15_PBO 200.122 61.040 2.990 $\quad-6.625 \quad 1.400 \quad 1.600$ $\begin{array}{llllllll}A C 41 \_P B O & 199.593 & 55.909 & -3.977 & 0.882 & 1.300 & 1.400\end{array}$ AB07_PBO $199.52355 .349 \quad-8.395 \quad 4.282 \quad 0.600 \quad 0.800$ AC31_PBO $197.761 \quad 64.638 \quad 3.557 \quad-7.497 \quad 1.200 \quad 1.300$ BAY2_PBO $197.293 \quad 55.190 \quad-6.238 \quad-2.691 \quad 0.500 \quad 1.200$ AB11_PBO $194.627 \quad 64.564 \quad 4.194 \quad-6.162 \quad 1.300 \quad 1.300$ AV15_PBO $194.290 \quad 54.100 \quad-8.923 \quad-4.459 \quad 1.100 \quad 1.600$ AV06_PBO 194.234 54.147 $-3.523-3.558 \quad 1.000 \quad 1.400$ $\begin{array}{llllllll}\mathrm{AV} 14 & \text { PBO } & 194.158 & 54.119 & -2.425 & -5.158 & 1.200 & 1.300\end{array}$ AV12_PBO $194.10254 .211-1.523-1.257 \quad 1.000 \quad 1.800$ AV10_PBO $194.066 \quad 54.098 \quad-3.927 \quad-4.357 \quad 1.400 \quad 1.900$ $\begin{array}{llllllll}A V 08 \text { PBO } & 193.972 & 54.136 & -3.328 & -3.156 & 0.800 & 1.600\end{array}$ AV09_PBO 193.458 53.876 $-6.344 \quad-3.051 \quad 0.900 \quad 1.300$ AB21_PBO $183.337 \quad 51.864 \quad-11.283 \quad 5.330 \quad 1.800 \quad 1.900$ $\begin{array}{llllllll}\text { LLCO_GPS } 243.839 & 31.257 & -32.331 & 31.783 & 0.920 & 0.910\end{array}$ SPMX_GPS $244.534 \quad 31.045 \quad-32.751 \quad 30.337 \quad 1.040 \quad 1.050$ CICE_GPS $243.333 \quad 31.871 \quad-29.954 \quad 30.882 \quad 0.980 \quad 0.990$ PENA_GPS $246.480 \quad 31.354 \quad-2.985 \quad 1.997 \quad 0.830 \quad 0.810$ MAYR_GPS $244.757 \quad 31.988 \quad-25.281 \quad 27.259 \quad 1.210 \quad 1.120$ SCIP_GPS $241.512 \quad 32.914 \quad-31.659 \quad 33.573 \quad 0.970 \quad 0.990$ BLUF_GPS $241.481 \quad 32.927 \quad-30.585 \quad 35.334 \quad 0.880 \quad 0.910$ TWIN_GPS $240.521 \quad 33.232 \quad-31.040 \quad 35.542 \quad 0.890 \quad 0.910$ BRSH_GPS $241.595 \quad 33.407 \quad-29.362 \quad 33.790 \quad 0.860 \quad 0.920$ SBIS_GPS $240.959 \quad 33.472 \quad-31.318 \quad 35.145 \quad 0.930 \quad 0.980$ SNRI_GPS $239.894 \quad 33.951 \quad-30.864 \quad 36.098 \quad 0.940 \quad 0.930$ CENT_GPS $240.247 \quad 33.995 \quad-29.811 \quad 34.414 \quad 0.920 \quad 0.920$ VTIS_GPS $241.706 \quad 33.713 \quad-28.534 \quad 30.755 \quad 1.0201 .050$ PVHS_GPS $241.628 \quad 33.779 \quad-27.745 \quad 29.748 \quad 1.120 \quad 1.140$ TORP_GPS $241.669 \quad 33.798 \quad-28.521 \quad 30.387 \quad 0.910 \quad 0.940$ WRHS_GPS $241.572 \quad 33.958 \quad-27.816 \quad 28.301 \quad 1.160 \quad 1.180$ AOA1_GPS $241.170 \quad 34.157 \quad-29.142 \quad 30.306 \quad 0.860 \quad 0.890$ SPK1_GPS $241.354 \quad 34.059 \quad-28.523 \quad 29.879 \quad 0.870 \quad 0.900$ $\begin{array}{llllllll}\text { UCLP_GPS } & 241.558 & 34.069 & -27.226 & 27.771 & 0.860 & 0.890\end{array}$ OXCO_GPS $241.438 \quad 34.179 \quad-26.753 \quad 30.876 \quad 0.990 \quad 1.000$ MCDS_GPS $241.457 \quad 34.202 \quad-26.457 \quad 30.475 \quad 0.990 \quad 1.000$ $\begin{array}{llllllll}\text { LEEP_GPS } & 241.678 & 34.135 & -27.511 & 25.886 & 0.860 & 0.890\end{array}$ OXYC_GPS $241.793 \quad 34.129 \quad-27.186 \quad 24.512 \quad 1.150 \quad 1.170$ CIT1_GPS $241.873 \quad 34.137 \quad-26.016 \quad 24.639 \quad 0.840 \quad 0.870$ BRAN_GPS $241.723 \quad 34.185 \quad-27.11924 .555 \quad 0.860 \quad 0.890$ 1101 GPS $243.017 \quad 32.569 \quad-28.147 \quad 30.114 \quad 1.050 \quad 1.190$ OTAY_GPS $243.159 \quad 32.601 \quad-26.763 \quad 29.209 \quad 1.480 \quad 1.210$ VA01_GPS $243.442 \quad 32.245 \quad-28.56129 .428 \quad 1.080 \quad 1.100$ 
1102_GPS 243.523 $32.607 \quad-28.04129 .035 \quad 1.030 \quad 1.120$ $\begin{array}{lllllll}\text { LPUR GPS } 244.657 & 32.356 & -19.825 & 27.333 & 0.890 & 0.890\end{array}$ SD17_GPS 242.852 32.818 $\quad-29.209 \quad 29.221 \quad 1.200 \quad 1.500$ SOLJ_GPS $242.747 \quad 32.840 \quad-28.260 \quad 30.155 \quad 1.110 \quad 1.090$ SIO3 GPS $242.750 \quad 32.865 \quad-28.394 \quad 28.615 \quad 0.730 \quad 0.760$ 1106 GPS $243.198 \quad 32.844 \quad-28.021 \quad 28.587 \quad 0.960 \quad 1.040$ SD18_GPS $243.069 \quad 32.912 \quad-28.230 \quad 29.862 \quad 1.030 \quad 1.140$ 1107 GPS 242.723 33.130 $\quad-29.084 \quad 30.186 \quad 1.010 \quad 1.110$ SD21_GPS $243.381 \quad 32.824 \quad-27.29128 .431 \quad 0.950 \quad 1.030$ OCTI_GPS $243.998 \quad 32.734 \quad-28.10128 .357 \quad 0.970 \quad 0.960$ BORD_GPS $244.494 \quad 32.664 \quad-21.202 \quad 26.349 \quad 1.090 \quad 1.070$ T124_GPS $244.500 \quad 32.738 \quad-21.183 \quad 20.719 \quad 0.920 \quad 0.930$ OCOT_GPS $244.204 \quad 32.790 \quad-24.832 \quad 27.120 \quad 0.910 \quad 0.910$ E122 GPS $244.406 \quad 32.800 \quad-17.154 \quad 22.102 \quad 1.320 \quad 1.160$ COLL_GPS $244.498 \quad 32.827 \quad-14.864 \quad 20.255 \quad 0.626 \quad 0.534$ SD35_GPS $243.429 \quad 32.914 \quad-29.609 \quad 28.779 \quad 1.080 \quad 1.180$ MONP GPS $243.578 \quad 32.892 \quad-27.929 \quad 27.943 \quad 0.790 \quad 0.810$ SD15 GPS $243.402 \quad 33.074 \quad-27.797 \quad 27.880 \quad 1.200 \quad 1.740$ L589_GPS $244.239 \quad 32.951 \quad-22.81125 .678 \quad 0.920 \quad 0.890$ ORIE_GPS $244.594 \quad 32.917 \quad-3.299 \quad 4.535 \quad 0.950 \quad 0.910$ HAMA_GPS $244.499 \quad 33.038 \quad-8.675 \quad 2.169 \quad 1.800 \quad 1.720$ CPEI_GPS $245.086 \quad 32.251 \quad-8.913 \quad 7.697 \quad 1.120 \quad 1.130$ MONT_GPS $244.925 \quad 32.559 \quad-6.620 \quad 1.493 \quad 1.100 \quad 1.120$ ASA1_GPS $244.754 \quad 32.629 \quad-8.018 \quad 7.749 \quad 1.050 \quad 0.960$ $\begin{array}{llllllll}\text { JUNC_GPS } & 244.938 & 32.709 & -4.012 & 2.552 & 1.050 & 0.990\end{array}$ GLOC_GPS $244.753 \quad 32.840 \quad-4.513-0.321 \quad 0.920 \quad 0.880$ YUMA GPS $245.797 \quad 32.939 \quad-1.616 \quad 2.961 \quad 1.040 \quad 0.960$ 0301 GPS $242.435 \quad 33.375 \quad-27.443 \quad 29.967 \quad 0.970 \quad 1.020$ 0303_GPS 242.841 33.332 $-26.54529 .301 \quad 1.2601 .440$ PMOB_GPS $243.140 \quad 33.357 \quad-25.496 \quad 27.040 \quad 1.000 \quad 1.020$ SCMS GPS $242.365 \quad 33.444 \quad-28.723 \quad 31.060 \quad 1.020 \quad 1.040$ DANA_GPS $242.291 \quad 33.464 \quad-28.115 \quad 30.922 \quad 0.950 \quad 0.980$ 1202_GPS 242.320 33.469 $-27.775 \quad 29.841 \quad 0.940 \quad 0.960$ KITE_GPS $242.284 \quad 33.554 \quad-28.241 \quad 30.663 \quad 1.080 \quad 0.990$ SJOA_GPS $242.184 \quad 33.602 \quad-28.326 \quad 30.807 \quad 1.240 \quad 1.260$ TRAK_GPS $242.197 \quad 33.618 \quad-26.112 \quad 29.436 \quad 0.850 \quad 0.880$ YUNG_GPS $242.855 \quad 33.430 \quad-27.159 \quad 27.601 \quad 0.980 \quad 1.040$ ROSA_GLA $242.811 \quad 33.505 \quad-25.598 \quad 28.232 \quad 1.160 \quad 1.150$ RDEC_GPS $243.068 \quad 33.468 \quad-24.325 \quad 27.072 \quad 1.160 \quad 1.250$ $\begin{array}{llllllll}A 586 \text { GPS } & 243.164 & 33.484 & -24.013 & 27.239 & 1.080 & 1.140\end{array}$ CARY_GPS $243.265 \quad 33.545 \quad-21.870 \quad 25.815 \quad 1.010 \quad 1.060$ LAKE_GPS $242.645 \quad 33.620 \quad-25.484 \quad 28.329 \quad 1.150 \quad 1.240$ DASH_GPS $242.914 \quad 33.636 \quad-23.507 \quad 27.278 \quad 1.030 \quad 1.070$ R293_GPS 243.152 33.630 $\quad-22.225 \quad 26.429 \quad 1.050 \quad 1.120$ MVFD_GPS $243.475 \quad 33.211 \quad-24.873 \quad 26.527 \quad 1.080 \quad 1.100$ 1108 _GPS $243.307 \quad 33.234-26.733 \quad 27.363 \quad 1.010 \quad 1.100$ 1109 _GPS $243.753 \quad 33.160 \quad-23.474 \quad 24.356 \quad 0.940 \quad 1.030$ SDG6_GPS $243.297 \quad 33.382 \quad-25.094 \quad 27.284 \quad 0.960 \quad 1.000$ 1110 _GPS $244.112 \quad 33.177 \quad-15.159 \quad 16.273 \quad 0.940 \quad 0.970$ EXTR_GPS $244.138 \quad 33.179 \quad-15.889 \quad 14.492 \quad 1.560 \quad 1.510$ COAC_GPS $244.593 \quad 33.196 \quad-3.147 \quad 3.095 \quad 1.050 \quad 0.980$ 1111_GPS 244.481 33.231 $-5.045 \quad 1.399 \quad 1.040 \quad 1.030$ DHLG_GPS $244.212 \quad 33.390 \quad-11.776 \quad 9.899 \quad 0.860 \quad 0.880$ 07NE_GPS $244.248 \quad 33.412 \quad-10.491 \quad 8.198 \quad 1.190 \quad 1.220$ CRAV_GPS 243.299 $33.543 \quad-21.69124 .954 \quad 0.970 \quad 0.990$ HOWY GPS $243.283 \quad 33.550 \quad-21.829 \quad 25.884 \quad 0.980 \quad 1.020$ TBLM_GPS $243.411 \quad 33.527 \quad-20.219 \quad 23.959 \quad 1.010 \quad 1.050$ G114_GPS $243.387 \quad 33.550 \quad-19.132 \quad 23.640 \quad 1.040 \quad 1.080$ LOKT_GPS $243.426 \quad 33.553 \quad-18.982 \quad 22.159 \quad 0.900 \quad 0.930$ ROBO_GPS $243.939 \quad 33.441 \quad-12.295 \quad 16.929 \quad 1.050 \quad 1.050$ 
$\begin{array}{llllllll}\text { M586_GPS } 243.311 & 33.555 & -20.959 & 25.523 & 1.150 & 1.180\end{array}$ ANZA_GLA $243.338 \quad 33.556 \quad-20.779 \quad 24.892 \quad 0.890 \quad 0.910$ G109_GPS 243.366 33.557 $-20.550 \quad 24.921 \quad 1.050 \quad 1.080$ $\begin{array}{lllllll}\text { G120_GPS } 243.397 & 33.565 & -19.758 & 23.490 & 1.160 & 1.200\end{array}$ $\begin{array}{lllllll}\text { RCUT GPS } 243.404 & 33.568 & -18.738 & 22.950 & 1.050 & 1.080\end{array}$ 0821_GPS 243.429 $33.561 \quad-18.770 \quad 20.279 \quad 1.440 \quad 1.670$ G128_GPS 243.442 $33.562 \quad-17.120 \quad 22.638 \quad 1.250 \quad 1.330$ G125_GPS 243.421 33.564 $\quad-20.039 \quad 22.209 \quad 1.240 \quad 1.270$ TOME_GLA $243.320 \quad 33.619 \quad-19.062 \quad 23.293 \quad 0.990 \quad 1.060$ ROCH_GLA $243.390 \quad 33.611 \quad-18.266 \quad 22.650 \quad 0.810 \quad 0.830$ PMCN_GPS $243.483 \quad 33.571 \quad-17.989 \quad 22.267 \quad 1.010 \quad 1.050$ D138_GPS 243.502 33.571 $\quad-16.630 \quad 19.896 \quad 1.170 \quad 1.210$

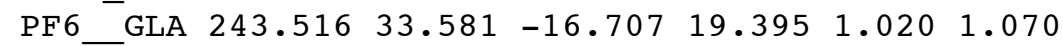
GREN GPS 243.553 $33.574 \quad-16.560 \quad 18.434 \quad 1.120 \quad 1.140$ $\begin{array}{llllllll}\mathrm{PF} 1 & \text { GPS } & 243.570 & 33.584 & -15.618 & 19.483 & 0.960 & 1.000\end{array}$ $\begin{array}{lllllll}\text { PF5C_GPS } 243.523 & 33.603 & -16.332 & 20.605 & 1.180 & 1.150\end{array}$ $\begin{array}{llllllll}\text { PINY GPS } 243.541 & 33.609 & -16.191 & 18.364 & 0.950 & 0.920\end{array}$ $\begin{array}{llllllll}\text { PIN1_GLA } 243.542 & 33.612 & -16.390 & 19.534 & 0.800 & 0.820\end{array}$ $\begin{array}{lllllll}\text { ASBS_GPS } 243.538 & 33.620 & -15.698 & 18.505 & 0.940 & 1.000\end{array}$ BNDY_GPS 243.562 $33.599 \quad-16.764 \quad 21.084 \quad 1.170 \quad 1.180$ T138_GPS 243.580 33.609 $-16.452 \quad 21.363 \quad 1.650 \quad 1.590$ JOES_GPS $243.410 \quad 33.646 \quad-17.397 \quad 20.999 \quad 0.940 \quad 0.950$ L587_GPS $243.589 \quad 33.623 \quad-18.068 \quad 19.863 \quad 1.430 \quad 1.410$ $\begin{array}{llllllll}\text { WHAY_GPS } & 243.528 & 33.684 & -14.991 & 19.665 & 0.970 & 0.970\end{array}$ RSRT_GPS $243.609 \quad 33.688-13.052 \quad 17.582 \quad 0.970 \quad 1.000$ $\begin{array}{llllllll}\text { CAHU_GPS } 243.726 & 33.639 & -12.678 & 15.897 & 1.050 & 1.080\end{array}$ $\begin{array}{llllllll}\text { VORO GPS } & 243.840 & 33.628 & -12.114 & 13.243 & 1.280 & 1.190\end{array}$ TRAN_GPS $244.167 \quad 33.429 \quad-11.445 \quad 9.451 \quad 0.970 \quad 0.980$ S_31_GPS $244.230 \quad 33.427 \quad-8.627 \quad 8.339 \quad 1.300 \quad 1.270$ 25.SE_GPS $244.21733 .441 \quad-10.523 \quad 8.679 \quad 1.070 \quad 1.120$ $\begin{array}{llllllll}\text { SIPH_GPS } 244.322 & 33.427 & -6.999 & 3.485 & 0.920 & 0.940\end{array}$ VARN_GPS $244.086 \quad 33.503 \quad-12.313 \quad 7.904 \quad 1.0601 .020$ PAIN_GPS 243.992 33.612 $\quad-8.992 \quad 7.657 \quad 1.050 \quad 1.070$ N125_GPS $244.138 \quad 33.640 \quad-5.159 \quad 3.792 \quad 1.640 \quad 1.660$ 1113_GPS $244.036 \quad 33.677 \quad-7.076 \quad 4.596 \quad 1.180 \quad 1.380$ $\begin{array}{llllllll}\text { BLAC_GLA } 244.280 & 33.664 & -4.176 & 1.817 & 0.860 & 0.880\end{array}$ $\begin{array}{llllllll}\text { FIFT_GPS } 242.091 & 33.748 & -26.885 & 29.950 & 1.090 & 1.230\end{array}$ SAN1_GLA $242.465 \quad 33.709 \quad-25.575 \quad 26.776 \quad 1.120 \quad 1.100$ $\begin{array}{llllllll}\text { CCCS_GPS } 242.135 & 33.863 & -26.296 & 25.778 & 1.040 & 1.070\end{array}$ $\begin{array}{llllllll}\text { OAKD GPS } 242.402 & 33.847 & -25.267 & 26.788 & 0.920 & 0.960\end{array}$ 0819_GPS 242.453 33.884 $-24.609 \quad 26.506 \quad 0.930 \quad 0.960$ $\begin{array}{llllllll}\text { SJUA_GPS } 242.262 & 33.914 & -25.616 & 26.334 & 0.860 & 0.880\end{array}$ METZ_GLA $242.768 \quad 33.796 \quad-23.020 \quad 25.864 \quad 1.170 \quad 1.120$ $\begin{array}{llllllll}\text { INDO_GLA } & 243.223 & 33.794 & -14.893 & 23.407 & 1.030 & 1.060\end{array}$ $\begin{array}{llllllll}\text { LAST_GLA } & 242.691 & 33.837 & -23.408 & 26.257 & 0.970 & 1.070\end{array}$ $\begin{array}{llllllll}\text { SGHS_GPS } 241.891 & 34.089 & -24.239 & 24.478 & 1.190 & 1.210\end{array}$ SNTZ_GPS 242.116 34.042 $-22.178 \quad 24.849 \quad 1.400 \quad 1.180$ LIMP_GPS $242.451 \quad 33.975 \quad-24.234 \quad 26.006 \quad 1.110 \quad 1.140$ SANO_GPS $242.487 \quad 34.018 \quad-22.734 \quad 24.555 \quad 1.020 \quad 1.050$ JUR3_GPS $242.557 \quad 34.032 \quad-23.122 \quad 24.682 \quad 0.920 \quad 0.930$ $\begin{array}{llllllll}\text { STEE_GPS } 242.470 & 34.077 & -23.348 & 25.685 & 1.170 & 1.200\end{array}$ $\begin{array}{llllllll}\text { PSEB_GPS } 242.311 & 34.121 & -23.962 & 22.612 & 1.070 & 1.110\end{array}$ VERN_GPS 242.274 $34.137 \quad-24.367 \quad 21.493 \quad 1.210 \quad 1.200$ $\begin{array}{llllllll}\text { THRT_GPS } 242.503 & 34.136 & -22.843 & 21.134 & 1.690 & 1.870\end{array}$ $\begin{array}{llllllll}\text { ANGA_GPS } 242.395 & 34.172 & -22.641 & 23.158 & 1.210 & 1.240\end{array}$ SANS_GPS $242.496 \quad 34.212 \quad-20.773 \quad 20.564 \quad 1.080 \quad 1.090$ BRI2_GLA $242.861 \quad 34.014 \quad-17.055 \quad 21.210 \quad 0.940 \quad 0.990$ 0818_GPS 242.896 34.022 $\quad-17.684 \quad 20.569 \quad 1.080 \quad 1.110$ $\begin{array}{llllllll}\text { RTHS_GPS } 242.647 & 34.089 & -22.069 & 22.249 & 0.960 & 0.990\end{array}$ BRYN_GPS $242.734 \quad 34.063 \quad-21.66923 .495 \quad 1.540 \quad 1.750$ 
$\begin{array}{lllllll}\text { CRFP GPS } 242.900 & 34.039 & -18.890 & 19.999 & 0.830 & 0.860\end{array}$ CHER GLA $243.048 \quad 34.003 \quad-14.933 \quad 20.713 \quad 1.090 \quad 1.100$ WD91_GLA $243.288 \quad 33.714 \quad-18.236 \quad 22.384 \quad 0.960 \quad 1.000$ PTHP_GPS $243.701 \quad 33.714 \quad-12.727 \quad 15.788 \quad 0.930 \quad 0.940$ DUNP_GPS 243.719 $33.750 \quad-11.148 \quad 14.008 \quad 1.060 \quad 1.050$ COCH_GLA $243.842 \quad 33.740 \quad-9.384 \quad 9.553 \quad 0.990 \quad 1.020$ $\begin{array}{llllllll}\text { GAPP_GLA } 243.829 & 33.749 & -8.772 & 10.324 & 0.970 & 0.990\end{array}$ BERD_GPS 243.825 33.810 $-7.445 \quad 8.164 \quad 1.050 \quad 1.050$ WIDC_GPS $243.608 \quad 33.935 \quad-10.546 \quad 17.902 \quad 0.930 \quad 0.950$ DESO_GPS $244.600 \quad 33.715 \quad-2.082 \quad 1.145 \quad 1.030 \quad 0.960$ JTRE_GPS $244.236 \quad 33.834 \quad-1.900 \quad 2.868 \quad 1.130 \quad 1.130$ F726_GPS $244.001 \quad 33.974 \quad-4.757 \quad 4.357 \quad 1.570 \quad 1.700$ FORD_GPS $245.011 \quad 33.609 \quad-2.121 \quad 0.930 \quad 1.790 \quad 1.720$ 1114 GPS $244.757 \quad 33.681 \quad-3.575 \quad 1.679 \quad 1.240 \quad 1.060$ BLYT GPS 245.285 $33.610 \quad-2.868 \quad 1.420 \quad 0.800 \quad 0.810$ COXO_GPS $244.773 \quad 34.040 \quad-0.711 \quad 1.039 \quad 1.830 \quad 1.760$ ENDD_GPS $245.519 \quad 34.044 \quad-0.910 \quad 1.221 \quad 1.210 \quad 0.990$ HARV_GPS $239.318 \quad 34.469 \quad-32.739 \quad 34.162 \quad 0.850 \quad 0.900$ LACU_GPS $240.286 \quad 34.494 \quad-30.698 \quad 30.462 \quad 0.960 \quad 0.960$ RUS1_GPS $239.373 \quad 34.571 \quad-29.703 \quad 36.709 \quad 1.320 \quad 1.230$ ALVA_GPS $239.383 \quad 34.593 \quad-31.057 \quad 35.479 \quad 1.350 \quad 1.230$ GAVI_GPS $239.801 \quad 34.502 \quad-29.833 \quad 33.182 \quad 1.030 \quad 0.980$ SNP2_GPS $240.990 \quad 34.440 \quad-29.341 \quad 26.294 \quad 1.260 \quad 1.280$ ROCK_GPS $241.324 \quad 34.236 \quad-27.485 \quad 30.340 \quad 0.880 \quad 0.910$ CHT3_GPS $241.359 \quad 34.257 \quad-27.810 \quad 29.939 \quad 0.840 \quad 0.870$ CSN1_GPS $241.476 \quad 34.254 \quad-27.004 \quad 26.394 \quad 0.900 \quad 0.930$ DELO GPS $241.489 \quad 34.258 \quad-24.993 \quad 26.514 \quad 1.110 \quad 1.160$ OATT GPS $241.399 \quad 34.330 \quad-30.291 \quad 24.067 \quad 1.250 \quad 1.280$ PICO_GNR 241.399 $34.331 \quad-28.02126 .197 \quad 0.810 \quad 0.840$ CMP9_GPS $241.589 \quad 34.353 \quad-26.280 \quad 24.490 \quad 0.870 \quad 0.900$ 0102 _GPS $240.736 \quad 34.566 \quad-29.500 \quad 25.324 \quad 1.290 \quad 1.170$ MUNS_GPS $240.700 \quad 34.636 \quad-27.650 \quad 24.475 \quad 0.970 \quad 0.950$ LVMS_GPS $240.896 \quad 34.734 \quad-24.68921 .027 \quad 1.140 \quad 1.170$ WKPK_GPS $241.259 \quad 34.569 \quad-24.969 \quad 21.394 \quad 0.514 \quad 0.494$ QHTP_GPS $241.755 \quad 34.629 \quad-19.131 \quad 19.353 \quad 1.150 \quad 1.170$ LOSP_GPS $239.394 \quad 34.894 \quad-30.686 \quad 35.288 \quad 0.970 \quad 0.960$ LAMO_GPS $239.743 \quad 34.798 \quad-31.031 \quad 34.814 \quad 1.260 \quad 1.160$ 7HLI_GPS $239.700 \quad 34.960 \quad-29.106 \quad 34.176 \quad 1.530 \quad 1.430$ SALI_GPS $240.28634 .823-28.40929 .722 \quad 0.890 \quad 0.920$ 0504 GPS $240.162 \quad 35.003 \quad-27.296 \quad 28.747 \quad 0.980 \quad 1.000$ LGO7_GPS $240.240 \quad 35.036 \quad-25.799 \quad 25.904 \quad 0.890 \quad 0.920$ MADC_GPS $239.933 \quad 35.076 \quad-27.400 \quad 30.696 \quad 0.940 \quad 0.930$ YAM2_GPS $240.516 \quad 34.852 \quad-25.237 \quad 25.103 \quad 1.040 \quad 0.980$ FZHS_GPS $241.107 \quad 34.800 \quad-20.907 \quad 19.999 \quad 1.010 \quad 1.030$ CUYA_GPS $240.51134 .928 \quad-23.616 \quad 23.373 \quad 1.200 \quad 1.140$ PATW_GPS $240.568 \quad 34.960 \quad-21.149 \quad 21.191 \quad 0.900 \quad 0.910$ J976_GPS $240.821 \quad 35.002 \quad-15.354 \quad 17.630 \quad 1.070 \quad 1.100$ DBL1_GPS $241.513 \quad 35.033-14.194 \quad 14.873 \quad 1.110 \quad 1.200$ WEED_GPS $241.068 \quad 35.223 \quad-12.850 \quad 11.241 \quad 1.160 \quad 1.250$ JACK_GPS $241.340 \quad 35.089 \quad-13.584 \quad 14.700 \quad 1.220 \quad 1.310$ RSPG_GPS $241.330 \quad 35.138 \quad-13.951 \quad 13.440 \quad 0.990 \quad 1.080$ PORT_GPS $241.843 \quad 35.087 \quad-10.619 \quad 14.400 \quad 0.970 \quad 1.000$ SUMT_GPS $241.591 \quad 35.134 \quad-10.199 \quad 13.550 \quad 1.080 \quad 1.210$ PAJA_GPS $241.705 \quad 35.121 \quad-11.336 \quad 14.025 \quad 1.210 \quad 1.320$ 0617 GPS $241.375 \quad 35.274 \quad-12.295 \quad 12.778 \quad 0.960 \quad 1.020$ BLAN_GPS $238.716 \quad 35.665-29.395 \quad 37.577 \quad 1.000 \quad 1.010$ 0509 GPS $238.516 \quad 35.992 \quad-29.539 \quad 36.375 \quad 1.040 \quad 1.030$ BLHL_GPS $239.168 \quad 35.359 \quad-28.812 \quad 36.108 \quad 0.820 \quad 0.860$ TESS_GPS $239.302 \quad 35.386 \quad-27.448 \quad 34.942 \quad 0.920 \quad 0.960$ POZO_GPS $239.701 \quad 35.346 \quad-25.740 \quad 30.556 \quad 1.140 \quad 1.080$ 
$\begin{array}{llllllll}\text { BARR GPS } & 239.427 & 35.456 & -27.432 & 33.707 & 0.870 & 0.910\end{array}$

$\begin{array}{lllllll}\text { ALMO GPS } & 239.547 & 35.552 & -25.419 & 32.082 & 0.840 & 0.870\end{array}$

L623_GPS 239.707 35.583 $-24.155 \quad 29.526 \quad 1.420 \quad 1.030$

GOUD_GPS $240.234 \quad 35.414 \quad-13.755 \quad 16.684 \quad 1.390 \quad 1.190$

POSO_GPS $239.887 \quad 35.520 \quad-20.827 \quad 25.478 \quad 0.950 \quad 0.920$

C616_GPS $239.999 \quad 35.575 \quad-16.365 \quad 19.304 \quad 0.960 \quad 0.920$

H623_GPS 239.654 35.607 $-24.847 \quad 30.578 \quad 1.090 \quad 1.010$

REDH_GPS $239.739 \quad 35.605 \quad-23.409 \quad 27.394 \quad 0.830 \quad 0.860$

P807_GPS $240.146 \quad 35.603 \quad-12.631 \quad 15.518 \quad 0.920 \quad 0.890$

FIBR_GPS $240.606 \quad 35.398 \quad-13.590 \quad 13.059 \quad 0.880 \quad 0.880$

TARO_GPS $239.953 \quad 35.889 \quad-8.686 \quad 16.016 \quad 0.940 \quad 0.930$

0609 GPS $240.712 \quad 36.289-10.836 \quad 11.815 \quad 1.000 \quad 1.020$

CHIL_GPS $241.974 \quad 34.333 \quad-24.406 \quad 22.685 \quad 0.840 \quad 0.880$

CUTT_GPS 242.395 $34.362 \quad-17.320 \quad 22.048 \quad 1.840 \quad 1.600$

CAJO GLA 242.549 $34.347 \quad-17.718 \quad 18.752 \quad 0.910 \quad 0.940$

DVPB_GPS $242.140 \quad 34.413 \quad-22.189 \quad 20.598 \quad 1.110 \quad 1.140$

0705 GLA $242.235 \quad 34.493 \quad-15.780 \quad 18.175 \quad 1.350 \quad 1.050$

MILU_GLA $242.708 \quad 34.281 \quad-17.730 \quad 19.046 \quad 0.950 \quad 0.980$

AVRY_GPS $242.846 \quad 34.468 \quad-14.664 \quad 14.281 \quad 1.010 \quad 1.030$

PT65_GLA 242.932 $34.454 \quad-15.450 \quad 15.068 \quad 1.190 \quad 0.990$

VNPS_GPS $241.879 \quad 34.502 \quad-22.268 \quad 20.548 \quad 1.120 \quad 1.150$

DIP0_GPS $242.569 \quad 34.635 \quad-14.071 \quad 14.932 \quad 1.760 \quad 1.810$

0817_GPS 242.758 $34.537 \quad-14.413 \quad 14.774 \quad 1.000 \quad 1.050$

ITER_GPS $242.770 \quad 34.630 \quad-12.928 \quad 14.004 \quad 1.120 \quad 1.170$

SUNH_GPS $242.703 \quad 34.749 \quad-15.284 \quad 13.086 \quad 1.720 \quad 1.720$

0805_GPS 242.471 35.007 $-8.908 \quad 13.775 \quad 1.960 \quad 1.480$

HODG GPS $242.830 \quad 34.834 \quad-11.715 \quad 13.762 \quad 1.590 \quad 1.730$

SOAP GPS $243.019 \quad 34.904 \quad-10.521 \quad 11.544 \quad 0.890 \quad 0.910$

0809_GPS $244.673 \quad 34.806-1.434 \quad 0.632 \quad 1.550 \quad 1.260$

0803 GPS 243.585 35.072 $-6.181 \quad 2.063 \quad 1.090 \quad 1.140$

NEED_GPS $245.396 \quad 34.807 \quad-2.714 \quad 1.286 \quad 1.110 \quad 0.980$

0806 _GPS $242.386 \quad 35.366 \quad-7.998 \quad 11.529 \quad 1.350 \quad 1.150$

PAOS_GPS 242.294 35.513 $\quad-9.085 \quad 11.262 \quad 1.380 \quad 1.360$

MOJ1_GPS $243.109 \quad 35.332 \quad-5.007 \quad 7.375 \quad 0.395 \quad 0.396$

MOJM_GPS 243.112 35.331 $\quad-5.007 \quad 7.375 \quad 0.395 \quad 0.396$

INYO_GPS 242.188 $35.647 \quad-9.495 \quad 11.416 \quad 0.930 \quad 0.940$

0801 _GPS $244.577 \quad 35.541 \quad-1.892 \quad 1.626 \quad 1.2201 .530$

0915_GPS 243.700 35.867 $-3.537 \quad 2.558 \quad 1.050 \quad 1.090$

0914 GPS $242.671 \quad 35.978 \quad-6.277 \quad 5.778 \quad 0.940 \quad 0.920$

0912 GPS $243.584 \quad 36.304 \quad-2.603 \quad 2.023 \quad 0.930 \quad 1.030$

0607 GPS $239.646 \quad 36.501 \quad-7.627 \quad 10.478 \quad 1.240 \quad 1.050$

0605_GPS $240.882 \quad 36.738 \quad-9.374 \quad 11.598 \quad 1.100 \quad 1.030$

INDE_GPS $241.815 \quad 36.781 \quad-6.608 \quad 8.591 \quad 1.220 \quad 1.010$

MN71_GPS $243.851 \quad 36.994 \quad-1.687 \quad 0.453 \quad 1.040 \quad 1.030$

RICM_GPS $279.616 \quad 25.614 \quad-0.335 \quad 0.412 \quad 0.342 \quad 0.335$

MDO1_GPS $255.985 \quad 30.680 \quad-0.980 \quad 0.153 \quad 0.402 \quad 0.401$

PIE1_GPS $251.881 \quad 34.301-0.892 \quad-0.2210 .405 \quad 0.405$

H217_GPS $237.058 \quad 40.652 \quad-4.948 \quad 6.067 \quad 1.040 \quad 0.810$

0221 _GPS $237.064 \quad 40.370 \quad-6.406 \quad 4.830 \quad 0.870 \quad 0.760$

0108 _GPS $236.507 \quad 40.815-3.8167 .714 \quad 1.000 \quad 0.820$

$01 \mathrm{NE}$ _GPS $236.325 \quad 40.439 \quad-6.259 \quad 13.021 \quad 0.990 \quad 0.620$

01QF_GPS $236.675 \quad 40.815-4.377 \quad 6.038 \quad 1.050 \quad 0.900$

$01 \mathrm{RE}$ GPS $236.377 \quad 40.944 \quad-2.752 \quad 8.703 \quad 0.690 \quad 0.660$

01PA_GPS 235.744 $40.589 \quad 0.515 \quad 21.995 \quad 0.890 \quad 0.850$

1468_GPS 235.844 $40.448 \quad-5.210 \quad 24.761 \quad 1.810 \quad 0.950$

01XD_GPS $236.156 \quad 41.874 \quad 1.971 \quad 8.923 \quad 1.120 \quad 1.020$

BEAR_GPS 235.706 $40.498 \quad-3.857 \quad 27.120 \quad 0.400 \quad 0.360$

COOS_GPS 235.734 $40.257 \quad-18.984 \quad 30.838 \quad 0.520 \quad 0.440$

CVR3_GPS 236.752 39.771 $-13.257 \quad 9.834 \quad 1.110 \quad 0.890$

CW07_GPS 235.905 41.192 4.018 13.181 $0.930 \quad 0.930$ 
GORD_GPS $236.063 \quad 41.986 \quad 0.338 \quad 7.954 \quad 0.770 \quad 0.610$ GRSH GPS $236.022 \quad 40.306 \quad-10.275 \quad 20.077 \quad 0.460 \quad 0.390$ HORS_GPS 236.267 $40.875 \quad-0.903 \quad 8.744 \quad 0.690 \quad 0.600$ H111_GPS 235.967 $41.522 \quad 2.999 \quad 10.611 \quad 1.450 \quad 1.320$ HP12_GPS $236.21141 .993 \quad 2.158 \quad 8.529 \quad 1.650 \quad 1.460$ HP14_GPS $236.799 \quad 39.795 \quad-9.893 \quad 7.393 \quad 0.790 \quad 0.630$ HP15_GPS $236.165 \quad 39.777 \quad-23.242 \quad 24.349 \quad 0.630 \quad 0.530$ HP16_GPS $236.477 \quad 40.460 \quad-6.674 \quad 7.036 \quad 0.710 \quad 0.580$ HP19_GPS $235.883 \quad 40.975 \quad 4.040 \quad 14.577 \quad 0.500 \quad 0.460$ HP21_GPS $236.807 \quad 41.842 \quad 0.291 \quad 6.468 \quad 0.720 \quad 0.600$ KNEE_GPS $236.025 \quad 40.727 \quad-0.297 \quad 14.929 \quad 0.560 \quad 0.480$ KNGP_GPS $235.876 \quad 40.157 \quad-35.502 \quad 46.832 \quad 0.970 \quad 0.960$ L229_GPS 235.623 40.452 $-6.902 \quad 32.104 \quad 1.400 \quad 1.110$ LASS_GPS $236.446 \quad 40.334 \quad-8.982 \quad 8.507 \quad 0.560 \quad 0.490$ P229_GPS 235.628 $40.392 \quad-7.750 \quad 28.597 \quad 0.880 \quad 0.720$ POIR_GPS $236.969 \quad 39.729 \quad-7.697 \quad 6.354 \quad 0.800 \quad 0.640$ SIS3_GPS $235.798 \quad 40.715 \quad 4.230 \quad 19.251 \quad 0.770 \quad 0.670$ TAB3_GPS 235.806 40.630 $0.066 \quad 19.356 \quad 1.230 \quad 1.080$ BARN GPS $288.840 \quad 44.099 \quad 0.484 \quad 0.154 \quad 0.900 \quad 0.800$ USNO_GPS $282.934 \quad 38.919 \quad-0.460 \quad 0.237 \quad 0.500 \quad 0.500$ AMC2_GPS 255.475 $38.803 \quad-1.536 \quad-0.189 \quad 0.600 \quad 0.500$ $\begin{array}{llllllll}\text { PKDB_GPS } 239.458 & 35.945 & -23.488 & 30.760 & 0.500 & 0.500\end{array}$ JAST_GPS $239.279 \quad 38.340-11.357 \quad 7.015 \quad 0.600 \quad 0.600$ 05UH_GPS $239.004 \quad 36.410 \quad-10.644 \quad 13.498 \quad 1.000 \quad 0.700$ ONIE_GPS $238.940 \quad 37.080-11.897 \quad 7.916 \quad 0.500 \quad 0.500$ H104_GPS 238.822 $37.464 \quad-11.326 \quad 7.652 \quad 0.500 \quad 0.500$ 05WG GPS $238.816 \quad 36.571 \quad-29.534 \quad 34.854 \quad 0.900 \quad 0.900$ OSI2_GPS 238.799 $36.294 \quad-27.70135 .660 \quad 1.100 \quad 0.900$ 05WF_GPS $238.730 \quad 36.696-17.318 \quad 19.780 \quad 0.900 \quad 0.900$ PACH_GPS $238.712 \quad 37.008 \quad-14.048 \quad 12.685 \quad 0.600 \quad 0.600$ TURK_GPS $238.701 \quad 36.900-11.875 \quad 9.589 \quad 0.600 \quad 0.600$ 0512_GPS $238.677 \quad 36.420 \quad-26.289 \quad 36.596 \quad 0.800 \quad 0.700$ 05YF_GPS $238.675 \quad 36.793-12.203 \quad 12.496 \quad 0.600 \quad 0.600$ OSR1_GPS $238.625 \quad 37.508-13.046 \quad 8.312 \quad 0.500 \quad 0.600$ 05VE_GPS $238.571 \quad 36.493 \quad-29.288 \quad 34.828 \quad 0.700 \quad 0.600$ RAIL_BVU 238.538 36.909 $-17.296 \quad 20.538 \quad 0.700 \quad 0.700$ MOCH_GPS 238.444 37.477 $\quad-13.980 \quad 9.166 \quad 0.500 \quad 0.500$ MEDA_GPS $238.434 \quad 37.755 \quad-12.518 \quad 8.169 \quad 0.700 \quad 0.800$ 77RT_GPS 238.432 36.873 $-24.62127 .570 \quad 0.600 \quad 0.600$ GAMB_GPS $238.423 \quad 36.055 \quad-25.412 \quad 33.273 \quad 1.800 \quad 1.800$ PGN4_GPS $238.415 \quad 36.887 \quad-23.120 \quad 28.375 \quad 0.600 \quad 0.600$ GILR_GPS $238.384 \quad 36.980 \quad-20.103 \quad 22.584 \quad 0.500 \quad 0.500$ TORO_GPS $238.372 \quad 36.540 \quad-26.406 \quad 32.588 \quad 1.800 \quad 1.800$ BORO_GPS $238.341 \quad 36.722 \quad-30.169 \quad 34.697 \quad 1.600 \quad 0.800$ SALI_GPS $238.334 \quad 36.698 \quad-28.375 \quad 33.499 \quad 1.400 \quad 0.800$ 05YC_GPS $238.332 \quad 36.791 \quad-28.955 \quad 33.099 \quad 0.600 \quad 0.500$ $04 \mathrm{AL} \_$GPS $238.327 \quad 36.914 \quad-26.527 \quad 31.601 \quad 0.700 \quad 0.600$ B112_GPS 238.325 37.694 $-10.850 \quad 8.502 \quad 1.000 \quad 1.100$ USG7_GPS $238.297 \quad 37.622 \quad-12.169 \quad 11.410 \quad 0.800 \quad 0.900$ PERR_GPS 238.294 37.184 $\quad-19.370 \quad 21.111 \quad 1.500 \quad 1.600$ $\begin{array}{llllllll}\text { METC_GPS } 238.286 & 37.229 & -20.961 & 21.613 & 0.600 & 0.500\end{array}$ MINN_GPS $238.278 \quad 37.954 \quad-14.098 \quad 6.016 \quad 1.200 \quad 1.300$ $\begin{array}{llllllll}\text { COY_GPS } & 238.262 & 37.219 & -21.367 & 21.921 & 0.500 & 0.500\end{array}$ 0513_GPS $238.250 \quad 36.763 \quad-28.173 \quad 33.425 \quad 0.600 \quad 0.500$ 05ZB_GPS 238.229 $36.846 \quad-28.557 \quad 32.430 \quad 0.700 \quad 0.600$ FORD_GPS 238.228 $36.589 \quad-26.816 \quad 37.331 \quad 1.200 \quad 1.100$ $\begin{array}{lllllll}\text { CAAA GPS } & 238.217 & 37.186 & -22.082 & 22.634 & 0.500 & 0.500\end{array}$ MAZZ_GPS 238.212 37.137 -22.293 24.536 $0.500 \quad 0.600$ JOBE_GPS 238.208 37.922 $-11.915 \quad 9.237 \quad 0.700 \quad 0.700$ MULL_GPS $238.20136 .749-31.883 \quad 35.5390 .700 \quad 0.700$ 
NDDD_GPS $238.191 \quad 37.069 \quad-22.612 \quad 28.241 \quad 0.500 \quad 0.500$ SARE GPS $238.190 \quad 37.594 \quad-13.493 \quad 11.842 \quad 0.600 \quad 0.600$ FEIF_GPS $238.186 \quad 36.235 \quad-27.805 \quad 36.243 \quad 1.800 \quad 1.700$ CORR_GPS $238.167 \quad 37.012 \quad-25.229 \quad 31.449 \quad 0.500 \quad 0.500$ $\begin{array}{llllllllll}\text { LP } 4 & \text { GPS } & 238.161 & 37.050 & -25.121 & 29.551 & 0.700 & 0.700\end{array}$ LOMA_GPS $238.156 \quad 37.111 \quad-21.707 \quad 26.352 \quad 0.600 \quad 0.600$ MOLR_GPS $238.149 \quad 36.288 \quad-27.798 \quad 34.755 \quad 1.700 \quad 1.700$ SHER_GPS $238.130 \quad 37.541 \quad-14.414 \quad 18.160 \quad 0.600 \quad 0.600$ ALLI_GPS $238.129 \quad 37.499 \quad-15.423 \quad 16.560 \quad 0.600 \quad 0.600$ LEON_GPS $238.126 \quad 36.946 \quad-27.950 \quad 32.961 \quad 0.600 \quad 0.600$ MILS_GPS $238.112 \quad 37.540 \quad-15.817 \quad 16.965 \quad 0.600 \quad 0.600$ $\begin{array}{llllllll}L P 2 & \text { GPS } & 238.091 & 37.104 & -24.219 & 28.672 & 0.600 & 0.600\end{array}$ 1582_GPS 238.081 37.507 $-16.329 \quad 16.874 \quad 1.000 \quad 1.100$ PORT GPS $238.080 \quad 37.004 \quad-26.244 \quad 32.674 \quad 0.500 \quad 0.500$ GREG_GPS $238.076 \quad 36.982 \quad-28.749 \quad 32.476 \quad 0.500 \quad 0.500$ SOBR_GPS $238.071 \quad 36.449 \quad-29.472 \quad 36.677 \quad 1.800 \quad 1.700$ CAIS GPS 238.065 $37.513 \quad-16.630 \quad 17.679 \quad 1.000 \quad 0.800$ FIRE_GPS $238.062 \quad 37.047 \quad-26.537 \quad 31.380 \quad 0.600 \quad 0.600$ SUNS_GPS $238.060 \quad 37.643-16.601 \quad 16.481 \quad 0.600 \quad 0.600$ BURD_GPS $238.053 \quad 37.110 \quad-25.423 \quad 28.183 \quad 0.500 \quad 0.400$ 3814 _GPS $238.048 \quad 37.806 \quad-13.866 \quad 12.785 \quad 1.000 \quad 1.000$ WINE_1PS 238.048 $37.532 \quad-14.528 \quad 16.485 \quad 1.400 \quad 1.400$ BRUC_GPS 238.039 37.073 $\quad-25.034 \quad 31.987 \quad 0.500 \quad 0.500$ VASO_GPS $238.034 \quad 37.247 \quad-20.895 \quad 24.689 \quad 0.800 \quad 0.800$ ODAM_GPS $238.027 \quad 37.180 \quad-22.312 \quad 26.290 \quad 1.100 \quad 1.100$ Z137_GPS 238.026 37.480 $-19.843 \quad 23.090 \quad 0.600 \quad 0.700$ SNJO GPS $238.023 \quad 37.206 \quad-21.606 \quad 26.391 \quad 0.500 \quad 0.500$ TRAL_GPS 238.006 $37.059 \quad-26.242 \quad 33.497 \quad 0.500 \quad 0.500$ CAML_GPS $238.005 \quad 38.417-8.1359 .097 \quad 0.600 \quad 0.600$ WED2_GPS 238.005 37.738 $-14.988 \quad 16.397 \quad 0.600 \quad 0.600$ BRIG BVU $238.004 \quad 37.185 \quad-22.214 \quad 27.597 \quad 0.400 \quad 0.400$ AWIS_GPS 237.998 37.593 $-16.122 \quad 16.099 \quad 1.200 \quad 1.100$ GARS_GPS $237.989 \quad 37.645 \quad-17.511 \quad 17.202 \quad 0.600 \quad 0.600$ ELSE_GPS $237.977 \quad 37.217 \quad-22.210 \quad 27.705 \quad 0.500 \quad 0.500$ BEND_GPS $237.965 \quad 37.426 \quad-21.564 \quad 23.309 \quad 1.000 \quad 1.000$ CLIF_GPS $237.948 \quad 36.950 \quad-29.576 \quad 33.914 \quad 0.500 \quad 0.500$ CRO3_GPS 237.948 36.993 $-26.366 \quad 33.914 \quad 1.200 \quad 1.200$ SCAZ_GPS $237.944 \quad 36.978 \quad-29.770 \quad 33.115 \quad 1.200 \quad 1.200$ LOMP_GPS $237.941 \quad 37.099 \quad-23.643 \quad 33.716 \quad 1.100 \quad 1.200$ ROC2 2 GPS $237.939 \quad 37.815 \quad-15.581 \quad 15.317 \quad 0.500 \quad 0.500$ CAS2_GPS $237.931 \quad 37.732 \quad-16.301 \quad 16.620 \quad 0.600 \quad 0.600$ RDHL_GPS 237.905 37.551 $-21.046 \quad 22.627 \quad 0.500 \quad 0.600$ COYS_GPS $237.903 \quad 37.563-21.043 \quad 22.627 \quad 0.500 \quad 0.600$ $\begin{array}{lllllll}\text { VAC3 } 3 P S & 237.897 & 38.398 & -11.357 & 8.329 & 0.500 & 0.500\end{array}$ GORR_GPS $237.885 \quad 38.331 \quad-11.974 \quad 9.233 \quad 0.700 \quad 0.700$ FTHL_GPS $237.877 \quad 37.363-22.193 \quad 26.236 \quad 0.800 \quad 0.900$ HILL_GPS $237.872 \quad 37.937 \quad-14.064 \quad 15.837 \quad 0.600 \quad 0.600$ BM1R_GPS $237.847 \quad 37.290 \quad-23.714 \quad 29.444 \quad 0.700 \quad 0.700$ PAWT_GPS $237.833 \quad 37.324 \quad-23.908 \quad 29.148 \quad 0.600 \quad 0.600$ GAME_GPS $237.825 \quad 38.351 \quad-12.679 \quad 10.951 \quad 0.500 \quad 0.500$ SLBB_GPS $237.816 \quad 37.691 \quad-18.928 \quad 22.753 \quad 0.700 \quad 0.700$ EAUN_GPS $237.805 \quad 37.147 \quad-28.153 \quad 33.856 \quad 0.500 \quad 0.500$ MADI_GPS $237.797 \quad 38.313 \quad-13.791 \quad 13.959 \quad 0.500 \quad 0.500$ TRUE_GPS 237.784 $37.312 \quad-24.918 \quad 30.963 \quad 0.600 \quad 0.700$ BAPK_GPS 237.778 $37.884 \quad-16.190 \quad 18.065 \quad 0.500 \quad 0.600$ CROC GPS 237.771 $38.043 \quad-15.755 \quad 16.167 \quad 1.100 \quad 1.100$ 04GG GPS $237.753 \quad 37.879 \quad-16.595 \quad 17.272 \quad 0.800 \quad 0.800$ STAD_GPS $237.749 \quad 37.870 \quad-18.198 \quad 22.873 \quad 0.900 \quad 0.900$ STAC_GPS $237.748 \quad 37.871 \quad-17.898 \quad 22.273 \quad 0.900 \quad 0.900$ 0410_GPS $237.74538 .030-16.563 \quad 17.574 \quad 1.000 \quad 1.200$ 
HAGG_GPS $237.741 \quad 38.324 \quad-13.097 \quad 15.075 \quad 0.500 \quad 0.500$

UCBK_GPS $237.735 \quad 37.872 \quad-19.300 \quad 22.777 \quad 0.500 \quad 0.600$

FLES_GPS $237.726 \quad 37.900 \quad-18.394 \quad 18.980 \quad 1.900 \quad 1.300$

HAUL_GPS $237.716 \quad 37.274 \quad-24.937 \quad 33.883 \quad 0.900 \quad 1.000$

MA_A_GPS $237.695 \quad 37.937 \quad-19.691 \quad 22.989 \quad 1.000 \quad 0.800$

DEAL_GPS $237.662 \quad 38.258 \quad-14.325 \quad 16.699 \quad 0.700 \quad 0.800$

RMD1_GPS $237.660 \quad 37.934 \quad-19.398 \quad 23.100 \quad 1.400 \quad 1.700$

SPA1_GPS $237.644 \quad 37.990 \quad-15.887 \quad 18.804 \quad 0.900 \quad 0.900$

HALM_GPS $237.641 \quad 37.228 \quad-29.659 \quad 34.405 \quad 0.600 \quad 0.700$

HENN_GPS $237.638 \quad 38.283 \quad-14.022 \quad 18.606 \quad 1.700 \quad 1.900$

NAVY_GPS $237.634 \quad 37.810 \quad-20.629 \quad 25.007 \quad 0.500 \quad 0.600$

PEEE_GPS $237.618 \quad 37.592 \quad-21.880 \quad 28.812 \quad 0.700 \quad 0.700$

PIGE_GPS $237.605 \quad 37.183 \quad-28.575 \quad 35.616 \quad 0.600 \quad 0.600$

HIL4_GPS 237.598 $37.942 \quad-20.405 \quad 24.417 \quad 1.100 \quad 1.100$

$\begin{array}{llllllll}\text { SNPR_GPS } 237.584 & 37.959 & -20.103 & 23.022 & 0.800 & 0.900\end{array}$

PRSD_GPS 237.545 $37.805 \quad-22.544 \quad 26.333 \quad 0.800 \quad 0.800$

AIRR_GPS 237.544 38.223 $-16.351 \quad 18.833 \quad 0.500 \quad 0.500$

PALO_GPS 237.544 37.527 $\quad-26.207 \quad 31.833 \quad 0.600 \quad 0.600$

SWEE_GPS $237.542 \quad 37.609 \quad-24.888 \quad 30.834 \quad 0.600 \quad 0.600$

WHAL_GPS $237.496 \quad 37.509 \quad-26.518 \quad 33.248 \quad 0.700 \quad 0.700$

ADOO_GPS 237.473 38.236 $-17.059 \quad 21.855 \quad 0.500 \quad 0.500$

$\begin{array}{lllllllll}\text { COR_GPS } & 237.405 & 38.186 & -19.181 & 23.675 & 0.500 & 0.500\end{array}$

T3R2_GPS 237.401 37.923 $-22.740 \quad 26.376 \quad 0.600 \quad 0.600$

NICC_GPS $237.263 \quad 38.093 \quad-21.224 \quad 28.116 \quad 0.500 \quad 0.500$

1395_GPS $237.187 \quad 38.087 \quad-23.837 \quad 29.339 \quad 0.600 \quad 0.600$

PRH2_GPS $237.131 \quad 38.080 \quad-24.647 \quad 32.256 \quad 0.500 \quad 0.500$

PRNC_GPS $237.063 \quad 38.104 \quad-28.053 \quad 33.576 \quad 1.600 \quad 1.500$

THTI_GPS $210.394-17.577 \quad-66.958 \quad 47.557 \quad 0.700 \quad 0.600$ 